\title{
Minimização de funções decomponíveis em curvas em U definidas sobre cadeias de posets - algoritmos e aplicações
}

Marcelo da Silva Reis

TESE APRESENTADA

$\mathrm{AO}$

Instituto de Matemática e Estatística

DA

Universidade DE SÃo PAUlo

PARA

OBTENÇÃO DO TÍTULO

$\mathrm{DE}$

DOUTOR EM CIÊNCIAS

Programa: Ciência da Computação

Orientador: Prof. Dr. Junior Barrera

Durante o desenvolvimento deste trabalho o autor recebeu auxílio financeiro do CNPq

São Paulo, janeiro de 2013 


\section{Minimização de funções decomponíveis em curvas em U definidas sobre cadeias de posets - algoritmos e aplicações}

Este exemplar corresponde à redação final da tese devidamente corrigida e defendida por Marcelo da Silva Reis e aprovada pela Comissão Julgadora.

Esta versão definitiva da tese contém correções e alterações sugeridas pela Comissão Julgadora durante a defesa realizada por Marcelo da Silva Reis em 28 de novembro de 2012.

Comissão Julgadora:

- Prof.. Dr. Junior Barrera (orientador) - IME-USP

- Prof. Dr. Carlos Eduardo Ferreira - IME-USP

- Prof. Dr. Alair Pereira do Lago - IME-USP

- Prof. Dr. David Corrêa Martins Junior - UFABC

- Prof. Dr. Horacio Hideki Yanasse - INPE 
À Patrícia e aos meus pais 


\section{Agradecimentos}

Ao professor Junior Barrera pela oportunidade de me orientar nesta tese. Agradeço pela confiança em meu trabalho, pelo apoio nos momentos difíceis, e também pelas conversas e pela amizade ao longo dos últimos anos.

Ao professor Carlos Eduardo Ferreira, pela intensa colaboração neste trabalho e pelas várias conversas que tivemos, estas últimas frequentemente acompanhadas de valiosas dicas, tanto acadêmicas quanto profissionais.

A todos os professores do IME-USP que ajudaram em minha formação acadêmica, por conseguinte colaborando com este trabalho.

Aos amigos do Laboratório de Visão Computacional do IME-USP, em particular à Jihan, pela ajuda na revisão desta tese, e ao David Pires, pelo auxílio em alguns problemas burocráticos. Meus agradecimentos ao David Martins-Jr, ao Leandro Lima, ao Walter e a todos os demais colegas. Agradeço também aos amigos de outros laboratórios do IME, em particular ao Wesley e ao Tales.

Ao $\mathrm{CNPq}$ pelo financiamento da maior parte deste trabalho. Agradeço também ao professor Fabio Kon, que, ao me conceder uma bolsa parcial em um projeto do Laboratório de Computação Paralela e Distribuída do IME-USP, viabilizou a finalização deste trabalho.

Aos membros da Comissão Julgadora, pelas sugestões e críticas que deram uma grande contribuição para a elaboração da versão final desta tese.

A toda a minha família: aos meus irmãos, André e Adriana, ao Pepe, à dona Maria, ao seu Nino e à Marcia. Em especial, agradeço ao meu pai, seu Vavá, à minha mãe, dona Leide, e a minha companheira de jornada, Patrícia, pelo apoio irrestrito dado pelos mesmos nesta caminhada. Sem eles, certamente eu não conseguiria terminar esta empreitada.

Finalmente, agradeço a todos que contribuíram, direta ou indiretamente, para a realização deste trabalho. A todos, muito obrigado! 


\section{Resumo}

Título: Minimização de funções decomponíveis em curvas em U definidas sobre cadeias de posets algoritmos e aplicações

O problema de seleção de características, no contexto de Reconhecimento de Padrões, consiste na escolha de um subconjunto $X$ de um conjunto $S$ de características, de tal forma que $X$ seja "ótimo" dentro de algum critério. Supondo a escolha de uma função custo $c$ apropriada, o problema de seleção de características é reduzido a um problema de busca que utiliza $c$ para avaliar os subconjuntos de $S$ e assim detectar um subconjunto de características ótimo. Todavia, o problema de seleção de características é NP-difícil. Na literatura existem diversos algoritmos e heurísticas propostos para abordar este problema; porém, quase nenhuma dessas técnicas explora o fato que existem funções custo cujos valores são estimados a partir de uma amostra e que descrevem uma "curva em U" nas cadeias do reticulado Booleano $(\mathcal{P}(S), \subseteq$ ), um fenômeno bem conhecido em Reconhecimento de Padrões: conforme aumenta-se o número de características consideradas, há uma queda no custo do subconjunto avaliado, até o ponto em que a limitação no número de amostras faz com que seguir adicionando características passe a aumentar o custo, devido ao aumento no erro de estimação. Em 2010, Ris e colegas propuseram um novo algoritmo para resolver esse caso particular do problema de seleção de características, que aproveita o fato de que o espaço de busca pode ser organizado como um reticulado Booleano, assim como a estrutura de curvas em U das cadeias do reticulado, para encontrar um subconjunto ótimo.

Neste trabalho estudamos a estrutura do problema de minimização de funções custo cujas cadeias são decomponíveis em curvas em U (problema U-curve), provando que o mesmo é NP-difícil. Mostramos que o algoritmo de Ris e colegas possui um erro que o torna de fato sub-ótimo, e propusemos uma versão corrigida e melhorada do mesmo, o algoritmo U-CURVE-SEARCH (UCS). Apresentamos também duas variações do algoritmo UCS que controlam o espaço de busca de forma mais sistemática. Introduzimos dois novos algoritmos branch-and-bound para abordar o problema, chamados U-Curve-Branch-And-Bound (UBB) e Poset-Forest-Search (PFS). Para todos os algoritmos apresentados nesta tese, fornecemos análise de complexidade de tempo e, para alguns deles, também prova de corretude. Implementamos todos os algoritmos apresentados utilizando o arcabouço featsel, também desenvolvido neste trabalho; realizamos experimentos ótimos e sub-ótimos com instâncias de dados reais e simulados e analisamos os resultados obtidos. Por fim, propusemos um relaxamento do problema U-curve que modela alguns tipos de projeto de classificadores; também provamos que os algoritmos UCS, UBB e PFS resolvem esta versão generalizada do problema.

Palavras-chave: U-curve, busca ótima, seleção de características, branch-and-bound 


\section{Abstract}

Title: Minimization of decomposable in U-shaped curves functions defined on poset chains - algorithms and applications

The feature selection problem, in the context of Pattern Recognition, consists in the choice of a subset $X$ of a set $S$ of features, such that $X$ is "optimal" under some criterion. If we assume the choice of a proper cost function $c$, then the feature selection problem is reduced to a search problem, which uses $c$ to evaluate the subsets of $S$, therefore finding an optimal feature subset. However, the feature selection problem is NP-hard. Although there are a myriad of algorithms and heuristics to tackle this problem in the literature, almost none of those techniques explores the fact that there are cost functions whose values are estimated from a sample and describe a "U-shaped curve" in the chains of the Boolean lattice o $(\mathcal{P}(S), \subseteq)$, a well-known phenomenon in Pattern Recognition: for a fixed number of samples, the increase in the number of considered features may have two consequences: if the available sample is enough to a good estimation, then it should occur a reduction of the estimation error, otherwise, the lack of data induces an increase of the estimation error. In 2010, Ris et al. proposed a new algorithm to solve this particular case of the feature selection problem: their algorithm takes into account the fact that the search space may be organized as a Boolean lattice, as well as that the chains of this lattice describe a U-shaped curve, to find an optimal feature subset.

In this work, we studied the structure of the minimization problem of cost functions whose chains are decomposable in U-shaped curves (the U-curve problem), and proved that this problem is actually NP-hard. We showed that the algorithm introduced by Ris et al. has an error that leads to suboptimal solutions, and proposed a corrected and improved version, the U-CURVE-SEARCH (UCS) algorithm. Moreover, to manage the search space in a more systematic way, we also presented two modifications of the UCS algorithm. We introduced two new branch-and-bound algorithms to tackle the U-curve problem, namely U-CuRvE-BRANCH-AND-BOUnD (UBB) and Poset-Forest-Search (PFS). For each algorithm presented in this thesis, we provided time complexity analysis and, for some of them, also proof of correctness. We implemented each algorithm through the featsel framework, which was also developed in this work; we performed optimal and suboptimal experiments with instances from real and simulated data, and analyzed the results. Finally, we proposed a generalization of the U-curve problem that models some kinds of classifier design; we proved the correctness of the UCS, UBB, and PFS algorithms for this generalized version of the U-curve problem.

Keywords: U-curve, optimal search, feature selection, branch-and-bound 


\section{Sumário}

Lista de Abreviaturas $\quad$ xiii

Lista de Símbolos $\quad$ Xv

Lista de Figuras $\quad$ xvii

Lista de Tabelas $\quad$ xxiii

1 Introdução $\quad 1$

1.1 Objetivos deste trabalho . . . . . . . . . . . . . . . . 2

1.2 Organização da tese . . . . . . . . . . . . . . . . . . . . . . 3

1.2.1 Sequências possíveis de leitura . . . . . . . . . . . . . . . 4

2 Revisão bibliográfica e conceitos fundamentais $\quad 5$

2.1 O problema de seleção de características . . . . . . . . . . . . . . . . . . 5

2.1.1 Funções custo de seleção de características . . . . . . . . . . . . . . . . . 6

2.1.2 Algoritmos de seleção de características . . . . . . . . . . . . . . . . . . 6

2.1.3 Um caso particular: o problema U-curve . . . . . . . . . . . . . . . . . . 8

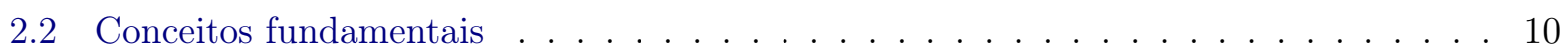

2.2 .1 Definições iniciais . . . . . . . . . . . . . . . . . . . . . 10

2.2 .2 Estrutura dos experimentos realizados . . . . . . . . . . . . . . . . 12

3 O problema U-curve $\quad 15$

3.1 Formalização do problema U-curve . . . . . . . . . . . . . . . . . . . 15

3.2 Análise de complexidade do problema U-curve . . . . . . . . . . . . . . . . . . 15

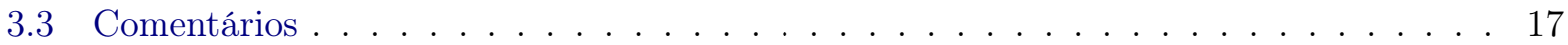

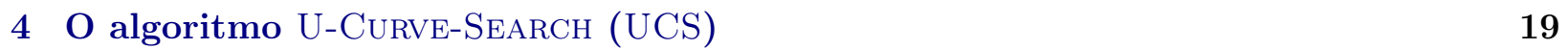

4.1 Problema com o algoritmo U-CuRve . . . . . . . . . . . . . . . . . . . . . 19

4.2 Princípios de funcionamento do novo algoritmo . . . . . . . . . . . . . . . 20

4.3 Pseudo-código e complexidade de tempo . . . . . . . . . . . . . . . . . . . 29

4.3.1 Atualização das restrições inferiores e superiores . . . . . . . . . . . . . . . 29

4.3.2 Elementos minimais e maximais . . . . . . . . . . . . . . . . . . 30

4.3 .3 Busca em profundidade . . . . . . . . . . . . . . . . . 30

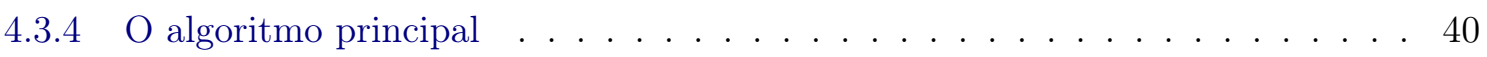

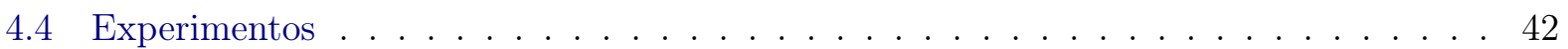


4.4 .1 Análise dos experimentos . . . . . . . . . . . . . . . . . . . . 42

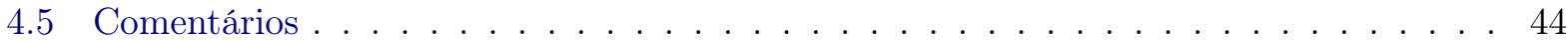

$5 \quad$ O problema da busca de início de caminho $\quad 47$

5.1 Formalização do problema e análise de complexidade . . . . . . . . . . . . . . 47

5.1 .1 Busca de início de caminho no algoritmo UCS . . . . . . . . . . . . 50

5.2 Abordagem do problema usando Programação Linear Inteira . . . . . . . . . . . . . 50

5.2 .1 Experimentos . . . . . . . . . . . . . . . . . 51

5.3 Abordagem do problema usando uma nova estrutura de dados . . . . . . . . . . . 52

5.3.1 Estrutura de dados para armazenamento de coleção de restrições . . . . . . . . 53

5.3 .2 Algoritmo de busca de início de caminho . . . . . . . . . . . . . . . 56

5.3 .3 Experimentos . . . . . . . . . . . . . . . . . . . . . 60

5.4 Discussão . . . . . . . . . . . . . . . . . . . . . . . . . 60

5.4 .1 Múltiplas buscas em profundidade . . . . . . . . . . . . . 61

5.4 .2 Operações de interseções de conjuntos . . . . . . . . . . . . . . . . . 62

5.4 .3 Aninhamento de coleções de restrições e grafo tripartido . . . . . . . . . . . . 64

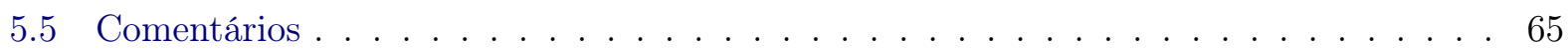

6 Algoritmos branch-and-bound para o problema U-curve $\quad 67$

6.1 O algoritmo U-Curve-Branch-And-Bound (UBB) . . . . . . . . . . . . . 67

6.1 .1 Limitações do algoritmo UBB . . . . . . . . . . . . . . . . . 74

6.2 O algoritmo Poset-Forest-SeArch $(\mathrm{PFS}) \ldots \ldots \ldots \ldots \ldots$

6.2.1 Princípios de funcionamento do novo algoritmo . . . . . . . . . . . . 75

6.2 .2 Pseudo-código e complexidade de tempo . . . . . . . . . . . . . . . 87

6.3 Experimentos . . . . . . . . . . . . . . . . . . . . . 93

6.3.1 Análise dos experimentos . . . . . . . . . . . . . . . . . 93

6.4 Comentários . . . . . . . . . . . . . . . . . . . . . . . 93

$\begin{array}{llr}7 & \text { Experimentos } & 97\end{array}$

7.1 Instâncias empregadas . . . . . . . . . . . . . . . . . . . . . . 97

7.1 .1 Simulação de instâncias difíceis . . . . . . . . . . . . . . . . . . 97

7.1.2 Projeto de W-operadores . . . . . . . . . . . . . . . . . . . 97

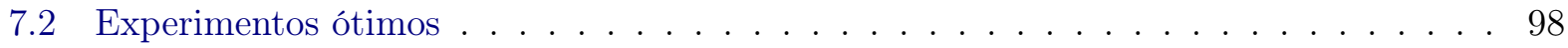

7.2.1 Simulação de instâncias difíceis . . . . . . . . . . . . . . . . . . 98

7.2 .2 Projeto de W-operadores . . . . . . . . . . . . . . . . . . . . . . 98

7.3 Experimentos sub-ótimos f . . . . . . . . . . . . . . . . . . 101

7.3 .1 Simulação de instâncias difíceis . . . . . . . . . . . . . . . . . . . . . . . . . . . . . . . . . . . . . . . . . .

7.3 .2 Projeto de W-operadores . . . . . . . . . . . . . . . . . . . 102

7.4 Análise dos resultados . . . . . . . . . . . . . . . . . . . 102

7.4.1 Análise de desempenho dos algoritmos . . . . . . . . . . . . . . . . . . 102

7.4.2 Análise da complexidade de tempo computacional . . . . . . . . . . . 106 
8.1 Funções parcialmente decomponíveis em curvas em U . . . . . . . . . . . . . . . 113

8.2 Aplicação no projeto de classificadores . . . . . . . . . . . . . . . . . . . 114

8.2.1 Um exemplo de aplicação . . . . . . . . . . . . . . . . . . . . . . 117

8.3 Corretude dos algoritmos UCS, UBB e PFS . . . . . . . . . . . . . . . 117

8.4 Comentários . . . . . . . . . . . . . . . . . . . . . . . . . . 119

9 Conclusões $\quad 121$

9.1 Revisão e discussão dos resultados apresentados . . . . . . . . . . . . . . . . . 121

9.2 Contribuições deste trabalho . . . . . . . . . . . . . . . . . . . . . 123

9.2 .1 Contribuições científicas . . . . . . . . . . . . . . . . . . . . . 123

9.2 .2 Contribuições tecnológicas . . . . . . . . . . . . . . . . . . . . . . . 124

9.3 Lista de publicações . . . . . . . . . . . . . . . . . . . . . . . . . . . . 124

9.4 Possibilidades de trabalhos futuros . . . . . . . . . . . . . . . . 125

$\begin{array}{ll}\text { A O arcabouço featsel } & \mathbf{1 2 7}\end{array}$

A.1 Funções custo implementadas . . . . . . . . . . . . . . . . . . . . . 127

A.2 Algoritmos implementados . . . . . . . . . . . . . . . . . . . . 129

$\begin{array}{ll}\text { B O algoritmo Exhaustive-Search (ES) } & 131\end{array}$

C Implementação de função custo que utiliza entropia condicional média 133

Referências Bibliográficas 135

$\begin{array}{ll}\text { Índice Remissivo } & 139\end{array}$ 


\section{Lista de Abreviaturas}

ASFFS Busca Sequencial Flutuante para Frente Adaptativa (Adaptative Sequential Forward Floating Search)

BFS Busca em Largura (Breadth-First Search)

DFS Busca em Profundidade (Depth-First Search)

ED Estrutura de Dados

ES Busca Exaustiva (Exhaustive Search)

hSFFS Busca Sequencial Flutuante para Frente Híbrida e Flexível (Flexible-hybrid Sequential Forward Floating Search)

ISFFS Busca Sequencial Flutuante para Frente Melhorada (Improved Sequential Forward Floating Search)

MAE Erro Absoluto Médio (Mean Absolute Error)

PFS Busca de Floresta de Posets (Poset Forest Search)

PGN Rede Gênica Probabilística (Probabilistic Gene Network)

PL Programação Linear

PLI Programação Linear Inteira

SBS Busca Sequencial para Trás (Sequential Backward Search)

SBFS Busca Sequencial Flutuante para Trás (Sequential Backward Floating Search)

SFFS Busca Sequencial Flutuante para Frente (Sequential Forward Floating Search)

SFS Busca Sequencial para Frente (Sequential Forward Search)

UBB U-Curve Branch-and-Bound

UCS Busca U-Curve (U-Curve Search)

UML Linguagem de Modelagem Unificada (Unified Modeling Language)

XML Linguagem de Marcação Estendida (Extended Markup Language) 


\title{
Lista de Símbolos
}

\author{
$\chi^{S} \quad$ vetor característico do conjunto $S$ \\ $|S| \quad$ cardinalidade (i.e., número de elementos) do conjunto $S$ \\ $\mathcal{K}_{\psi} \quad$ núcleo (kernel) do classificador $\psi$ \\ $\mathcal{P}(S) \quad$ conjunto potência do conjunto $S$ \\ $(\mathcal{P}(S), \subseteq) \quad$ reticulado Booleano de grau $|S|$ \\ $\mathbb{R}+\quad$ conjunto dos números reais não-negativos \\ $\mathcal{R}_{L} \quad$ coleção de restrições inferiores \\ $\mathcal{R}_{U} \quad$ coleção de restrições superiores \\ $\mathcal{X}\left(\mathcal{R}_{L}, \mathcal{R}_{U}\right)$ espaço de busca corrente definido por $\mathcal{R}_{L}$ e $\mathcal{R}_{U}$ \\ $\psi \quad$ classificador \\ $\Psi \quad$ espaço dos classificadores
}




\section{Lista de Figuras}

1.1 um exemplo de instância do problema U-curve. Figura 1.1(a): o diagrama de Hasse de um reticulado Booleano de grau 3 - as cadeias do reticulado, cujos custos de seus elementos são definidos através dos números ao lado dos nós, descrevem curvas em U; a cadeia maximal $\{\emptyset, c, b c, a b c\}$ está destacada em negrito. O elemento $b c$, destacado em verde, é mínimo na cadeia (e também no reticulado Booleano). Figura 1.1(b): o gráfico dos custos em função dos elementos da cadeia maximal destacada em negrito, mostrando sua curva em U. . . . . . . . . . . . . . . . . . . 2

1.2 fluxograma que ilustra sequências possíveis de leitura desta tese. . . . . . . . . . . 4

2.1 a taxonomia de alguns algoritmos de seleção de características utilizados em Reconhecimento de Padrões. Adaptado de Jain e colegas, 1997 [JZ97]. . . . . . . . . . . . 8

2.2 a localização do algoritmo U-CuRve na taxonomia de algoritmos de seleção de características exibida na figura 2.1 - U-CuRve é um algoritmo que foi projetado com a intenção de ser ótimo e estocástico. . . . . . . . . . . . . . . . . . . . 9

2.3 os caminhos percorridos em um reticulado Booleano por diferentes algoritmos de busca. "Busca bidirecional" consiste na execução dos algoritmos SBS e SFS, na qual toma-se uma melhor solução obtida por ambos. Adaptado de Siedlecki e Sklansky

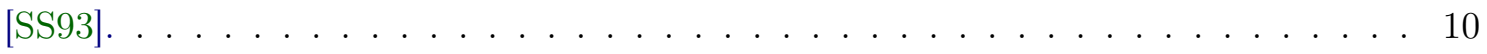

2.4 um grafo $G$ com cinco vértices e sete arestas. Os vértices em amarelo constituem uma cobertura de vértices de $G$; tal cobertura é mínima. . . . . . . . . . . . . . 11

4.1 um contra-exemplo da correção da sub-rotina Minimum-Exhausted do algoritmo U-Curve. . . . . . . . . . . . . . . . . . . . . . . 20

4.2 continuação do contra-exemplo da figura 4.1, que mostra uma simulação da subrotina Minimum-Exhausted que tem uma sequência de remoção de elementos que leva a perda de 00001, o único mínimo global do espaço de busca (figura 4.2(b)). . . 21

4.3 uma simulação do novo algoritmo para abordar o problema U-curve. . . . . . . . . . 22

4.4 continuação da simulação iniciada na figura $4.3 \ldots \ldots$. . . . . . . . . . . . 23

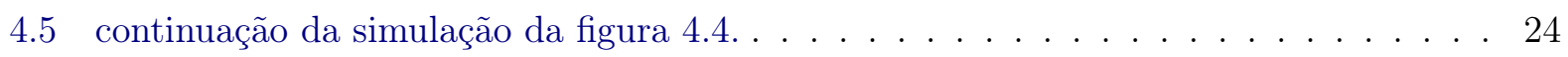

4.6 continuação da simulação da figura 4.5 . . . . . . . . . . . . . . . . . 25

4.7 continuação da simulação da figura $4.6 \ldots \ldots \ldots \ldots \ldots$

4.8 continuação da simulação da figura $4.7 \ldots \ldots \ldots \ldots \ldots \ldots$. . . . . . . . . . . 27

4.9 continuação da simulação da figura $4.8 \ldots \ldots \ldots \ldots \ldots$ 
4.10 uma outra simulação de UCS, que mostra uma situação em que não bastam as arestas do sub-grafo para realizar uma poda. Neste caso, é necessário fazer a poda em duas etapas: primeiro, a remoção utiliza as arestas; segundo, são inspecionados todos os vértices do sub-grafo obtido da primeira etapa. . . . . . . . . . . . . . . . 32

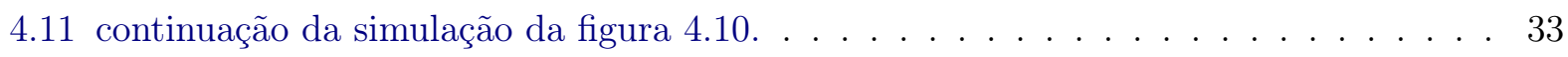

4.12 continuação da simulação da figura $4.11 \ldots \ldots$. . . . . . . . . . . . . . 34

4.13 continuação da simulação da figura 4.12 . . . . . . . . . . . . . . . . 35

4.14 a evolução do tamanho máximo de um grafo $G$ em busca ótima (figura 4.14(a)) e sub-ótima (figura 4.14(b)), durante a execução do algoritmo UCS em instâncias simuladas. Cada linha mostra, para diferentes tamanhos de $S$, a razão entre $2^{|S|}$ (i.e., o tamanho do espaço de busca) e o tamanho máximo do grafo. As linhas vermelha e azul foram desenhadas utilizando os mesmos dados empregados para produzir as tabelas 4.1 e 4.3 , respectivamente. . . . . . . . . . . . . . . .

4.15 comparação entre o número de vezes que a sub-rotina DFS é chamada (linha sólida) e o número de iterações do algoritmo principal (linha tracejada), durante a execução do algoritmo UCS em instâncias de diferentes tamanhos. Tanto na figura 4.15(a) (experimentos ótimos) quanto na figura 4.15(b) (experimentos sub-ótimos) observamos que para instâncias de tamanho 18 o número de iterações do algoritmo principal é ao menos duas ordens de magnitude maior do que o número de chamadas de DFS.

5.1 exemplo de construção da redução polinomial utilizando como instância do problema da cobertura de vértices o grafo $G$ da figura 2.4. Existe uma cobertura de vértices de $G$ com tamanho até $k^{\prime}$ se e somente se existe um elemento $X$ em $\mathcal{X}\left(\mathcal{R}_{L}, \mathcal{R}_{U}\right)$, tal

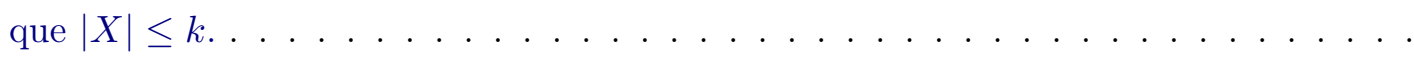

5.2 um exemplo de aplicação da nova ED para uma instância de 5 elementos. A figura 5.2(a) mostra um espaço de busca corrente $\mathcal{X}\left(\mathcal{R}_{L}, \emptyset\right)$ definido pela coleção de restrições inferiores $\mathcal{R}_{L}=\{11000,01110,00111,01011\}$, enquanto que a figura 5.2(b) ilustra a nova ED armazenando estas quatro restrições; observe que a ordenação estável foi aplicada na ED, e que portanto a coleção de elementos da primeira linha tem cardinalidade máxima.

5.3 um contra-exemplo de que a escolha gulosa das linhas da ED com as maiores coleções de elementos de $\mathcal{R}_{L}$ não serve para resolver o problema da busca de início de caminho. Escolhendo as duas primeiras linhas (figura 5.3(a)), temos que $X=10100$ não pertence a $\mathcal{X}\left(\mathcal{R}_{L}, \mathcal{R}_{U}\right)$ (figura $\left.5.3(\mathrm{~b})\right) \ldots \ldots \ldots \ldots \ldots$

5.4 árvore de busca $T$ produzida através de uma enumeração dos elementos do exemplo da figura 5.2(b). $T$ foi construída através de aplicações recursivas da decomposição do espaço de busca que é apresentada no lema 6.1.1. . . . . . . . . . . . . . 56

5.5 um exemplo de percorrimento de uma árvore de busca $T$, visando encontrar um início de caminho. Figura 5.5(a): um percorrimento de $T$ até encontrar o elemento $a b d=11010$; este elemento cobre todos as restrições na ED da figura 5.2(b), portanto ele não é coberto por $\mathcal{R}_{L}$. Figura 5.5(b): verificamos que 11010 pertence ao espaço de busca corrente, pois este elemento não é coberto por $\mathcal{R}_{U} \ldots \ldots \ldots$. . . . . . . 
5.6 comparação entre o número total de vezes que a sub-rotina DFS é chamada (linha tracejada) com o número de vezes em que a chamada dessa sub-rotina visita apenas um elemento (linha sólida), durante a execução do algoritmo UCS-3 no experimento mostrado na tabela 5.3. Observe que para instâncias de tamanho 16 cerca de metade das chamadas dessa sub-rotina visitam um único elemento. . . . . . . . . . . . . . . . 62

5.7 um exemplo de grafo tripartido para representar os 4 elementos de um conjunto $S$, duas coleções de restrições (no caso, $\mathcal{R}_{L}=\{1100,1001\}$ e $\mathcal{R}_{U}=\{1000,0001\}$ ) que são aninhadas, as interseções entre os elementos de uma mesma coleção e também entre os elementos de coleções distintas. . . . . . . . . . . . . . . . . . . .

6.1 uma simulação de U-CuRve-BRANCH-AND-BOUnd, um algoritmo branch-and-bound para abordar o problema U-curve. . . . . . . . . . . . . . . . . . . . 69

6.2 continuação da simulação da figura $6.1 \ldots \ldots \ldots \ldots \ldots \ldots$

6.3 continuação da simulação da figura $6.2 \ldots \ldots \ldots \ldots$. . . . . . . . . . . 71

6.4 continuação da simulação da figura $6.3 \ldots \ldots \ldots$. . . . . . . . . . . . . . 72

6.5 um exemplo de instância que exige que o algoritmo UBB visite todos os elementos do espaço de busca. Figura 6.5(a): o reticulado Booleano definido pelos elementos da instância; Figura 6.5(b): uma enumeração do reticulado Booleano que define uma árvore $T$; observe que todo elemento de custo mínimo nas cadeias definidas por caminhos em $T$ são folhas ou adjacentes inferiores a folhas, o que obriga o algoritmo a visitar todos os vértices de $T \ldots \ldots \ldots \ldots$. . . . . . . . . . . . . 75

6.6 um exemplo de enumerações de um reticulado Booleano, utilizando aplicações recursivas das decomposições dos lemas 6.1.1 (figura 6.6(a)) e 6.2.1 (figura 6.6(b)). Observe que para qualquer aresta $\{X, Y\}$ na árvore $T$ existe uma aresta $\left\{X^{c}, Y^{c}\right\}$ na árvore T'.

6.7 um exemplo de uma poda realizada na árvore $T$, no qual é eliminado do espaço de busca o intervalo [11100,11111] (figura 6.7(a)). A eliminação desse intervalo em $T^{\prime}$ produz uma floresta contendo três árvores (figura 6.7(b)). As raízes das árvores estão destacadas em amarelo. . . . . . . . . . . . . . . . 78

6.8 uma simulação de Poset-Forest-SeArch (PFS), um algoritmo branch-and-bound para abordar o problema U-curve. . . . . . . . . . . . . . . . 80

6.9 continuação da simulação da figura $6.8 \ldots \ldots \ldots \ldots$. . . . . . . . . . . 81

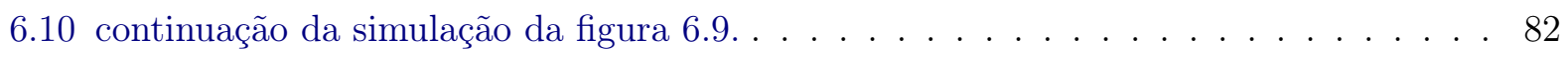

6.11 continuação da simulação da figura $6.10 \ldots \ldots$. . . . . . . . . . . . 83

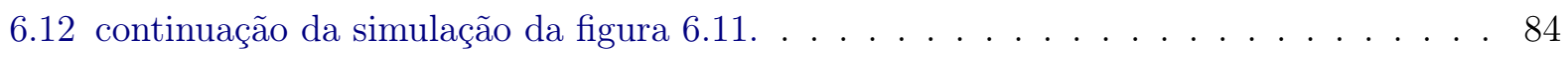

6.13 continuação da simulação da figura $6.12 \ldots \ldots \ldots \ldots \ldots \ldots$

6.14 continuação da simulação da figura $6.13 \ldots \ldots$. . . . . . . . . . . . 86

6.15 a evolução da soma dos tamanhos máximos das florestas $\mathcal{A}$ e $\mathcal{B}$ em uma busca ótima (figura 6.15(a)) e sub-ótima (figura 6.15(b)), durante execuções do algoritmo PFS em instâncias simuladas. Os gráficos sugerem que o tamanho das florestas tem um limite superior assintótico linear ao tamanho do espaço de busca. . . . . . . . . . . 96 
7.1 comparação entre UCS, UBB, PFS e SFFS, no experimento ótimo com instâncias difíceis simuladas. Na figura 7.1(a) é mostrada uma comparação de tempo médio que os algoritmos gastaram para resolver diversos tamanhos de instâncias; já na figura 7.1(b) é exibido o número médio de nós computados pelos algoritmos para os mesmos tamanhos de instâncias. . . . . . . . . . . . . . . . . . . . 100

7.2 comparação entre UCS, UBB, PFS e SFFS, no experimento ótimo de projeto de W-operador. Na figura 7.2(a) é mostrada uma comparação de tempo médio que os algoritmos gastaram para resolver cada teste; já na figura 7.2(b) é exibido o número médio de nós computados pelos algoritmos para os mesmos testes. . . . . . . . . . 100

7.3 comparação entre UCS, UBB, PFS e SFFS, no experimento sub-ótimo com instâncias difíceis simuladas. Na figura 7.3(a) é mostrada uma comparação de tempo médio que os algoritmos gastaram para resolver diversos tamanhos de instâncias; já na figura 7.3(b) é exibido o número médio de nós computados pelos algoritmos para os mesmos tamanhos de instâncias; por fim, na figura 7.3(c) é fornecido o número de vezes que cada algoritmo encontrou uma melhor solução, também para os mesmos tamanhos de instâncias. . . . . . . . . . . . . . . . . . . . . 103

7.4 comparação entre UCS, UBB, PFS e SFFS, no experimento sub-ótimo de projeto de W-operador. Na figura 7.4(a) é mostrada uma comparação de tempo médio que os algoritmos gastaram para resolver cada teste; já na figura 7.4(b) é exibido o número médio de nós computados pelos algoritmos para os mesmos testes. . . . . . . . . . 105

7.5 comparação entre os tempos computacionais (em log base 2 mili-segundos) requeridos por UCS (figura 7.5(a)), UBB (figura 7.5(b)) e PFS (figura 7.5(c)), nas instâncias difíceis mostradas na tabela 7.1. As barras vermelhas representam o tempo gasto em cálculo da função custo, enquanto que as barras verdes representam o tempo gasto nas ações remanescentes de cada algoritmo. O algoritmo UCS sempre exigiu mais tempo computacional em tarefas que não envolvem o cálculo da função custo; por outro lado, o fenômeno oposto ocorreu com os demais algoritmos. . . . . . . . . . . 105

7.6 comparação entre os tempos computacionais (em segundos) requeridos por UCS (figura 7.6(a)), UBB (figura 7.6(b)) e PFS (figura 7.6(c)), nos experimentos de projeto de W-operador mostrados na tabela 7.2. As barras vermelhas representam o tempo gasto em cálculo da função custo, enquanto que as barras verdes representam o tempo gasto nas ações remanescentes de cada algoritmo. . . . . . . . . . . . . . . . . 107

7.7 comparação entre os tempos computacionais (em segundos) requeridos por UCS (figura 7.7(a)) e UBB (figura 7.7(b)), no experimento de projeto de W-operador mostrados na tabela 7.7. Neste experimento, cada instância utilizou o dobro de amostras em relação aos experimentos utilizados para produzir os histogramas 7.6(a) e 7.6(b); observe que para vários testes UCS realizou a sua execução em menos tempo em relação ao algoritmo UBB. . . . . . . . . . . . . . . . . . . . . 107

8.1 um reticulado Booleano de grau 3, com uma função custo da distância de Hamming. Neste caso, $M=101$. Observe que $c$ é uma função custo parcialmente decomponível em curvas em U; todavia, $c$ não é uma função custo decomponível em curvas em U. . 114 8.2 o reticulado Booleano dos núcleos de todos os classificadores de $S=\{a, b\} \ldots$. . 118 
8.3 o reticulado Booleano dos núcleos de todos os classificadores de $S=\{a, b\}$, com os valores de uma função custo do erro absoluto médio que utiliza a distribuição conjunta especificada na tabela 8.2. $\mathcal{K}_{\psi_{7}}$ e $\mathcal{K}_{\psi_{8}}$ são os mínimos de $\mathcal{P}(\mathcal{P}(S)$ ); todas as cadeias maximais que contêm esses mínimos descrevem uma curva em $U$ e estão assinaladas com as arestas em negrito . . . . . . . . . . . . . . . . . 118

A.1 modelo de objetos em UML do arcabouço featsel. . . . . . . . . . . . . . . 128 


\section{Lista de Tabelas}

4.1 comparação entre UCS, SFFS e Exhaustive-SEARCH (ES), utilizando como função custo a equação 3.1 (i.e., uma redução polinomial de instâncias do problema da soma de subconjuntos, um problema NP-difícil). Apesar de que o tempo computacional de UCS aumenta mais rapidamente que o da busca exaustiva, o primeiro precisa computar menos vezes a função custo do que o segundo. . . . . . . . . . . . . . . 43

4.2 pré-processamento que produz os limiares para a busca subótima em instâncias difíceis. 43

4.3 busca subótima em instâncias difíceis; os limiares para cada teste foram obtidos através do pré-processamento exibido na tabela $4.2 \ldots \ldots \ldots$. . . . . . . . . 44

5.1 comparação entre UCS e UCS-2 (busca de início de caminho utilizando PL), com este último sempre buscando um início de caminho de cardinalidade mínima (máxima). Embora ambos os algoritmos sejam equivalentes do ponto de vista semântico, UCS mostrou-se mais eficiente do ponto de vista computacional. . . . . . . . . . . . . 52

5.2 comparação entre UCS e UCS-2 (busca de início de caminho utilizando PL), com este último buscando um início de caminho qualquer (i.e., devolvendo a primeira solução viável encontrado pelo branch and cut). Apesar de que ambos os algoritmos sejam equivalentes do ponto de vista semântico, UCS mostrou-se mais eficiente do ponto de vista computacional, embora a diferença entre eles seja menor do que a

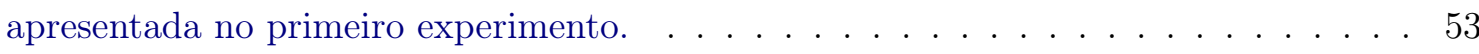

5.3 comparação entre UCS e UCS-3 (busca de início de caminho utilizando uma nova estrutura de dados e um novo algoritmo de busca). Ambos os algoritmos são equivalentes do ponto de vista semântico, mas UCS mostrou-se mais eficiente do ponto de vista computacional a partir de instâncias de tamanho $15 \ldots \ldots$. . . . . . . . 61

6.1 comparação ótima entre UBB, PFS, SFFS e Exhaustive-SEArch (ES), utilizando como função custo a equação 3.1 (i.e., uma redução polinomial de instâncias do problema da soma de subconjuntos, um problema NP-difícil). . . . . . . . . . . . 94

6.2 pré-processamento que produz os limiares para a busca sub-ótima em instâncias simuladas. . . . . . . . . . . . . . . . . . . . . . 95

6.3 busca sub-ótima em instâncias simuladas; os limiares para cada teste foram obtidos através do pré-processamento exibido na tabela $6.2 \ldots \ldots \ldots$. . . . . . . 95

7.1 comparação entre UCS, UBB, PFS e SFFS, utilizando como função custo a equação 3.1 (i.e., uma redução polinomial de instâncias do problema da soma de subconjuntos, um problema NP-difícil). . . . . . . . . . . . . . . . . . . 99 
7.2 comparação entre UCS, UBB, PFS e SFFS, no projeto de W-operador, utilizando como função custo a entropia condicional média penalizada (equação 7.2) . . . . . . . . 101

7.3 pré-processamento que produz os limiares para a busca sub-ótima em instâncias difíceis.102

7.4 busca sub-ótima em instâncias difíceis; os limiares para cada teste foram obtidos através do pré-processamento exibido na tabela 7.3 . . . . . . . . . . . . . . . . 104

7.5 pré-processamento que produz os limiares para a busca sub-ótima no projeto de W-operador. . . . . . . . . . . . . . . . . . . . . 106

7.6 busca sub-ótima no projeto de W-operador; os limiares para cada teste foram obtidos através do pré-processamento exibido na tabela 7.5 . . . . . . . . . . . . . . . 108

7.7 Comparação entre os algoritmos UCS e UBB, durante num experimento ótimo de projeto de $\mathrm{W}$-operadores utilizando as mesmas 14 instâncias de dados reais utilizadas para produzir a tabela 7.2, porém empregando o dobro de amostras (1152) em relação

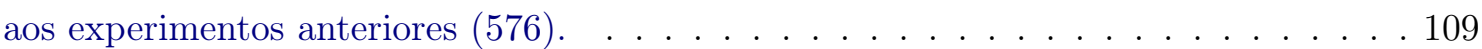

8.1 todos os possíveis classificadores de $S$; como $|S|=2$, temos que $|\Psi|$ (o tamanho do espaço de classificadores) é igual a $2^{2^{|S|}}=2^{2^{2}}=16 \ldots \ldots \ldots \ldots \ldots$. . . . . . . . . .

8.2 todos os valores da distribuição conjunta $P(\mathbf{y}, \mathbf{X})$, acompanhados dos valores de três classificadores de $S=\{a, b\}: \psi_{1}, \psi_{7}$ e $\psi_{13}$. O núcleo do classificador $\psi_{7}$ é mínimo em

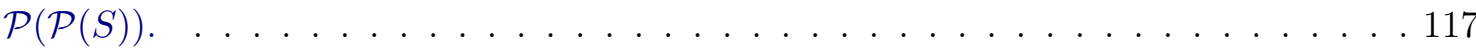




\section{Capítulo 1}

\section{Introdução}

O problema de seleção de características é um problema central em Reconhecimento de Padrões e, por essa razão, há muitos anos que o mesmo é estudado pela comunidade científica [DHS01, TK06]. Este problema pode ser entendido como um problema combinatório, que consiste em encontrar um subconjunto $X$ de um conjunto finito de características $S$, tal que $X$ minimize uma função custo $c$, definida a partir de $\mathcal{P}(S)$, o conjunto potência de $S$, para $\mathbb{R}+$. Se $X$ é um subconjunto de $S$ e $c(X)$ é mínimo (i.e., não existe um outro subconjunto $Y$ de $S$ tal que $c(Y)<c(X)$ ), então $X$ é um conjunto ótimo de características ou um conjunto de características de custo mínimo.

O modelo do problema de seleção de características é definido por uma função custo que tem impacto na complexidade do algoritmo de busca correspondente. Em geral, o problema é NP-difícil $\left[\mathrm{JKP}^{+}\right.$94]. Em modelos realísticos, a função custo depende da estimação de uma distribuição de probabilidade conjunta.

Uma vez definido o modelo do problema de seleção de características a ser resolvido, a próxima etapa é a escolha de um algoritmo de seleção de características apropriado. Na literatura existem diversos algoritmos e heurísticas propostos para resolver o problema de seleção de características; alguns deles exploram a técnica branch-and-bound [SPK04]. Uma das heurísticas bem sucedidas é SFFS, Sequential Forward Floating Selection [PNK94], que apresenta bons resultados consumindo pouco tempo computacional [RBMJ10]. Existem ainda técnicas que exploram o espaço de busca como um reticulado Booleano, percorrendo suas cadeias como em um grafo [SS93]. Entretanto, nenhuma dessas técnicas explora o fato que existem funções custo que dependem da estimação de uma distribuição de probabilidade conjunta e que descrevem, nas cadeias maximais do reticulado Booleano, uma "curva em U", um fenômeno bem conhecido em Reconhecimento de Padrões: conforme aumenta-se o número de características consideradas, a tendência é do custo cair, até o ponto em que a limitação no número de amostras faça com que seguir adicionando características passe a aumentar o custo, algo que ocorre devido ao aumento do erro de estimação [DHS01, JM00]. Dessa forma, é possível considerar um caso particular do problema de seleção de características que leve em consideração o fenômeno da "curva em U"; este caso particular também é conhecido como problema U-curve. Na figura 1.1(a) é mostrado um exemplo de uma instância do problema U-curve constituída de um reticulado Booleano de grau três, enquanto que na figura 1.1(b) é destacada uma cadeia deste reticulado.

Existem importantes aplicações práticas em Reconhecimento de Padrões cujas etapas de seleção de características podem ser entendidas como uma minimização de uma função custo cujas cadeias descrevem curvas em U: por exemplo, a identificação de preditores na estimação de Redes Gênicas Probabilísticas [BCJMJ+07] e o projeto de W-Operadores [MJCJB06]. Recentemente, foi proposto um novo algoritmo para resolver o problema U-curve: o algoritmo em questão, U-CURVE, homônimo ao problema, aproveita em sua busca a estrutura de curvas em $U$ das cadeias maximais, assim como o fato de que o espaço de busca pode ser organizado como um reticulado Booleano. $\mathrm{O}$ algoritmo U-CuRve demonstrou ter um desempenho satisfatório para diversas instâncias de interesse prático [Ris08, RBMJ10]. 


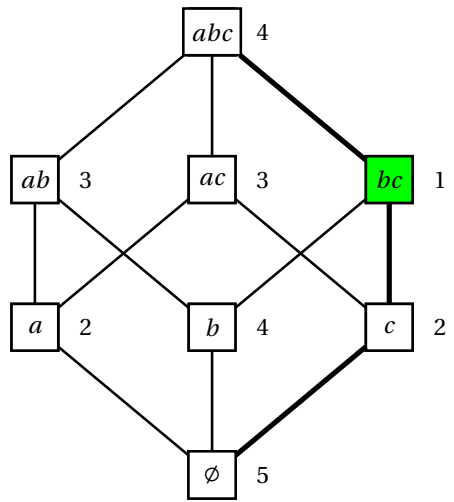

(a)

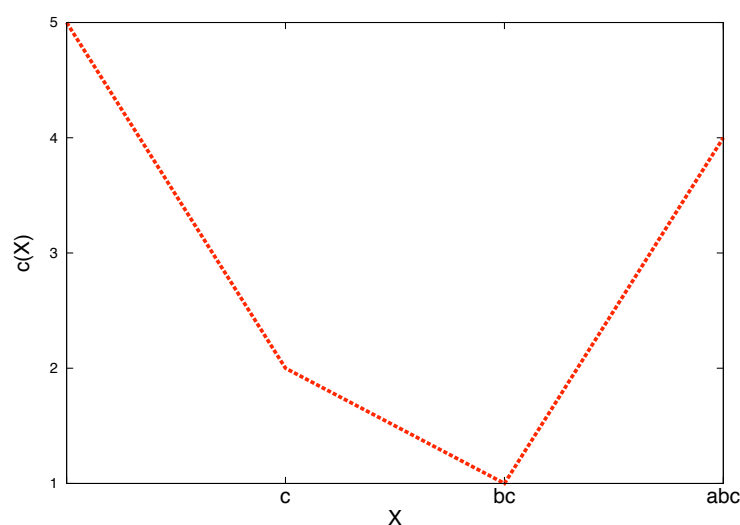

(b)

Figura 1.1: um exemplo de instância do problema U-curve. Figura 1.1(a): o diagrama de Hasse de um reticulado Booleano de grau 3 - as cadeias do reticulado, cujos custos de seus elementos são definidos através dos números ao lado dos nós, descrevem curvas em $U$; a cadeia maximal $\{\emptyset, c, b c$, abc $\}$ está destacada em negrito. O elemento bc, destacado em verde, é mínimo na cadeia (e também no reticulado Booleano). Figura 1.1(b): o gráfico dos custos em função dos elementos da cadeia maximal destacada em negrito, mostrando sua curva em $U$.

\subsection{Objetivos deste trabalho}

Esta tese teve como um dos objetivos principais estudar alguns aspectos da estrutura do problema U-curve, mais especificamente:

- a complexidade computacional do problema, algo que tem impacto direto no melhor desempenho possível por parte dos algoritmos utilizados para resolvê-lo;

- generalizações do problema U-curve que permitam modelar alguns problemas de interesse prático, como por exemplo o projeto de classificadores. Em particular, estudamos o caso em que nem todas as cadeias do reticulado Booleano descrevem uma curva em U.

A partir dos estudos de tais aspectos do problema U-curve, realizamos o outro objetivo central desta tese, que é o desenvolvimento de algoritmos ótimos para abordar o problema U-curve e a generalização do mesmo que foi descrita anteriormente. Este objetivo envolveu:

- melhoramentos no algoritmo U-CURVE apresentado por Ris e colegas;

- desenvolvimento de novos algoritmos ótimos para atacar o problema U-curve;

- avaliação teórica e experimental destes algoritmos, utilizando dados simulados e também instâncias de aplicações práticas.

Os trabalhos resultaram em avanços teóricos no entendimento do problema U-curve e de uma de suas generalizações, assim como na introdução de três novos algoritmos para resolver este problema de forma ótima. Estes resultados serão apresentados ao longo desta tese. 


\subsection{Organização da tese}

Após esta introdução, este trabalho está organizado, nos próximos oito capítulos, da seguinte maneira:

- Capítulo 2 (Revisão bibliográfica e conceitos fundamentais): faremos uma revisão do estado da arte no problema de seleção de características. Apresentaremos algumas das funções custo mais empregadas, assim como diversos algoritmos e heurísticas propostos ao longo dos anos para resolver este problema. Apresentaremos um caso particular desse problema, o problema U-curve, que leva em consideração os casos em que a função custo é suscetível ao fenômeno da curva em U. Mostraremos ainda U-CuRve, um algoritmo homônimo ao problema e que foi proposto para resolver este último. Por fim, apresentaremos alguns conceitos e definições a serem utilizados ao longo deste trabalho, assim como uma descrição do formato dos experimentos.

- Capítulo 3 (O problema U-curve): descreveremos formalmente o problema U-curve. Apresentaremos uma prova de sua complexidade, algo que tem impacto direto nos algoritmos propostos para resolver esse problema.

- Capitulo 4 (O algoritmo U-Curve-SeArCh (UCS) ): mostraremos que o algoritmo U-Curve tem um erro que o torna sub-ótimo na resolução do problema U-curve. Introduziremos o algoritmo U-CURVE-SEARCH (UCS), o qual é ótimo para resolver o problema U-curve. Apresentaremos também uma análise de complexidade de tempo do algoritmo UCS, assim como alguns experimentos com o mesmo.

- Capítulo 5 (O problema da busca de início de caminho): verificaremos que a busca de um início de caminho (i.e., um elemento do espaço de busca corrente) no algoritmo UCS é feita de maneira ineficiente. Formalizaremos então o problema da busca de início de caminho, estudaremos a sua complexidade e apresentaremos duas abordagens para tentar resolvê-lo de forma mais sistemática.

- Capítulo 6 (Algoritmos branch-and-bound para o problema U-curve): apresentaremos dois novos algoritmos ótimos para resolver o problema U-curve: U-CURVE-BRANCH-AND-Bound (UBB), um algoritmo do tipo branch-and-bound, e também Poset-Forest-SEARCh (PFS), um algoritmo que generaliza UBB. Para ambos os algoritmos, forneceremos análise de complexidade de tempo e experimentos.

- Capítulo 7 (Experimentos): mostraremos alguns resultados experimentais com os algoritmos UCS, UBB e PFS. Faremos a análise dos mesmos a partir de experimentos ótimos e subótimos, que empregaram tanto dados simulados quanto dados reais obtidos de problemas práticos. Por fim, apresentaremos uma análise dos resultados obtidos.

- Capítulo 8 (Relaxamento do problema U-curve): apresentaremos uma proposta de relaxamento (generalização) do problema U-curve. Mostraremos que uma versão relaxada do problema U-curve serve para descrever o problema de seleção de características de um projeto de classificador, e que podemos utilizar os algoritmos UCS, UBB e PFS para resolvê-la.

- Capítulo 9 (Conclusões): faremos uma conclusão, na qual discutiremos sobre os resultados apresentados ao longo desta tese. Apontaremos as contribuições do trabalho, os artigos científicos produzidos durante o doutorado e também levantaremos algumas possibilidades de trabalhos futuros.

A partir do capítulo 3, todos os resultados são contribuições originais desta tese. 


\subsubsection{Sequências possíveis de leitura}

Os capítulos desta tese não precisam ser lidos na sequência em que são apresentados; por exemplo, após a leitura do capítulo 2, caso o leitor esteja interessado apenas nos experimentos comparativos entre os algoritmos apresentados neste trabalho, sem entrar nos detalhes dos mesmos, então ele poderá seguir diretamente para o capítulo 7. Na figura 1.2 apresentamos um fluxograma das ordens que o leitor poderá seguir ao estudar esta tese.

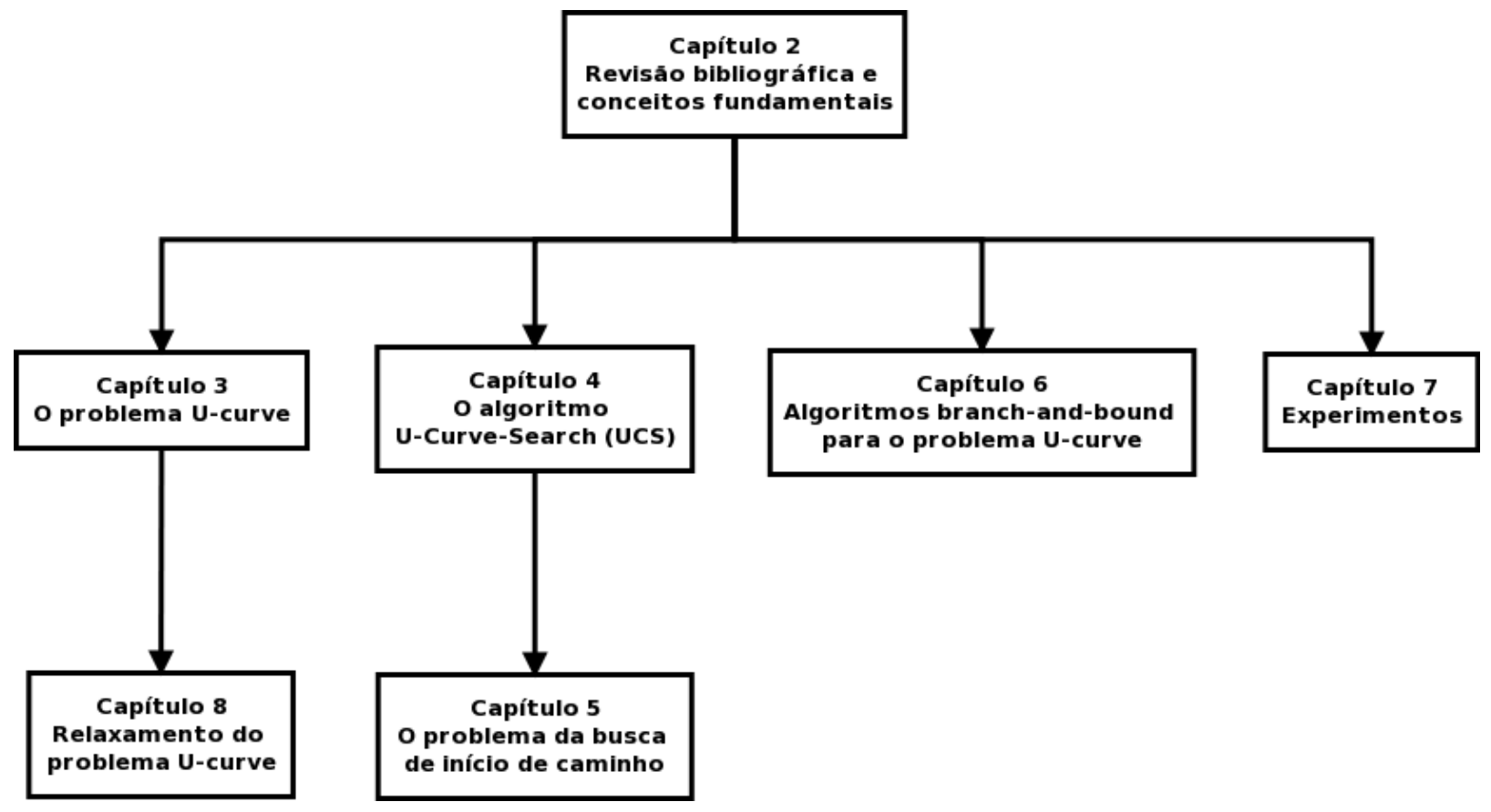

Figura 1.2: fluxograma que ilustra sequências possíveis de leitura desta tese. 


\section{Capítulo 2}

\section{Revisão bibliográfica e conceitos fundamentais}

Neste capítulo, apresentaremos uma revisão do estado da arte na resolução do problema de seleção de características, problema de grande interesse prático e que foi a motivação inicial deste trabalho. Analisaremos algumas funções custo empregadas para a resolução do problema, assim como alguns dos diversos algoritmos propostos ao longo dos anos, suas virtudes e limitações. Apresentaremos o caso particular do problema de seleção de caraterísticas estudado neste trabalho, o problema U-curve, assim como U-CuRVE, um algoritmo homônimo ao problema U-curve e que foi a primeira solução proposta na literatura para resolver este problema. Finalmente, introduziremos alguns conceitos e definições, assim como a estrutura em comum dos experimentos realizados em vários dos capítulos subsequentes.

\subsection{O problema de seleção de características}

Iniciaremos esta seção formalizando o problema de seleção de características e discutindo sobre algumas propriedades do mesmo. Em seguida, faremos uma revisão na literatura, levando em consideração a decomposição semântica de um problema de seleção de características em duas partes: função custo e algoritmo.

Seja $S$ um conjunto finito e não-vazio. O conjunto potência $\mathcal{P}(S)$ é a coleção de todos os subconjuntos de $S$, incluindo o conjunto vazio e o próprio $S$. Uma função custo (ou função critério, ou função objetivo) é uma função definida de $\mathcal{P}(S)$ para $\mathbb{R}+$.

Sejam $X$ um elemento de $\mathcal{X} \subseteq \mathcal{P}(S)$ e $c$ uma função custo. Se não existe um outro elemento $Y$ de $\mathcal{X}$ tal que $c(Y)<c(X)$, então $X$ é de custo mínimo em $\mathcal{X}$. Se $\mathcal{X}=\mathcal{P}(S)$, então nós dizemos simplesmente que $X$ é de custo mínimo.

Com estas definições, podemos definir o seguinte problema de busca.

Problema 2.1.1. (problema de seleção de características) Dados um conjunto finito e não-vazio $S$ e uma função custo c, encontrar um subconjunto $X$ de $S$ tal que $c(X)$ seja minimo.

O problema 2.1.1 é NP-difícil [JKP ${ }^{+}$94, GE03], o que implica que a busca de um subconjunto $X$ de $S$ tal que $c(X)$ seja mínimo pode ser intratável (i.e., pode levar um tempo computacional que é exponencial na cardinalidade de $S$ ). Entretanto, para algumas famílias de função custo existe garantia de que o problema não é NP-difícil e, consequentemente, de que o problema de busca seja tratável; por exemplo, quando a função custo é submodular [Sch00].

Não obstante, dependendo da definição da função custo, diferentes algoritmos podem ser empregados para resolver o problema de seleção de características. Dessa forma, a seleção de características depende de duas importantes etapas:

- a escolha de uma função custo apropriada, o que pode levar à definição de um problema de busca tratável ou intratável; 
- a escolha de um algoritmo de busca adequado para o problema de busca que foi definido. O algoritmo escolhido pode ser ótimo (i.e., ele sempre encontra um subconjunto de custo mínimo) ou sub-ótimo (i.e., ele pode não encontrar um subconjunto de custo mínimo).

\subsubsection{Funções custo de seleção de características}

O problema central na área de Reconhecimento de Padrões é projetar classificadores a partir de dados fornecidos por uma distribuição conjunta de um vetor de característica e de seus rótulos correspondentes [Ros98, MJCJB06, Rip08]. Geralmente, essa distribuição é estimada a partir de um número limitado de amostras e o erro de estimação depende da escolha do vetor de características.

Uma função custo de seleção de características é uma medida da distribuição conjunta estimada. Esta medida tem como objetivo identificar a disponibilidade de bons classificadores, isto é, de classificadores que cometam baixo erro na rotulação (classificação) no domínio em questão. Na literatura, existem várias funções custo de seleção de características disponíveis [DHS01, NF77]; dentre elas podemos destacar:

- a distância de divergência [DHS01];

- a distância Kullback-Leibler [TK06];

- o limitante (bound) de Chernoff [TK06];

- a distância de Bhattacharyya [TK06, NF77];

- a entropia condicional média [Lin91, MJCJB06].

Todas estas funções custo são medidas da estimação da distribuição conjunta de um subconjunto de características [BCJMJ+07, RBMJ10], desta forma elas são suscetíveis ao fenômeno da curva em $\mathrm{U}$ ( $U$-curve): para um número fixo de amostras, o aumento no número de características consideradas pode ter duas consequências: se o número de amostras disponíveis for suficiente para uma boa estimação, então deve ocorrer uma redução no erro de estimação; do contrário, a falta de dados induz a um aumento no erro de estimação. Se o número de amostras for grande o bastante para evitar o erro de estimação, então a medida é crescente, o que é explorado por alguns algoritmos ótimos do tipo branch-and-bound [NF77]. Entretanto, na prática, o número de dados disponíveis não são suficientes para boas estimações. Se uma função é suscetível ao fenômeno da curva em U e o domínio dessa função é restrito a uma cadeia do reticulado Booleano $(\mathcal{P}(S), \subseteq)$, então o gráfico do domínio restrito tem forma de U ( $U$-shaped graph). Uma função suscetível ao fenômeno da curva em U pode ser utilizada como uma função custo do problema U-curve, que por sua vez modela o problema de seleção de características.

\subsubsection{Algoritmos de seleção de características}

Um algoritmo de seleção de características é um algoritmo de busca no espaço $\mathcal{P}(S)$, utilizando como critério de avaliação a função custo $c$. Alguns mecanismos de um algoritmo de busca genérico são:

(i) visitar e medir um elemento $X$ de $\mathcal{P}(S)$;

(ii) comparar $c(X)$ com o mínimo atual e atualizar o mínimo se necessário;

(iii) realizar a poda (pruning) de cada elemento $Y$ de $\mathcal{P}(S)$ que seja conhecido que $c(Y)>c(X)$;

(iv) parar quando o mínimo atual satisfaz uma condição dada.

Até a presente data, não é conhecido um algoritmo polinomial para resolver o problema de seleção de características. Dessa forma, algumas heurísticas que produzem resultados sub-ótimos são aplicadas neste problema NP-difícil [PNK94, $\mathrm{SPN}^{+}$99, NC09]. 
Um dos primeiros algoritmos de seleção de características a surgir foi a seleção sequencial (sequential selection): em 1963, Marill e Green apresentaram um algoritmo de seleção de características para instâncias cuja função custo fosse determinada pela distância de divergência (por exemplo, a distância euclidiana). Este algoritmo guloso, chamado de SBS (Sequential Backward Selection, ou "busca sequencial para trás"), inicia sua execução definindo um conjunto $X:=S$; a cada iteração, SBS remove um elemento $x$ de $X$, de tal modo que a distância entre $X$ e $X-\{x\}$ seja máxima [MG63]. Observe que o algoritmo SBS é equivalente a uma busca em profundidade no espaço de busca, porém sem backtracking [SS93]. Em 1971, Whitney introduziu o algoritmo dual de SBS, chamado de SFS (Sequential Forward Selection, ou "busca sequencial para frente") [Whi71]. Embora seja polinomial, o algoritmo SBS (no caso dual, SFS) sofre o chamado "efeito de aninhamento" (nesting effect): ou seja, que uma vez que seja excluído (incluído) um elemento de (em) $X$, o mesmo jamais incluído em (removido de) $X$. Portanto, não há como garantir a seleção de um conjunto ótimo [PNK94].

Alguns métodos foram propostos para tentar resolver o problema do efeito de aninhamento dos algoritmos SBS e SFS [Ste76, Kit78]. Em 1973, Michael e Lin apresentaram um algoritmo com essa finalidade [ML73], que posteriormente foi refinado por Stearns no algoritmo PLUS-L-MinUS-R [Ste76]. O algoritmo Plus-L-Minus-R mitiga o problema de aninhamento, porém tem como ponto fraco a impossibilidade de se estimar os valores dos parâmetros utilizados para controlar a inspeção do espaço de busca, de forma a permitir o alcance de uma solução ótima. Visando mitigar esse fato, Kittler apresentou uma generalização de SBS, SFS e PLus-L-Minus-R [Kit78]. As pesquisas envolvendo métodos sequenciais atingiram um patamar importante quando, em 1994, Pudil e colegas introduziram o algoritmo Sequential Forward Floating Selection, mais conhecido como SFFS [PNK94]. SFFS (e sua versão dual, SBFS) deixa os valores dos parâmetros de controle do espaço de busca "flutuarem" ao longo das iterações do algoritmo, o que ajuda a mitigar o efeito de aninhamento e melhora os resultados práticos [SS93, PNK94].

Ao longo dos últimos anos, diversos trabalhos foram publicados sugerindo melhorias no algoritmo SFFS; entre eles está o Adaptative Floating Search, chamado de algoritmo ASFFS, introduzido em 1999 por Somol e colegas [SPN ${ }^{+}$99]. ASFFS pode melhorar a qualidade do conjunto final, por se utilizar de um recálculo mais sofisticado dos parâmetros de controle do espaço de busca, porém às custas de um maior tempo computacional [SPN ${ }^{+}$99]. Outros algoritmos derivados do SFFS, mais recentes, foram introduzidos em 2006 por Somol e colegas (Flexible-hybrid SFFS- HSFFS [SNP06]) e em 2009 por Nakariyakul e colegas (Improved SFFS - ISFFS [NC09]).

Todos os algoritmos apresentados até aqui são da classe dos algoritmos de seleção sequencial, e que têm como característica serem determinísticos e de fornecerem, em tempo polinomial, uma única solução, sem a garantia da mesma ser um ótimo global [JZ97]. Um algoritmo estocástico que também fornece uma solução sub-ótima em tempo polinomial é o algoritmo de recozimento simulado (simulated annealing), como por exemplo o apresentado, em 1989, por Siedlecki e Sklansky [SS89]. O algoritmo de recozimento simulado pode ser entendido como uma versão aleatorizada do algoritmo Plus-L-Minus-R [SS89].

Algoritmos sub-ótimos que fornecem múltiplas soluções em tempo polinomial também foram propostos. Siedlecki e Sklansky introduziram, em 1989, o algoritmo BEAM-SEARCH [SS89]. Esse algoritmo utiliza uma fila de possíveis soluções, utilizando os conjuntos dessa fila para realizar buscas em profundidade sem backtracking [JZ97]. O algoritmo SBS pode ser visto como um caso particular de BEAM-SEARCH, com uma fila de tamanho um [SS89]. Uma outra propriedade de BEAM-SEARCH é que este algoritmo trata o espaço de busca como um grafo, chamado de reticulado de seleção de características; porém, essa forma de abordar o problema não era comum na literatura até meados dos anos 90 [JZ97]. Por fim, uma versão aleatorizada do algoritmo BEAM-SEARCH, equivalente a um algoritmo genético, foi apresentada em 1989 por Siedlecki e Sklansky [SS89].

Os algoritmos de seleção de características que garantem a otimalidade da solução constituem uma família muito menos explorada na literatura [PNK94, JZ97]. A maneira mais simples de resolver o problema de características de forma ótima é a realização de uma busca exaustiva naive; todavia, tal procedimento pode ser inviável mesmo para instâncias não muito grandes. Uma nova abordagem 
para resolver o problema de seleção de características e que traz garantia de uma solução ótima foi introduzida em 1977 por Narendra e Fukunaga; essa abordagem utiliza um algoritmo do tipo branch-and-bound [NF77]. Tal qual na busca exaustiva, o algoritmo garante que a solução fornecida é ótima; porém, o algoritmo supõe que a função custo do problema de seleção de características seja monotônica. Ademais, o algoritmo pode ser impraticável para instâncias muito grandes [PNK94, RBMJ10].

Na figura 2.1 é exibida uma árvore contendo alguns dos algoritmos apresentados nesta revisão. Os algoritmos estão organizados por categoria, isto é, se o algoritmo garante ou não uma solução ótima, se ele é determinístico ou estocástico e se devolve uma ou várias soluções.

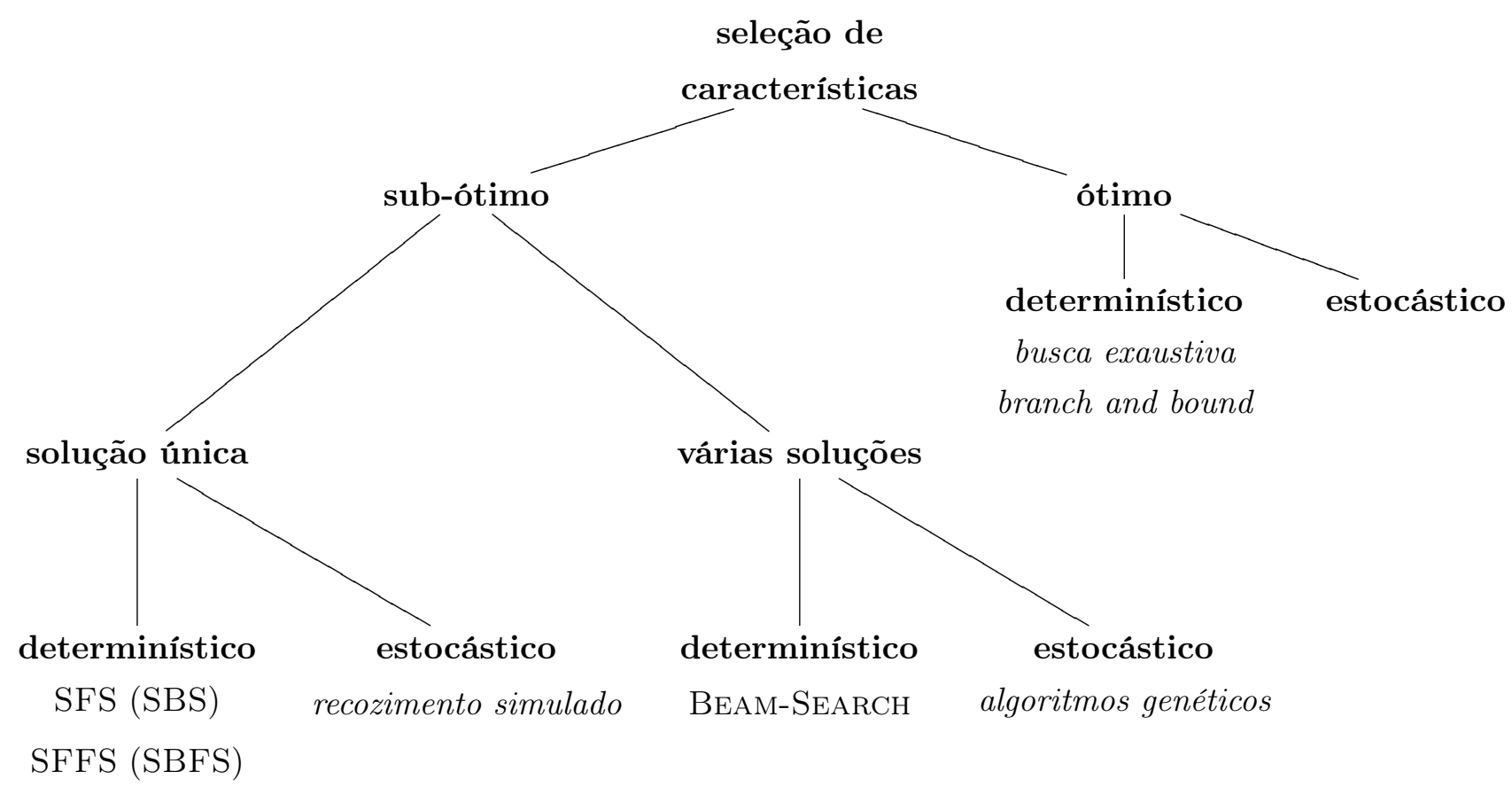

Figura 2.1: a taxonomia de alguns algoritmos de seleção de características utilizados em Reconhecimento de Padrões. Adaptado de Jain e colegas, 1997 [JZ97].

\subsubsection{Um caso particular: o problema U-curve}

Como vimos na revisão de funções custo de seleção de características, diversos problemas de seleção de características podem ser reduzidos a um problema de busca em que a função custo a ser minimizada é suscetível ao fenômeno da curva em U. Este subconjunto da família dos problemas de seleção de características, também conhecido como problema $U$-curve, pode ser utilizado para descrever a etapa de seleção de características de importantes aplicações práticas em Reconhecimento de Padrões, como por exemplo a identificação de preditores na estimação de Redes Gênicas Probabilísticas [BCJMJ+ 04, $\mathrm{BCJMJ}^{+}$07] e o projeto de W-Operadores [MJCJB04].

A seguir, apresentaremos um algoritmo proposto para resolver de forma ótima o problema Ucurve.

\section{O algoritmo U-CuRve}

Visando resolver o problema U-curve, em 2010 Ris e colegas introduziram U-CuRVE, um algoritmo homônimo ao problema [RBMJ10]. U-CURVE foi proposto como um algoritmo ótimo para resolver o problema U-curve e tem as seguintes propriedades: 
(i) opera sobre um espaço de busca (i.e., a coleção de subconjuntos de um conjunto finito e não-vazio) que é um reticulado Booleano;

(ii) otimiza funções custo que são suscetíveis ao fenômeno da curva em U.

A maneira na qual o algoritmo U-CuRve visita o espaço de busca leva em consideração as propriedades (i) e (ii): a cada iteração, o algoritmo explora uma cadeia maximal, seja em um percorrimento de baixo para cima ou de cima para baixo, até que ele atinja um elemento $M$ que tenha o menor custo entre os elementos dessa cadeia. Então, o algoritmo executa um procedimento chamado "esgotamento do mínimo", que faz uma busca em profundidade (depth-first search) por elementos de custo menor ou igual a $c(M)$. Ao final de uma iteração, é atualizada uma lista contendo os elementos de custo mínimo explorados até então, assim como o espaço de busca é reduzido através da atualização de duas coleções de elementos chamadas "restrições inferiores" e "restrições superiores". Cada elemento $L$ das "restrições inferiores" remove do espaço de busca os elementos contidos em $L$, enquanto que cada elemento $U$ das "restrições superiores" remove do espaço de busca os elementos que contêm $U$. O algoritmo realiza uma sucessão de iterações até que o espaço de busca se torne vazio. Para uma explicação detalhada da dinâmica de U-CuRVE, consulte o trabalho de Ris e colegas [RBMJ10], em particular a figura 4.

O algoritmo U-CURVE mostrou um melhor desempenho que a heurística SFFS; isto é, para uma dada instância do problema U-curve, U-CURVE encontra subconjuntos de custo menor ou igual ao custo do melhor subconjunto encontrado por SFFS, geralmente com menos acessos à função custo [RBMJ10]. Evitar acessos desnecessários à função custo é, em geral, importante em problemas de seleção de características, tendo em vista que frequentemente um acesso é caro e o espaço de busca é muito grande.

Na figura 2.3 mostramos qual é a classificação do algoritmo U-CuRve na árvore de algoritmos de seleção de características exibida na figura 2.1. Já na figura 2.3, apresentamos um exemplo típico da topologia de percorrimento do espaço de busca de U-CuRve e também de alguns outros algoritmos de seleção de características.

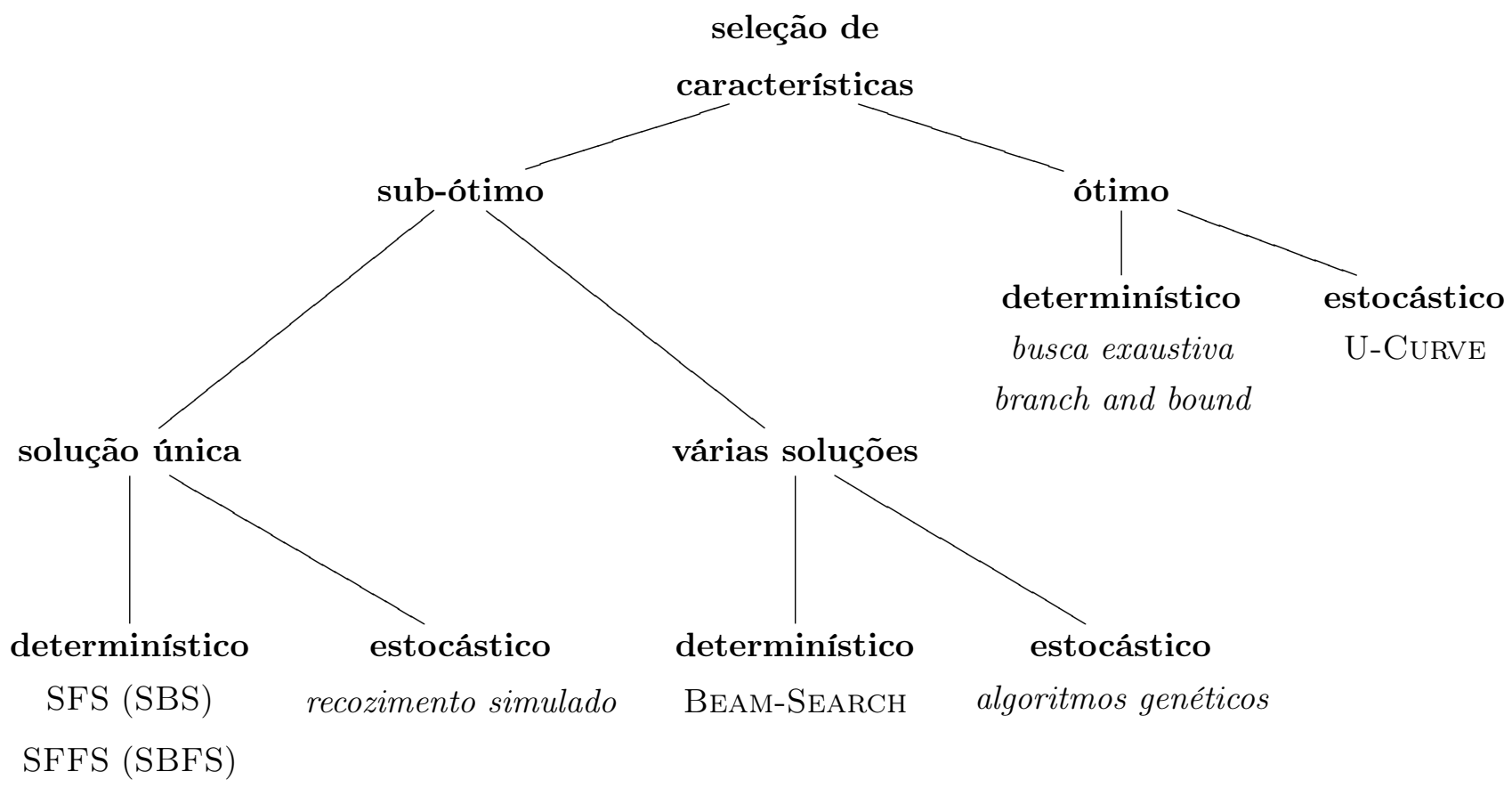

Figura 2.2: a localização do algoritmo U-CuRve na taxonomia de algoritmos de seleção de características exibida na figura 2.1 - U-CuRve é um algoritmo que foi projetado com a intenção de ser ótimo e estocástico. 


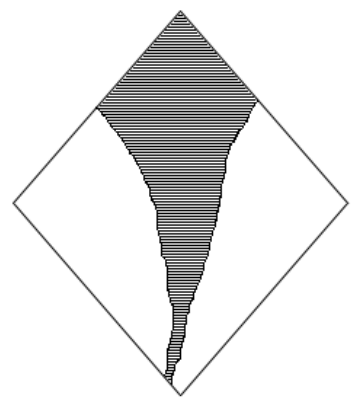

SBS

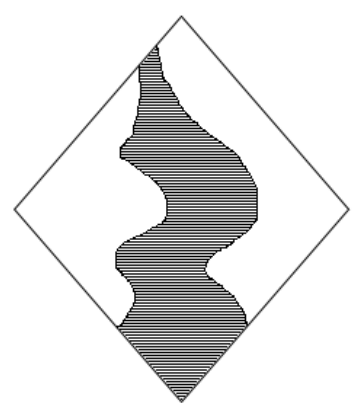

SFFS

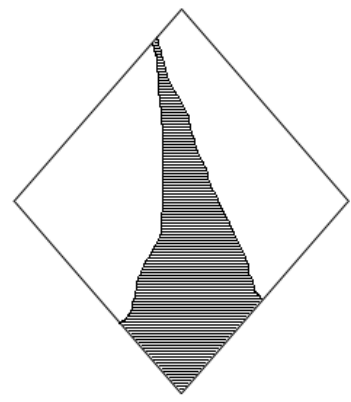

SFS

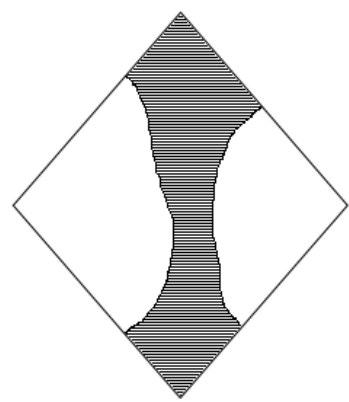

Busca bidirecional

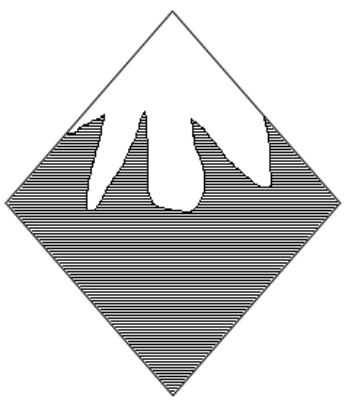

Branch-and-bound

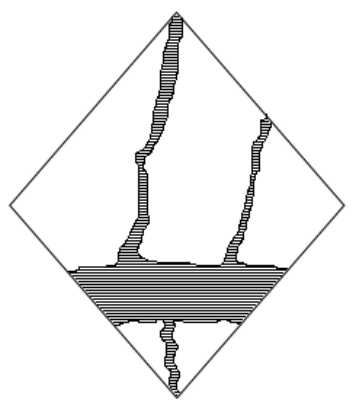

U-Curve

Figura 2.3: os caminhos percorridos em um reticulado Booleano por diferentes algoritmos de busca. "Busca bidirecional" consiste na execução dos algoritmos SBS e SFS, na qual toma-se uma melhor solução obtida por ambos. Adaptado de Siedlecki e Sklansky [SS93].

\subsection{Conceitos fundamentais}

Nos capítulos seguintes deste trabalho apresentaremos diversos algoritmos ótimos que abordam o problema U-curve. Todos eles utilizam conceitos e descrições que serão apresentados nesta seção. Iniciaremos introduzindo alguns conceitos e uma descrição da sistemática de apresentação dos algoritmos; em seguida, apresentaremos a estrutura comum a todos os experimentos realizados: o arcabouço utilizado para implementar os algoritmos, o algoritmo de busca exaustiva elaborado para fins de comparação com os demais algoritmos desenvolvidos, os tipos de instâncias empregadas nos experimentos (de dados reais e simulados) e os tipos de experimentos realizados (ótimos e sub-ótimos).

A partir da leitura desta seção, o leitor poderá seguir a sequência de capítulos, ou então ir diretamente para os capítulos 4-5 (algoritmo UCS e seus melhoramentos), para o capítulo 6 (algoritmos branch-and-bound para o problema U-curve), ou ainda para o capítulo 7 (experimentos com todos os algoritmos, utilizando instâncias de dados reais e simulados).

\subsubsection{Definições iniciais}

Conjuntos e vetor característico. Seja $S$ um conjunto finito e não-vazio. A cardinalidade de $S$ será denotada por $n:=|S|$. O conjunto potência $\mathcal{P}(S)$ também é denominado espaço de busca. Suporemos que cada elemento do espaço de busca $\mathcal{P}(S)$ utilize $n$ bits para representá-lo; tal suposição é razoável, uma vez que um elemento de $\mathcal{P}(S)$ pode ser representado por uma bijeção de $\mathcal{P}(S)$ para $\{0,1\}^{n}$.

Seja $S$ um conjunto finito ordenado e $A$ um subconjunto de $S$. Denotamos por $\chi^{A}$ o vetor característico de $A$, definido como 


$$
\chi_{s}^{A}= \begin{cases}1, & \text { se } s \in A \\ 0, & \text { se } s \in S-A,\end{cases}
$$

para todo $s \in S$. Na maior parte das figuras deste trabalho que ilustram reticulados Booleanos, todos os elementos dos mesmos são apresentados através de seus vetores característicos. Por exemplo, se $S=\{a, b\}$ e $A=\{a\}$, então $\chi^{A}=10$; num abuso de notação, muitas vezes escreveremos que $A=10$.

Intervalos. Sejam $A$ e $B$ elementos de $\mathcal{P}(S)$. Um intervalo $[A, B]$ de $\mathcal{P}(S)$ é definido como $[A, B]:=\{X \in \mathcal{P}(S): A \subseteq X \subseteq B\}$. Seja $[\emptyset, L]$ um intervalo cujo elemento mais à esquerda é o conjunto vazio. $L$ é uma restrição inferior. Do mesmo modo, seja $[U, S]$ um intervalo cujo elemento mais à direita é o conjunto completo. $U$ é uma restrição superior.

Grafos. Um grafo $G$ é um par ordenado de dois conjuntos $(V, E)$, composto de um conjunto $V$ de vértices e de um conjunto $E$ de arestas cujos elementos são subconjuntos de dois elementos de $V$. Podemos nos referir aos conjuntos de vértices e de arestas de um grafo $G$ por $V_{G}$ e $E_{G}$, respectivamente.

Seja $G=(V, E)$ um grafo. Se uma aresta $e$ contém um vértice $v$, então dizemos que $e$ é incidente em $v$. Uma cobertura de vértices (vertex cover) de um grafo $G$ é um subconjunto $K \subseteq V$ de vértices de $G$ tal que cada aresta de $G$ é incidente em ao menos um vértice de $K$. Seja $K$ uma cobertura de vértices de um grafo $G$. $K$ é mínima se não existe outra cobertura de vértices de $G$, digamos $K^{\prime}$, tal que $|K|>\left|K^{\prime}\right|$. Na figura 2.4 mostramos um grafo $G$ com cinco vértices e sete arestas, assim como uma cobertura de vértices de $G$.

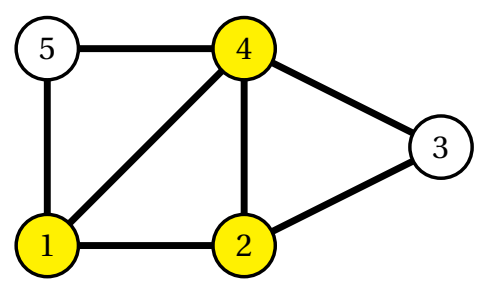

Figura 2.4: um grafo $G$ com cinco vértices e sete arestas. Os vértices em amarelo constituem uma cobertura de vértices de $G$; tal cobertura é mínima.

Se os vértices de um grafo $G$ são elementos de $\mathcal{P}(S)$ e as arestas de $G$ são os pares de elementos de $\mathcal{P}(S)$ que têm distância da Hamming igual a um, então $G$ é um hipercubo de grau $|S|[H H W 88]$.

Um sub-grafo $H$ de um grafo $G$ é um grafo tal que $V_{H} \subseteq V_{G}$ e $E_{H} \subseteq E_{G}$. Um sub-grafo $H$ de $G$ pode ser indicado pela expressão $H \subseteq G$. Um caminho $P$ de $G$ é um sub-grafo de $G$ que admite uma permutação $\left\langle v_{1} \ldots v_{\left|V_{P}\right|}\right\rangle$ dos elementos de $V_{P}$ tal que

$$
\left\{\left\{v_{i}, v_{i+1}\right\}: 1 \leq i<\left|V_{P}\right|\right\}=E_{P} .
$$

Dizemos que $v_{1}$ e $v_{\left|V_{P}\right|}$ são os extremos do caminho $P$. Se $\left|V_{P}\right| \geq 3$ e $G$ possui a aresta $\left\{v_{1}, v_{\left|V_{P}\right|}\right\}$, então dizemos que o sub-grafo $\left(V_{P}, E_{P} \cup\left\{v_{1}, v_{\left|V_{P}\right|}\right\}\right)$ é um circuito de $G$.

Seja $G$ um grafo. $G$ é conexo se todos pares de vértices de $G$, distintos dois a dois, são extremos de um caminho de $G$. Se $G$ não é conexo então dizemos que o mesmo é desconexo.

Uma árvore é um grafo conexo que não possui circuitos.

Algoritmos. Seja Algorithm um algoritmo. A descrição de Algorithm será feita seguindo uma decomposição lógica em uma abordagem de baixo para cima, isto é, as sub-rotinas serão apresentadas e estudadas na ordem inversa da árvore de chamada das mesmas, na qual ALGORITHM é a raiz. A apresentação de cada sub-rotina será feita na seguinte ordem: primeiro, a descrição da 
sub-rotina e seu pseudo-código; segundo, a prova de sua corretude (quando houver); terceiro, sua análise de complexidade de tempo. Os pseudo-códigos são omitidos sempre que eles forem simples. Uma vez que a complexidade de tempo de algoritmos duais é a mesma, ela será apresentada apenas uma vez.

Complexidade de tempo da função custo. Para todo algoritmo descrito neste trabalho, qualquer chamada da função custo utilizada exige $O(f(n))$ unidades de tempo. $f(n)$ é definida de acordo com a função custo do problema explorado, o que implica que as análises de complexidade de tempo dos algoritmos tornam-se independentes de um tipo específico de função custo.

\subsubsection{Estrutura dos experimentos realizados}

\section{$\mathrm{O}$ arcabouço featsel}

Para realizar a avaliação experimental de todos os algoritmos deste trabalho, desenvolvemos featsel, um arcabouço orientado a objetos codificado em $\mathrm{C}++$, que permite implementação de funções custo definidas sobre reticulados Booleanos, assim como de algoritmos que fazem uso de tais funções e que utilizam reticulados Booleanos como espaço de busca [Rei12]. Todo algoritmo e toda função custo apresentados neste trabalho foram implementados utilizando este arcabouço. No apêndice A apresentamos maiores detalhes de featsel.

Todos os experimentos deste trabalho foram realizados em um PC de 32 bits com relógio (clock) de $2.8 \mathrm{GHz}$ e $8 \mathrm{~GB}$ de memória. Em todos os experimentos mostrados neste trabalho, a memória do computador utilizado para realizá-los era muito maior do que o tamanho da maior instância (i.e., respectivamente, 8 GB e $256 \mathrm{~KB}$ ); portanto, a memória disponível não foi um limitante para o desempenho dos algoritmos.

\section{Algoritmo de busca exaustiva}

Para realizar análises comparativas dos algoritmos deste trabalho, desenvolvemos um algoritmo de busca exaustiva que consome $O\left((f(n)+n) 2^{n}\right)$ unidades de tempo. Exhaustive-Search é utilizado nos capítulos 4 e 6 e é descrito no apêndice B.

\section{Instâncias empregadas}

Em todos os experimentos deste trabalho foram empregados um dos seguintes tipos de instâncias:

- dados simulados de instâncias difíceis;

- dados reais de projeto de $\mathrm{W}$-operadores.

Simulação de instâncias difíceis. Instâncias simuladas foram utilizadas para avaliar o desempenho dos algoritmos em casos em que as mesmas são "difíceis". Para um teste com um conjunto não-vazio $S$ de tamanho $n$, as instâncias foram obtidas através da geração de $n$ inteiros aleatórios e de um inteiro $t$ no intervalo [0, $\left.\sum_{s \in S} s\right]$ e, finalmente, fixando a função custo $c$ como a equação 3.1. Como será mostrado na prova do lema 3.2.2 (capítulo 3), $c$ é uma redução polinomial do problema da soma de subconjuntos, o que implica que $c$ é uma função custo de um problema NP-difícil.

Para realizar experimentos com instâncias simuladas, produzimos 100 instâncias aleatórias para cada tamanho $n$ de instância, $7 \leq n \leq 18$.

Projeto de W-operadores. Neste caso, trataram-se de instâncias de dados reais, obtidos a partir de amostras de pares de imagens durante o projeto de W-operadores. Como estas instâncias foram utilizadas apenas em experimentos do capítulo 7, a maneira como estas instâncias foram utilizadas (assim como a função custo empregada) será explicada nesse último. 


\section{Tipos de experimentos}

Para este trabalho, realizamos dois tipos de experimentos:

- os ótimos, nos quais os algoritmos são avaliados como algoritmos de busca ótimos;

- os sub-ótimos, nos quais os algoritmos são avaliados como heurísticas, cujo critério de parada é um limiar superior para o número de vezes que a função custo pode ser computada; tal limiar é obtido através de etapas de pré-processamento que serão explicadas a seguir.

Experimentos ótimos. Neste tipo de experimento, para cada grupo de instâncias de mesmo tamanho, executamos todos os algoritmos descritos neste trabalho em cada instância do mesmo grupo; para cada algoritmo, calculamos a média do tempo de execução, a média do número de nós computados (i.e., o número de vezes que a função custo foi computada) e o número de vezes que o algoritmo encontrou uma melhor solução (neste caso, uma solução ótima).

Experimentos sub-ótimos. Os experimentos sub-ótimos são feitos em três etapas: as duas primeiras são um pré-processamento, feito para produzir um critério para parar a busca; a terceira etapa é a busca sub-ótima de fato, que utiliza o critério de parada obtido nas etapas de préprocessamento. A seguir, descrevemos como cada etapa é executada:

- primeiro, a heurística SFFS é executada sobre um grupo de instâncias de mesmo tamanho. Em seguida, é calculada a média do custo da melhor solução obtida em cada uma das instâncias;

- segundo, os algoritmos avaliados são executados sobre o mesmo grupo de instâncias, desta vez utilizando como critério de parada o limiar obtido na primeira etapa. Desta forma, para cada algoritmo, é calculada a média do número de vezes em que a função custo é computada; dentre as médias obtidas, a maior será utilizada como limiar para a próxima etapa;

- terceiro, os algoritmos avaliados são executados uma vez mais sobre o mesmo grupo de instâncias, desta vez utilizando como critério de parada o limiar obtido na segunda etapa.

Finalmente, os resultados são organizados na mesma maneira apresentada nos experimentos ótimos.

Neste trabalho optamos por utilizar como critério de parada dos experimentos sub-ótimos o número de vezes que a função custo é computada ao invés de um tempo arbitrário de execução. A principal razão dessa opção foi garantir que os algoritmos fossem bem definidos e que os resultados dos experimentos sejam reproduzíveis (Johnson, pet peeve 8 [Joh02]). 


\section{Capítulo 3}

\section{O problema U-curve}

Mostraremos neste capítulo um estudo sobre a complexidade do problema U-curve, um caso particular do problema de seleção de características (problema 2.1.1). Iniciaremos apresentando uma descrição formal do problema U-curve. Em seguida, mostraremos que este problema de fato é NP-difícil. Concluiremos o capítulo fazendo algumas observações sobre os resultados obtidos.

\subsection{Formalização do problema U-curve}

Seja $S$ um conjunto finito e não vazio. O conjunto parcialmente ordenado (poset) $(\mathcal{P}(S), \subseteq)$ é um reticulado Booleano de grau $|S|$ no qual o maior e o menor elemento são, respectivamente, $\emptyset$ e $S$, e a soma e o produto são, respectivamente, a união e a interseção usuais sobre conjuntos.

Uma cadeia é uma coleção $\left\{X_{1}, X_{2}, \ldots, X_{k}\right\} \subseteq \mathcal{P}(S)$ tal que $X_{1} \subseteq X_{2} \subseteq \ldots \subseteq X_{k}$. Uma cadeia de $\mathcal{P}(S)$ é maximal se não é contida propriamente por outra cadeia de $\mathcal{P}(S)$. Toda cadeia maximal contém os conjuntos $\emptyset$ e $S$ e tem $|S|+1$ elementos.

Sejam $\mathcal{X} \subseteq \mathcal{P}(S)$ uma cadeia e $f$ uma função que leva valores de $\mathcal{X}$ para $\mathbb{R}+. f$ descreve uma curva em U se, para quaisquer $X_{1}, X_{2}, X_{3} \in \mathcal{X}, X_{1} \subseteq X_{2} \subseteq X_{3}$ implica que $f\left(X_{2}\right) \leq$ $\max \left\{f\left(X_{1}\right), f\left(X_{3}\right)\right\}$. Seja $c$ uma função custo (i.e., uma função que leva valores de $\mathcal{P}(S)$ para $\mathbb{R}+)$. c é decomponível em curvas em $\mathbf{U}$ se, para toda cadeia maximal $\mathcal{X} \subseteq \mathcal{P}(S)$, a restrição de $c$ a $\mathcal{X}$ descreve uma curva em $\mathrm{U}$.

Agora vamos definir o problema central que é estudado neste trabalho.

Problema 3.1.1. (problema U-curve) Dados um conjunto finito e não-vazio $S$ e uma função custo $c$ decomponivel em curvas em $U$, encontrar um subconjunto $X$ de $S$ tal que $c(X)$ seja mínimo.

Na seção a seguir faremos uma análise da complexidade do problema 3.1.1.

\subsection{Análise de complexidade do problema U-curve}

Agora vamos estudar a complexidade do problema 3.1.1 (problema U-curve). Para isso, formulamos um problema de decisão relacionado ao problema U-curve.

Problema 3.2.1. (problema de decisão U-curve) Dados um conjunto finito e não-vazio S, uma função custo c decomponivel em curvas em $U$ e um número inteiro $k$, decidir se existe um subconjunto $X$ de $S$ tal que $c(X)$ é menor ou igual a $k$.

O problema de decisão U-curve é relacionado ao problema U-curve, no sentido que uma instância $\langle S, c, k\rangle$ do problema de decisão U-curve pode ser resolvida utilizando os valores de $S$ e $c$ para resolver o problema U-curve e comparando o valor de um subconjunto de custo mínimo obtido com $k$. Portanto, o problema U-curve é ao menos tão difícil quanto o problema de decisão U-curve. Como demonstraremos a seguir, é pouco provável que o problema de decisão U-curve tenha uma solução eficiente. 
No lema a seguir, por suposição um valor $c(X)$ é fornecido por um oráculo em um tempo que é polinomial no tamanho de $S$. Para provar este lema, mostraremos que existe uma injeção do conjunto de instâncias de conhecidos problemas NP-completos no conjunto de instâncias do problema de decisão U-curve.

Lema 3.2.2. O problema de decisão U-curve é NP-completo.

Provaremos o lema 3.2.2 utilizando uma redução polinomial a partir do problema da soma de subconjuntos (subset sum problem, Cormen et al. [CLRS01], página 1013).

Demonstração. Iniciamos a prova verificando que o problema de decisão U-curve pertence a NP: devido ao tempo exigido pelo oráculo, é possível verificar em um tempo polinomial em $|S|$ se um subconjunto $X$ de $S$ tem custo menor ou igual a $k$.

Para mostrar que o problema de decisão U-curve é NP-difícil, provaremos que ele é ao menos tão difícil quanto o problema da soma de subconjuntos. No problema da soma de subconjuntos, dados um conjunto finito $S$ de inteiros não-negativos e um inteiro não-negativo $t$, queremos saber se existe um subconjunto $X$ de $S$ tal que a soma dos elementos de $X$ seja exatamente $t$. Dada uma instância $\langle S, t\rangle$ do problema da soma de subconjuntos, construiremos uma instância $\left\langle S^{\prime}, c, k\right\rangle$ do problema de decisão U-curve, em um tempo limitado por um polinômio em $|S|$. Esta instância é tal que existe um subconjunto $X$ de $S$ cuja soma é igual a $t$ se e somente se existe um subconjunto $X^{\prime}$ de $S$ cujo custo é menor ou igual a $k$. O conjunto $S^{\prime}$ é definido como uma cópia de $S$, o valor de $k$ é definido como zero, e a função custo é:

$$
c\left(X^{\prime}\right)=\left|t-\sum_{x \in X^{\prime}} x\right| \text {, para todo } X^{\prime} \subseteq S^{\prime} .
$$

Agora provaremos que $c$ é uma função custo decomponível em curvas em $\mathrm{U}$, isto é, que para cada cadeia $\mathcal{C} \subseteq \mathcal{P}\left(S^{\prime}\right)$, para quaisquer $A, B, C \in \mathcal{C}, A \subseteq B \subseteq C$ implica que $c(B) \leq \max \{c(A), c(C)\}$. Consideremos dois casos. No primeiro caso, seja $B \in \mathcal{P}\left(S^{\prime}\right)$ tal que $\sum_{b \in B} b<t$. Então, para qualquer $A \subseteq B$ temos que:

$$
\begin{aligned}
c(B) & =\left|t-\sum_{b \in B} b\right| \\
& \leq\left|t-\sum_{b \in B} b+\sum_{d \in D} d\right| \\
& =\left|t-\sum_{a \in A} a\right| \\
& =c(A)
\end{aligned}
$$$$
\text { (uma vez que } t-\sum_{b \in B} b>0 \text { ) }
$$$$
\text { (uma vez que } A \subseteq B \text { implica que } A=B-(B-A) \text { ) }
$$$$
\text { (pela definição de } c \text { ). }
$$

No segundo caso, seja $B \in \mathcal{P}\left(S^{\prime}\right)$ tal que $\sum_{b \in B} b \geq t$. Então, para qualquer $C \supseteq B$ temos que:

$$
\begin{aligned}
c(B) & =\left|t-\sum_{b \in B} b\right| \\
& \leq\left|t-\sum_{b \in B} b-\sum_{e \in E} e\right| \\
& =\left|t-\sum_{c \in C} c\right| \\
& =c(C)
\end{aligned}
$$$$
\text { (uma vez que } t-\sum_{b \in B} b \leq 0 \text { ) }
$$$$
\text { (uma vez que } B \subseteq C \text { implica que } C=B \cup(C-B) \text { ) }
$$

(pela definição de $c$ ).

Dessa forma, ou $A \subseteq B$ implica que $c(B) \leq c(A)$ ou $B \subseteq C$ implica que $c(B) \leq c(C)$, o que leva ao fato que $A \subseteq B \subseteq C$ implica que $c(B) \leq \max \{c(A), c(C)\}$. Portanto, concluímos que a função custo $c$ é decomponível em curvas em $\mathrm{U}$.

Finalmente, precisamos mostrar que existe um subconjunto $X$ de $S$ cuja soma é igual a $t$ se e somente se existe um subconjunto $X^{\prime}$ de $S$ cujo custo é menor ou igual a $k$. Mostramos que se 
existe um subconjunto $X$ de $S$ cujo custo é igual a $t$, então existe um subconjunto $X^{\prime}$ de $S^{\prime}$ tal que $X^{\prime}=X$ e, por definição de $c$, o valor de $c(X)$ é $0 \leq k$. Inversamente, se existe um subconjunto $Y^{\prime}$ de $S^{\prime}$ tal que $c\left(Y^{\prime}\right) \leq k=0$, então o valor de $c\left(Y^{\prime}\right)$ é zero, pela definição de $c$. Ademais, devido a $S=S^{\prime}, c\left(Y^{\prime}\right)=0$, e pela definição de $c$, existe um subconjunto $Y$ de $S$ tal que $Y=Y^{\prime}$ e a soma de seus elementos é exatamente $t$.

Apresentaremos agora o resultado principal desta seção.

Teorema 3.2.3. O problema U-curve é NP-difícil

Demonstração. O fato do problema de decisão U-curve ser NP-completo (Lema 3.2.2) implica que o problema U-curve, que é um problema de otimização relacionado, é NP-difícil.

\subsection{Comentários}

Neste capítulo provamos que o problema de otimização U-curve (problema 3.1.1) é NP-difícil. Além disso, vale observar que a redução polinomial empregada na demonstração do lema 3.2.2 (i.e., a que reduz uma instância do problema da soma de subconjuntos para uma instância do problema U-curve, utilizando para isso a equação 3.1) é linear no tamanho do conjunto $S$; dessa forma, aproveitaremos essa redução polinomial para gerar instâncias simuladas "difíceis" do problema Ucurve.

Uma possibilidade, a ser investigada no futuro, é a utilização de algoritmos que resolvam o problema U-curve para atacar algumas famílias de instâncias de problemas NP-difíceis tais como, por exemplo, famílias de instâncias do próprio problema da soma de subconjuntos ou de problemas relacionados (e.g., o problema da mochila). 


\section{Capítulo 4}

\section{O algoritmo U-CURVE-SEARCH (UCS)}

No capítulo anterior formalizamos o problema de otimização U-curve (problema 3.1.1). Também demonstramos, através do teorema 3.2.3, que este problema é NP-difícil. Como já foi visto no capítulo 2, Ris e colegas propuseram U-CuRVE, um algoritmo que visava resolver de forma ótima o problema U-curve [RBMJ10]. Todavia, como será demonstrado neste capítulo, a solução proposta por Ris e colegas possui um erro que leva a resultados sub-ótimos. Tal constatação justificou o desenvolvimento de um novo algoritmo realmente ótimo para resolver o problema U-curve.

Iniciaremos este capítulo mostrando o problema existente em U-CURVE. Em seguida, apresentaremos U-CURVE-SEARCH (UCS), um algoritmo ótimo para resolver o problema U-curve. Apresentaremos os princípios de funcionamento do novo algoritmo, pseudo-código do mesmo e de suas sub-rotinas, assim como análises de complexidade de tempo computacional. Em seguida, serão mostrados alguns experimentos feitos com o algoritmo e análises sobre os resultados obtidos. Por fim, concluiremos fazendo alguns comentários sobre possíveis trabalhos futuros com o algoritmo apresentado neste capítulo.

\subsection{Problema com o algoritmo U-CuRVE}

O algoritmo U-CuRve foi desenvolvido com a intenção de ser ótimo na resolução do problema U-curve [RBMJ10]. Não obstante, uma análise de sua correção resultou na descoberta de um problema que leva a resultados sub-ótimos em alguns tipos de instâncias. Este problema está localizado na sub-rotina Minimum-Exhausting, descrita em Ris e colegas, algoritmo 3 [RBMJ10]. Minimum-Exhausting realiza uma busca em profundidade no reticulado Booleano. A sub-rotina inicia adicionando em uma pilha um elemento que tem custo mínimo em uma dada cadeia do espaço de busca. No início de cada iteração, é selecionado o elemento $T$ que está no topo da pilha e são avaliados todos os elementos adjacentes a $T$ no grafo definido pelo diagrama de Hasse do reticulado, isto é, todos os elementos que possuem distância de Hamming para $T$ igual a um. Em seguida, é adicionado na pilha todo elemento $Y$ adjacente a $T$ tal que: (i) $Y$ não foi removido do espaço de busca pelas coleções de restrições; (ii) $Y$ tem um custo menor ou igual a $c(T)$; (iii) $Y$ não está na pilha. Se nenhum elemento adjacente a $T$ é adicionado na pilha, então $T$ é considerado um "mínimo esgotado", o que implica que $T$ é removido da pilha e incluído nas coleções de restrições inferiores e superiores, encerrando a iteração corrente. A sub-rotina itera até que a pilha se torne vazia.

Mostraremos que Minimum-Exhausting tem um erro que pode levar a soluções sub-ótimas. Nas figuras 4.1 e 4.2 exibimos um exemplo de uma instância $\langle S, c\rangle$ do problema U-curve na qual todos os elementos de custo mínimo do reticulado Booleano podem ser perdidos durante a execução da sub-rotina Minimum-Exhausting. Esta sub-rotina é chamada na figura 4.1(c), na qual a busca em profundidade inicia empilhando em uma pilha o elemento 11100. A busca ramifica até que o elemento 01110 seja empilhado (figura 4.1(d)); uma vez que 01110 não tem nenhum elemento fora da pilha, a sub-rotina desempilha 01110 e o adiciona nas coleções de restrições, e recua para o elemento 01111 (figura 4.2(a)). O elemento 01111 também não tem elementos adjacentes fora da pilha, desta forma 01111 é desempilhado e incluído nas coleções de restrições - tal inclusão remove 
do espaço de busca corrente o único mínimo global, 00001, perdendo-o (figura 4.2(b)).

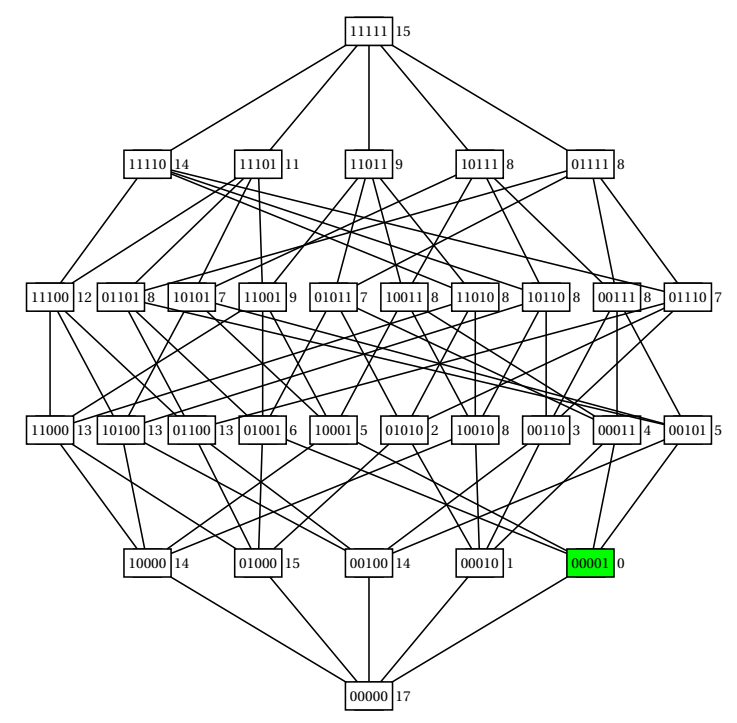

(a)

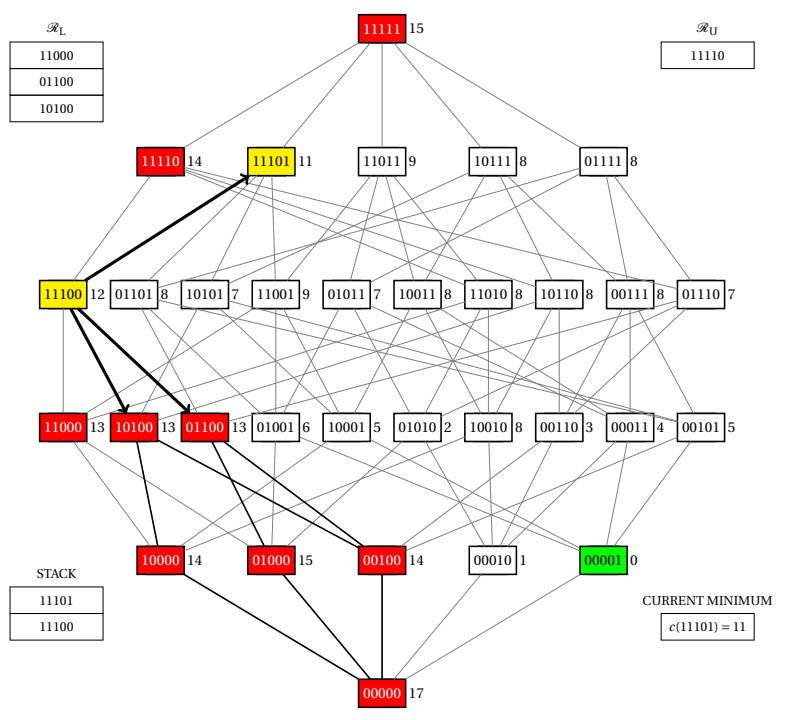

(c)

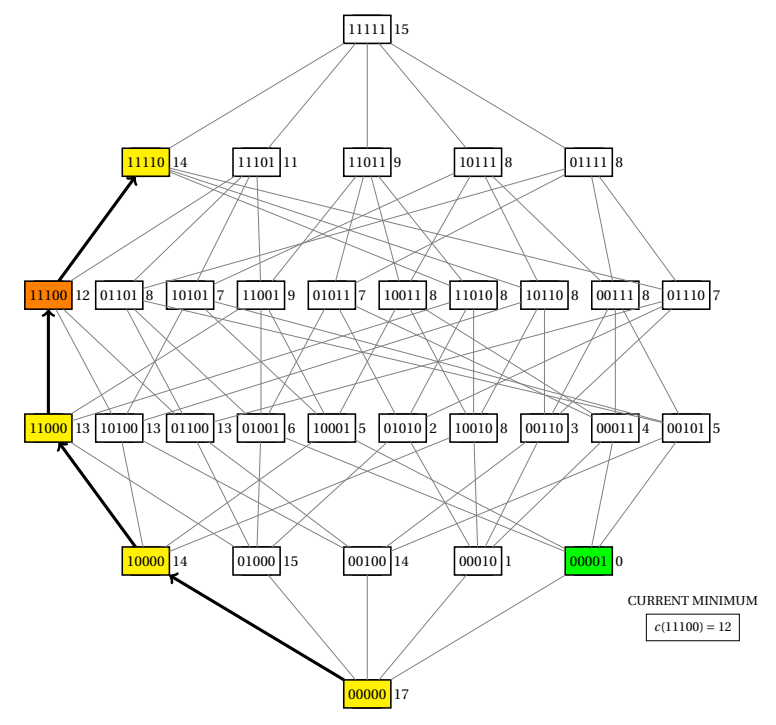

(b)
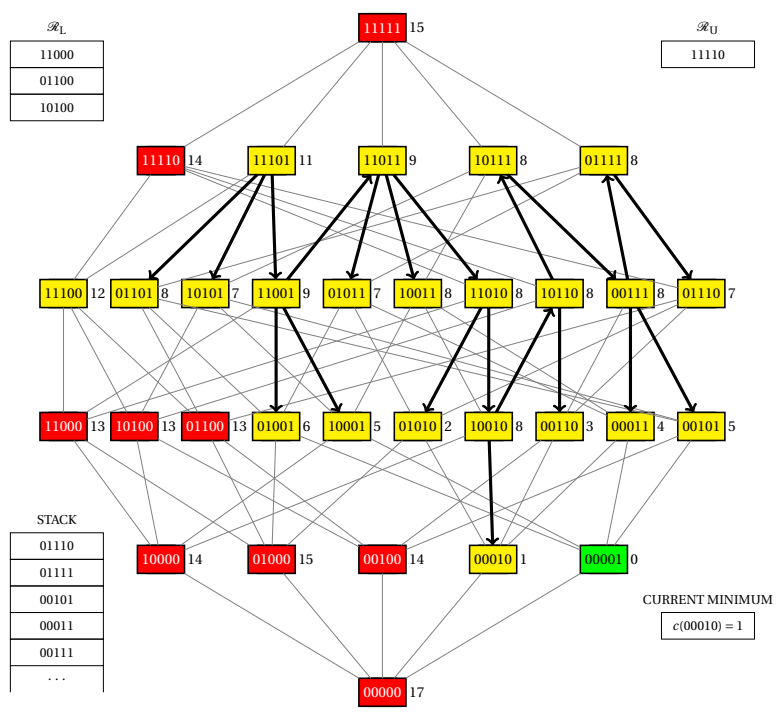

(d)

Figura 4.1: um contra-exemplo da correção da sub-rotina Minimum-Exhausted do algoritmo U-CURVE.

\subsection{Princípios de funcionamento do novo algoritmo}

Apresentaremos agora os princípios de funcionamento do novo algoritmo, o qual leva em consideração quatro condições suficientes para remover elementos não-visitados do espaço de busca sem o risco de perder mínimos globais. A seguir, enunciaremos as condições e demonstraremos que as mesmas de fato são suficientes.

Proposição 4.2.1. Sejam $\langle S, c\rangle$ uma instância do problema $U$-curve, $\mathcal{P}(S)$ o espaço de busca, $\mathcal{X} \subseteq \mathcal{P}(S)$ um espaço de busca corrente, e $X$ um elemento de $\mathcal{X}$. Se existe um elemento $Y \in \mathcal{X}$ tal que $Y \subseteq X$ e $c(Y)>c(X)$, então todos os elementos em $[\emptyset, Y]$ têm custo maior que $c(X)$.

Demonstração. Consideremos os elementos $X, Y \in \mathcal{P}(S)$ e $Z \in[\emptyset, Y]$ tais que $Y \subseteq X$ e $c(Y)>$ $c(X)$. Pela definição de $c$, vale que $c(Y) \leq \max \{c(Z), c(X)\}$. Logo, $c(Y) \leq c(Z)$ ou $c(Y) \leq c(X)$, e 


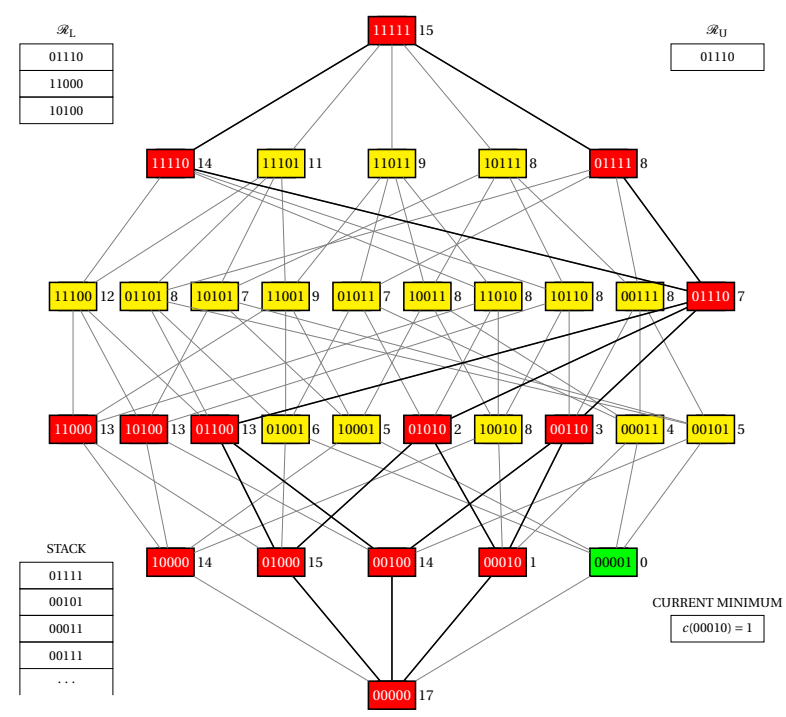

(a)

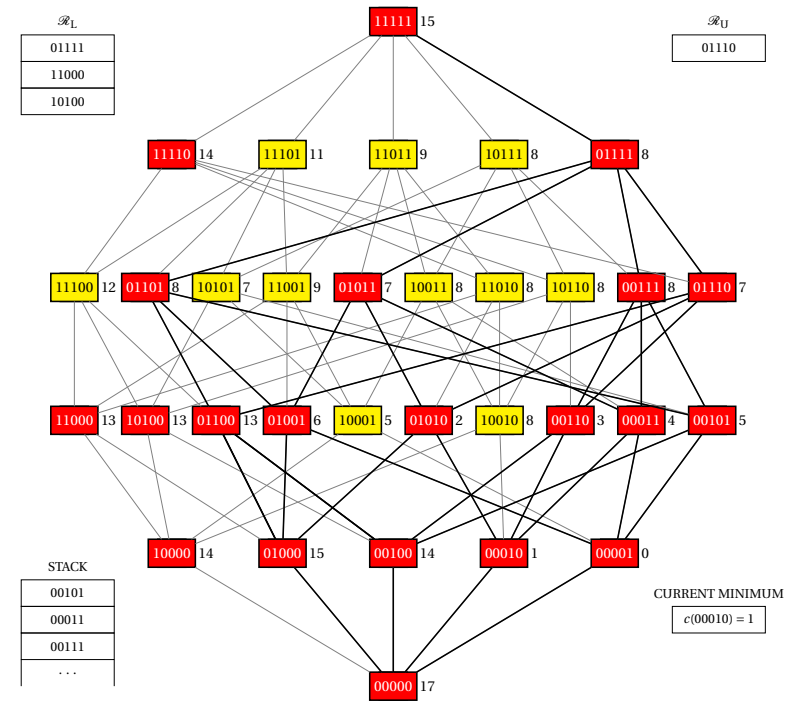

(b)

Figura 4.2: continuação do contra-exemplo da figura 4.1, que mostra uma simulação da sub-rotina Minimum-Exhausted que tem uma sequência de remoção de elementos que leva a perda de 00001, o único mínimo global do espaço de busca (figura 4.2(b)).

$c(Y)>c(X)$. Dessa forma, temos que $c(Z) \geq c(Y)>c(X)$, o que implica que todos os elementos em $[\emptyset, Y]$ têm custo maior que $c(X)$.

O resultado da proposição 4.2.1 também vale para o reticulado Booleano $(\mathcal{P}(S), \supseteq)$.

Proposição 4.2.2. Sejam $\langle S, c\rangle$ uma instância do problema U-curve, $\mathcal{P}(S)$ o espaço de busca, $\mathcal{X} \subseteq \mathcal{P}(S)$ um espaço de busca corrente, e $X$ um elemento de $\mathcal{X}$. Se existe um elemento $Y \in \mathcal{X}$ tal que $X \supseteq Y$ e $c(Y)>c(X)$, então todos os elementos em $[Y, S]$ têm custo maior que $c(X)$.

Demonstração. Aplicando o princípio da dualidade, o resultado da proposição 4.2.1 pode ser estendido para o reticulado Booleano $(\mathcal{P}(S), \supseteq)$.

Sejam $A$ e $B$ elementos de $\mathcal{P}(S)$. $A$ é adjacente inferior a $B$ (e $B$ é adjacente superior a $A)$ se $A \subseteq B$ e não existir um elemento $X \in \mathcal{P}(S)$ tal que $A \subset X \subset B$.

Há um resultado básico em Álgebra Booleana, mostrado a seguir, que também garante a remoção de um elemento do espaço de busca sem o risco de perder mínimos globais.

Proposição 4.2.3. Sejam $S$ um conjunto finito e não-vazio, $\mathcal{P}(S)$ o espaço de busca, $\mathcal{X} \subseteq \mathcal{P}(S)$ um espaço de busca corrente, e $X$ um elemento de $\mathcal{X}$. Se para cada elemento $Y \in \mathcal{P}(S)$ tal que $Y$ é adjacente inferior a $X,[\emptyset, Y] \cap \mathcal{X}=\emptyset$, então $[\emptyset, X]-\{X\}$ não contém um elemento de $\mathcal{X}$.

Demonstração. Consideremos um elemento $X \in \mathcal{X}$ e que, para cada elemento $Y \in \mathcal{P}(S)$ tal que $Y$ é adjacente inferior a $X,[\emptyset, Y] \cap \mathcal{X}=\emptyset$. Devido ao fato de que $X$ é o menor limite superior de seus elementos adjacentes inferiores, $\cup\{[\emptyset, Y]: Y$ é adjacente inferior a $X\}=[\emptyset, X]-X$. Portanto, $[\emptyset, X]-\{X\}$ não contém um elemento de $\mathcal{X}$.

O resultado da proposição 4.2.3 pode ser estendido para o reticulado Booleano $(\mathcal{P}(S), \supseteq)$.

Proposição 4.2.4. Sejam $S$ um conjunto finito e não-vazio, $\mathcal{P}(S)$ o espaço de busca, $\mathcal{X} \subseteq \mathcal{P}(S)$ um espaço de busca corrente, e $X$ um elemento de $\mathcal{X}$. Se para cada elemento $Y \in \mathcal{P}(S)$ tal que $Y$ é adjacente superior a $X,[Y, S] \cap \mathcal{X}=\emptyset$, então $[X, S]-\{X\}$ não contém um elemento de $\mathcal{X}$.

Demonstração. Aplicando o princípio da dualidade, o resultado da proposição 4.2.3 também vale para o reticulado Booleano $(\mathcal{P}(S), \supseteq)$. 
Visando corrigir o erro da sub-rotina Minimum-Exhausting, foi desenvolvido um novo algoritmo que não perde mínimos globais. O novo algoritmo trata os elementos que são visitados durante uma busca como vértices de um grafo. As arestas do grafo são definidas pela relação de adjacência dos elementos no reticulado Booleano $(\mathcal{P}(S), \subseteq)$. Cada vértice do grafo é armazenado em uma estrutura de dados chamada "nó"; um nó também tem uma "marca (flag) de restrição superior" e uma "marca de restrição inferior", que são variáveis Booleanas que assinalam se o vértice contido no nó foi removido do espaço de busca, respectivamente, pelas coleções de restrições inferiores e superiores. O algoritmo também leva em consideração as proposições 4.2.1, 4.2.2, 4.2.3 e 4.2.4 para a remover elementos do espaço de busca. Há dois tipos de remoção de elementos do espaço de busca:

- sejam $X$ e $Y$ elementos de $\mathcal{X} \subseteq \mathcal{P}(S)$ tais que $Y$ é adjacente inferior (no caso dual, superior) a $X$ e ambos são vértices do grafo. Se $c(Y)>c(X)$, então pela proposição 4.2.1 (4.2.2) o intervalo $[\emptyset, Y]([Y, S])$ deve ser removido do espaço de busca. Portanto, $Y$ é incluído na coleção de restrições inferiores (superiores) e seu respectivo nó é assinalado com a marca de restrição inferior (superior). Além disso, dado um vértice $Z$ do grafo, se $Z$ é contido propriamente em (contém propriamente) $Y$, então $Z$ é removido do grafo;

- seja $X$ um elemento de $\mathcal{X} \subseteq \mathcal{P}(S)$ que é um vértice do grafo. Se não existe elemento adjacente inferior (no caso dual, superior) a $X$ em $\mathcal{X}$, então pela proposição 4.2 .3 (4.2.4) o intervalo $[\emptyset, X]([X, S])$ deve ser removido do espaço de busca; portanto, $X$ é tratado como o elemento $Y$ do item anterior.

Nas figuras 4.3-4.9 mostramos uma simulação da execução do novo algoritmo para abordar o problema U-curve. Este algoritmo é simulado da seguinte maneira:

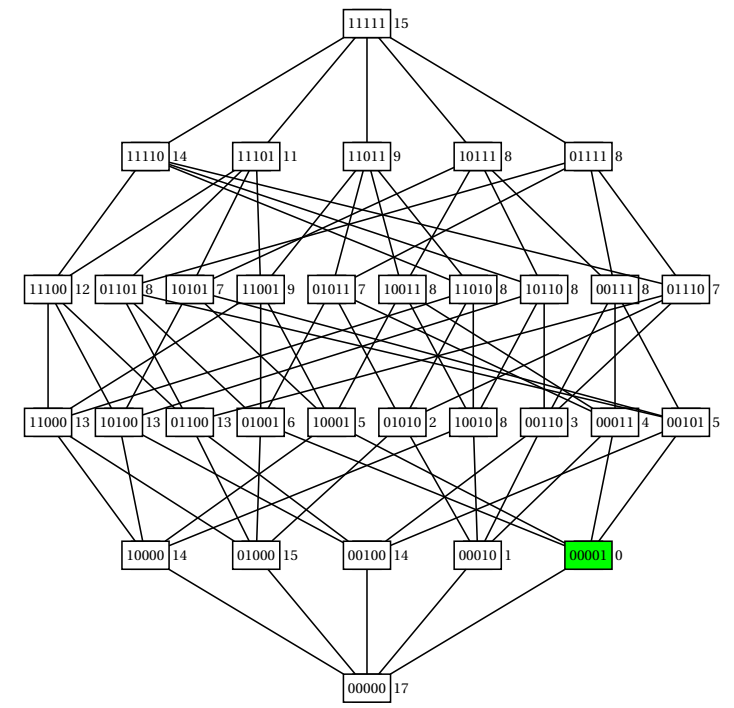

(a)

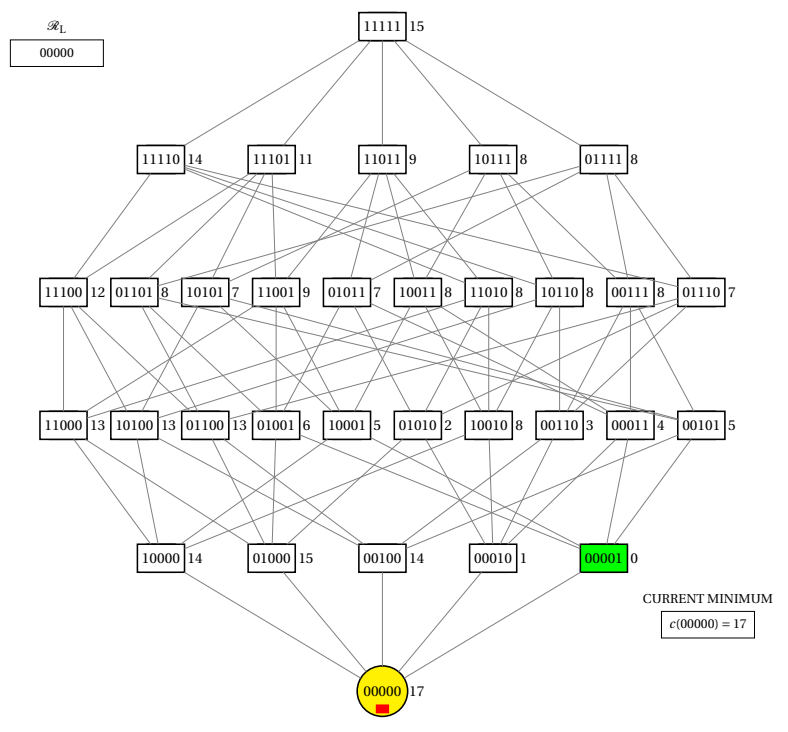

(b)

Figura 4.3: uma simulação do novo algoritmo para abordar o problema U-curve.

- Figura 4.3(a): uma instância com cinco elementos e função custo $c$, esta última definida pelos números ao lado dos nós; o elemento 00001 (em verde) tem custo mínimo;

- Figura 4.3(b): o algoritmo adiciona no grafo o elemento 00000, que é minimal no espaço de busca, isto é, não há um outro elemento no espaço de busca que seja contido propriamente em 00000. Uma vez que 00000 é minimal, ele também é incluído na coleção de restrições inferiores e é assinalado com a marca de restrição inferior; 


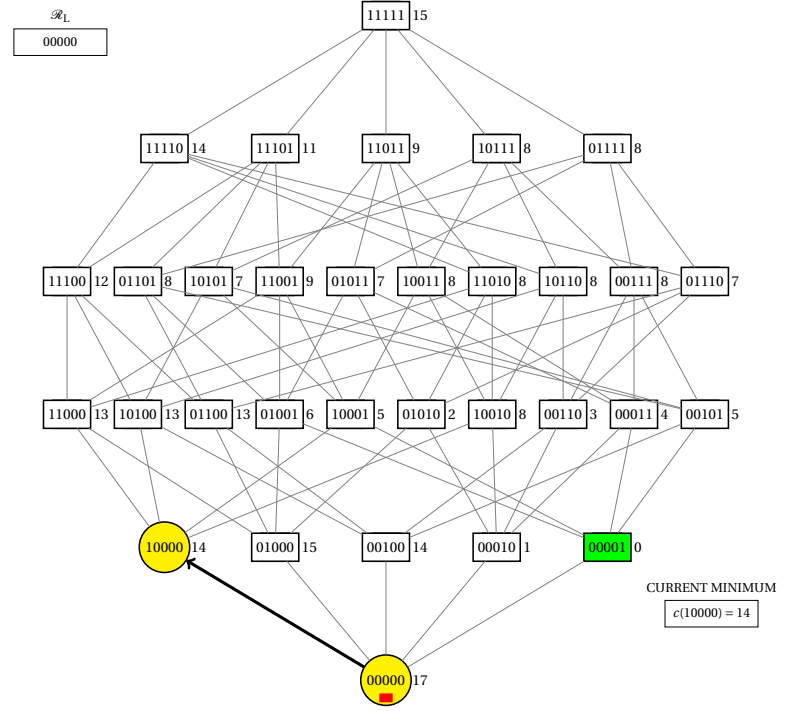

(a)

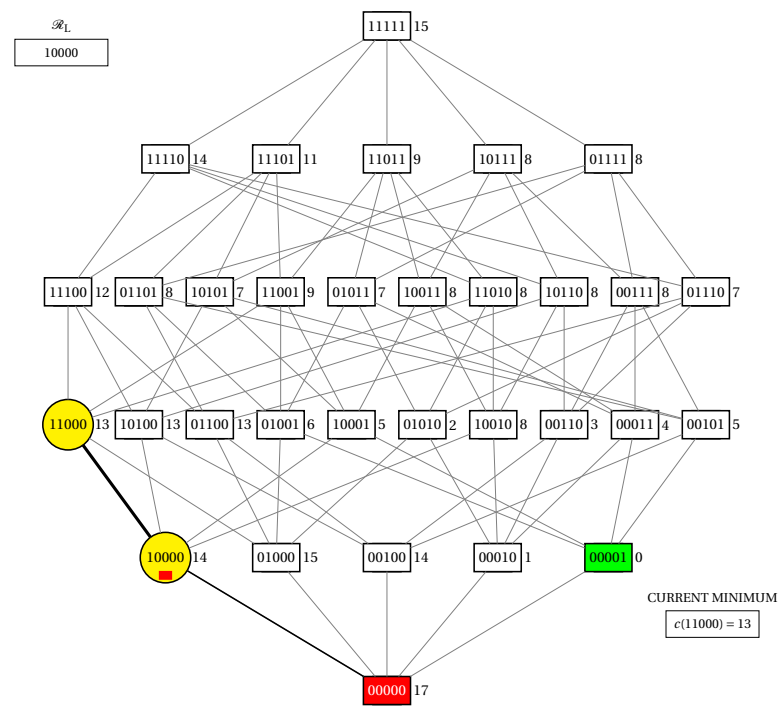

(c)

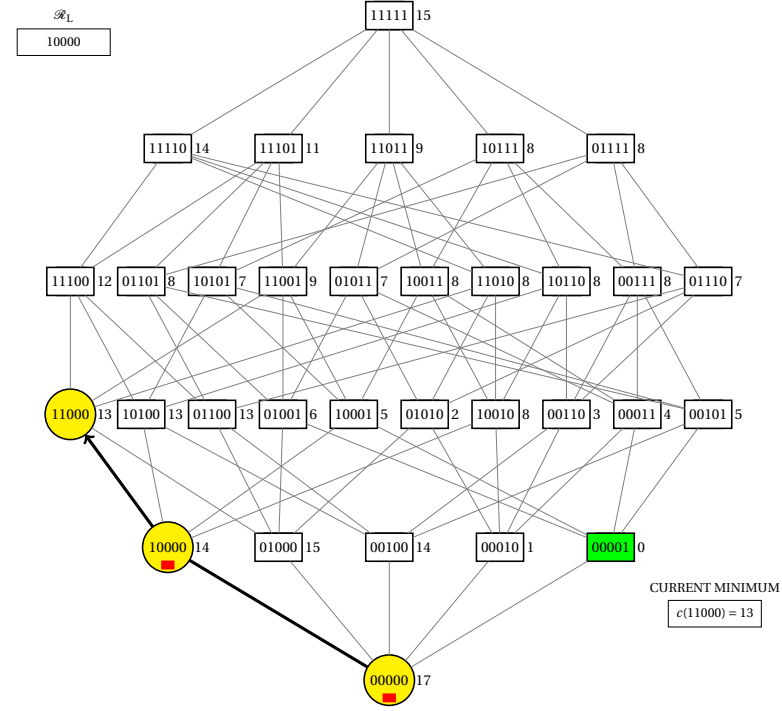

(b)

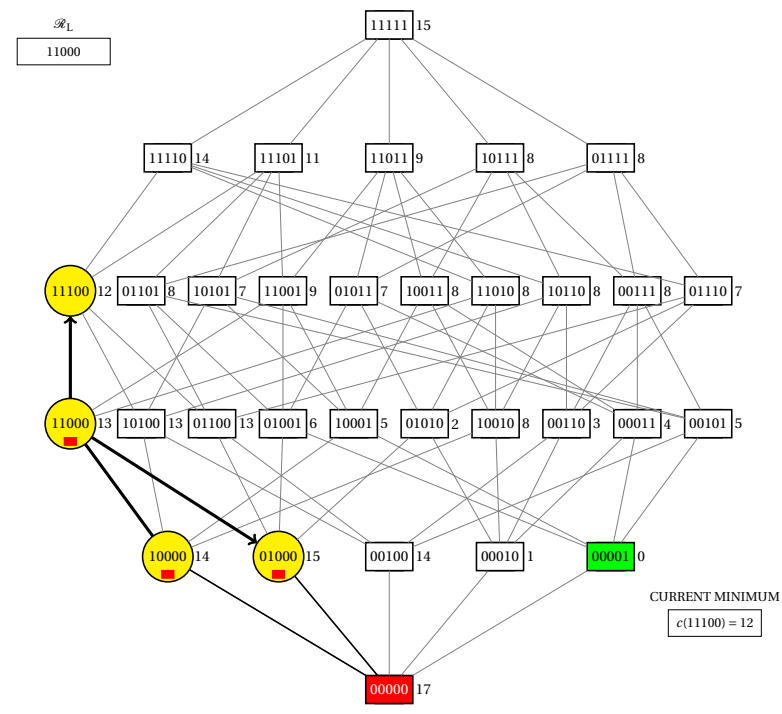

(d)

Figura 4.4: continuação da simulação iniciada na figura 4.3.

- Figura 4.4(a): 10000, adjacente a 00000, é visitado. $c(10000) \leq c(00000)$, portanto 10000 é adicionado no grafo;

- Figura 4.4(b): é visitado o elemento 11000, adjacente a 10000. $c(11000)<c(10000)$, portanto 11000 é adicionado no grafo. Além disso, devido à proposição 4.2.1, 10000 é assinalado com a marca de restrição inferior e incluído na coleção de restrições inferiores;

- Figura 4.4(c): 00000 é contido propriamente em um elemento da coleção de restrições inferiores (10000), portanto ele é removido do grafo;

- Figura 4.4(d): 01000, adjacente a 11000, é visitado; uma vez que $c(01000)>c(11000), 01000$ é incluído na coleção de restrições inferiores e assinalado com a marca de restrição inferior; 11100, adjacente a 11000, é visitado; $c(11000)>c(11100)$, portanto 11000 é incluído na coleção de restrições inferiores e assinalado com a marca de restrição inferior; 


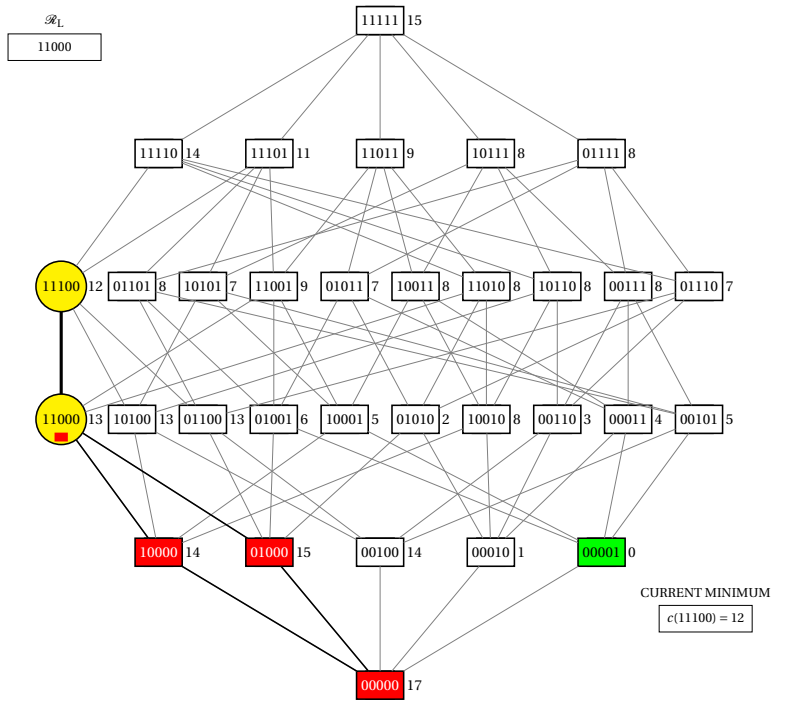

(a)

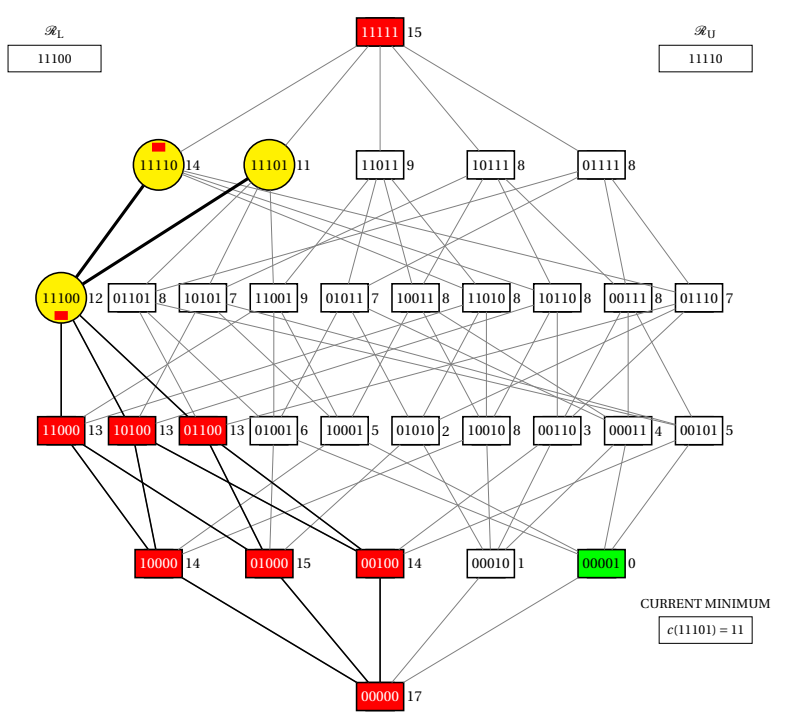

(c)

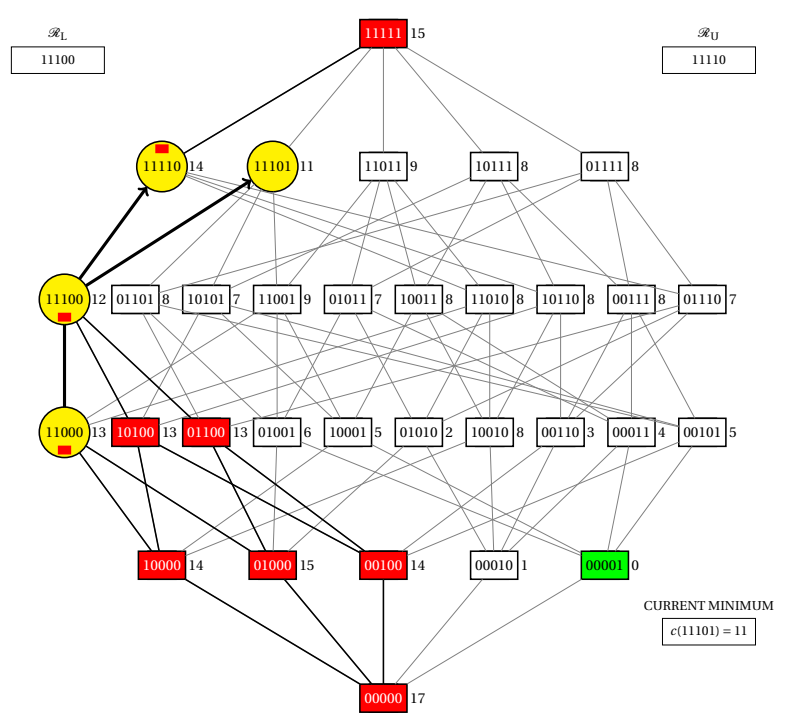

(b)

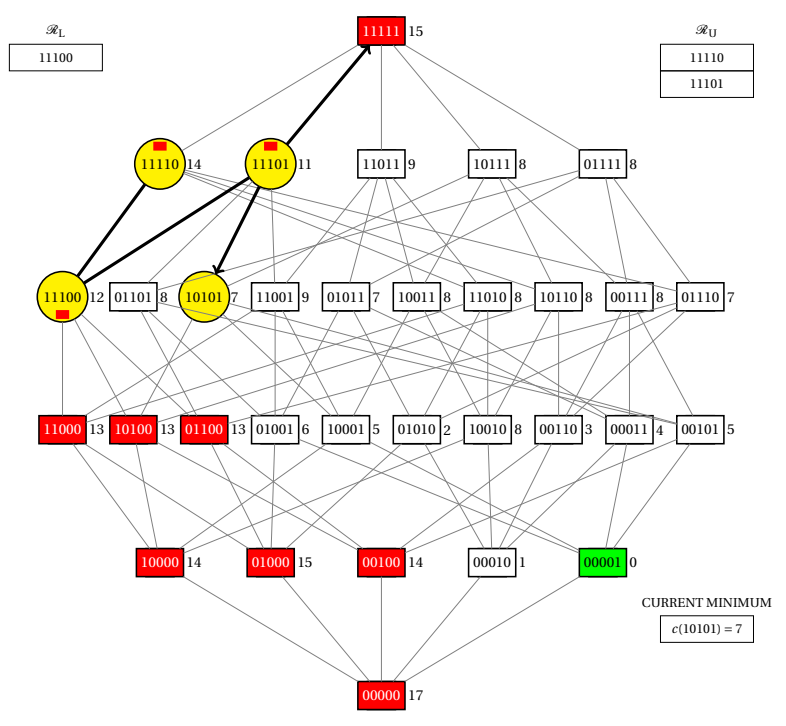

(d)

Figura 4.5: continuação da simulação da figura 4.4.

- Figura 4.5(a): 10000 e 01000 estão incluídos propriamente em um elemento da coleção de restrições inferiores (11000), portanto eles são removidos do grafo;

- Figura 4.5(b): 11110, adjacente a 11100, é visitado; uma vez que $c(11110)>c(11100), 11110$ é incluído na coleção de restrições superiores e assinalado com a marca de restrição superior; 11101, adjacente a 11100, é visitado; $c(11100)>c(11101)$, portanto 11100 é incluído na coleção de restrições inferiores e assinalado com a marca de restrição inferior;

- Figura 4.5(c): 11000 é contido propriamente em um elemento da coleção de restrições inferiores (11100), portanto ele é removido do grafo;

- Figura 4.5(d): é verificado que 11111, adjacente a 11101, não pertence ao espaço de busca; logo, um outro elemento é verificado. O elemento 10101, adjacente a 11101, é visitado; uma vez que $c(10101)<c(11101), 10101$ é adicionado no grafo e 11101 é incluído na coleção de restrições superiores e assinalado com a marca de restrição superior; 


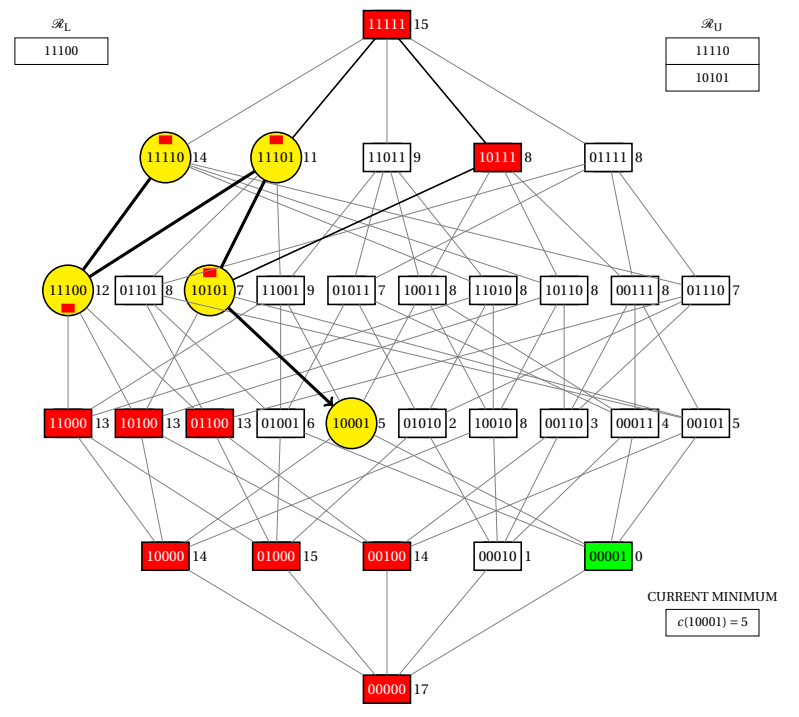

(a)

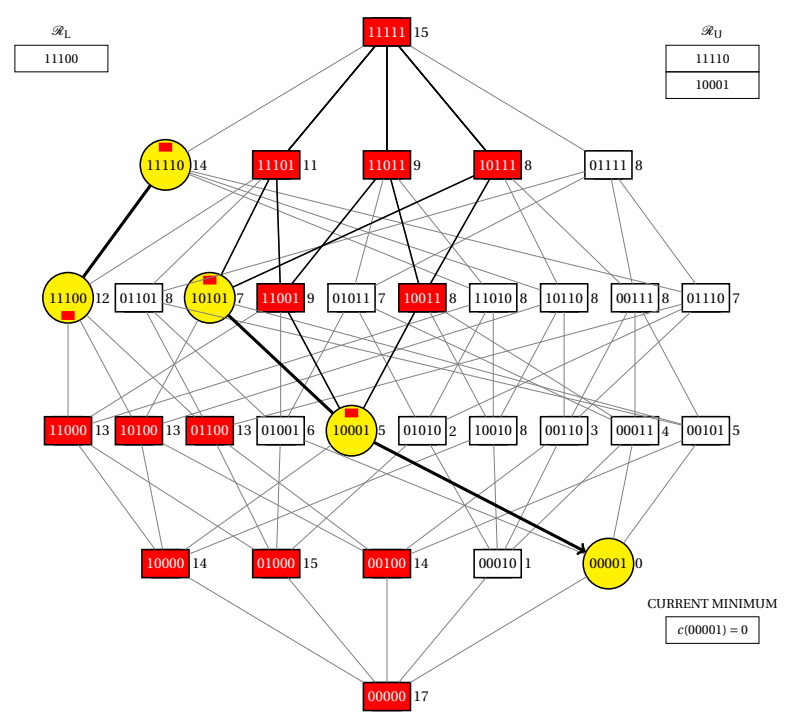

(c)

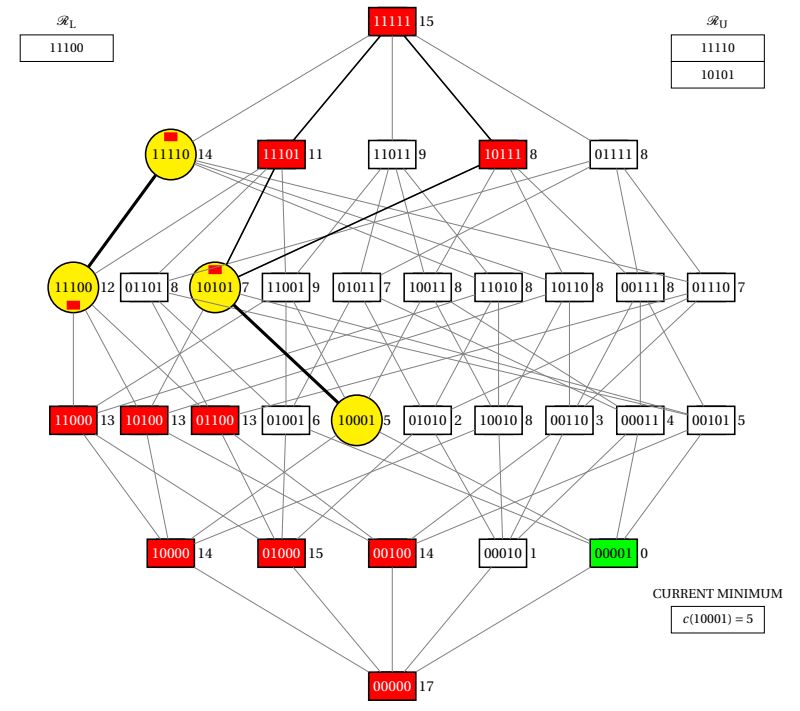

(b)

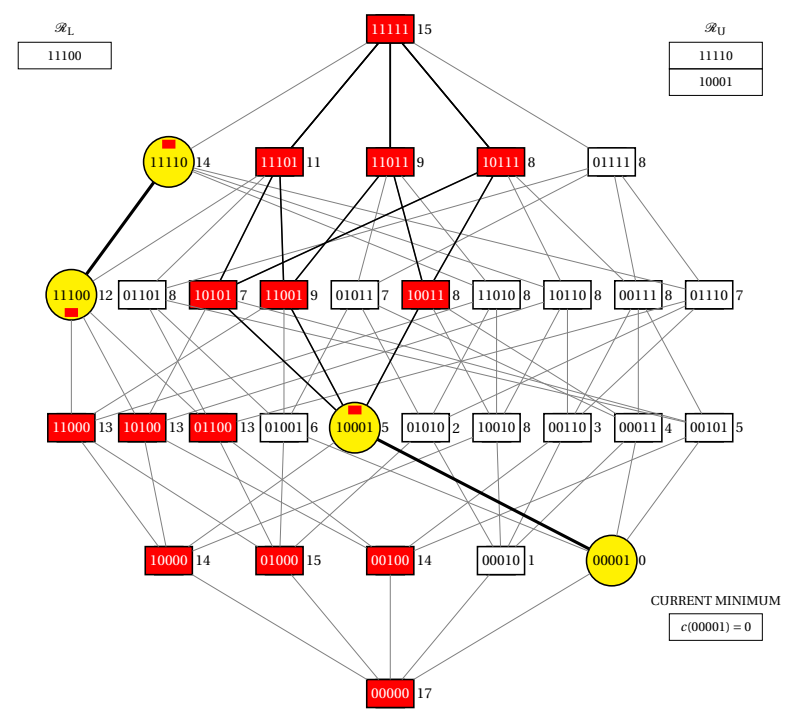

$(\mathrm{d})$

Figura 4.6: continuação da simulação da figura 4.5.

- Figura 4.6(a): 10001, adjacente a 10101, é visitado; uma vez que $c(10101)>c(10001), 10001$ é adicionado no grafo e 10101 é incluído na coleção de restrições superiores e assinalado com a marca de restrição superior;

- Figura 4.6(b): 11101 contém propriamente em um elemento da coleção de restrições superiores (10101), portanto ele é removido do grafo;

- Figura 4.6(c): é visitado o elemento 00001, adjacente a 10001. Uma vez que $c(10001)>$ $c(00001), 10001$ é incluído na coleção de restrições superiores e assinalado com a marca de restrição superior;

- Figura 4.6(d): 10101 é contido propriamente em um elemento da coleção de restrições superiores (10101), portanto ele é removido do grafo; 


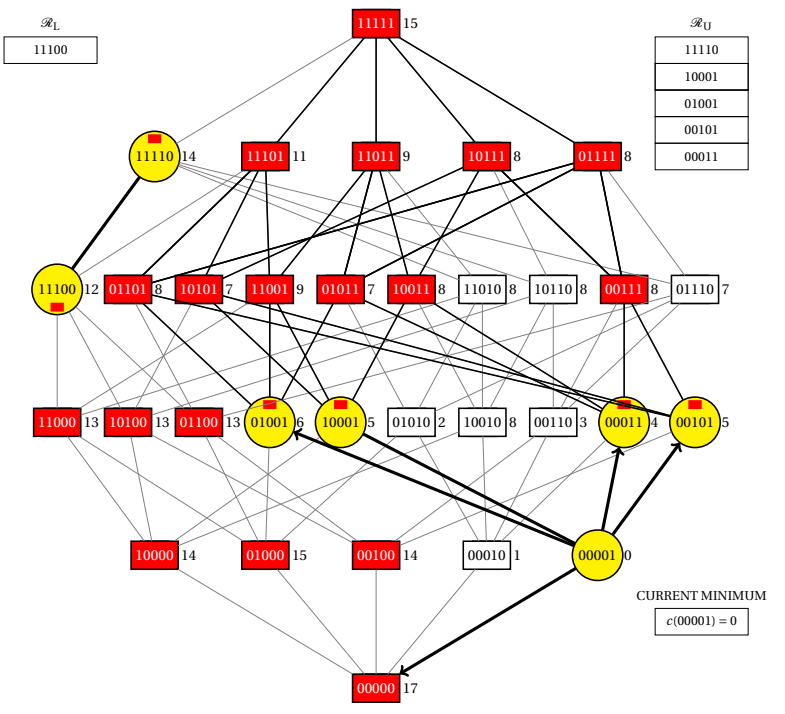

(a)

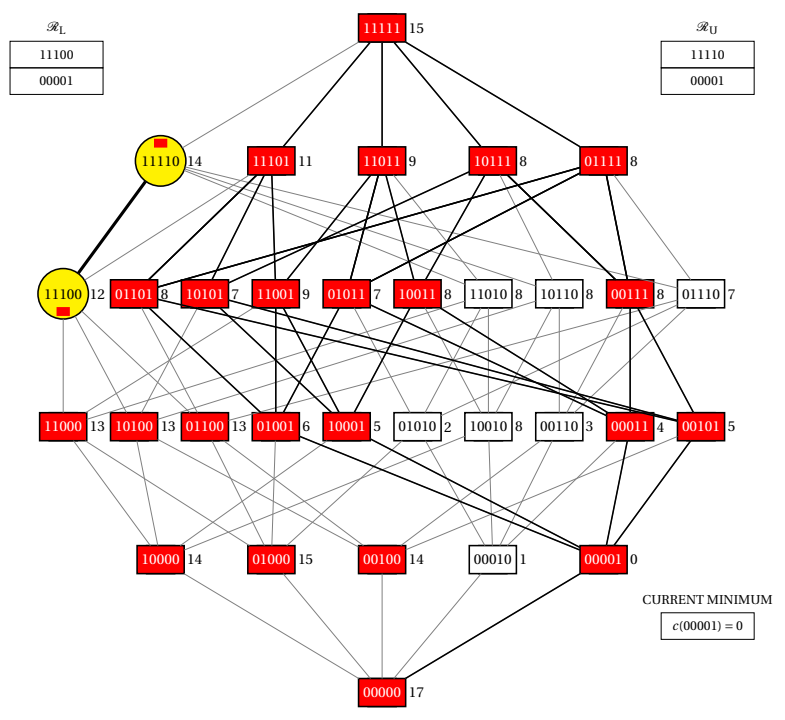

(c)

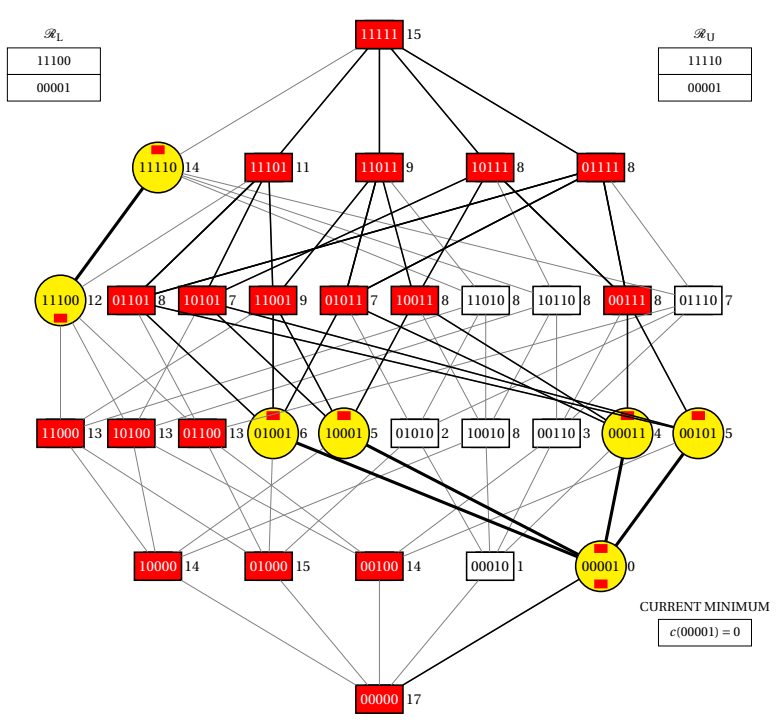

(b)

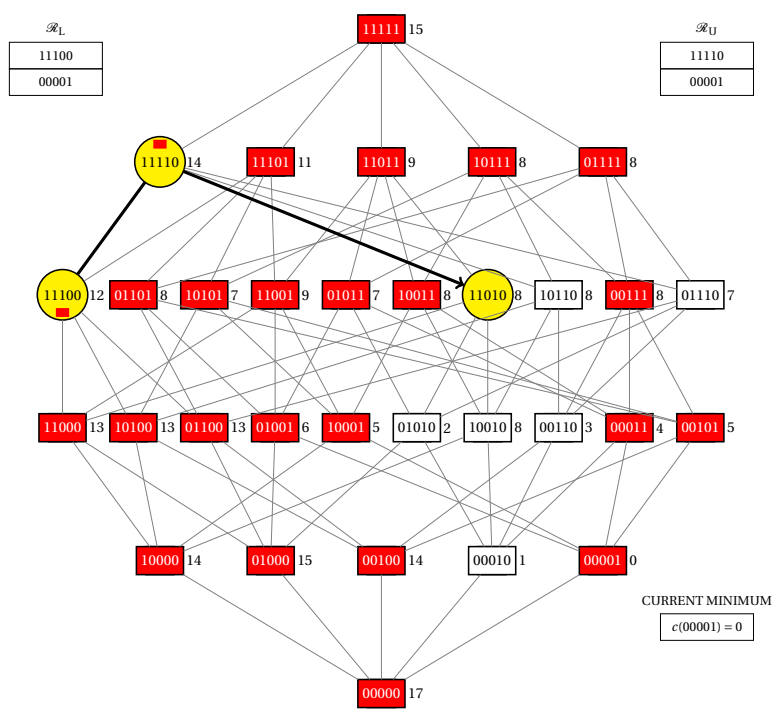

(d)

Figura 4.7: continuação da simulação da figura 4.6.

- Figura 4.7(a): é verificado que 00000, adjacente a 00001, não pertence ao espaço de busca, logo um outro elemento é verificado. São visitados todos os elementos adjacente superiores a 00001, 01001, 00101 e 00011. Todos eles têm custo maior que $c(00001)$ e são incluídos na coleção de restrições superiores e assinalados com a marca de restrição superior;

- Figura 4.7(b): uma vez que 00001 não tem elementos adjacentes inferiores (superiores) no espaço de busca, pela proposição 4.2.3 (4.2.4) 00001 é assinalado com a marca de restrição inferior (superior) e incluído na coleção de restrições inferiores (superiores);

- Figura 4.7(c): são removidos do grafo todos os vértices que estão contidos propriamente (contêm propriamente) 00001, Além disso, 00001 não pertence ao espaço de busca e não tem elemento adjacente no espaço de busca, portanto ele também é removido do grafo;

- Figura 4.7(d): é visitado o elemento 11010, adjacente a 11110. Uma vez que $c(11010) \leq$ $c(11110), 11010$ é adicionado no grafo; 


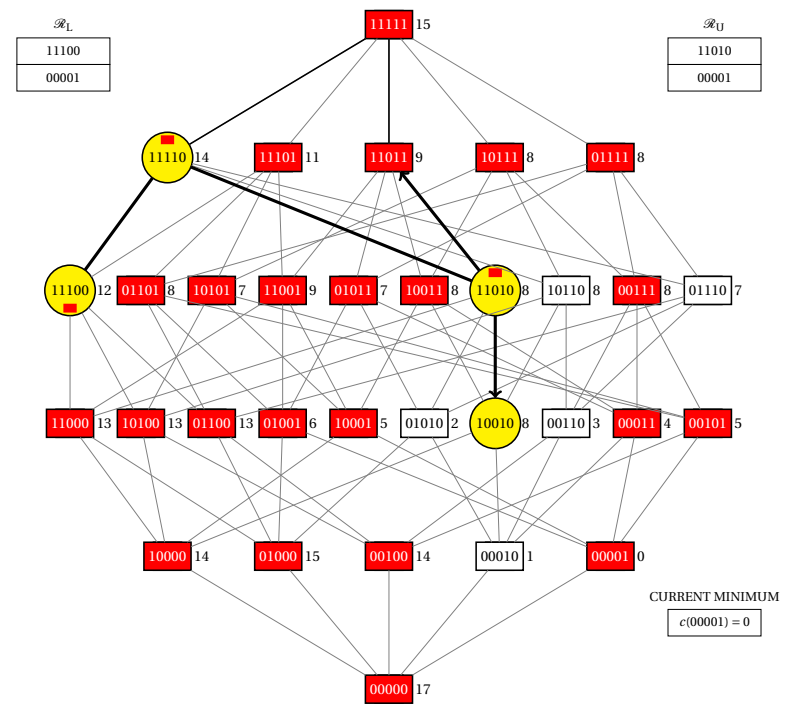

(a)

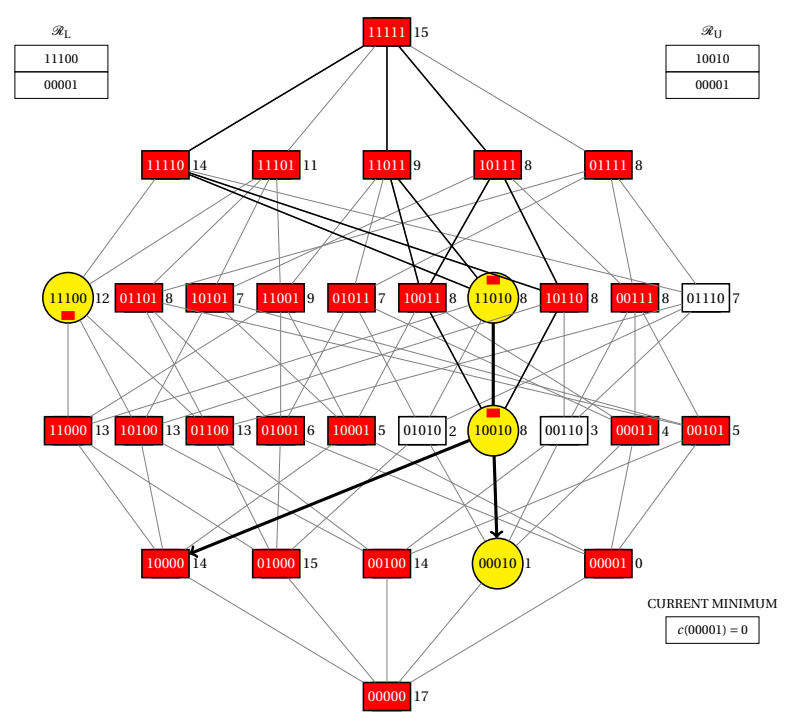

(c)

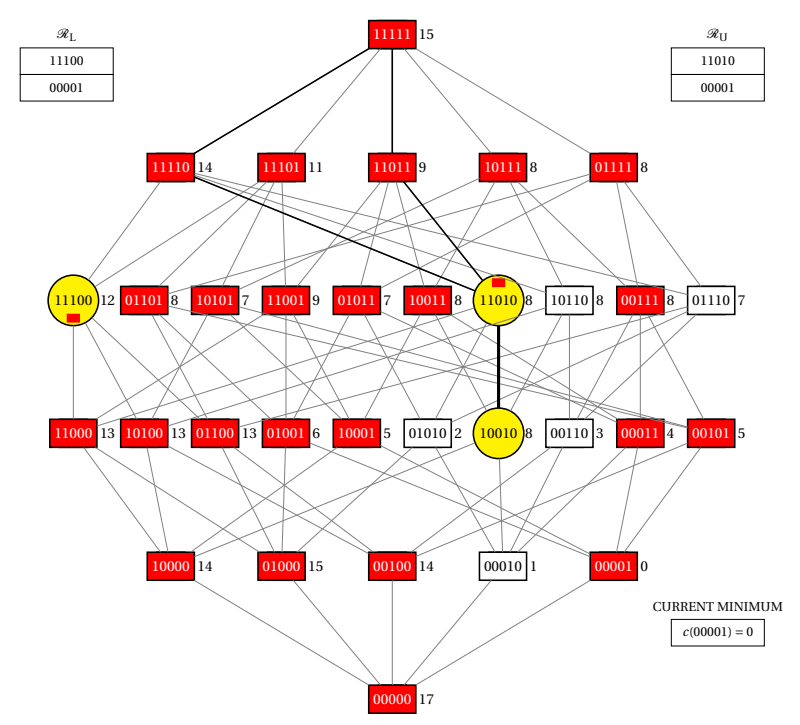

(b)

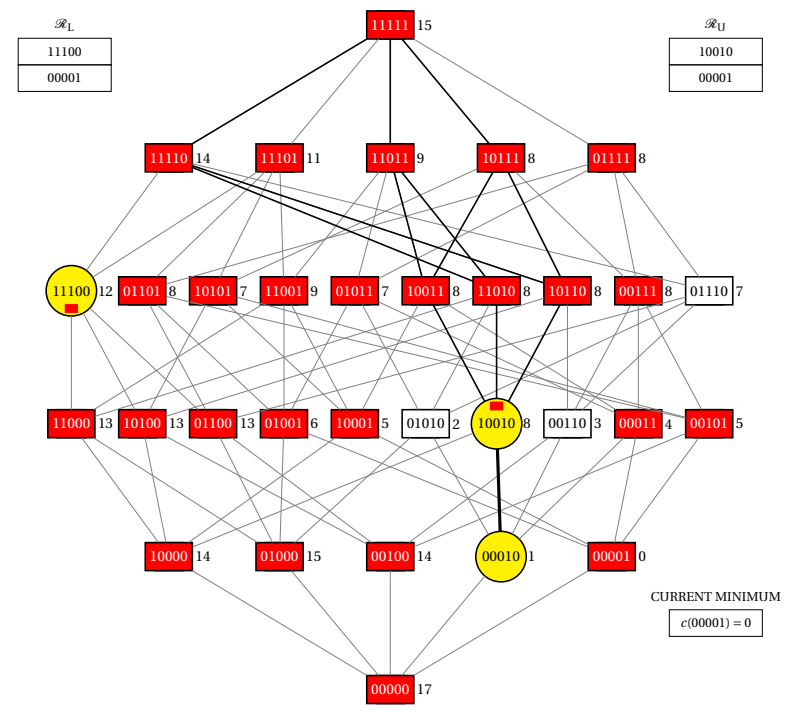

$(\mathrm{d})$

Figura 4.8: continuação da simulação da figura 4.\%.

- Figura 4.8(a): é verificado que 11011, adjacente a 11010, não pertence ao espaço de busca; como o outro adjacente superior a 11010 (11110) também não pertence, pela proposição 4.2.4, 11010 é incluído na coleção de restrições superiores e assinalado com a marca de restrição superior. É visitado 10010, adjacente a 110010; como $c(10010) \leq c(11010), 10010$ é adicionado no grafo;

- Figura 4.8(b): 11110 é contido propriamente em um elemento da coleção de restrições superiores (11010), portanto o mesmo é removido do grafo.

- Figura 4.8(c): é verificado se 10000, adjacente a 10010, pertence ao espaço de busca; uma vez que 10000 não pertence ao espaço de busca, um outro elemento é verificado. Em seguida, é visitado o elemento 00010, adjacente a 10010; uma vez que $c(10010)>c(00010), 10010$ é incluído na coleção de restrições superiores e assinalado com a marca de restrição superior;

- Figura 4.8(d): 11010 é contido propriamente em um elemento da coleção de restrições superiores (10010), portanto 11010 é removido do grafo; 


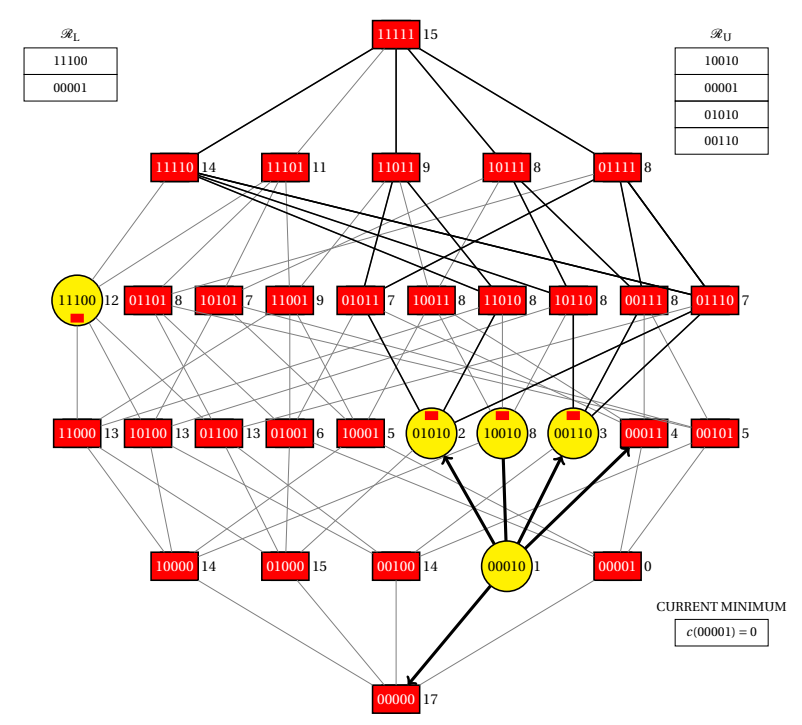

(a)

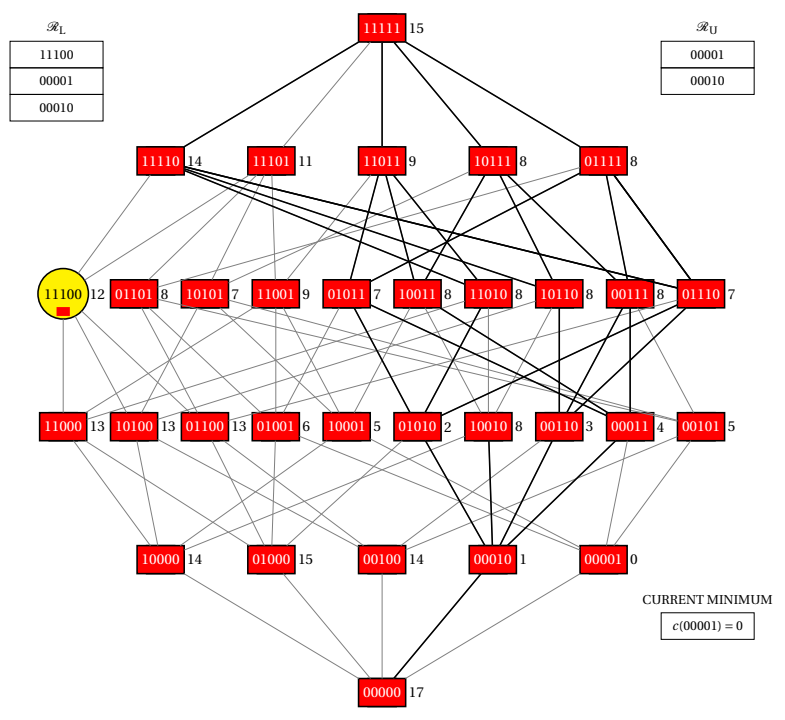

(c)

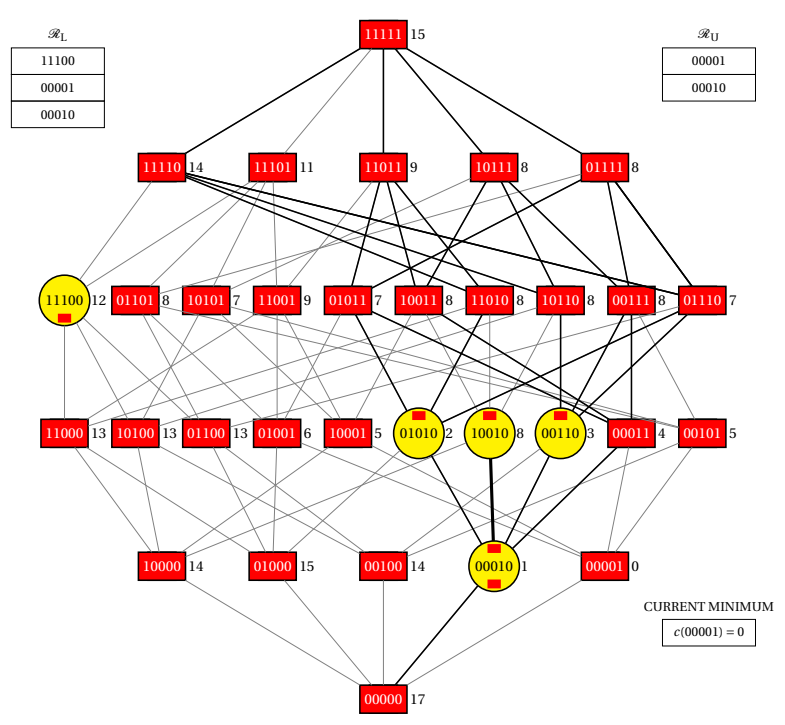

(b)

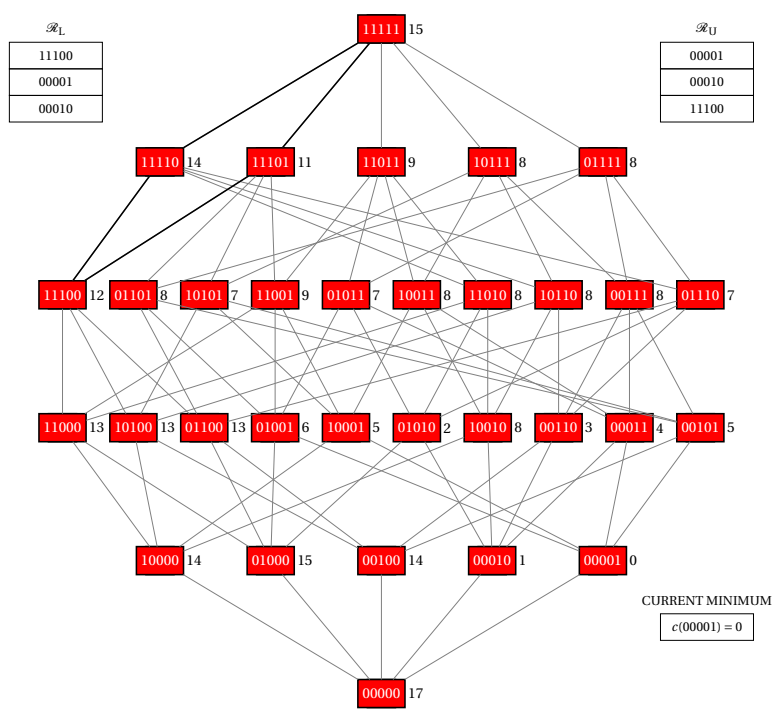

(d)

Figura 4.9: continuação da simulação da figura 4.8.

- Figura 4.9(a): são visitados todos os elementos adjacentes superiores a 00010 que não foram visitados ainda, mais especificamente 01010 e 00110; ambos têm custo maior que $c(00010)$ e são incluídos na coleção de restrições superiores e assinalados com a marca de restrição superior;

- Figura 4.9(b): uma vez que 00010 não tem elemento adjacente inferior (superior) no espaço de busca, pela proposição 4.2.3 (4.2.4) 00010 é assinalado com a marca de restrição inferior (superior) e incluído na coleção de restrições inferiores (superiores);

- Figura 4.9(c): são removidos do grafo todos os vértices que são propriamente contidos em (contêm propriamente) 00010. Além disso, 00010 não pertence ao espaço de busca e não tem elemento adjacente no espaço de busca, portanto ele é removido do grafo;

- Figura 4.9(d): 11100 não pertence ao espaço de busca e não tem elemento adjacente no mesmo. Portanto, 11100 é removido do grafo e incluído na coleção de restrições superiores. 
Apresentaremos agora o pseudo-código das sub-rotinas que implementam o algoritmo descrito nesta seção, assim como suas respectivas análises de complexidade de tempo.

\subsection{Pseudo-código e complexidade de tempo}

Nesta seção, apresentaremos o pseudo-código de U-CuRVE-SEARCH (UCS), um novo algoritmo para abordar o problema U-curve, que implementa os princípios de funcionamento introduzidos na seção 4.2. Mostraremos também uma análise de sua complexidade de tempo.

Daqui em diante, suporemos que qualquer coleção $\mathcal{R} \subseteq \mathcal{P}(S)$ é implementada utilizando uma lista duplamente encadeada. Portanto, a complexidade de uma busca em uma coleção é $O(n|\mathcal{R}|)$ (no qual $n$ é a cardinalidade do conjunto $S$ ), de uma inserção é $O(1)$ e de uma remoção é $O(1)$.

\subsubsection{Atualização das restrições inferiores e superiores}

Seja $X$ um elemento de $\mathcal{P}(S)$. $X$ é coberto por uma coleção de restrições inferiores (na forma dual, superiores) $\mathcal{R}_{L}\left(\mathcal{R}_{U}\right)$ se existe um elemento $R \in \mathcal{R}_{L}\left(R \in \mathcal{R}_{U}\right)$ tal que $X \subseteq R(R \subseteq X)$.

Descrição de algoritmo. UPDATE-LOWER-RESTRICTION recebe um elemento $X \in \mathcal{P}(S)$ e uma coleção de restrições inferiores $\mathcal{R}_{L}$. Se $X$ não é coberto por $\mathcal{R}_{L}$, então este algoritmo adiciona $X$ em $\mathcal{R}_{L}$ e remove de $\mathcal{R}_{L}$ todos os elementos que são contidos propriamente em $X$. Por fim, este algoritmo devolve a coleção atualizada $\mathcal{R}_{L}$.

Durante a simulação mostrada na figura 4.3, esta sub-rotina é equivalente às atualizações da coleção de restrições inferiores apresentadas nas figuras 4.3(b) (com $X=00000$ ), 4.4(b) (10000), 4.4(d) (01000), 4.4(d) (11000), 4.5(b) (11100), 4.7(b) (00001) e 4.9(b) (00010).

Análise de complexidade de tempo. Uma vez que $\mathcal{R}_{L}$ é implementada através de uma lista duplamente encadeada, o algoritmo consome $\mathrm{O}\left(n\left|\mathcal{R}_{L}\right|\right)$ unidades de tempo. A proposição a seguir nos fornece um limite mais preciso para o pior caso.

Proposição 4.3.1. Seja $\mathcal{R}_{L}$ uma coleção de restrições inferiores. Se não há elementos $A$ e $B$ em $\mathcal{R}_{L}$ tais que $A$ contenha propriamente $B$, então o tempo computacional exigido pelo algoritmo UPDATE-LOWER-RESTRICTION no pior caso é $\Theta\left(n 2^{n}\right)$.

Demonstração. Considere que $\mathcal{R}_{L}$ é a coleção de todos os subconjuntos de $S$ de cardinalidade $\lfloor n / 2\rfloor$, isto é, uma coleção com elementos de cardinalidade $\lfloor n / 2\rfloor$ tal que não há elemento que seja propriamente contido em um outro. Neste caso, a coleção de restrições tem $\left(\begin{array}{c}n \\ \lfloor n / 2\rfloor\end{array}\right)=\Omega\left(2^{n}\right)$ elementos. Uma vez que o reticulado Booleano de grau $n$ tem exatamente $2^{n}$ elementos, $O\left(2^{n}\right)$ é um limite superior para o número de elementos da coleção de restrições. Portanto, $\mathcal{R}_{L}$ tem $\Theta\left(2^{n}\right)$ elementos, o que implica que o tempo computacional exigido pelo algoritmo UPDATE-LOWER-RESTRICTION no pior caso é $\Theta\left(n 2^{n}\right)$.

Segue da proposição 4.3.1 que a remoção de elementos de $\mathcal{R}_{L}$ que são propriamente contidos em $X$ não muda o tempo assintótico exigido por esta sub-rotina. Todavia, na prática, a remoção de elementos cobertos leva a um melhor desempenho do algoritmo U-CuRVE-SEARCH, haja vista que a operação de leitura da coleção $\mathcal{R}_{L}$ é mais comum do que a operação de atualização da mesma.

Algoritmo dual. O algoritmo Update-Upper-Restriction recebe um elemento $X$ de $\mathcal{P}(S)$ e uma coleção de restrições superiores $\mathcal{R}_{U}$. Se $X$ não é coberto por $\mathcal{R}_{U}$, então este algoritmo adiciona $X$ em $\mathcal{R}_{U}$ e remove todos os elementos de $\mathcal{R}_{U}$ que contêm propriamente $X$. Por fim, este algoritmo devolve a coleção atualizada $\mathcal{R}_{U}$. 


\subsubsection{Elementos minimais e maximais}

Seja $X$ um elemento de $\mathcal{A} \subseteq \mathcal{P}(S)$. $X$ é minimal (na forma dual, maximal) em $\mathcal{A}$ se para qualquer $Y \in \mathcal{A}, Y \subseteq X(X \subseteq Y)$ implica que $X=Y$.

Agora seja $\mathcal{X}$ uma função que leva valores de $\mathcal{P}(S) \times \mathcal{P}(S)$ para $\mathcal{P}(S)$, definida como

$$
\mathcal{X}\left(\mathcal{R}_{L}, \mathcal{R}_{U}\right)=\mathcal{P}(S)-\bigcup\left\{[\emptyset, R]: R \in \mathcal{R}_{L}\right\}-\bigcup\left\{[R, S]: R \in \mathcal{R}_{U}\right\} .
$$

Dizemos que $\mathcal{X}\left(\mathcal{R}_{L}, \mathcal{R}_{U}\right)$ é um espaço de busca corrente do espaço de busca $\mathcal{P}(S)$.

Descrição de algoritmo. Minimal-Element recebe um conjunto finito e não-vazio $S$ e uma coleção de restrições inferiores $\mathcal{R}_{L}$. Se o espaço de busca corrente $\mathcal{X}\left(\mathcal{R}_{L}, \emptyset\right)$ é não vazio, então este algoritmo devolve um elemento $X$ que é minimal em $\mathcal{X}\left(\mathcal{R}_{L}, \emptyset\right)$; caso contrário, ele devolve $N I L$.

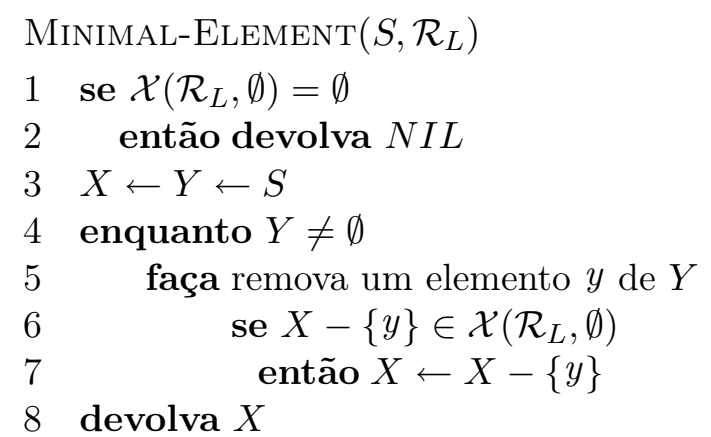

Durante a simulação mostrada na figura 4.3, esta sub-rotina é equivalente a seleção do elemento 00000 minimal em $\mathcal{X}(\emptyset, \emptyset)$, a qual é apresentada na figura 4.3(b).

Análise de complexidade de tempo. O laço das linhas 4-7 itera exatamente $n+1$ vezes e, em cada iteração, verificar se $X$ está em $\mathcal{X}\left(\mathcal{R}_{L}, \emptyset\right)$ consome $\mathrm{O}\left(n\left|\mathcal{R}_{L}\right|\right)$. Portanto, Minimal-Element consome $\mathrm{O}\left(n^{2}\left|\mathcal{R}_{L}\right|\right)$ unidades de tempo.

Algoritmo dual. MAXimal-Element recebe um conjunto finito e não-vazio $S$ e uma coleção de restrições superiores $\mathcal{R}_{U}$. Se o espaço de busca corrente $\mathcal{X}\left(\emptyset, \mathcal{R}_{U}\right)$ é não vazio, então este algoritmo devolve um elemento $X$ que é maximal em $\mathcal{X}\left(\emptyset, \mathcal{R}_{U}\right)$; caso contrário, ele devolve NIL.

\subsubsection{Busca em profundidade}

Seja $G$ um grafo. Ao longo deste capítulo, $G$ é um hipercubo de grau $|S|$, definido a partir do diagrama de Hasse do reticulado Booleano $(\mathcal{P}(S), \subseteq)$. Portanto, esta definição vale para o grafo apresentado na seção 4.2 .

Descreveremos agora uma estrutura de dados para implementar $G$. Além disso, tal estrutura de dados será utilizada para implementarmos o algoritmo proposto na seção 4.2. Um nó é uma estrutura contendo sete campos, dos quais os quatro primeiros mapeiam para um elemento de $\mathcal{P}(S)$, o quinto e o sexto mapeiam para uma coleção de nós e o último mapeia para um número real:

- "vertex" representa um vértice de $G$;

- "unverified" é utilizado pelo algoritmo de busca para controlar os elementos adjacentes a "vertex" no reticulado Booleano que já foram verificados ou não;

- "lower_adjacent" e "upper_adjacent" representam a topologia de G. Se eles estão vazios, então eles são equivalentes a, respectivamente, a "marca de restrição inferior" e a "marca de restrição superior" apresentadas na seção 4.2; 
- "lower_edges" e "upper_edges" representam as arestas que têm o vértice "vertex" como, respectivamente, o elemento adjacente inferior e superior no reticulado Booleano;

- "cost" armazena o custo de "vertex", o qual é computado utilizando-se a função custo $c$.

Sejam $\mathcal{G}$ uma coleção de nós, $\mathbf{Y}$ um nó de $\mathcal{G}$ e $\mathcal{X}\left(\mathcal{R}_{L}, \mathcal{R}_{U}\right)$ um espaço de busca corrente. $\mathbf{Y}[$ lower_adjacent $]$ (no caso dual, $\mathbf{Y}[$ upper_adjacent $])$ tem $\mathcal{P}(\mathbf{Y}[$ vertex $])(\mathcal{P}(S-\mathbf{Y}[$ vertex $]))$ como domínio. Para cada elemento y de $\mathbf{Y}[$ vertex $](S-\mathbf{Y}[$ vertex $]), y \notin \mathbf{Y}[$ lower_adjacent $]$ $(\mathbf{Y}[$ upper_adjacent $])$ implica que $\mathbf{Y}[$ vertex $]-\{y\}(\mathbf{Y}[$ vertex $] \cup\{y\})$ é coberto por $\mathcal{R}_{L}\left(\mathcal{R}_{U}\right)$.

Utilizando os objetos definidos até aqui, apresentaremos agora cinco regras que constituem o núcleo da dinâmica do algoritmo:

(i) Gerenciamento das marcas de restrição. Dado um elemento $X$ de $\mathcal{P}(S)$ tal que $X$ é adjacente inferior (no caso dual, superior) a $\mathbf{Y}[$ vertex $]$, se o único elemento em $\mathbf{Y}[$ vertex $]-X(X-$ $\mathbf{Y}[$ vertex $]$ ) não está em $\mathbf{Y}\left[\right.$ lower_adjacent] $(\mathbf{Y}[$ upper_adjacent $])$, então $\mathcal{R}_{L}\left(\mathcal{R}_{U}\right)$ cobre $X$.

Esta regra descreve uma implementação da "marca de restrição inferior" e da "marca de restrição superior" apresentadas na seção 4.2, de uma maneira que ela também armazena informação para verificação das condições de poda das proposições 4.2 .3 e 4.2.4;

(ii) Critério para parar uma busca. Se existe um elemento $X \in \mathcal{X}\left(\mathcal{R}_{L}, \mathcal{R}_{U}\right)$ adjacente a $\mathbf{Y}$ [vertex] tal que $X$ não é um vértice de $G$ (i.e., $\mathbf{X}[$ vertex $] \neq X$ para todo nó $\mathbf{X}$ de $\mathcal{G}$ ), então $X$ é um elemento adjacente não-visitado a $\mathbf{Y}[$ vertex];

(iii) Gerenciamento dos elementos adjacentes visitados. Se $X$ é um elemento adjacente não-visitado a $\mathbf{Y}$, então $\mathbf{Y}[$ unverified $]$ tem um elemento $y$ tal que $X=\mathbf{Y}[$ vertex $] \cup\{y\}$ ou $X=\mathbf{Y}[$ vertex $]-$ $\{y\}$.

Esta regra define uma maneira de se evitar que, para cada vez que é verificado se $\mathbf{Y}$ tem um elemento adjacente não-visitado, seja necessária a inspeção de todos os elementos adjacentes a $\mathbf{Y}[$ vertex $]$ em $\mathcal{P}(S)$;

(iv) Gerenciamento das arestas de $G$. Se $\mathbf{Y}[$ vertex $]$ é adjacente inferior (superior) a algum vértice $\mathbf{X}[$ vertex $]$ de $G$ e $G$ tem a aresta $\{\mathbf{X}[$ vertex $], \mathbf{Y}[$ vertex $]\}$, então a coleção $\mathbf{Y}[$ upper_edges $]$ $(\mathbf{Y}[$ lower_edges $])$ contém $\mathbf{X}$ e a coleção $\mathbf{X}[$ lower_edges $](\mathbf{X}[$ upper_edges $])$ contém $\mathbf{Y}$;

(v) Computação da função custo $c$. Toda vez que um nó $\mathbf{Y}$ é criado, o valor $c(\mathbf{Y}[$ vertex $])$ é computado e armazenado em $\mathbf{Y}[$ cost $]$. Dessa forma, toda vez que uma sub-rotina verifica $c(\mathbf{Y}[$ vertex $])$, ela de fato lê o valor correspondente em $\mathbf{Y}[\operatorname{cost}]$, o que consome $O(1)$ unidades de tempo.

A busca em profundidade é realizada em duas etapas: na primeira, dado um sub-grafo $G^{\prime}$ de $G$, que representa a topologia atual do espaço de busca, ela explora um vértice $X$ de $G^{\prime}$, verificando cada elemento $Y$ que é adjacente a $X$ e eventualmente definindo um sub-grafo $G^{\prime \prime}:=G^{\prime} \cup\{Y\}$; já na segunda, ela poda o sub-grafo $G^{\prime \prime}$, assim criando um novo sub-grafo $G^{\prime \prime \prime}$. Seja $G_{0}, \ldots, G_{k}$ uma sequência de sub-grafos, na qual $G_{0}$ é uma condição inicial e $G_{i}$ e $G_{i+1}, i=1,3,5, \ldots$, são, respectivamente, os resultados da primeira e da segunda etapas. O sub-grafo inicial $G_{0}$ é um grafo trivial, isto é, um grafo com um único vértice e nenhuma aresta. Na primeira iteração, $G_{1}$ é ou $G_{0}$ ou um $G_{0}$ aumentado (i.e., $G_{1}$ é $G_{0}$ um vértice e uma aresta extras), e $G_{2}$ é ou $G_{1}$ ou um $G_{1}$ podado (i.e., $G_{2}$ é $G_{1}$ sem alguns vértices e arestas). Em qualquer iteração $i, i>1$, esta dinâmica é repetida com $G_{2(i-1)}$ no papel de $G_{0}$.

Durante as iterações da busca, um sub-grafo $G_{k}$ pode ser um grafo desconexo. Além disso, $G_{k}$ pode ter uma topologia tal que uma poda não pode ser realizada apelas utilizando suas arestas. Na figura 4.13(a) é mostrado um exemplo deste tipo de poda. Uma vez que uma poda de um vértice $X$ não pode ser realizada utilizando somente as arestas de $G_{k}$, é necessário inspecionar todo vértice $Y$ de $G_{k}$, verificando a relação de ordem entre $X$ e $Y$. Portanto, a poda é feita em duas etapas: 
primeiro, $G_{k}$ é podado utilizando suas arestas, o que produz o sub-grafo $G_{k+1},\left|V_{G_{k+1}}\right| \leq\left|V_{G_{k}}\right|$; segundo, $G_{k+1}$ é podado com uma verificação de todos os seus vértices. Apesar de que o tempo computacional assintótico deste procedimento é equivalente a uma inspeção direta de todos os vértices de $G_{k}$, na prática esta poda em duas etapas requer um menor tempo computacional, tendo em vista que a poda utilizando as arestas do sub-grafo é computacionalmente mais barata que uma poda que inspeciona todos os seus vértices. Por exemplo, na simulação mostrada nas figuras 4.104.13, 11101 é removido do grafo utilizando as arestas (figuras 4.12(b)-4.12(c)) e 11101 é removido do grafo utilizando a inspeção de vértices (figuras 4.13(a)-4.13(b)). Além disso, na simulação das figuras 4.3-4.9 toda remoção de vértices é feita utilizando apenas as arestas.

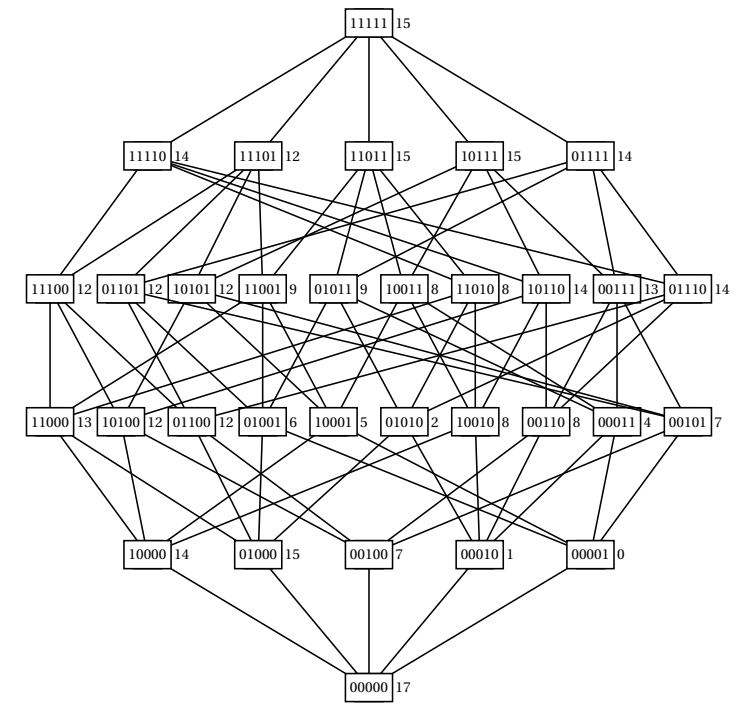

(a)

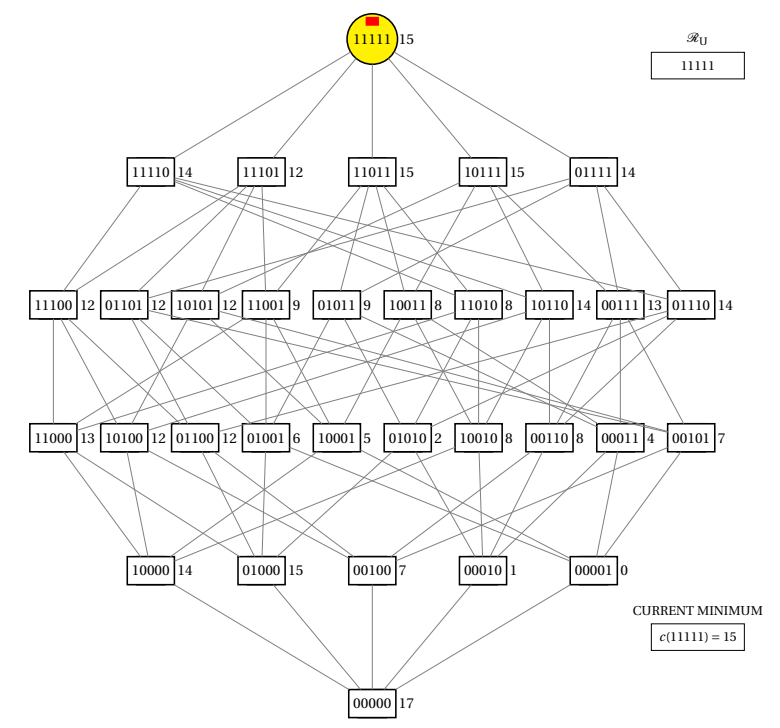

(b)

Figura 4.10: uma outra simulação de UCS, que mostra uma situação em que não bastam as arestas do sub-grafo para realizar uma poda. Neste caso, é necessário fazer a poda em duas etapas: primeiro, a remoção utiliza as arestas; segundo, são inspecionados todos os vértices do sub-grafo obtido da primeira etapa.

- Figura 4.10(a): uma instância com cinco elementos e função-custo $c$, esta última definida pelos números ao lado dos nós;

- Figura 4.10(b): o algoritmo adiciona no grafo 11111, um elemento que é maximal no espaço de busca, isto é, não existe elemento no espaço de busca que contém propriamente 11111. Uma vez que 11111 é maximal, ele também é incluído na coleção de restrições superiores e é assinalado com a marca de restrição superior; 


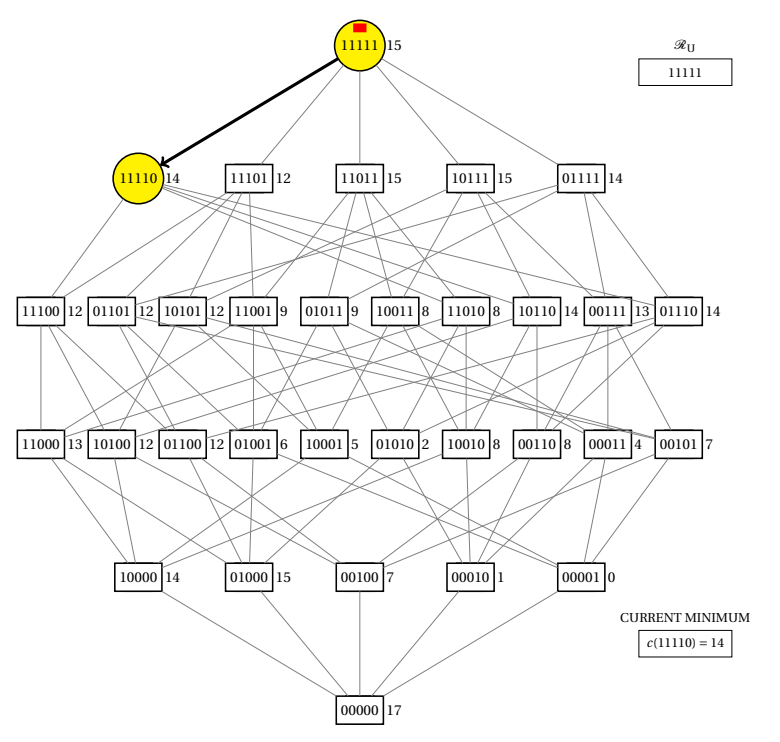

(a)

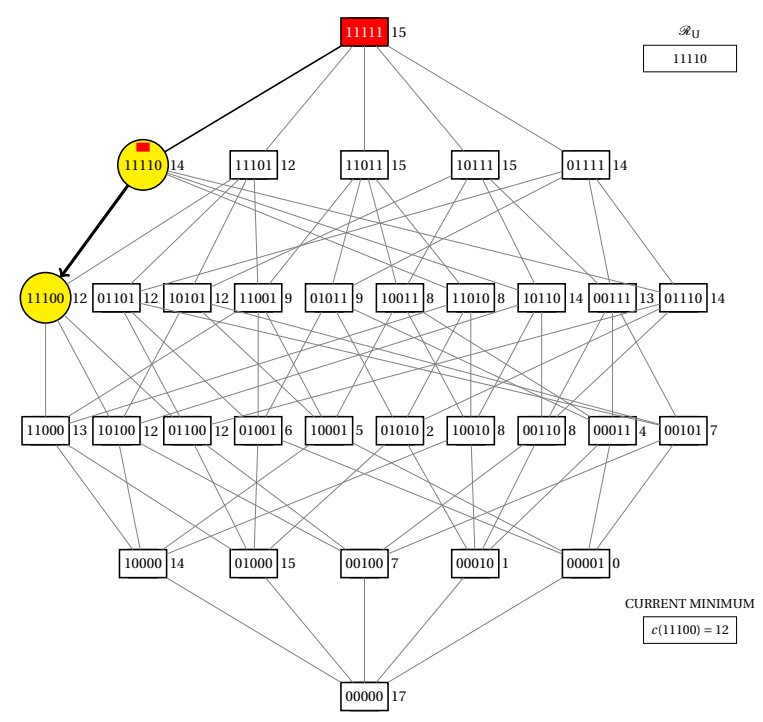

(c)

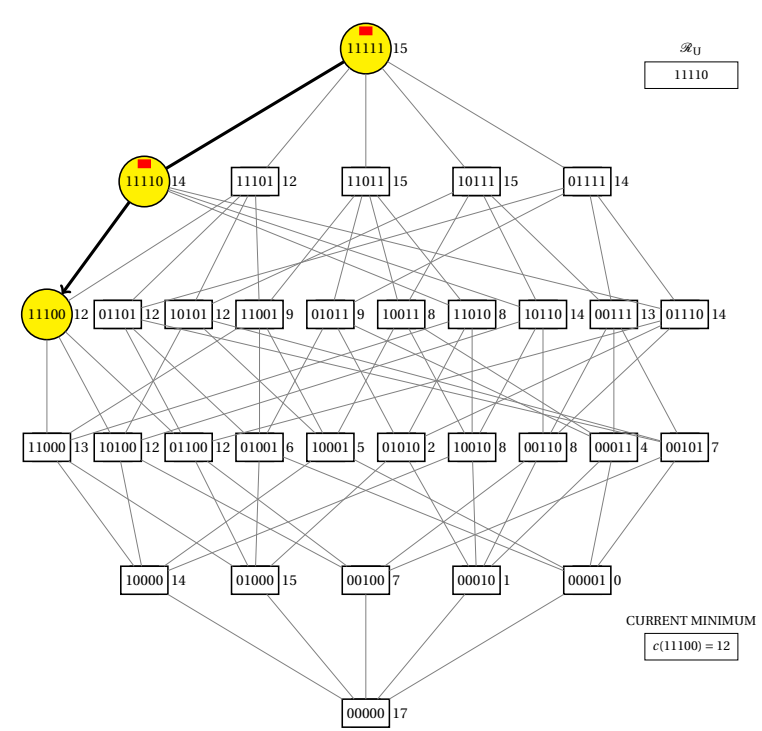

(b)

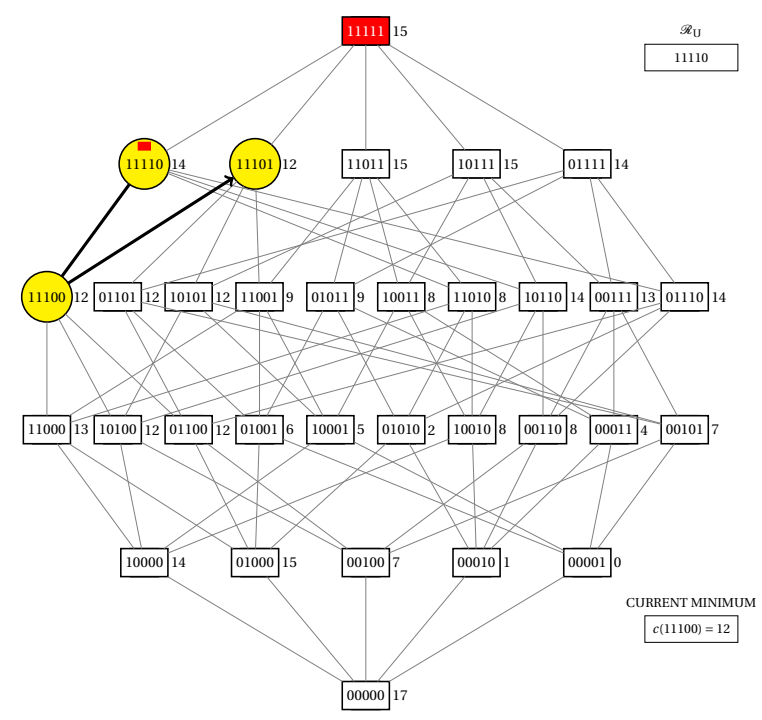

(d)

Figura 4.11: continuação da simulação da figura 4.10.

- Figura 4.11(a): é visitado 11110, um elemento que é adjacente a 11111. $c(11110) \leq c(11111)$, então 11110 é adicionado no grafo;

- Figura 4.11(b): é visitado o elemento 11100, adjacente a 11110. $c(11100)<c(11110)$, portanto 11100 é adicionado no grafo. Além disso, devido à proposição 4.2.2, 11110 é assinalado com a marca de restrição superior e é incluído na coleção de restrições superiores;

- Figura 4.11(c): 11111 inclui propriamente um elemento da coleção de restrições superiores (11110), portanto 11111 é removido do grafo. Esta remoção pode ser realizada utilizando as arestas do grafo;

- Figura 4.11(d): é visitado 11101, um elemento adjacente a 11100; 


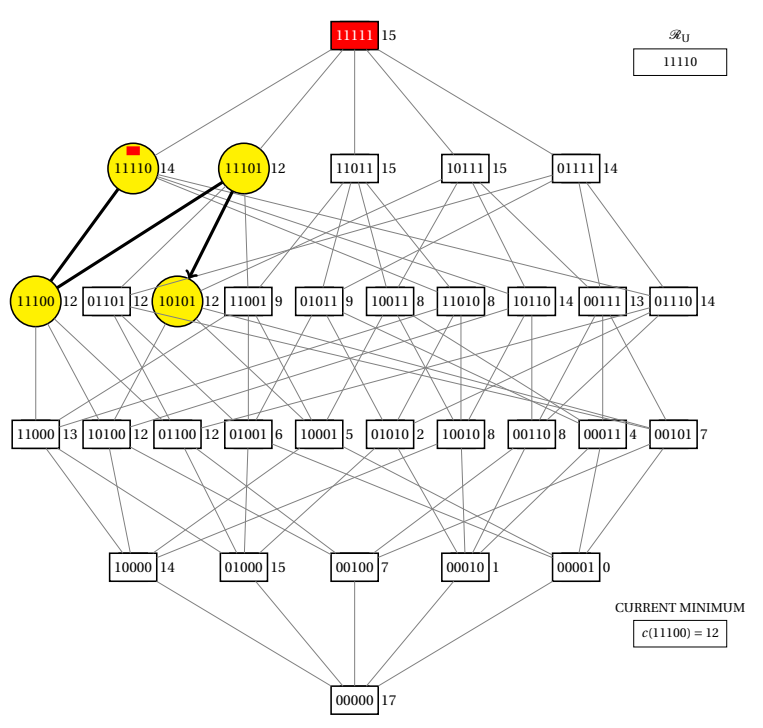

(a)

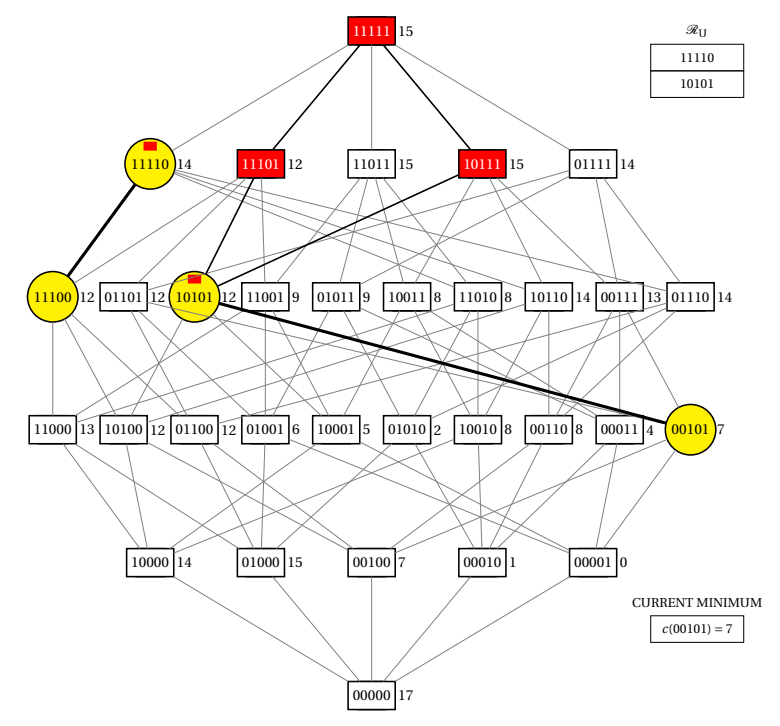

(c)

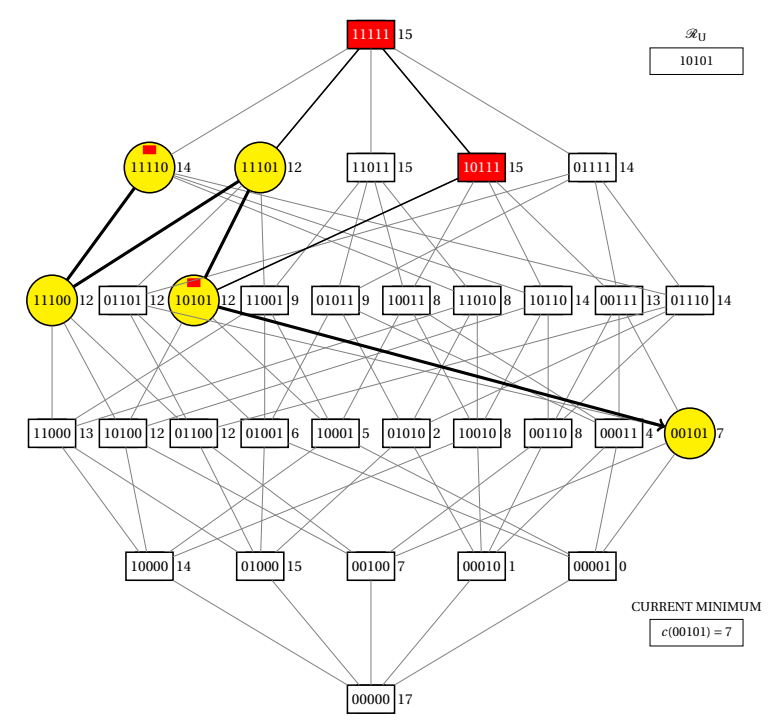

(b)

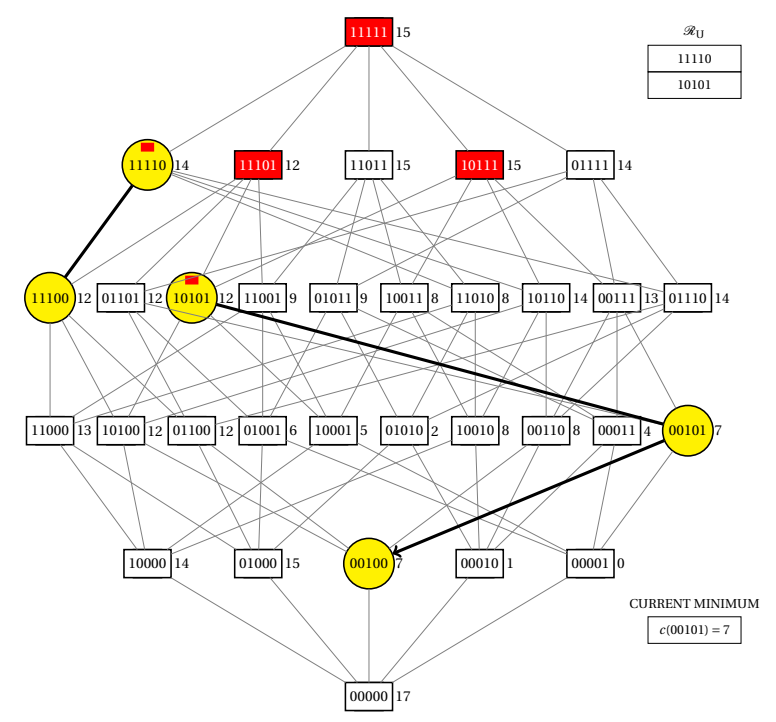

(d)

Figura 4.12: continuação da simulação da figura 4.11.

- Figura 4.12(a): é visitado 10101, um elemento adjacente a 10101;

- Figura 4.12(b): é visitado 00101, adjacente a 10101. $c(00101)<c(10101)$, portanto 10101 é adicionado no grafo. Além disso, devido à proposição 4.2.2, 11110 é assinalado com a marca de restrição superior e é incluído na coleção de restrições superiores;

- Figura 4.12(c): 11101 contém propriamente um elemento da coleção de restrições superiores (10101), portanto 11101 é removido do grafo. Esta remoção pode ser realizada utilizando as arestas do grafo;

- Figura 4.12(d): é visitado 00100, um elemento adjacente a 00101; 


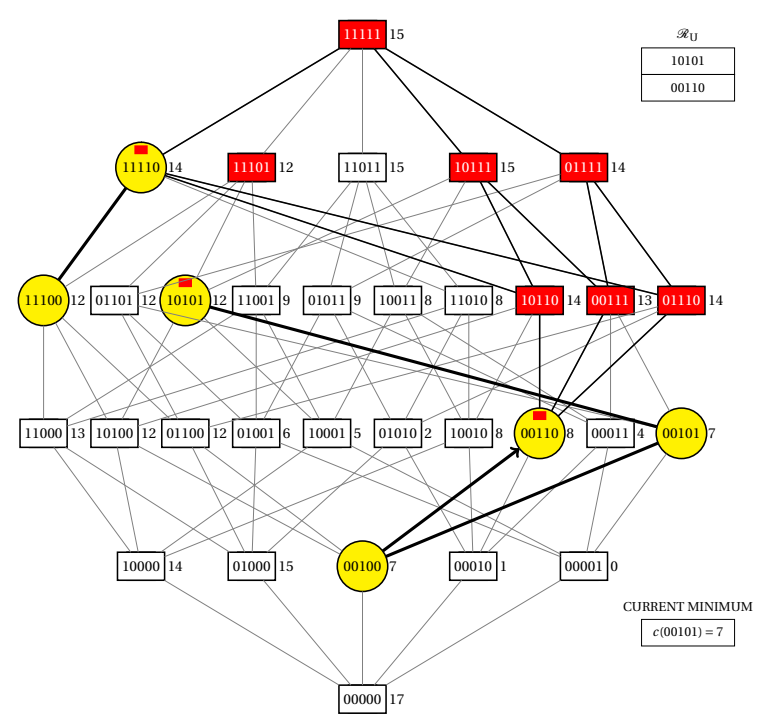

(a)

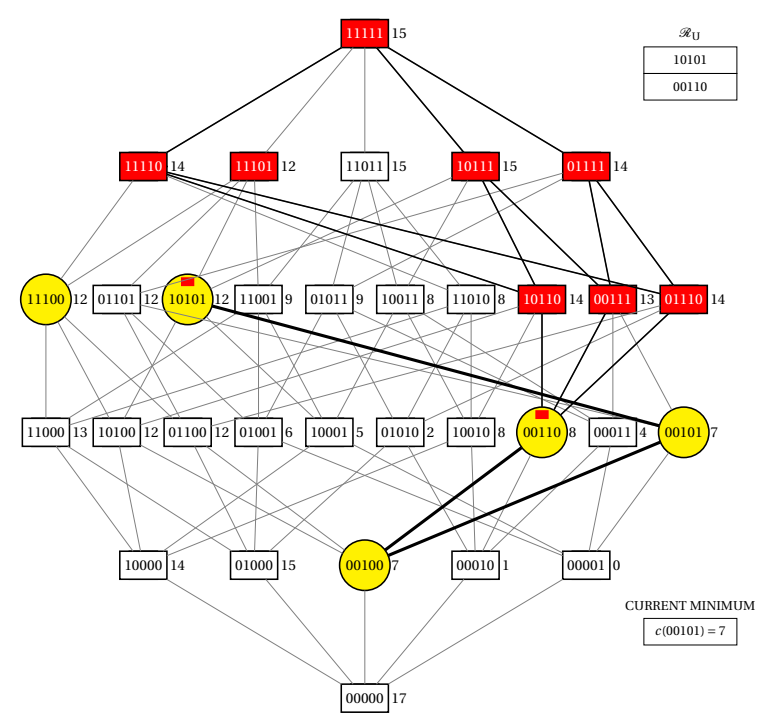

(b)

Figura 4.13: continuação da simulação da figura 4.12.

- Figura 4.13(a): o elemento 00110, adjacente a 00100, é visitado; uma vez que $c(00110)>$ c(00100), 00110 é incluído na coleção de restrições superiores e é assinalado com a marca de restrição superior;

- Figura 4.13(b): 11110 contém propriamente um elemento da coleção de restrições superiores (00110), desta forma 11110 é removido do grafo. Esta remoção não pode ser realizada utilizando as arestas do grafo, haja vista que não existe caminho no grafo entre 11110 e 00110.

Apresentaremos agora cinco sub-rotinas que são utilizadas pelo algoritmo principal de busca em profundidade: a primeira faz seleção de um elemento adjacente não-visitado; a segunda e a terceira fazem, respectivamente, poda inferior e superior do grafo; a quarta providencia poda do grafo levando em consideração as condições das proposições 4.2.1 e 4.2.2; a quinta faz a remoção do grafo.

Descrição de algoritmo. Select-Unvisited-Adjacent recebe um nó Y, uma coleção de nós $\mathcal{G}$, um conjunto finito e não-vazio $S$, uma coleção de restrições inferiores $\mathcal{R}_{L}$ e uma coleção de restrições superiores $\mathcal{R}_{U}$. Esta sub-rotina busca por um elemento adjacente não-visitado a $\mathbf{Y}$ [vertex]. Se um elemento adjacente não-visitado $X$ é encontrado, então esta sub-rotina devolve um nó $\mathbf{X}$ contendo o mesmo; caso contrário, ela devolve NIL. Durante a busca, Y é atualizado seguindo as regras (i) e (iii); dessa forma, esta sub-rotina também devolve $\mathbf{Y}$. 


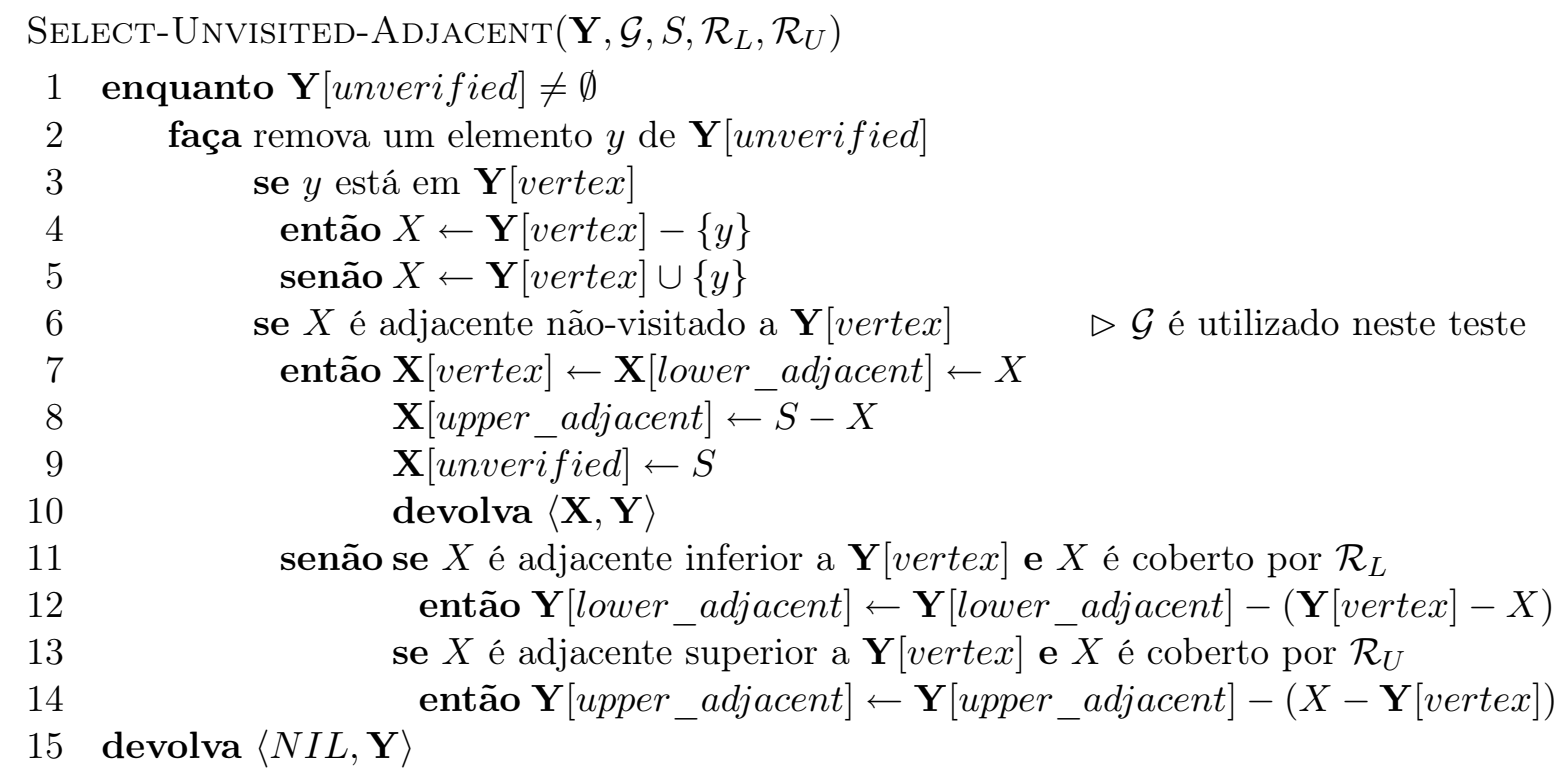

Durante a simulação mostrada a partir da figura 4.3, esta sub-rotina é equivalente às verificações se um elemento do reticulado Booleano vai ser visitado ou não (cada verificação é assinalada com uma flecha e é equivalente a uma iteração desta sub-rotina) apresentadas nas figuras 4.4(a) $($ com Y $[$ vertex $]=00000), 4.4(\mathrm{~b}),(10000), 4.4(\mathrm{~d}),(11000$, duas chamadas desta sub-rotina $), 4.5(\mathrm{~b})$ (11100, duas chamadas), 4.5(d) (11101, duas chamadas), 4.6(a) (10101), 4.6(c) (10001), 4.7(a) (00001, três chamadas), 4.7(d) (11110), 4.8(a) (11010, duas chamadas), 4.8(c) (10010, duas chamadas), 4.9(a) (00010, três chamadas) e 4.9(d) (11100, uma chamada que devolve NIL).

Análise de complexidade de tempo. O laço enquanto das linhas 1-14 itera no máximo $n+1$ vezes. A cada iteração, a verificação de nó na linha 6 consome $O(n|\mathcal{G}|)$ unidades de tempo, a verificação do espaço de busca na mesma linha consome $O\left(n\left(\left|\mathcal{R}_{L}\right|+\left|\mathcal{R}_{U}\right|\right)\right)$ unidades de tempo, e as verificações na linha 11 e na linha 13 consomem, respectivamente, $O\left(n\left|\mathcal{R}_{L}\right|\right)$ e $O\left(n\left|\mathcal{R}_{U}\right|\right)$ unidades de tempo. Finalmente, se as linhas 7-10 são executadas, um novo nó $\mathbf{X}$ é criado; logo, pela regra (v), o valor $c(X)$ é computado e armazenado em $\mathbf{X}[$ cost $]$, o que demanda $O(f(n))$ unidades de tempo. Portanto, Select-Unvisited-Adjacent consome $O\left(f(n)+n^{2}\left(\left|\mathcal{R}_{L}\right|+\left|\mathcal{R}_{U}\right|+|\mathcal{G}|\right)\right)$ unidades de tempo.

Descrição de algoritmo. Lower-PrUNing recebe um nó $\mathbf{Y}$, uma coleção de nós $\mathcal{G}$ e uma coleção de restrições inferiores $\mathcal{R}_{L}$. Esta sub-rotina inclui $\mathbf{Y}[$ vertex $]$ em $\mathcal{R}_{L}$ utilizando a sub-rotina Update-Lower-Restriction. Ela também remove de $\mathcal{G}$ todo nó $\mathbf{X}$ tal que $\mathbf{X}$ [vertex] seja contido propriamente em $\mathbf{Y}\left[\right.$ vertex]. Finalmente, esta sub-rotina devolve as coleções atualizadas $\mathcal{G}$ e $\mathcal{R}_{L}$. 


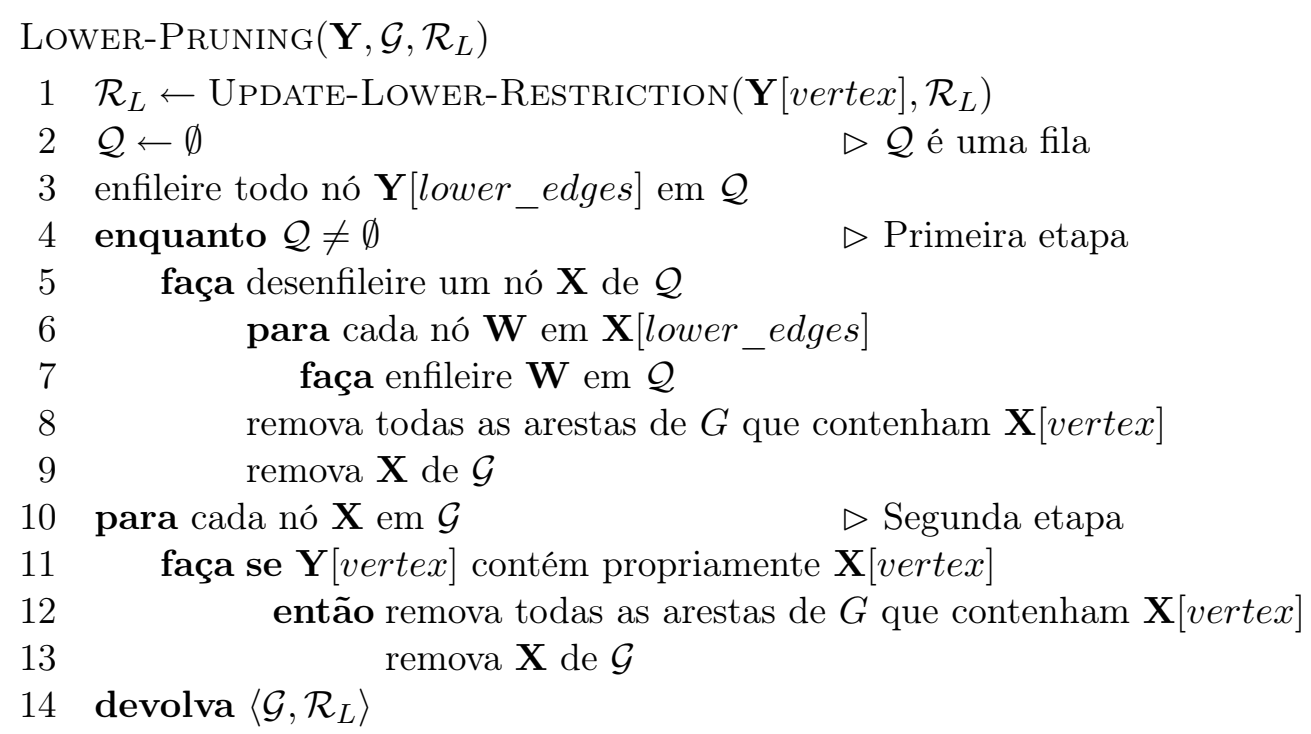

No pseudo-código anterior, a remoção de uma aresta $\{\mathbf{X}[$ vertex $], Z\}$ tal que $Z$ é um vértice de $G$ consiste na aplicação da regra (iv).

Durante a simulação mostrada a partir da figura 4.3, esta sub-rotina é equivalente aos assinalamentos de marca de restrição inferior, às atualizações da coleção de restrições inferiores, e às remoções de nós apresentados nas figuras 4.4(b)-4.4(c) (com Y $[$ vertex] = 10000), 4.4(d) (01000), $4.4(\mathrm{~d})-4.5(\mathrm{a})(11000), 4.5(\mathrm{~b})-4.5(\mathrm{c})(11100), 4.7(\mathrm{~b})-4.7(\mathrm{c})(00001)$ e $4.9(\mathrm{~b})-4.9(\mathrm{c})$ (00010).

Análise de complexidade de tempo. Na primeira etapa, as operações de enfileiramento e de desenfileiramento consomem, cada uma, $O(1)$ unidades de tempo. Uma remoção de um nó $\mathbf{X}$ de $\mathcal{G}$ consome $O(1)$ unidades de tempo, e uma remoção de todos os nós de $\mathbf{X}$ [lower_edges] e $\mathbf{X}[$ upper_edges] precisa de $O(n)$ unidades de tempo. Entretanto, uma vez que o grafo $G$ é uma floresta (i.e., um grafo sem circuitos), $|G|$ tem no máximo $|V|-1$ arestas, o que implica que $|V|-1=O(|\mathcal{G}|)$ é um limite superior para todas as possíveis remoções de arestas. Uma vez que $|V|$ também é o limite superior para o número de iterações do laço das linhas 4-9, a primeira etapa consome $O(|\mathcal{G}|)$ unidades de tempo.

Na segunda etapa, o laço das linhas $10-13$ itera $O(|\mathcal{G}|)$ vezes. A verificação de cada nó em $\mathcal{G}$ consome $O(n)$ unidades de tempo, e a remoção de todos os nós em $\mathbf{X}$ [lower_edges] e $\mathbf{X}$ [upper_edges] requer $O(n)$ unidades de tempo. Portanto, a segunda etapa consome $O(n|\mathcal{G}|)$ unidades de tempo.

Uma vez que a única chamada de UpDATE-LOWER-RESTRICTION requer $O\left(n\left|\mathcal{R}_{L}\right|\right)$ unidades de tempo, no total Lower-Pruning consome $O\left(n\left(\left|\mathcal{R}_{L}\right|+|\mathcal{G}|\right)\right)$ unidades de tempo.

Algoritmo dual. UpPer-Pruning recebe um nó $\mathbf{Y}$, uma coleção de nós $\mathcal{G}$ e uma coleção de restrições superiores $\mathcal{R}_{U}$. Esta sub-rotina inclui o elemento $\mathbf{Y}$ [vertex $]$ em $\mathcal{R}_{U}$ utilizando a sub-rotina Update-Upper-Restriction. Ela também remove de $\mathcal{G}$ todo nó $\mathbf{X}$ tal que $\mathbf{X}$ [vertex $]$ contenha propriamente $\mathbf{Y}[$ vertex $]$. Finalmente, esta sub-rotina devolve as coleções atualizadas $\mathcal{G}$ e $\mathcal{R}_{U}$.

Descrição de algoritmo. Graph-Pruning recebe um par de nós $\mathbf{X}$ e $\mathbf{Y}$, uma coleção de nós $\mathcal{G}$, uma coleção de restrições inferiores $\mathcal{R}_{L}$, uma coleção de restrições superiores $\mathcal{R}_{U}$ e uma função custo $c$ decomponível em curvas em U. Esta sub-rotina verifica as condições das proposições $4.2 .1 \mathrm{e}$ 4.2.2, podando o grafo e atualizando as coleções de restrições de acordo com as mesmas. Finalmente, esta sub-rotina devolve as coleções atualizadas $\mathcal{G}, \mathcal{R}_{L}$ e $\mathcal{R}_{U}$. 
$\operatorname{Graph-PRUning}\left(\mathbf{X}, \mathbf{Y}, \mathcal{G}, \mathcal{R}_{L}, \mathcal{R}_{U}, c\right)$

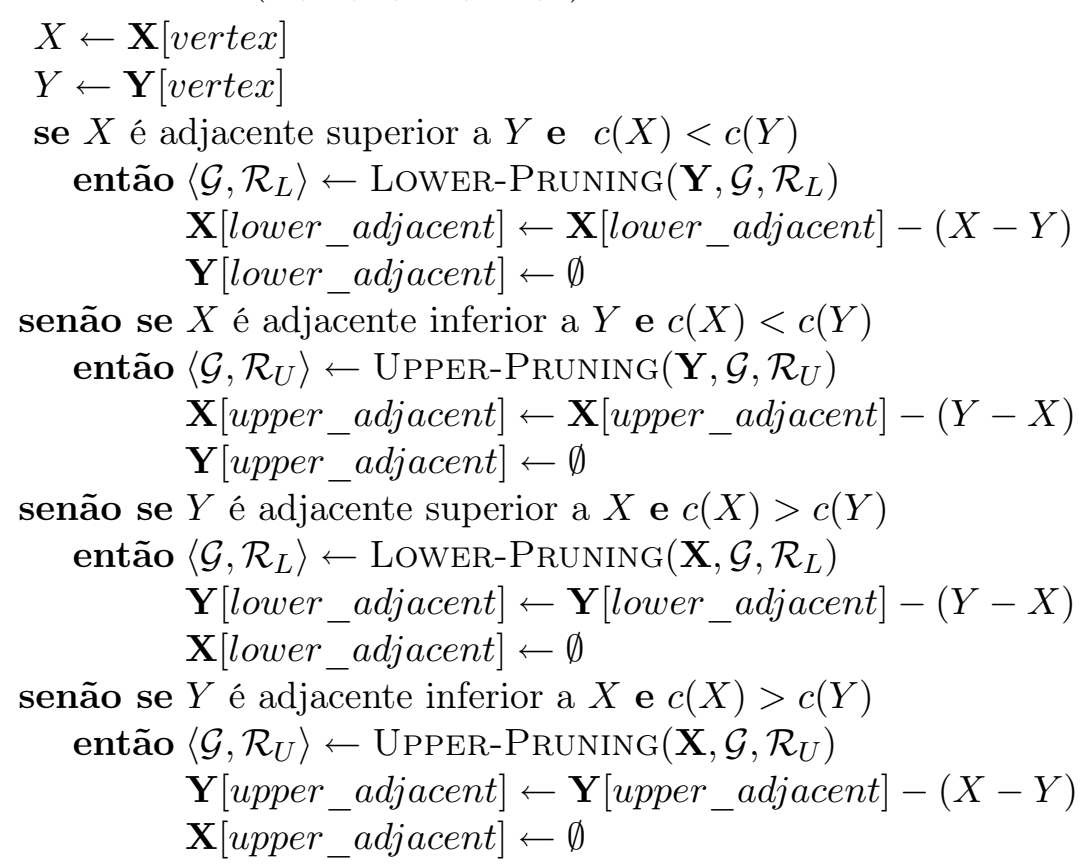

Durante a simulação mostrada a partir da figura 4.3, esta sub-rotina é equivalente a todos os assinalamentos de marca de restrições, atualizações das coleções de restrições inferiores e remoções de nós, exceto por: 4.7(b)-4.7(c) (com Y $[$ vertex $]=00001)$ e 4.9(b)-4.9(c) (00010), que são podas devido às proposições 4.2 .3 e $4.2 .4 ; 4.9$ (d) (11100), que é uma poda feita pela quinta sub-rotina, quando não há vértice no grafo que tenha um elemento adjacente não-visitado.

Análise de complexidade de tempo. A poda nas linhas 4 e 12 leva $O\left(n\left(\left|\mathcal{R}_{L}\right|+|\mathcal{G}|\right)\right)$ (i.e., a complexidade de tempo de Lower-Pruning), e nas linhas 8 e 16 leva $O\left(n\left(\left|\mathcal{R}_{U}\right|+|\mathcal{G}|\right)\right)$ (i.e., a complexidade de tempo de UpPer-Pruning). Pela regra (v), cada chamada da função custo leva $O(1)$. Portanto, Graph-Pruning requer $O\left(n\left(\left|\mathcal{R}_{L}\right|+\left|\mathcal{R}_{U}\right|+|\mathcal{G}|\right)\right)$ unidades de tempo.

Descrição de algoritmo. REMOVE-GRAPH recebe uma coleção de nós $\mathcal{G}$, uma coleção de restrições inferiores $\mathcal{R}_{L}$ e uma coleção de restrições superiores $\mathcal{R}_{U}$. Para cada nó $\mathbf{X}$ de $\mathcal{G}$, se $\mathbf{X}[$ lower_adjacent $]=\emptyset(\mathbf{X}[$ upper_adjacent $]=\emptyset)$, então esta sub-rotina inclui $\mathbf{X}[$ vertex $]$ em $\mathcal{R}_{U}\left(\mathcal{R}_{L}\right)$ utilizando Update-Upper-Restriction (Update-Lower-Restriction). Finalmente, esta sub-rotina devolve as coleções atualizadas $\mathcal{R}_{L}$ e $\mathcal{R}_{U}$.

Durante a simulação mostrada a partir da figura 4.3, esta sub-rotina é equivalente a atualização da coleção de restrições superiores e a remoção de nó apresentada na figura 4.9(d) (com X $\mathbf{X}$ vertex] = 11100).

Análise de complexidade de tempo. Uma vez que Update-Lower-Restriction (no caso dual, UpdATE-UpPER-RESTRICTION) requer $O\left(n\left|\mathcal{R}_{L}\right|\right)\left(O\left(n\left|\mathcal{R}_{U}\right|\right)\right)$ unidades de tempo, que até $|\mathcal{G}|$ nós podem ter seus respectivos elementos incluídos nas coleções de restrições, e que varrer a coleção $\mathcal{G}$ é linear em seu tamanho, Remove-Graph consome $O\left(n|\mathcal{G}|\left(\left|\mathcal{R}_{L}\right|+\left|\mathcal{R}_{U}\right|\right)\right)$ unidades de tempo.

\section{O algoritmo principal da busca em profundidade}

Sejam $X$ um elemento de $\mathcal{P}(S)$ e $c$ uma função custo decomponível em curvas em U. Queremos realizar uma busca em profundidade em $\mathcal{X}\left(\mathcal{R}_{L}, \mathcal{R}_{U}\right)$ que se inicie em $X$ e que tenha as seguintes propriedades: 
- dado um elemento $Y$ de $\mathcal{P}(S)$, o critério de exploração (depth-first criterion) é visitar um elemento $X$ adjacente a $Y$ tal que $X \in \mathcal{X}\left(\mathcal{R}_{L}, \mathcal{R}_{U}\right)$ e $c(X) \leq c(Y)$;

- o critério de parada é a regra (ii), isto é, quando um vértice $Y$ do grafo não possui elemento adjacente não-visitado;

- $\mathcal{X}\left(\mathcal{R}_{L}, \mathcal{R}_{U}\right)$ é podado através da atualização das coleções de restrições. Esta atualização leva em consideração as proposições 4.2.1, 4.2.2, 4.2.3 e 4.2.4 para remover elementos do espaço de busca.

Descrição de algoritmo. DFS recebe um conjunto finito e não-vazio $S$, um nó $\mathbf{M}$, uma coleção de restrições inferiores $\mathcal{R}_{L}$, uma coleção de restrições superiores $\mathcal{R}_{U}$ e uma função custo $c$ decomponível em curvas em U. Esta sub-rotina realiza uma busca em profundidade utilizando as estruturas de dados, sub-rotinas, regras e propriedades descritas. Esta sub-rotina inspeciona um pedaço do espaço de busca corrente $\mathcal{X}\left(\mathcal{R}_{L}, \mathcal{R}_{U}\right)$, atualizando os mínimos correntes; ela também atualiza o espaço de busca corrente para $\mathcal{X}\left(\mathcal{R}^{\prime}{ }_{L}, \mathcal{R}^{\prime}{ }_{U}\right) \subseteq \mathcal{X}\left(\mathcal{R}_{L}, \mathcal{R}_{U}\right)$. Portanto, esta sub-rotina devolve $\mathcal{R}_{L}^{\prime}$, $\mathcal{R}_{U}^{\prime}$ e uma coleção $\mathcal{M}$ contendo todo elemento $X$ de $\mathcal{X}\left(\mathcal{R}_{L}, \mathcal{R}_{U}\right)-\mathcal{X}\left(\mathcal{R}^{\prime}{ }_{L}, \mathcal{R}_{U}^{\prime}\right)$ tal que $X$ é mínimo $\operatorname{em} \mathcal{X}\left(\mathcal{R}_{L}, \mathcal{R}_{U}\right)-\mathcal{X}\left(\mathcal{R}_{L}^{\prime}, \mathcal{R}_{U}^{\prime}\right)$.

$\operatorname{DFS}\left(\mathbf{M}, S, \mathcal{R}_{L}, \mathcal{R}_{U}, c\right)$

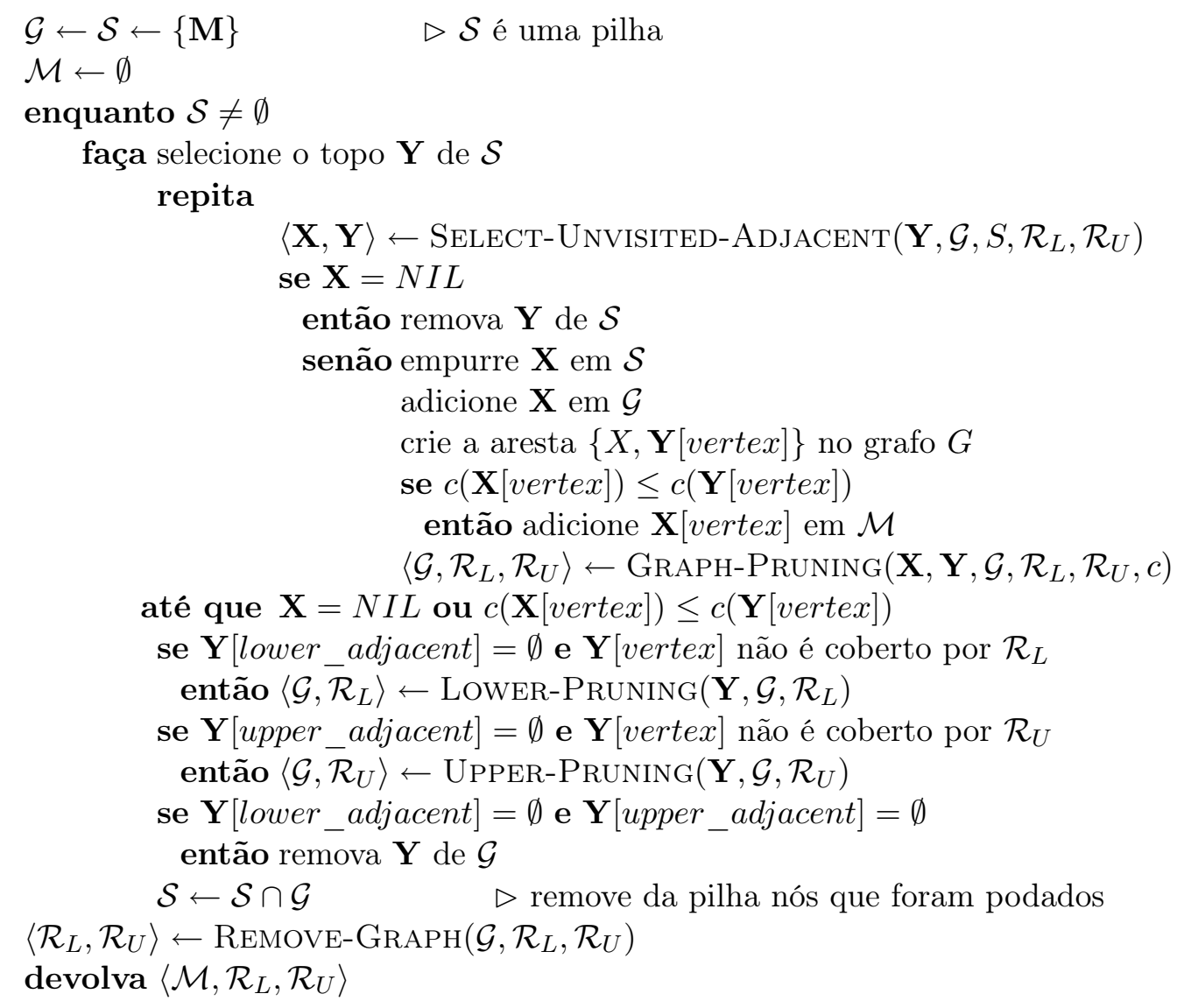

No pseudo-código anterior, a criação de uma aresta $\{X, \mathbf{Y}[$ vertex $]\}$ consiste na aplicação da regra (iv).

Durante a simulação mostrada a partir da figura 4.3, os passos das figuras 4.4(a)-4.9(d) são equivalentes a uma execução de DFS com parâmetros $\mathbf{M}[$ vertex $]=\mathbf{M}[$ lower_adjacent $]=00000$, $\mathbf{M}[$ upper_adjacent $]=\mathbf{M}[$ unverified $]=S=11111, \mathbf{M}[$ upper_edges $]=\mathbf{M}[$ lower_edges $]=$ $\mathcal{R}_{L}=\mathcal{R}_{U}=\emptyset$ e $c$ definida pelos números ao lado dos nós. 
Análise de complexidade de tempo. Consideremos que $\left|\mathcal{R}_{L}\right|,\left|\mathcal{R}_{U}\right|$ e $|\mathcal{G}|$ representam os maiores tamanhos que essas coleções atingem durante as iterações da sub-rotina. Definiremos $g$ como o número de vezes que um nó é inserido em $\mathcal{G}$, o qual é equivalente ao número de vezes que um nó é empurrado em $\mathcal{S}$. Valem as seguintes afirmações:

- uma vez que a pilha $\mathcal{S}$ também é uma coleção de nós, a mesma também é implementada como uma lista duplamente encadeada, portanto cada seleção de nó que está no topo de $\mathcal{S}$ na linha 4 pode ser feita em $O(1)$;

- a chamada de Select-Unvisited-Adjacent na linha 6 demanda $O\left(f(n)+n^{2}\left(\left|\mathcal{R}_{L}\right|+\left|\mathcal{R}_{U}\right|+\right.\right.$ $|\mathcal{G}|)$ ) unidades de tempo;

- pela regra (v), cada chamada da função custo na linha 12 demanda $O(1)$;

- a poda na linha 14 consome $O\left(f(n)+n\left(\left|\mathcal{R}_{L}\right|+\left|\mathcal{R}_{U}\right|+|\mathcal{G}|\right)\right)$ unidades de tempo;

- uma vez que o laço repita das linhas 5-15 itera no máximo $n+1$ vezes, a complexidade de tempo deste laço é $O\left(n\left(f(n)+\left(n^{2}\left(\left|\mathcal{R}_{L}\right|+\left|\mathcal{R}_{U}\right|+|\mathcal{G}|\right)\right)+\left(n\left(\left|\mathcal{R}_{L}\right|+\left|\mathcal{R}_{U}\right|+|\mathcal{G}|\right)\right)\right)\right)=$ $O\left(f(n) n+n^{3}\left(\left|\mathcal{R}_{L}\right|+\left|\mathcal{R}_{U}\right|+|\mathcal{G}|\right)\right)$

- utilizando o método agregado da Análise Amortizada (Cormen et al., capítulo 17 [CLRS01]), verificamos que o laço das linhas $5-15$ de fato consome $O\left(f(n) n+n^{2}\left(\left|\mathcal{R}_{L}\right|+\left|\mathcal{R}_{U}\right|+|\mathcal{G}|\right)\right)$ unidades de tempo, haja vista que o tamanho de $\mathbf{Y}[$ unvisited], o qual é um limite superior para o número de iterações de SElect-Unvisited-Adjacent, decresce a cada chamada dessa sub-rotina;

- utilizando uma vez mais o método agregado, verificamos que o laço enquanto das linhas 3-22 e o laço repita das linhas $5-15$ juntos iteram no máximo $g(n+1)$ vezes. Portanto, todas as iterações do laço repita levam $O\left(f(n) g n+g n^{2}\left(\left|\mathcal{R}_{L}\right|+\left|\mathcal{R}_{U}\right|+|\mathcal{G}|\right)\right)$;

- uma vez que a função custo $c$ é computada apenas uma vez para cada vértice do grafo (regra $(\mathrm{v})), c$ é computada $g-1$ vezes (i.e., $c$ é computada para cada nó que é adicionado em $\mathcal{G}$, com exceção do nó $\mathbf{M}$ recebido pela sub-rotina). Portanto, todas as iterações do laço repita levam $O\left(f(n) g+g n^{2}\left(\left|\mathcal{R}_{L}\right|+\left|\mathcal{R}_{U}\right|+|\mathcal{G}|\right)\right)$;

- a poda nas linhas 17 e 19 ocorre no máximo uma vez para cada nó adicionado em $\mathcal{G}$. Portanto, pelo método agregado, todas as podas deste tipo juntas consomem $O\left(\operatorname{gn}\left(n\left(\left|\mathcal{R}_{L}\right|+\left|\mathcal{R}_{U}\right|\right)+|\mathcal{G}|\right)\right)$;

- a chamada de Remove-Graph na linha 23 leva $O\left(n|\mathcal{G}|\left(\left|\mathcal{R}_{L}\right|+\left|\mathcal{R}_{U}\right|\right)\right)$.

Pelas últimas três afirmações, a complexidade de tempo de DFS como uma função de $f(n), g, n$, $\left|\mathcal{R}_{L}\right|$ e $\left|\mathcal{R}_{U}\right|$ é dada por

$$
\begin{aligned}
T_{\mathrm{DFS}}= & O\left(f(n) g+g n^{2}\left(\left|\mathcal{R}_{L}\right|+\left|\mathcal{R}_{U}\right|+|\mathcal{G}|\right)\right)+O\left(g n\left(n\left(\left|\mathcal{R}_{L}\right|+\left|\mathcal{R}_{U}\right|\right)+|\mathcal{G}|\right)\right)+ \\
& +O\left(n|\mathcal{G}|\left(\left|\mathcal{R}_{L}\right|+\left|\mathcal{R}_{U}\right|\right)\right) \\
= & O\left(f(n) g+g n^{2}\left(\left|\mathcal{R}_{L}\right|+\left|\mathcal{R}_{U}\right|+|\mathcal{G}|\right)+O\left(n|\mathcal{G}|\left(\left|\mathcal{R}_{L}\right|+\left|\mathcal{R}_{U}\right|\right)\right)\right. \\
= & O\left(f(n) g+g n^{2}\left(\left|\mathcal{R}_{L}\right|+\left|\mathcal{R}_{U}\right|+g\right)\right)+O\left(n g\left(\left|\mathcal{R}_{L}\right|+\left|\mathcal{R}_{U}\right|\right)\right) \\
= & O\left(f(n) g+g n^{2}\left(\left|\mathcal{R}_{L}\right|+\left|\mathcal{R}_{U}\right|+g\right)\right)
\end{aligned}
$$

\subsubsection{O algoritmo principal}

Apresentaremos agora o algoritmo principal. 
atingem durante as iterações do algoritmo. O algoritmo UCS consome

$$
\begin{aligned}
& T_{\mathrm{UCS}}=O\left((k-j) n^{2}\left(\left|\mathcal{R}_{L}\right|+\left|\mathcal{R}_{U}\right|\right)\right)+O(f(n) j)+\sum_{i=1}^{j} O\left(f(n) g_{i}+g_{i} n^{2}\left(\left|\mathcal{R}_{L}\right|+\left|\mathcal{R}_{U}\right|+g_{i}\right)\right) \\
& =O\left((k-j) n^{2}\left(\left|\mathcal{R}_{L}\right|+\left|\mathcal{R}_{U}\right|\right)\right)+O(f(n) j)+ \\
& +O\left(f(n) m+m n^{2}\left(\left|\mathcal{R}_{L}\right|+\left|\mathcal{R}_{U}\right|+m\right)\right) \\
& \text { (uma vez que } \sum_{i=1}^{j} g_{i}=m \text { ) } \\
& =O\left(f(n) m+f(n) j+(k-j) n^{2}\left(\left|\mathcal{R}_{L}\right|+\left|\mathcal{R}_{U}\right|\right)+m n^{2}\left(\left|\mathcal{R}_{L}\right|+\left|\mathcal{R}_{U}\right|+m\right)\right) \\
& =O\left(f(n) m+f(n) j+n^{2}\left((k-j)\left(\left|\mathcal{R}_{L}\right|+\left|\mathcal{R}_{U}\right|\right)+m\left(\left|\mathcal{R}_{L}\right|+\left|\mathcal{R}_{U}\right|+m\right)\right)\right) \\
& =O\left(f(n) m+n^{2}\left((k-j)\left(\left|\mathcal{R}_{L}\right|+\left|\mathcal{R}_{U}\right|\right)+m\left(\left|\mathcal{R}_{L}\right|+\left|\mathcal{R}_{U}\right|+m\right)\right)\right) \quad \text { (uma vez que } j \leq m \text { ) } \\
& =O\left(f(n) m+n^{2}\left((k-j+m)\left(\left|\mathcal{R}_{L}\right|+\left|\mathcal{R}_{U}\right|+m\right)\right)\right) \\
& =O\left(f(n) m+n^{2}\left(u\left(\left|\mathcal{R}_{L}\right|+\left|\mathcal{R}_{U}\right|+m\right)\right)\right) \\
& =O\left(f(n) m+n^{2}(u(u+u+m))\right) \\
& =O\left(f(n) m+n^{2}(u(u+u))\right) \\
& =O\left(f(n) m+n^{2} u^{2}\right) \\
& \text { (uma vez que } k-j+m=u \text { ) } \\
& \text { (uma vez que }\left|\mathcal{R}_{L}\right| \leq u \text { e }\left|\mathcal{R}_{U}\right| \leq u \text { ) } \\
& \text { (uma vez que } m \leq u \text { ) }
\end{aligned}
$$

unidades de tempo. Como a execução deste algoritmo pode explorar uma fração do espaço de busca que é proporcional ao tamanho de um reticulado Booleano de grau $n$, o limite superior real para o tempo computacional assintótico de UCS é $O\left(f(n) 2^{n}+n^{2} 2^{n}\right)=O\left(\left(f(n)+n^{2}\right) 2^{n}\right)$.

\subsection{Experimentos}

Nesta seção apresentaremos uma avaliação experimental do algoritmo UCS. Esta avaliação foi feita através de experimentos ótimos e sub-ótimos segundo a descrição feita na seção 2.2.2; tais experimentos utilizaram como função custo a equação 3.1, e como algoritmos UCS, SFFS e ES (i.e., a busca exaustiva descrita no apêndice B).

Na tabela 4.1 mostramos os resultados do experimento ótimo. Do ponto de vista semântico, SFFS teve um desempenho pobre neste experimento: primeiro, para o grupo das menores instâncias (7) ele encontrou uma melhor solução em apenas 48 de 100 instâncias; segundo, com o incremento do tamanho das instâncias, era menos provável que SFFS fornecesse uma solução ótima. ES e UCS são equivalentes, uma vez que ambos fornecem uma solução ótima. Do ponto de vista de desempenho computacional, para grupos de instâncias de tamanho até 11, UCS computou menos vezes a função custo do que SFFS. Por outro lado, UCS gasta maior tempo computacional que SFFS e mesmo que ES, sendo que este último sempre visita todos os elementos do espaço de busca.

Na tabela 4.2 apresentamos os resultados da primeira e da segunda etapa do experimento subótimo, enquanto que na tabela 4.3 resumimos os resultados da terceira etapa. UCS tem uma melhor semântica do que SFFS e ES, e ES por sua vez tem uma semântica melhor do que SFFS.

\subsubsection{Análise dos experimentos}

Apresentaremos as análises dos experimentos na seguinte ordem: primeiro, analisaremos o uso de memória do algoritmo UCS, o qual é medido a partir dos resultados obtidos nos experimentos ótimos e sub-ótimos apresentados na seção anterior; segundo, analisaremos alguns aspectos da dinâmica do algoritmo UCS, o que também será feito a partir dos resultados dos experimentos da seção anterior.

Para medirmos o uso de memória para cada experimento, executamos o algoritmo UCS em grupos de cem instâncias de mesmo tamanho (uma execução por instância). Para cada instância, armazenamos o tamanho máximo atingido pelo grafo durante a execução do algoritmo. Finalmente, 


\begin{tabular}{|c|c|c|c|c|c|c|c|c|c|c|}
\hline \multicolumn{2}{|c|}{ Instância } & \multicolumn{3}{|c|}{ Tempo (seg) } & \multicolumn{3}{|c|}{ \# Nós computados } & \multicolumn{3}{|c|}{ \# Melhor solução } \\
\hline$|S|$ & $2^{|S|}$ & $\mathrm{ES}$ & $\mathrm{UCS}$ & SFFS & ES & $\mathrm{UCS}$ & SFFS & $\mathrm{ES}$ & $\mathrm{UCS}$ & SFFS \\
\hline 7 & 128 & 0,15 & 0,21 & 0,15 & 128 & 58,12 & 130,15 & 100 & 100 & 48 \\
\hline 8 & 256 & 0,19 & 0,26 & 0,13 & 256 & 99,28 & 181,72 & 100 & 100 & 30 \\
\hline 9 & 512 & 0,25 & 0,34 & 0,16 & 512 & 173,95 & 268,93 & 100 & 100 & 26 \\
\hline 10 & 1024 & 0,31 & 0,44 & 0,15 & 1024 & 243,66 & 390,98 & 100 & 100 & 30 \\
\hline 11 & 2048 & 0,44 & 0,74 & 0,16 & 2048 & 440,05 & 498,96 & 100 & 100 & 17 \\
\hline 12 & 4096 & 0,71 & 1,23 & 0,19 & 4096 & 757,30 & 712,38 & 100 & 100 & 18 \\
\hline 13 & 8192 & 1,23 & 2,67 & 0,23 & 8192 & 1289,91 & 780,11 & 100 & 100 & 12 \\
\hline 14 & 16384 & 2,37 & 6,10 & 0,21 & 16384 & 2216,35 & 882,66 & 100 & 100 & 12 \\
\hline 15 & 32768 & 4,62 & 19,84 & 0,20 & 32768 & 4289,53 & 958,66 & 100 & 100 & 7 \\
\hline 16 & 65536 & 8,61 & 56,96 & 0,25 & 65536 & 8192,95 & 1226,64 & 100 & 100 & 6 \\
\hline 17 & 131072 & 17,28 & 139,15 & 0,23 & 131072 & 13077,23 & 1347,90 & 100 & 100 & 6 \\
\hline 18 & 262144 & 36,66 & 569,75 & 0,45 & 262144 & 25136,01 & 1761,12 & 100 & 100 & 12 \\
\hline
\end{tabular}

Tabela 4.1: comparação entre UCS, SFFS e ExHAUSTIVE-SEARCH (ES), utilizando como função custo a equação 3.1 (i.e., uma redução polinomial de instâncias do problema da soma de subconjuntos, um problema NP-dificil). Apesar de que o tempo computacional de UCS aumenta mais rapidamente que o da busca exaustiva, o primeiro precisa computar menos vezes a função custo do que o segundo.

\begin{tabular}{|c|c|c|c|c|c|}
\hline \multicolumn{2}{|c|}{ Instância } & \multicolumn{3}{|c|}{ \# Nós computados } & \multirow[t]{2}{*}{ Limiar } \\
\hline$\overline{|S|}$ & $2^{|S|}$ & ES & UCS & SFFS & \\
\hline 7 & 128 & 33,88 & 26,16 & 56,43 & 57 \\
\hline 8 & 256 & 64,46 & 31,32 & $\overline{92,75}$ & 93 \\
\hline 9 & 512 & 156,47 & 56,24 & $\overline{163,95}$ & 164 \\
\hline 10 & 1024 & 248,00 & 77,57 & $\overline{239,12}$ & 248 \\
\hline 11 & 2048 & $\overline{386,55}$ & 78,39 & 267,99 & 387 \\
\hline 12 & 4096 & $1 \overline{021,57}$ & 112,84 & 410,32 & 1022 \\
\hline 13 & 8192 & $\overline{1903,63}$ & 134,65 & 643,40 & 1904 \\
\hline 14 & 16384 & $\overline{3491,07}$ & 137,31 & 879,61 & 3492 \\
\hline 15 & 32768 & $\overline{4914,53}$ & 126,01 & 936,87 & 4915 \\
\hline 16 & 65536 & $\overline{7162,31}$ & 176,32 & 797,67 & 7163 \\
\hline 17 & 131072 & $\overline{17345,01}$ & 250,52 & 1304,04 & 17346 \\
\hline 18 & 262144 & 24315,92 & 279,70 & 1334,72 & 24316 \\
\hline
\end{tabular}

Tabela 4.2: pré-processamento que produz os limiares para a busca subótima em instâncias difíceis.

para cada grupo de instâncias, calculamos a média do tamanho máximo do grafo para o grupo de instâncias de mesmo tamanho e computamos a razão entre o tamanho das instâncias desse grupo e esta média. Nas figuras 4.14(a) e 4.14(b) apresentamos gráficos dessa razão em função do tamanho da instância, para, respectivamente, os experimentos ótimos e sub-ótimos. Os dados utilizados para desenhar estes gráficos são os mesmos apresentados nas tabelas 4.1 e 4.3. Em ambos os experimentos, a razão aumenta em uma taxa uniforme com o incremento do tamanho do experimento, com as exceções de alguns outliers negativos presentes nos experimentos ótimos de tamanho 16 (figura 4.14(a)) e nos experimentos sub-ótimos de tamanhos 15, 17 e 18 (figura 4.14(b)). Portanto, os gráficos sugerem que o uso assintótico de memória mantém uma proporção constante no tamanho da instância.

Para fazermos a análise de alguns aspectos da dinâmica do algoritmo UCS, executamos este algoritmo em grupos de cem instâncias de mesmo tamanho (uma execução por instância). Para cada execução do algoritmo sobre cada instância, armazenamos o número de vezes que é chamada 


\begin{tabular}{|c|c|c|c|c|c|c|c|c|c|c|}
\hline \multicolumn{2}{|c|}{ Instância } & \multicolumn{3}{|c|}{ Tempo (seg) } & \multicolumn{3}{|c|}{ \# Nós computados } & \multicolumn{3}{|c|}{ \# Melhor solução } \\
\hline$\overline{|S|}$ & $2^{|S|}$ & $\mathrm{ES}$ & $\mathrm{UCS}$ & SFFS & $\overline{\mathrm{ES}}$ & $\mathrm{UCS}$ & SFFS & $\overline{\mathrm{ES}}$ & $\mathrm{UCS}$ & SFFS \\
\hline 7 & 128 & 0,14 & 0,18 & 0,14 & 57 & 50,84 & 41,29 & 55 & 86 & 29 \\
\hline 8 & 256 & 0,16 & 0,28 & 0,12 & 93 & 81,95 & 60,33 & 48 & 83 & 20 \\
\hline 9 & 512 & 0,16 & 0,34 & 0,16 & 164 & 126,64 & 91,94 & 46 & 93 & 27 \\
\hline 10 & 1024 & 0,21 & 0,36 & 0,10 & 248 & 194,68 & 142,99 & 40 & 90 & 9 \\
\hline 11 & 2048 & 0,26 & 0,50 & 0,14 & 387 & 314,81 & 203,97 & 34 & 88 & 15 \\
\hline 12 & 4096 & 0,32 & 1,01 & 0,18 & 1022 & 622,34 & 444,34 & 25 & 96 & 14 \\
\hline 13 & 8192 & 0,42 & 2,04 & 0,19 & 1904 & 1142,47 & 700,09 & 32 & 93 & 6 \\
\hline 14 & 16384 & 0,60 & 3,91 & 0,26 & 3492 & 1622,40 & 1122,42 & 20 & 99 & 6 \\
\hline 15 & 32768 & 0,89 & 5,53 & 0,29 & 4915 & 2639,41 & 1 193,18 & 35 & 95 & 10 \\
\hline 16 & 65536 & 1,17 & 8,99 & 0,23 & 7163 & 3699,55 & 1024,84 & 30 & 95 & 13 \\
\hline 17 & 131072 & 2,48 & 26,59 & 0,27 & 17346 & 8556,74 & 1632,69 & 26 & 93 & 5 \\
\hline 18 & 262144 & 3,52 & 61,84 & 0,26 & 24316 & 13743,22 & 1651,80 & 22 & 90 & 1 \\
\hline
\end{tabular}

Tabela 4.3: busca subótima em instâncias difíceis; os limiares para cada teste foram obtidos através do pré-processamento exibido na tabela 4.2.

a sub-rotina DFS (i.e., quantas vezes a execução do algoritmo faz uma busca em profundidade no espaço de busca) e o número de vezes que é chamada a sub-rotina Minimal-Element ou Maximal-Element (i.e., quantas vezes a execução do algoritmo recebe um novo elemento que pode pertencer ao espaço de busca corrente). Finalmente, para cada grupo de instâncias, calculamos as médias do número de chamadas de DFS e de Minimal-Element ou Maximal-Element. Nas figuras 4.15(a) e 4.15(b) apresentamos gráficos dessa razão em função do tamanho da instância para os experimentos ótimos e sub-ótimos, respectivamente. Os dados utilizados para desenhar estes gráficos são os mesmos dos utilizados para produzir as tabelas 4.1 e 4.3. Estes gráficos mostram quantas vezes UCS chama Minimal-Element ou MAXimal-ElEMEnt até que o primeiro receba um novo elemento do espaço de busca corrente, o que é uma condição necessária para chamar a subrotina DFS. Com o crescimento do tamanho das instâncias, UCS precisou realizar mais chamadas de Minimal-Element ou de Maximal-Element até receber um elemento do espaço de busca. Em ambos os gráficos, com o incremento do tamanho das instâncias, a distância entre a linha tracejada (chamadas de Minimal-Element ou Maximal-Element) e a linha sólida (chamadas de DFS) também aumenta. Por exemplo, na figura 4.15(a), para instâncias de tamanho 5, em média mais de $17 \%$ das iterações encontram um novo elemento do espaço de busca corrente; por outro lado, para instâncias de tamanho 18 , em média menos de $2,5 \%$ das iterações encontram um novo elemento do espaço de busca corrente.

\subsection{Comentários}

Mostramos neste capítulo que o algoritmo U-CuRVE, introduzido por Ris e colegas em 2010, apresenta um erro que leva a soluções sub-ótimas. Introduzimos o algoritmo U-CURVE-SEARCH (UCS), um algoritmo ótimo para resolver o problema U-curve. Fornecemos também uma análise de complexidade de tempo do novo algoritmo, assim como realizamos experimentos com o mesmo e fizemos uma análise dos resultados obtidos.

As análises do resultados obtidos nos experimentos indicaram que UCS teve um desempenho melhor do que a busca exaustiva ES no número de nós computados, embora UCS tenha demandado maior tempo computacional. Uma investigação da dinâmica das iterações de UCS apontou que um possível gargalo importante do algoritmo, e que resultaria em um maior consumo de tempo por parte do mesmo, é a busca por um elemento do espaço de busca corrente: como vimos na figura 4.15, o algoritmo precisa chamar várias vezes a sub-rotina MinimaL-ELEMENT ou MAXIMAL-ELEMENT até encontrar um elemento do espaço de busca corrente, problema este que se agrava proporcionalmente 


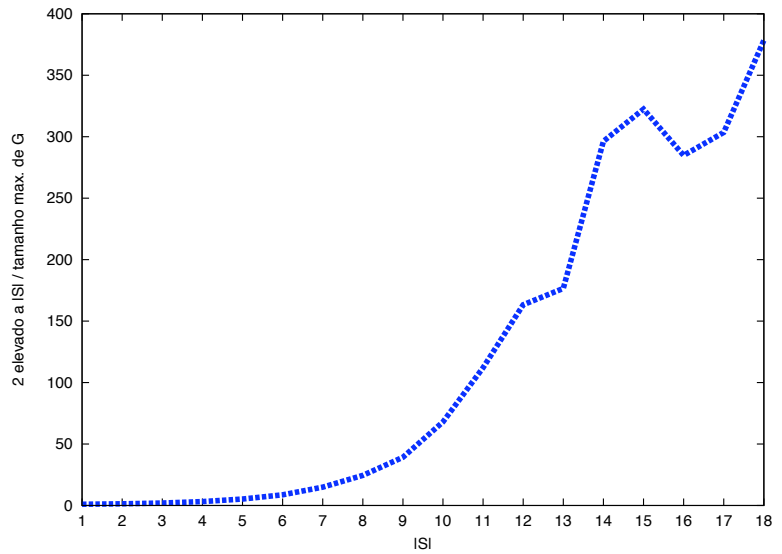

(a)

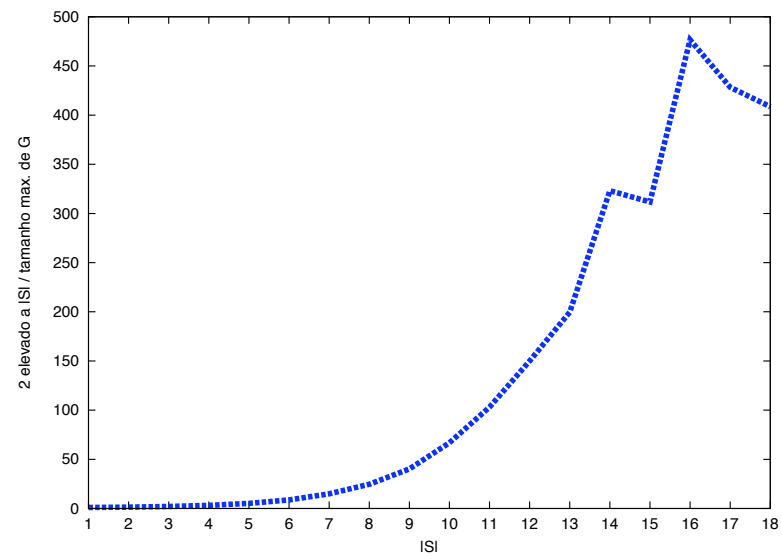

(b)

Figura 4.14: a evolução do tamanho máximo de um grafo $G$ em busca ótima (figura 4.14(a)) e sub-ótima (figura 4.14(b)), durante a execução do algoritmo UCS em instâncias simuladas. Cada linha mostra, para diferentes tamanhos de $S$, a razão entre $2^{|S|}$ (i.e., o tamanho do espaço de busca) e o tamanho máximo do grafo. As linhas vermelha e azul foram desenhadas utilizando os mesmos dados empregados para produzir as tabelas 4.1 e 4.3 , respectivamente.

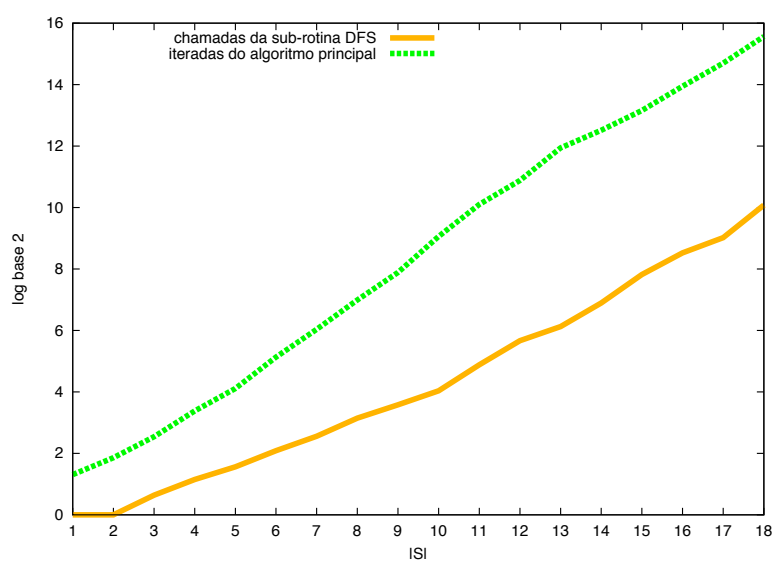

(a)

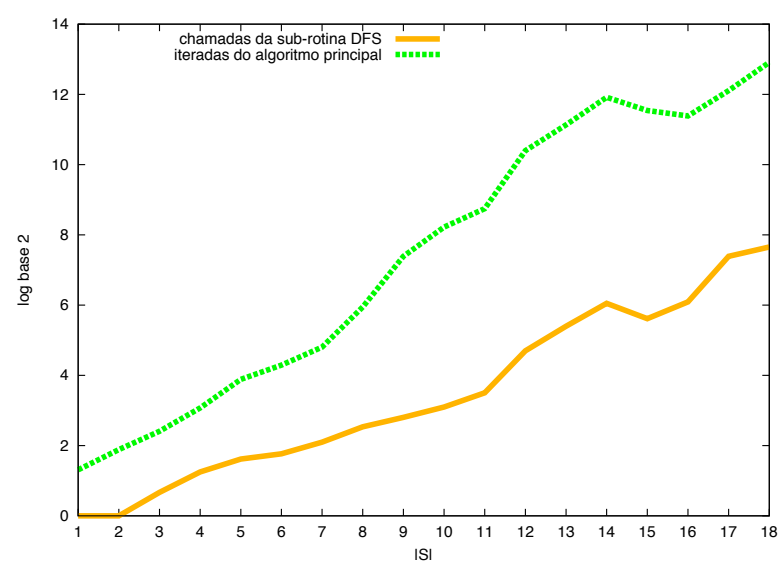

(b)

Figura 4.15: comparação entre o número de vezes que a sub-rotina DFS é chamada (linha sólida) e o número de iterações do algoritmo principal (linha tracejada), durante a execução do algoritmo UCS em instâncias de diferentes tamanhos. Tanto na figura 4.15(a) (experimentos ótimos) quanto na figura 4.15(b) (experimentos sub-ótimos) observamos que para instâncias de tamanho 18 o número de iterações do algoritmo principal é ao menos duas ordens de magnitude maior do que o número de chamadas de DFS. 
ao tamanho da instância em questão.

No capítulo a seguir, abordaremos técnicas para tentar contornar o problema da busca por um elemento do espaço de busca corrente, visando assim tornar o algoritmo UCS mais eficiente em termos de consumo de tempo computacional. 


\section{Capítulo 5}

\section{O problema da busca de início de caminho}

No capítulo anterior, apresentamos U-CURVE-SEARCH (UCS), um algoritmo ótimo para resolver o problema U-curve. As análises do resultados obtidos em experimentos realizados com dados simulados indicaram que UCS demandou um maior tempo computacional do que a busca exaustiva ES, embora UCS tenha computado menos vezes a função custo. Com o objetivo de investigar este fato, uma análise da dinâmica das iterações de UCS apontou que um possível gargalo importante do algoritmo, e que resultaria em um maior consumo de tempo por parte do mesmo, é a busca de um elemento do espaço de busca corrente. Este problema consiste em, dado um espaço de busca corrente $\mathcal{X}\left(\mathcal{R}_{L}, \mathcal{R}_{U}\right)$ definido pelas coleções de restrições inferiores e superiores, respectivamente, $\mathcal{R}_{L}$ e $\mathcal{R}_{U}$, encontrar um elemento de $\mathcal{X}\left(\mathcal{R}_{L}, \mathcal{R}_{U}\right)$ ou então determinar que tal elemento não existe (i.e., que o espaço de busca corrente é vazio). Tal problema também é conhecido como o problema da busca de início de caminho, pois na dinâmica do algoritmo UCS um elemento que pertence ao espaço de busca corrente é necessário para iniciar a etapa da busca em profundidade.

Neste capítulo abordaremos técnicas para tentar contornar o problema da busca de início de caminho, visando assim tornar o algoritmo UCS mais eficiente em termos de consumo de tempo computacional. Iniciaremos formalizando o problema principal e duas variações do mesmo, apresentando também suas respectivas análises de complexidade. Em seguida, apresentaremos duas abordagens para o problema e suas variações: uma que utiliza técnicas de Programação Linear (PL) e outra que faz uso de uma nova estrutura de dados combinada com um método de enumeração. Mostraremos alguns experimentos realizados com as implementações das duas abordagens e faremos uma análise dos resultados obtidos. Finalmente, concluiremos o capítulo discutindo possíveis trabalhos futuros.

\subsection{Formalização do problema e análise de complexidade}

Sejam $S$ um conjunto finito e não-vazio, $\mathcal{R}_{L}$ um conjunto de restrições inferiores, $\mathcal{R}_{U}$ um conjunto de restrições superiores e $\mathcal{X}\left(\mathcal{R}_{L}, \mathcal{R}_{U}\right)$ um espaço de busca corrente do espaço de busca $\mathcal{P}(S)$. Como foi demonstrado na proposição 4.3.1, os tamanhos de $\mathcal{R}_{L}$ e de $\mathcal{R}_{U}$ podem ser exponenciais em $|S|$. Todavia, consideremos o seguinte problema, que aborda somente os casos em que $\mathcal{R}_{L}$ e $\mathcal{R}_{U}$ têm tamanhos polinomiais em $|S|$.

Problema 5.1.1. (problema da busca de início de caminho) Sejam $S$ um conjunto finito e nãovazio, $\mathcal{R}_{L}$ e $\mathcal{R}_{U}$ coleções de restrições, respectivamente, inferiores e superiores, cujos tamanhos são polinomiais em $|S|$. Seja $\mathcal{X}\left(\mathcal{R}_{L}, \mathcal{R}_{U}\right)$ um espaço de busca corrente do espaço de busca $\mathcal{P}(S)$. Encontrar um elemento $X$ de $\mathcal{X}\left(\mathcal{R}_{L}, \mathcal{R}_{U}\right)$.

Caso façamos restrições quanto à cardinalidade do elemento do espaço de busca corrente a ser encontrado, então definimos duas variações do problema 5.1.1.

Problema 5.1.2. (problema da busca de início de caminho mínimo) Sejam $S$ um conjunto finito e não-vazio, $\mathcal{R}_{L}$ e $\mathcal{R}_{U}$ coleções de restrições, respectivamente, inferiores e superiores, cujos tamanhos 
são polinomiais em $|S|$. Seja $\mathcal{X}\left(\mathcal{R}_{L}, \mathcal{R}_{U}\right)$ um espaço de busca corrente do espaço de busca $\mathcal{P}(S)$. Encontrar um elemento $X$ de $\mathcal{X}\left(\mathcal{R}_{L}, \mathcal{R}_{U}\right)$ tal que $|X|$ seja minimo.

Problema 5.1.3. (problema da busca de início de caminho máximo) Sejam $S$ um conjunto finito e não-vazio, $\mathcal{R}_{L}$ e $\mathcal{R}_{U}$ coleções de restrições, respectivamente, inferiores e superiores, cujos tamanhos são polinomiais em $|S|$. Seja $\mathcal{X}\left(\mathcal{R}_{L}, \mathcal{R}_{U}\right)$ um espaço de busca corrente do espaço de busca $\mathcal{P}(S)$. Encontrar um elemento $X$ de $\mathcal{X}\left(\mathcal{R}_{L}, \mathcal{R}_{U}\right)$ tal que $|X|$ seja máximo.

Encontrar um elemento $X$ do espaço de busca corrente tal que $|X|$ seja mínimo (máximo) pode ser mais vantajoso para iniciar uma iteração do algoritmo UCS do que encontrar um $X$ sem tal restrição, uma vez que atualizações das coleções de restrições feitas com elementos mais próximos das extremidades do reticulado Booleano são mais eficientes do que as feitas com elementos mais próximos da cintura do mesmo (i.e., atualizações desse tipo podam uma parcela maior do reticulado Booleano). Tal fato é garantido pelo resultado a seguir.

Proposição 5.1.4. Sejam $S$ um conjunto finito e não-vazio e $X, Y$ elementos de $\mathcal{P}(S)$. Se:

$$
\left|\left(\frac{|S|}{2}\right)-\right| X||>\left|\left(\frac{|S|}{2}\right)-\right| Y||
$$

então vale que:

$$
|[\emptyset, X] \cup[X, S]|>|[\emptyset, Y] \cup[Y, S]| .
$$

Demonstração.

$$
\begin{aligned}
\left|\left(\frac{|S|}{2}\right)-\right| X||>\left|\left(\frac{|S|}{2}\right)-\right| Y|| & \Longrightarrow 2^{|X|}+2^{|S|-|X|}-1>2^{|Y|}+2^{|S|-|Y|}-1 \\
& \Longrightarrow|[\emptyset, X]|+|[\emptyset, S-X]|-1>|[\emptyset, Y]|+|[\emptyset, S-Y]|-1 \\
& \Longrightarrow|[\emptyset, X]|+|[X, S]|-1>|[\emptyset, Y]|+|[Y, S]|-1 \\
& \Longrightarrow|[\emptyset, X] \cup[X, S]|>|[\emptyset, Y] \cup[Y, S]| .
\end{aligned}
$$

A primeira implicação vale porque a equação $2^{|Z|}+2^{|S|-|Z|}-1,0 \leq|Z| \leq|S|$ é simétrica no eixo $|S| / 2$, monotonicamente decrescente no intervalo $[0,|S| / 2]$ e monotonicamente crescente no intervalo $[|S| / 2,|S|]$.

Faremos agora a análise de complexidade dos problemas 5.1.1, 5.1.2 e 5.1.3. Iniciamos apresentando uma hipótese sobre a complexidade do problema 5.1.1.

Conjectura 5.1.5. O problema da busca de início de caminho (problema 5.1.1) é NP-difícil.

Apesar da dificuldade do problema de início de caminho ser ainda uma questão em aberto, a complexidade de suas duas variantes é conhecida: iniciaremos a demonstração das mesmas descrevendo uma formulação do problema 5.1.2 como um problema de decisão.

Problema 5.1.6. Sejam $S$ um conjunto e $k$ um inteiro não-negativo. Sejam $\mathcal{R}_{L}$ e $\mathcal{R}_{U}$, respectivamente, coleções de restrições inferiores e superiores cujos tamanhos são polinomiais em $|S|$. Decidir se existe um elemento $X$ em $\mathcal{X}\left(\mathcal{R}_{L}, \mathcal{R}_{U}\right)$, tal que $|X| \leq k$.

É pouco provável que o problema 5.1.6 tenha uma solução eficiente.

Lema 5.1.7. O problema 5.1.6 é NP-completo.

Demonstração. Inicialmente verificaremos que o problema 5.1.6 pertence a NP. Seja $X$ um elemento de $\mathcal{P}(S)$. Verificar se $|X| \leq k$ é linear no tamanho de $S$, enquanto que para conferir se $X$ é um elemento de $\mathcal{X}\left(\mathcal{R}_{L}, \mathcal{R}_{U}\right)$ é necessário verificar se $X$ não é coberto por $\mathcal{R}_{L}$ nem por $\mathcal{R}_{U}$, o que pode ser feito em $O\left(|S|\left(\left|\mathcal{R}_{L}\right|+\left|\mathcal{R}_{U}\right|\right)\right)$. 
Para demonstrar que o problema 5.1.6 é NP-difícil, mostraremos que o mesmo é ao menos tão difícil quanto o problema de decidir se um grafo $G=(V, E)$ tem uma cobertura com até $k^{\prime}$ vértices, um problema NP-completo [Kar72]. A redução polinomial é construída definindo o conjunto $S$ como uma cópia de $V$ acrescida de um elemento extra, enquanto que tanto a coleção de restrições inferiores $\mathcal{R}_{L}$ quanto a coleção de restrições superiores $\mathcal{R}_{U}$ são definidas como $\{S-e: e \in E\}$ e $k=k^{\prime}$. Note que $|E|<(|V|+1)^{2}=|S|^{2}$ e que portanto os tamanhos de $\mathcal{R}_{L}$ e de $\mathcal{R}_{U}$ são polinomiais em $|S|$, e que para todo $A, B$ em $\mathcal{R}_{L}\left(\mathcal{R}_{U}\right), A \subseteq B$ implica em $A=B$. Na figura 5.1 mostramos um exemplo de construção da redução polinomial.

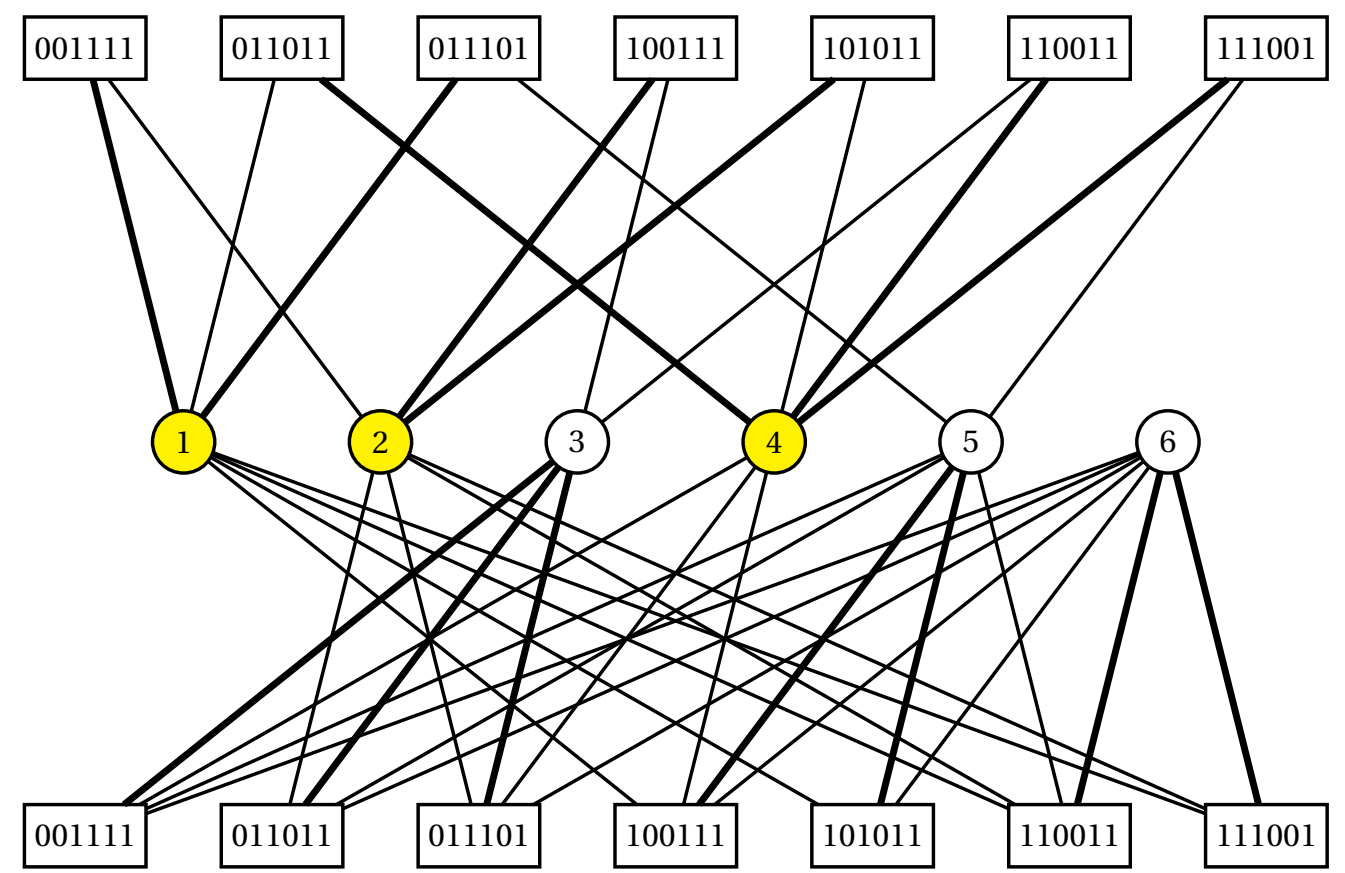

Figura 5.1: exemplo de construção da redução polinomial utilizando como instância do problema da cobertura de vértices o grafo $G$ da figura 2.4. Existe uma cobertura de vértices de $G$ com tamanho até $k^{\prime}$ se e somente se existe um elemento $X$ em $\mathcal{X}\left(\mathcal{R}_{L}, \mathcal{R}_{U}\right)$, tal que $|X| \leq k$.

Observe que se $K$ é uma cobertura de vértices de $G$ com tamanho até $k^{\prime}$, então temos que, para todo elemento $L$ de $\mathcal{R}_{L}$, o subconjunto $X$ de $S$ (definido como $X=K$ ) não está contido em $L$. Além disso, como $X$ não contém o elemento extra em $S$, para todo elemento $U$ de $\mathcal{R}_{U}, X$ não está contido em $U$. Portanto, $X$ é um elemento em $\mathcal{X}\left(\mathcal{R}_{L}, \mathcal{R}_{U}\right)$ que tem cardinalidade menor ou igual a $k=k^{\prime}$. Inversamente, se $X$ é um elemento em $\mathcal{X}\left(\mathcal{R}_{L}, \mathcal{R}_{U}\right)$, tal que $|X| \leq k$, então $K=X$ é uma cobertura por vértices de $G$ com cardinalidade menor ou igual a $k^{\prime}=k$, uma vez que o conjunto $\mathcal{R}_{L}$ foi construído a partir de todas as arestas de $G$.

O lema recém apresentado tem como resultado imediato a dificuldade do problema da busca de início de caminho mínimo.

Teorema 5.1.8. O problema 5.1.2 é NP-difícil.

Demonstração. O fato do problema de decisão 5.1.6 ser NP-completo (lema 5.1.7) implica que o problema 5.1.2, que é um problema de otimização relacionado, é NP-difícil.

O princípio da dualidade também nos fornece a complexidade do problema da busca de início de caminho máximo.

Corolário 5.1.9. O problema 5.1.3 é NP-difícil. 
Demonstração. Aplicando o princípio da dualidade, o resultado do teorema 5.1.8 também vale para o reticulado Booleano $(\mathcal{P}(S), \supseteq)$.

\subsubsection{Busca de início de caminho no algoritmo UCS}

Para resolver o problema da busca de início de caminho (problema 5.1.1), o algoritmo UCS adota a seguinte estratégia: a cada nova iteração, é chamada a sub-rotina Minimal-Element (no caso dual, Maximal-Element) para obter:

- um elemento $M$ minimal (maximal) em $\mathcal{X}\left(\mathcal{R}_{L}, \emptyset\right)\left(\mathcal{X}\left(\emptyset, \mathcal{R}_{U}\right)\right)$ ou;

- $N I L$, caso $\mathcal{X}\left(\mathcal{R}_{L}, \emptyset\right)\left(\mathcal{X}\left(\emptyset, \mathcal{R}_{U}\right)\right)$ seja vazio.

Consideremos a primeira situação: se $M$ é coberto por $\mathcal{R}_{U}\left(\mathcal{R}_{L}\right)$ então ele não pertence a $\mathcal{X}\left(\mathcal{R}_{L}, \mathcal{R}_{U}\right)$ e o mesmo é incluído em $\mathcal{R}_{L}\left(\mathcal{R}_{U}\right)$ - diminuindo assim o tamanho de $\mathcal{X}\left(\mathcal{R}_{L}, \emptyset\right)\left(\mathcal{X}\left(\emptyset, \mathcal{R}_{U}\right)\right)$ - e o algoritmo inicia uma nova iteração; caso contrário, $M$ pertence a $\mathcal{X}\left(\mathcal{R}_{L}, \mathcal{R}_{U}\right)$, ou seja, $M$ é um elemento do espaço de busca corrente. Observe que esta estratégia serve como heurística para resolver o problema 5.1.2 (5.1.3): qualquer elemento minimal (maximal) em $\mathcal{X}\left(\mathcal{R}_{L}, \emptyset\right)\left(\mathcal{X}\left(\emptyset, \mathcal{R}_{U}\right)\right)$ também será minimal (maximal) em $\mathcal{X}\left(\mathcal{R}_{L}, \mathcal{R}_{U}\right)$ e possivelmente terá cardinalidade mínima (máxima). Todavia, esta estratégia também apresenta a desvantagem de precisar visitar $O\left(2^{|S|}\right)$ elementos de $\mathcal{P}(S)$ até encontrar um elemento minimal (maximal) ou comprovar que o espaço de busca corrente $\mathcal{X}\left(\mathcal{R}_{L}, \mathcal{R}_{U}\right)$ é vazio.

As próximas seções discutem duas novas abordagens para resolver o problema da busca de início de caminho e suas duas variações.

\subsection{Abordagem do problema usando Programação Linear Inteira}

Para a leitura desta seção, suporemos que o leitor esteja familiarizado com conceitos básicos de Programação Linear (PL), os quais são apresentados em livros-texto tal como o de Dantzig [Dan98].

Sejam $S$ um conjunto finito e não-vazio, $\mathcal{R}_{L}$ uma coleção de restrições inferiores e $\mathcal{R}_{U}$ uma coleção de restrições superiores. Resolver o problema 5.1.2 (5.1.3) é equivalente a resolver o problema de PL descrito a seguir.

\section{Problema 5.2.1.}

$$
\begin{aligned}
\text { minimizar (maximizar) } & \sum_{i \in S} x_{i}, \\
\text { sujeito a } & \sum_{i \notin R} x_{i} \geq 1, \forall R \in \mathcal{R}_{L}, \\
& \sum_{i \in R} x_{i} \leq|R|-1, \forall R \in \mathcal{R}_{U}, \\
& x_{i} \in\{0,1\}, \forall i \in S .
\end{aligned}
$$

Observe que se uma instância do problema 5.2.1 não for viável, então a instância equivalente do problema 5.1.2 (5.1.3) não tem solução. Caso contrário, uma solução $\mathbf{x}$ é equivalente ao vetor característico de um elemento $X$ de $\mathcal{X}\left(\mathcal{R}_{L}, \mathcal{R}_{U}\right)$ tal que $|X|$ seja mínimo (máximo).

O problema 5.2.1 é um problema de Programação Linear Inteira (PLI), pois os elementos do vetor $\mathbf{x}$ só podem assumir dois valores, 0 ou 1. Dessa forma, para resolver esse problema faz-se necessária a utilização de algum método de resolução PLI. Um exemplo de método é a família de algoritmos do tipo branch-and-cut; esse tipo de algoritmo é descrito no trabalho de Ferreira e Wakabayashi [FW96].

A seguir, apresentaremos um algoritmo para resolver o problema 5.1.2; para isso, o mesmo recebe uma instância do problema 5.1.2, transforma-a em uma instância do problema 5.2.1 e resolve este último utilizando um método branch-and-cut. Por fim, o algoritmo devolve a resposta da seguinte forma: 
- se a instância do problema 5.2.1 for viável, então é devolvido o elemento $X$ cujo vetor característico é equivalente a $\mathbf{x}$;

- caso contrário, é devolvido NIL.

Descrição de algoritmo. Min-BrAnCH-And-Cut recebe um conjunto finito e não-vazio $S$, uma coleção de restrições inferiores $\mathcal{R}_{L}$ e uma coleção de restrições superiores $\mathcal{R}_{U}$. Esta sub-rotina devolve um elemento $A$ de $\mathcal{X}\left(\mathcal{R}_{L}, \mathcal{R}_{U}\right)$ tal que $|A|$ seja mínimo, ou $N I L$, caso $\mathcal{X}\left(\mathcal{R}_{L}, \mathcal{R}_{U}\right)$ seja vazio.

Algoritmo dual. MAX-BRANCH-AND-CUT recebe um conjunto finito e não-vazio $S$, uma coleção de restrições inferiores $\mathcal{R}_{L}$ e uma coleção de restrições superiores $\mathcal{R}_{U}$. Esta sub-rotina devolve um elemento $A$ de $\mathcal{X}\left(\mathcal{R}_{L}, \mathcal{R}_{U}\right)$ tal que $|A|$ seja máximo, ou $N I L$, caso $\mathcal{X}\left(\mathcal{R}_{L}, \mathcal{R}_{U}\right)$ seja vazio.

Utilizaremos estes dois algoritmos como sub-rotinas de uma nova versão do algoritmo UCS, na qual Min-Branch-And-Cut (MAX-Branch-And-Cut) substitui a sub-rotina Minimal-Element (MAXimal-Element) para obter um início de caminho.

Descrição de algoritmo. UCS-2 recebe um conjunto finito e não-vazio $S$ e uma função custo $c$ decomponível em curvas em $\mathrm{U}$, e devolve uma coleção $\mathcal{M} \subseteq \mathcal{P}(S)$ que contém todos os elementos em $\mathcal{P}(S)$ de custo mínimo.

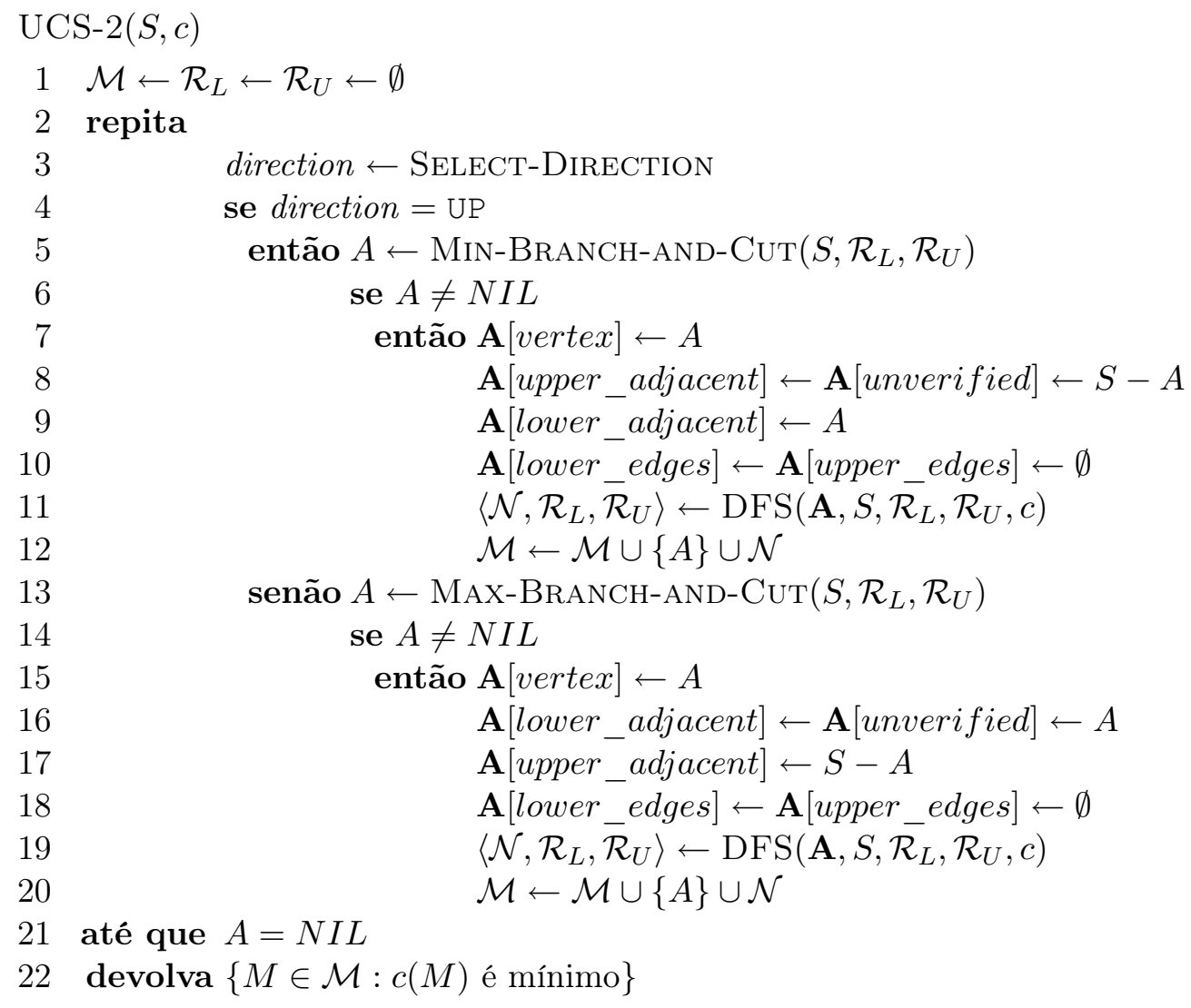

A função SelEct-DiRECTION devolve UP ou DOWN, de acordo com uma distribuição de probabilidade arbitrária.

\subsubsection{Experimentos}

Nesta seção apresentaremos uma avaliação experimental do algoritmo UCS-2. Esta avaliação foi feita empregando o experimento ótimo descrito na seção 2.2.2; este experimento utilizou como 


\begin{tabular}{|c|c|c|c|c|c|}
\hline \multicolumn{2}{|c|}{ Instância } & \multicolumn{2}{|c|}{ Tempo (seg) } & \multicolumn{2}{|c|}{ \# Nós computados } \\
\hline$\overline{|S|}$ & $2^{|S|}$ & UCS-2 & $\mathrm{UCS}$ & UCS-2 & $\mathrm{UCS}$ \\
\hline 1 & 2 & 0,02 & 0,01 & 2,49 & 2,00 \\
\hline 2 & 4 & 0,02 & 0,02 & 4,09 & 3,74 \\
\hline 3 & 8 & 0,01 & 0,01 & 6,77 & 6,67 \\
\hline 4 & 16 & 0,01 & 0,01 & 12,17 & 11,84 \\
\hline 5 & 32 & 0,01 & 0,02 & 22,48 & 22,29 \\
\hline 6 & 64 & 0,03 & 0,02 & 35,90 & 36,03 \\
\hline 7 & 128 & 0,04 & 0,04 & 62,12 & 59,31 \\
\hline 8 & 256 & 0,06 & 0,06 & 96,87 & 95,84 \\
\hline 9 & 512 & 0,13 & 0,12 & 162,17 & 159,60 \\
\hline 10 & 1024 & 0,29 & 0,17 & 262,34 & 255,89 \\
\hline 11 & 2048 & 1,53 & 0,35 & 498,10 & 498,52 \\
\hline 12 & 4096 & 5,89 & 0,70 & 793,28 & 815,94 \\
\hline 13 & 8192 & 24,85 & 1,51 & 1393,92 & 1410,98 \\
\hline 14 & 16384 & 144,26 & 3,25 & 2311,15 & 2278,83 \\
\hline
\end{tabular}

Tabela 5.1: comparação entre UCS e UCS-2 (busca de início de caminho utilizando PL), com este último sempre buscando um início de caminho de cardinalidade minima (máxima). Embora ambos os algoritmos sejam equivalentes do ponto de vista semântico, UCS mostrou-se mais eficiente do ponto de vista computacional.

função custo a equação 3.1, descrita na seção 2.2.2, e como algoritmos, UCS-2 e UCS. Dois experimentos foram realizados: no primeiro deles, a cada chamada do método branch-and-cut, o mesmo é executado até que seja encontrado um conjunto $X$ do espaço de busca corrente tal que $|X|$ seja mínimo (máximo). Já no segundo experimento, o método termina a sua execução assim que é encontrada a primeira solução viável, isto é, o mesmo devolve um conjunto $X$ do espaço de busca corrente, mas sem garantia de minimalidade (maximalidade).

Para realizar estes experimentos, implementamos o algoritmo UCS-2 utilizando o arcabouço featsel. As sub-rotinas Min-Branch-And-Cut e MAX-Branch-And-Cut foram implementadas utilizando a API GNU Linear Programming Toolkit (glpk) [Mak11]; o glpk disponibiliza funções para resolver PLI utilizando um método branch-and-cut [Mak11].

Na tabela 5.1 mostramos os resultados do primeiro experimento. Do ponto de vista semântico, UCS e UCS-2 são equivalentes, com ambos computando um número similar de vezes a função custo. Todavia, do ponto de vista de desempenho computacional, para grupos de instâncias de tamanho a partir de 10, UCS mostrou-se bem mais eficiente que UCS-2.

$\mathrm{Na}$ tabela 5.2 mostramos os resultados do segundo experimento. Do ponto de vista semântico, UCS e UCS-2 seguem sendo equivalentes, com ambos computando um número similar de vezes a função custo. Já do ponto de vista de desempenho computacional, para grupos de instâncias de tamanho a partir de 11, UCS mostrou-se mais eficiente que UCS-2, porém com uma vantagem menor do que a apresentada no primeiro experimento.

Os resultados obtidos nestes dois experimentos mostraram que, para obter um elemento do espaço de busca corrente ao longo da execução do algoritmo UCS, resolver diretamente o problema de início de caminho e suas variações não é eficiente do ponto de vista computacional. Dessa forma, na seção a seguir exploraremos uma nova abordagem, que considera informações fornecidas pela dinâmica do algoritmo UCS.

\subsection{Abordagem do problema usando uma nova estrutura de dados}

No algoritmo UCS original, durante uma busca de início de caminho, dado um elemento $X$ de $\mathcal{P}(S)$ é verificado se o mesmo não é coberto pelas coleções de restrições inferiores e superiores. 


\begin{tabular}{|c|c|c|c|c|c|}
\hline \multicolumn{2}{|c|}{ Instância } & \multicolumn{2}{|c|}{ Tempo (seg) } & \multicolumn{2}{|c|}{ \# Nós computados } \\
\hline$\overline{|S|}$ & $2^{|S|}$ & UCS-2 & UCS & UCS-2 & $\mathrm{UCS}$ \\
\hline 1 & 2 & 0,01 & 0,01 & 2,50 & 2,00 \\
\hline 2 & 4 & 0,01 & 0,01 & 3,99 & 3,77 \\
\hline 3 & 8 & 0,02 & 0,01 & 7,72 & 6,67 \\
\hline 4 & 16 & 0,01 & 0,01 & 13,48 & 12,23 \\
\hline 5 & 32 & 0,01 & 0,01 & 21,37 & 20,65 \\
\hline 6 & 64 & 0,02 & 0,02 & 37,93 & 35,17 \\
\hline 7 & 128 & 0,03 & 0,03 & 60,70 & 59,37 \\
\hline 8 & 256 & 0,07 & 0,06 & 88,59 & 89,97 \\
\hline 9 & 512 & 0,11 & 0,09 & 174,21 & 159,75 \\
\hline 10 & 1024 & 0,29 & 0,21 & 295,49 & 278,27 \\
\hline 11 & 2048 & 0,58 & 0,32 & 432,84 & 436,91 \\
\hline 12 & 4096 & 2,47 & 0,67 & 793,55 & 788,89 \\
\hline 13 & 8192 & 10,28 & 1,44 & 1281,22 & 1257,63 \\
\hline 14 & 16384 & 50,12 & 3,64 & 2373,13 & 2380,17 \\
\hline
\end{tabular}

Tabela 5.2: comparação entre UCS e UCS-2 (busca de início de caminho utilizando PL), com este último buscando um início de caminho qualquer (i.e., devolvendo a primeira solução viável encontrado pelo branch and cut). Apesar de que ambos os algoritmos sejam equivalentes do ponto de vista semântico, UCS mostrouse mais eficiente do ponto de vista computacional, embora a diferença entre eles seja menor do que a apresentada no primeiro experimento.

Tais coleções são armazenadas em listas duplamente encadeadas, nas quais cada restrição ocupa $n$ bits. Todavia, esta busca de início de caminho apresenta dois pontos fracos além dos problemas já citados na seção 5.1.1:

1. a consulta às coleções de restrições é computacionalmente cara;

2. a escolha de $X$ é feita de forma aleatória e pouco sistemática.

Nesta seção, definiremos uma estrutura de dados para armazenar as coleções de restrições, de forma que as consultas às mesmas sejam computacionalmente mais eficientes. Em seguida, mostraremos um resultado que ajuda a restringir a escolha de $X$, tornando o processo mais sistemático. Finalmente, mostraremos um algoritmo para percorrer a estrutura de dados e os resultados de experimentos com o mesmo.

\subsubsection{Estrutura de dados para armazenamento de coleção de restrições}

Apresentaremos agora uma estrutura de dados (ED) mais adequada para verificar se um elemento $X$ de $\mathcal{P}(S)$ é coberto por uma coleção de restrições inferiores $\mathcal{R}_{L}$. Pelo princípio da dualidade, todos os resultados que forem mostrados nesta seção também valem para verificar se $X$ é coberto por uma coleção de restrições superiores $\mathcal{R}_{U}$.

A ED faz uso da constatação da equivalência:

$$
X \in \mathcal{X}\left(\mathcal{R}_{L}, \emptyset\right) \Longleftrightarrow \forall R \in \mathcal{R}_{L}, \exists x \in X: x \notin R
$$

Ou seja, $X$ pertence a $\mathcal{X}\left(\mathcal{R}_{L}, \emptyset\right)$ se e somente se para cada $R$ de $\mathcal{R}_{L}, X$ possui um ou mais elementos que $R$ não possui. Dessa forma, para construirmos $X$ de forma iterativa, precisamos escolher um $X$ que tenha ao menos um de seus elementos em $R^{c}$, para todo $R \in \mathcal{R}_{L}$.

Sejam $R_{1}, R_{2}$ elementos de $\mathcal{R}_{L}$. Se $R_{1}^{c} \cap R_{2}^{c} \neq \emptyset$, então a escolha de um desses elementos em comum para a construção de $X$ nos garante que este último não será coberto por $R_{1}$ nem por $R_{2}$. Por exemplo, se $R_{1}=100$ e $R_{2}=001$, então temos que $R_{1}^{c} \cap R_{2}^{c}=011 \cap 110=010$, o que implica que após a operação $X \leftarrow X \cup 010$ o elemento $X$ não será coberto por $R_{1}$ nem por $R_{2}$. 
Portanto, iremos utilizar a nova ED para armazenar os elementos de $R^{c}, R \in \mathcal{R}_{L}$. A ED faz tal armazenamento utilizando uma matriz $A[1, \ldots,|S|, 1 \ldots 3]$, organizada da seguinte forma:

- $A[1, \ldots,|S|, 1]$ é um vetor dos índices de uma bijeção $f$ dos elementos de $S$ para $\{1 \ldots|S|\}$;

- $A[1, \ldots,|S|, 2]$ é um vetor de coleções de elementos de $\mathcal{R}_{L}$. Se $A[i, 2]$ contém um elemento $R$, então $R^{c}$ contém o elemento $f(A[i, 1])$;

- $A[1, \ldots,|S|, 3]$ é um vetor de inteiros não-negativos. $A[i, 3]$ representa o tamanho da coleção $A[i, 2]$.

Utilizando um método de ordenação estável, podemos ordenar de forma não-crescente as linhas de $A$ pelos tamanhos das coleções da coluna 2. Dessa forma, a primeira linha contém uma maior coleção de elementos de $\mathcal{R}_{L}$ tal que para cada $R$ dessa coleção, vale que $f(1)$ pertence a $R^{c}$. A figura 5.2 contém um exemplo de aplicação da nova ED para um reticulado Booleano de grau 5. A figura 5.2(a) mostra um espaço de busca corrente $\mathcal{X}\left(\mathcal{R}_{L}, \emptyset\right)$ definido pela coleção de restrições inferiores $\mathcal{R}_{L}=\{11000,01110,00111,01011\}$, enquanto que a figura 5.2(b) ilustra a nova ED armazenando estas quatro restrições; observe que uma ordenação estável não-crescente foi aplicada na ED, o que implica que a coleção de elementos da primeira linha tem cardinalidade máxima.

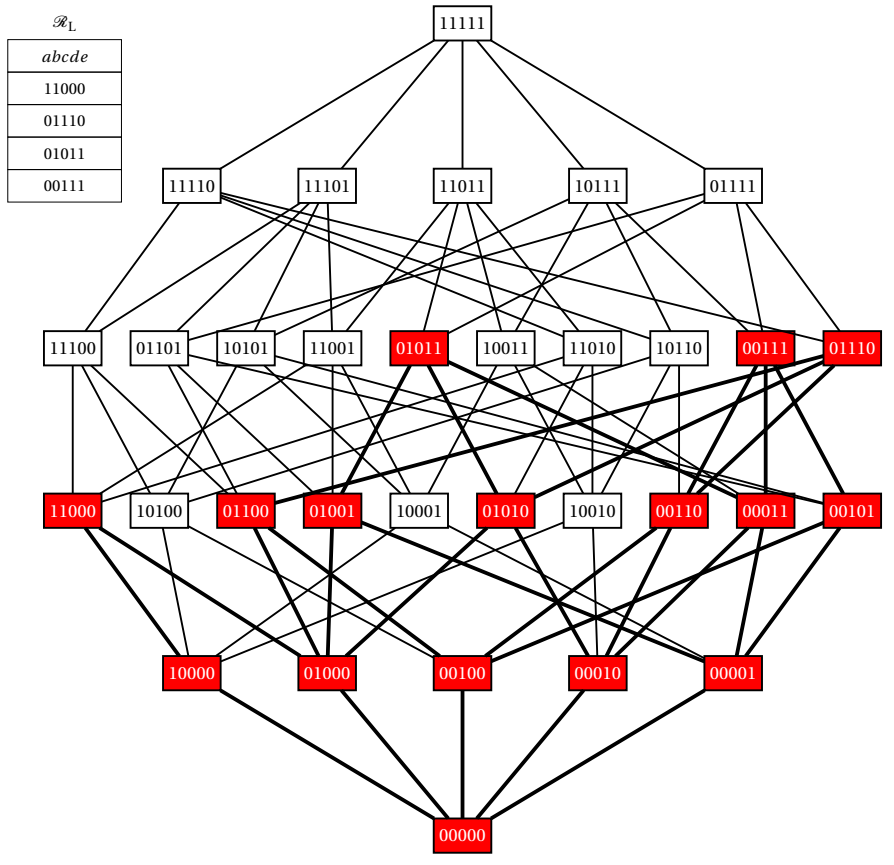

(a)

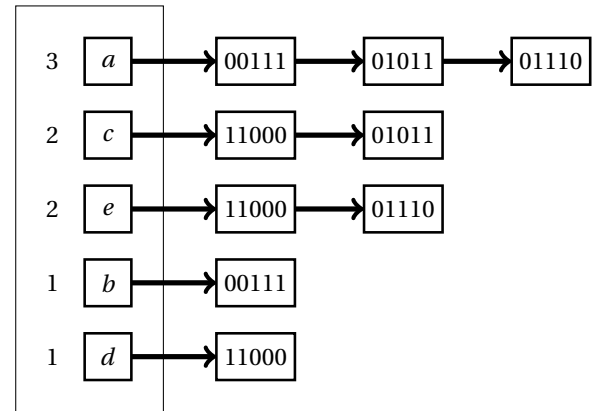

(b)

Figura 5.2: um exemplo de aplicação da nova ED para uma instância de 5 elementos. A figura 5.2(a) mostra um espaço de busca corrente $\mathcal{X}\left(\mathcal{R}_{L}, \emptyset\right)$ definido pela coleção de restrições inferiores $\mathcal{R}_{L}=$ $\{11000,01110,00111,01011\}$, enquanto que a figura 5.2(b) ilustra a nova ED armazenando estas quatro restrições; observe que a ordenação estável foi aplicada na ED, e que portanto a coleção de elementos da primeira linha tem cardinalidade máxima.

A razão para ordenarmos as linhas da ED, de forma estável e não-crescente e utilizando os tamanhos das coleções de elementos de $\mathcal{R}_{L}$ como chaves, é que trabalhamos com a hipótese de que assim é possível encontrar um elemento de $\mathcal{X}\left(\mathcal{R}_{L}, \emptyset\right)$ visitando menos linhas da ED. Por exemplo, no caso do exemplo da figura 5.2, se visitarmos as linhas da ED de cima para baixo, então logo na segunda linha temos que $X=10100$ é um elemento de $\mathcal{X}\left(\mathcal{R}_{L}, \emptyset\right)$; porém, se visitarmos as linhas da ED de baixo para cima, somente após a quarta linha é que $X$ será igual a 01111 e pertencerá a $\mathcal{X}\left(\mathcal{R}_{L}, \emptyset\right)$. 
Selecionar primeiramente as linhas com as maiores coleções de elementos de $\mathcal{R}_{L}$ é uma escolha gulosa que funciona diretamente quando queremos encontrar um elemento de $\mathcal{X}\left(\mathcal{R}_{L}, \emptyset\right)$. Porém, o mesmo não é verdade quando estamos resolvendo o problema 5.1.1, ou seja, quando queremos encontrar um elemento de $\mathcal{X}\left(\mathcal{R}_{L}, \mathcal{R}_{U}\right)$ : por exemplo, se temos um reticulado Booleano de grau 5, com $\mathcal{R}_{L}$ definida igual ao exemplo da figura 5.2 e $\mathcal{R}_{U}=10100$, então $X=10100$ não é um elemento de $\mathcal{X}\left(\mathcal{R}_{L}, \mathcal{R}_{U}\right)$ (Figura 5.3). Portanto, um método de enumeração das linhas da ED faz-se necessário para encontrarmos um início de caminho.

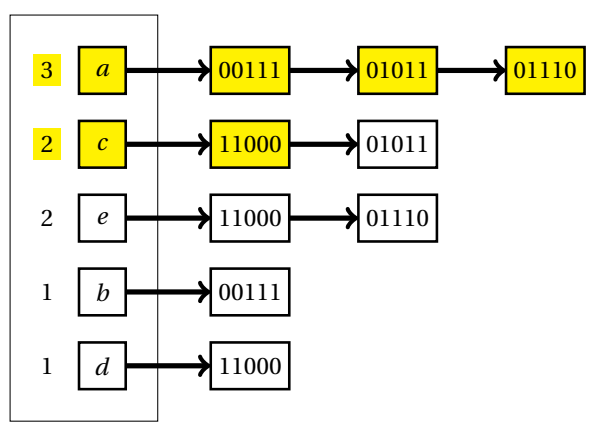

(a)

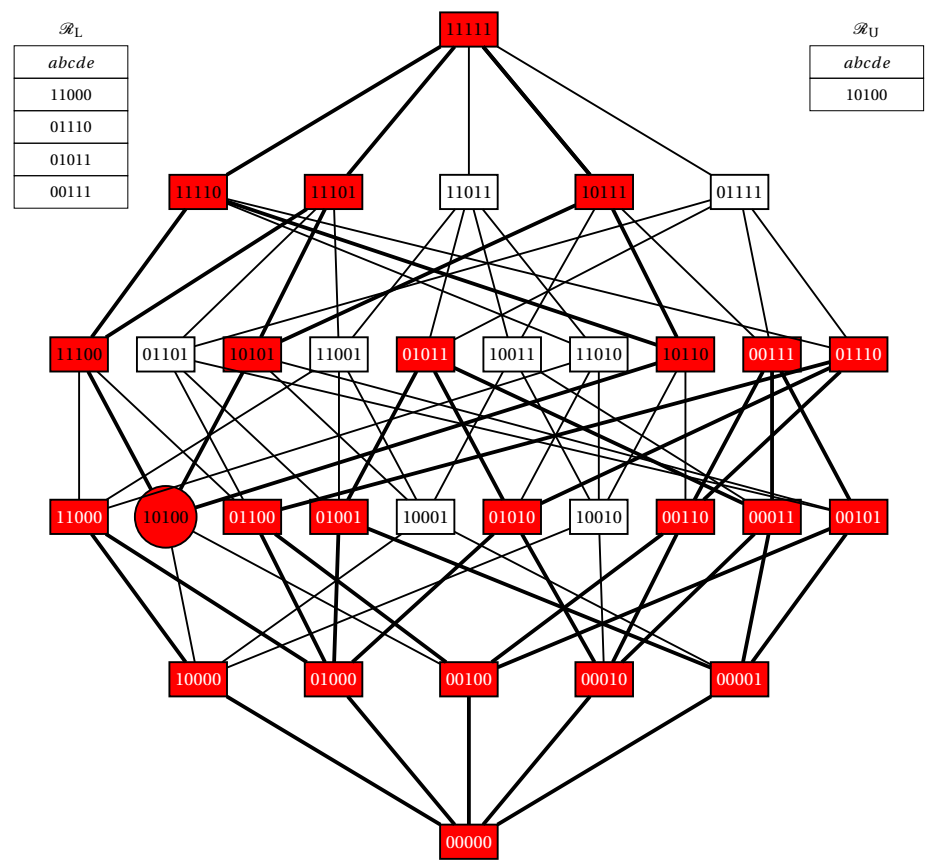

(b)

Figura 5.3: um contra-exemplo de que a escolha gulosa das linhas da ED com as maiores coleções de elementos de $\mathcal{R}_{L}$ não serve para resolver o problema da busca de início de caminho. Escolhendo as duas primeiras linhas (figura 5.3(a)), temos que $X=10100$ não pertence a $\mathcal{X}\left(\mathcal{R}_{L}, \mathcal{R}_{U}\right)$ (figura 5.3(b)).

O método de enumeração que empregamos aqui é o mesmo utilizado pelo algoritmo UBB, que será apresentado no capítulo 6 . Tal enumeração produz uma árvore $T$, que é construída implicitamente. $T$ é um sub-grafo do diagrama de Hasse do reticulado Booleano $(\mathcal{P}(S), \subseteq)$. A construção de $T$ utiliza aplicações recursivas da decomposição do espaço de busca que será apresentada no lema 6.1.1. A figura 5.4 é mostrado um exemplo de aplicação deste esquema de enumeração para a ED do exemplo da figura 5.3. Observe que, nesta enumeração, os elementos são visitados seguindo a ordem das linhas da ED após a ordenação da mesma.

O percorrimento de $T$ pode ser feito de diversas maneiras; dentre elas, há três métodos:

(i) de forma aleatória (i.e., a busca ramifica a partir de um nó qualquer de $T$ );

Esta abordagem garante uma maior aleatoriedade na busca por um início de caminho.

(ii) da esquerda para direita (i.e., a busca ramifica primeiro a partir dos nós cujos elementos são as primeiras linhas da ED ordenada);

A vantagem desta abordagem é de, na prática, encontrar mais rapidamente um elemento que não seja coberto por $\mathcal{R}_{L}$.

(iii) da direita para esquerda (i.e., a busca ramifica primeiro a partir dos nós cujos elementos são as últimas linhas da ED ordenada). 


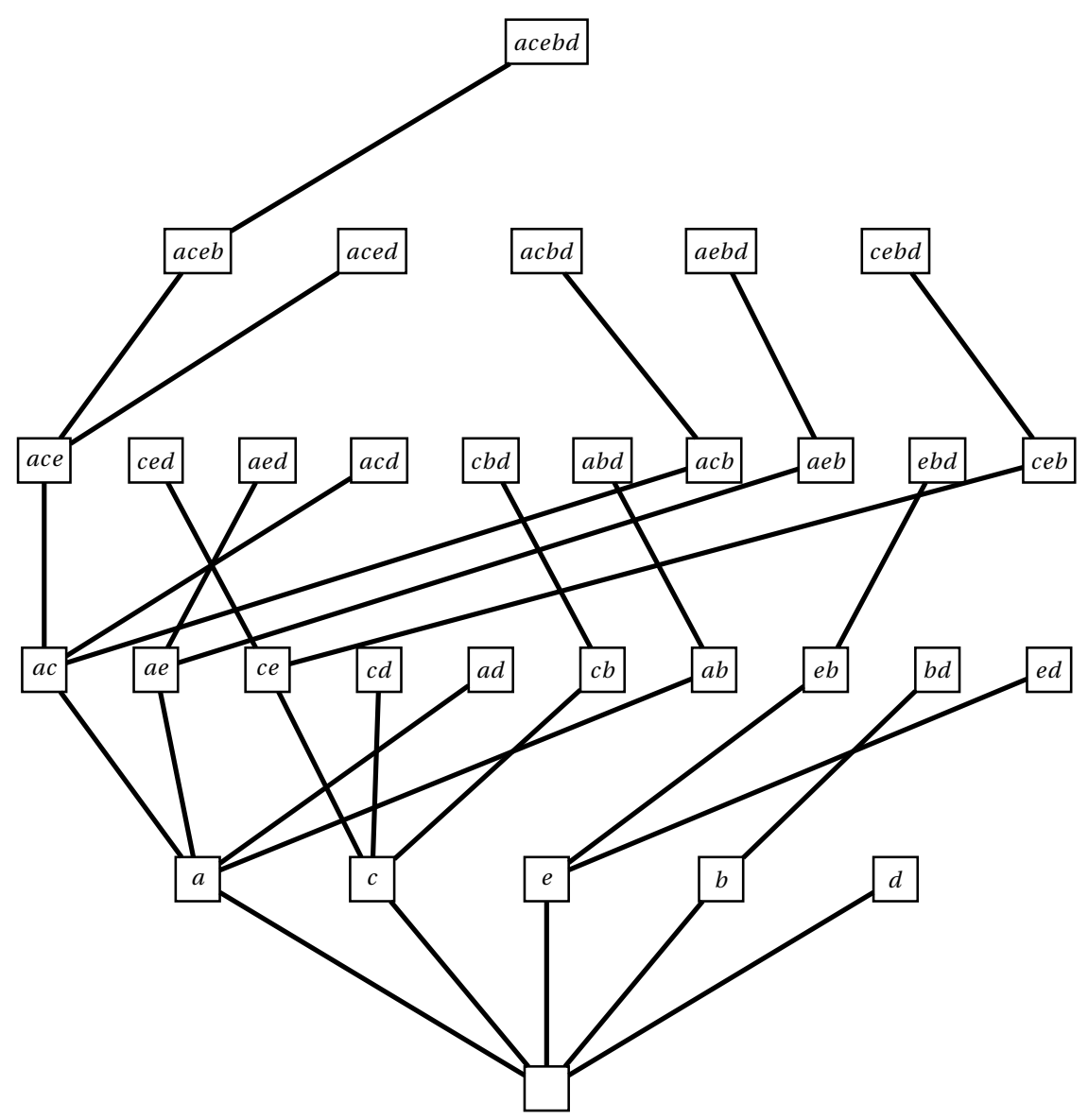

Figura 5.4: árvore de busca $T$ produzida através de uma enumeração dos elementos do exemplo da figura 5.2(b). T foi construída através de aplicações recursivas da decomposição do espaço de busca que é apresentada no lema 6.1.1.

Esta abordagem garante que o primeiro elemento encontrado que não for coberto por $\mathcal{R}_{L}$ seja minimal em $\mathcal{X}\left(\mathcal{R}_{L}, \emptyset\right)$.

Na figura 5.5 apresentamos um exemplo de percorrimento de $T$ de forma aleatória (figura 5.5(a)) e que encontra um elemento do espaço de busca corrente (figura 5.5(b)). Na próxima subseção apresentaremos um algoritmo de percorrimento de $T$ que se utiliza do método (ii).

\subsubsection{Algoritmo de busca de início de caminho}

Iniciaremos mostrando um resultado sobre o comportamento do espaço de busca corrente nas iterações do algoritmo UCS. Aproveitaremos tal resultado para minimizar a necessidade de consulta das coleções de restrições durante o percorrimento da árvore de busca.

Proposição 5.3.1. Sejam $\mathcal{R}_{L}$ uma coleção de restrições inferiores e $\mathcal{R}_{U}$ uma coleção de restrições superiores. Seja $M_{i}$ um elemento do espaço de busca corrente da iteração $i$ de uma execução do algoritmo UCS, tal que $\left|M_{i}\right|$ seja minimo. Se $M_{i+1}$ é um elemento do espaço de busca corrente da iteração $i+1$ da mesma execução e do mesmo algoritmo, tal que $\left|M_{i+1}\right|$ seja mínimo, então vale que $\left|M_{i}\right| \leq\left|M_{i+1}\right|$.

Demonstração. Pela correção do algoritmo UCS, elementos de $\mathcal{P}(S)$ que são removidos do espaço de busca corrente não são adicionados novamente em iterações posteriores. Portanto, se $M_{i}$ é um elemento do espaço de busca corrente da iteração $i$ de uma execução do algoritmo UCS, tal que $\left|M_{i}\right|$ seja mínimo, então na iterações posteriores a menor cardinalidade possível para qualquer elemento 


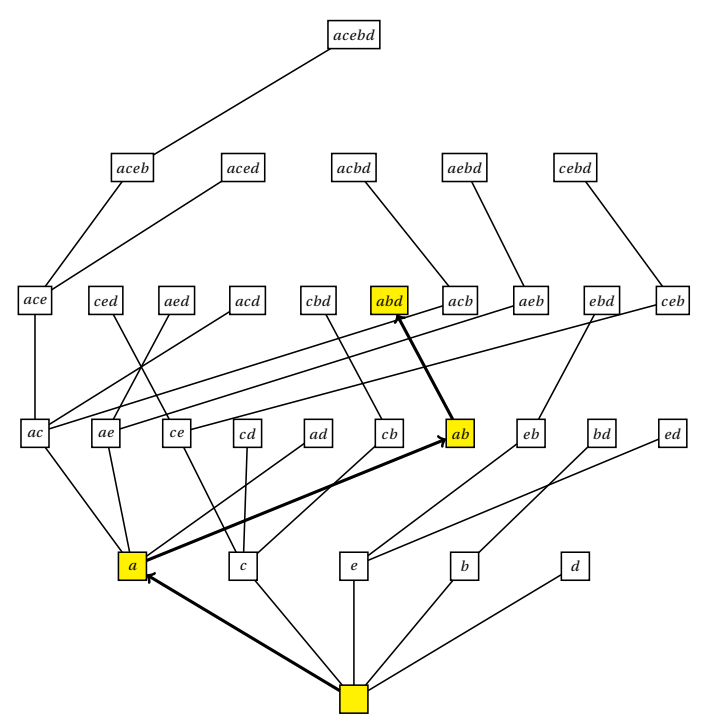

(a)

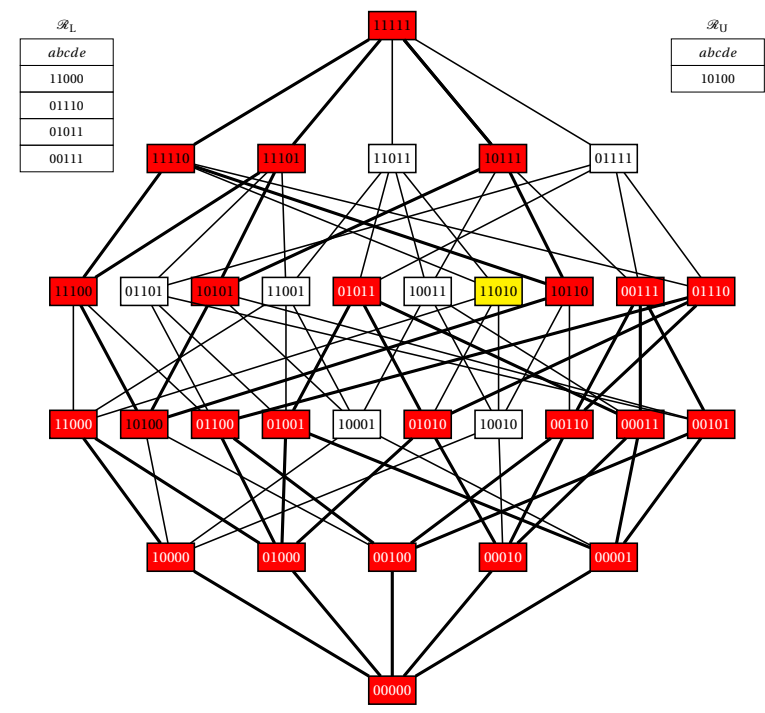

(b)

Figura 5.5: um exemplo de percorrimento de uma árvore de busca $T$, visando encontrar um início de caminho. Figura 5.5(a): um percorrimento de T até encontrar o elemento abd =11010; este elemento cobre todos as restrições na ED da figura 5.2(b), portanto ele não é coberto por $\mathcal{R}_{L}$. Figura 5.5(b): verificamos que 11010 pertence ao espaço de busca corrente, pois este elemento não é coberto por $\mathcal{R}_{U}$.

do espaço de busca corrente é $\left|M_{i}\right|$. Logo, se $M_{i+1}$ é um elemento do espaço de busca corrente da iteração $i+1$ da mesma execução e do mesmo algoritmo, tal que $\left|M_{i+1}\right|$ seja mínimo, então vale que $\left|M_{i}\right| \leq\left|M_{i+1}\right|$.

O resultado da proposição 5.3.1 também vale para o reticulado Booleano $(\mathcal{P}(S), \supseteq)$.

Proposição 5.3.2. Sejam $\mathcal{R}_{L}$ uma coleção de restrições inferiores e $\mathcal{R}_{U}$ uma coleção de restrições superiores. Seja $M_{i}$ um elemento do espaço de busca corrente da iteração $i$ de uma execução do algoritmo UCS, tal que $\left|M_{i}\right|$ seja máximo. Se $M_{i+1}$ é um elemento do espaço de busca corrente da iteração $i+1$ da mesma execução e do mesmo algoritmo, tal que $\left|M_{i+1}\right|$ seja máximo, então vale que $\left|M_{i}\right| \geq\left|M_{i+1}\right|$.

Demonstração. Aplicando o princípio da dualidade, o resultado da proposição 5.3.1 também vale para o reticulado Booleano $(\mathcal{P}(S), \supseteq)$.

A proposição 5.3 .1 (5.3.2) diz respeito ao número mínimo (máximo) de elementos de $S$ que uma solução precisa conter, evitando-se enumerações desnecessárias durante a busca de um início de caminho. Um limite inferior (superior) para a cardinalidade de um elemento mínimo (máximo) pode ser obtida através de uma versão relaxada do problema 5.2.1.

\section{Problema 5.3.3.}

$$
\begin{aligned}
\text { minimizar (maximizar) } & \sum_{i \in S} x_{i}, \\
\text { sujeito a } & \sum_{i \notin R} x_{i} \geq 1, \forall R \in \mathcal{R}_{L}, \\
& \sum_{i \in R} x_{i} \leq|R|-1, \forall R \in \mathcal{R}_{U}, \\
& x_{i} \in[0,1], \forall i \in S .
\end{aligned}
$$


As proposições a seguir nos garantem que resolver o problema 5.3.3 de fato fornece um limite inferior (superior) para a cardinalidade de um elemento mínimo (máximo).

Proposição 5.3.4. Sejam $\mathcal{R}_{L}$ uma coleção de restrições inferiores e $\mathcal{R}_{U}$ uma coleção de restrições superiores, tais que $\mathcal{X}\left(\mathcal{R}_{L}, \mathcal{R}_{U}\right) \neq \emptyset$. Seja $X$ um elemento de $\mathcal{X}\left(\mathcal{R}_{L}, \mathcal{R}_{U}\right)$ tal que $|X|$ seja minimo. Se $\mathbf{x}$ é um vetor obtido ao resolver o problema 5.3 .3 utilizando $\mathcal{R}_{L}$ e $\mathcal{R}_{U}$ como restrições, então vale que $\sum_{x \in \mathbf{x}} x \leq|X|$.

Demonstração. Suponhamos que $\sum_{x \in \mathbf{x}} x>|X|$ : neste caso, poderíamos definir um vetor $\mathbf{y}$ como

$$
y_{i}= \begin{cases}1, & \text { se } i \in X \\ 0, & \text { caso contrário. }\end{cases}
$$

y é uma solução viável do problema 5.3.3. Porém, $\sum_{y \in \mathbf{y}} y=|X|<\sum_{x \in \mathbf{x}} x$, o que é uma contradição com a condição de $\mathbf{x}$ ser um vetor obtido ao resolver o problema 5.3.3 utilizando $\mathcal{R}_{L}$ e $\mathcal{R}_{U}$ como restrições. Portanto, vale que $\sum_{x \in \mathbf{x}} x \leq|X|$.

O resultado da proposição 5.3.4 também vale para o reticulado Booleano $(\mathcal{P}(S), \supseteq)$.

Proposição 5.3.5. Sejam $\mathcal{R}_{L}$ uma coleção de restrições inferiores e $\mathcal{R}_{U}$ uma coleção de restrições superiores, tais que $\mathcal{X}\left(\mathcal{R}_{L}, \mathcal{R}_{U}\right) \neq \emptyset$. Seja $X$ um elemento de $\mathcal{X}\left(\mathcal{R}_{L}, \mathcal{R}_{U}\right)$ tal que $|X|$ seja máximo. Se $\mathbf{x}$ é um vetor obtido ao resolver o problema 5.3 .3 utilizando $\mathcal{R}_{L}$ e $\mathcal{R}_{U}$ como restrições, então vale que $\sum_{x \in \mathbf{x}} x \geq|X|$.

Demonstração. Aplicando o princípio da dualidade, o resultado da proposição 5.3.4 também vale para o reticulado Booleano $(\mathcal{P}(S), \supseteq)$.

No problema 5.3.3 não há a exigência de que os elementos do vetor x sejam inteiros. Portanto, é possível utilizar o algoritmo Simplex para estimar o limite inferior (superior) [Dan98]. A seguir, apresentaremos duas sub-rotinas que fazem exatamente estas estimações.

Descrição de algoritmo. Min-Simplex recebe um conjunto finito e não-vazio $S$, uma coleção de restrições inferiores $\mathcal{R}_{L}$, uma coleção de restrições superiores $\mathcal{R}_{U}$. Esta sub-rotina executa o método Simplex, resolvendo o problema de minimização 5.3.3 utilizando $\mathcal{R}_{L}$ e $\mathcal{R}_{U}$ como restrições e obtendo o vetor $\mathbf{x}$ como resposta. Por fim, esta sub-rotina devolve um limite inferior $\left\lceil\sum_{x \in \mathbf{x}} x\right\rceil$ para a cardinalidade de um elemento $X$ de $\mathcal{X}\left(\mathcal{R}_{L}, \mathcal{R}_{U}\right)$ tal que $|X|$ seja mínimo.

Algoritmo dual. MAX-Simplex recebe um conjunto finito e não-vazio $S$, uma coleção de restrições inferiores $\mathcal{R}_{L}$, uma coleção de restrições superiores $\mathcal{R}_{U}$. Esta sub-rotina executa o método Simplex, resolvendo o problema de maximização 5.3.3 utilizando $\mathcal{R}_{L}$ e $\mathcal{R}_{U}$ como restrições e obtendo o vetor $\mathbf{x}$ como resposta. Por fim, esta sub-rotina devolve um limite superior $\left\lfloor\sum_{x \in \mathbf{x}} x\right\rfloor$ para a cardinalidade de um elemento $X$ de $\mathcal{X}\left(\mathcal{R}_{L}, \mathcal{R}_{U}\right)$ tal que $|X|$ seja máximo.

Supondo a obtenção das estimativas de limites superiores e inferiores para o tamanho de um elemento do espaço de busca corrente, apresentaremos a seguir uma sub-rotina e um algoritmo para realizar a busca de um início de caminho; ambos utilizam a estrutura de dados apresentada na figura 5.2(b) para armazenar uma das coleções de restrições, assim como a enumeração mostrada na figura 5.4 para realizar a busca propriamente dita de um início de caminho.

Descrição de algoritmo. LOWER-ElEMENT recebe um conjunto finito e não-vazio $S$, uma coleção de restrições inferiores $\mathcal{R}_{L}$, uma coleção de restrições superiores $\mathcal{R}_{U}$ e um inteiro não-negativo l. Esta sub-rotina devolve um elemento $A$ de $\mathcal{X}\left(\mathcal{R}_{L}, \mathcal{R}_{U}\right)$ tal que $|A| \geq l$, ou $N I L$, caso $\mathcal{X}\left(\mathcal{R}_{L}, \mathcal{R}_{U}\right)$ seja vazio. 


\section{Lower-Element $\left(S, \mathcal{R}_{L}, \mathcal{R}_{U}, l\right)$}

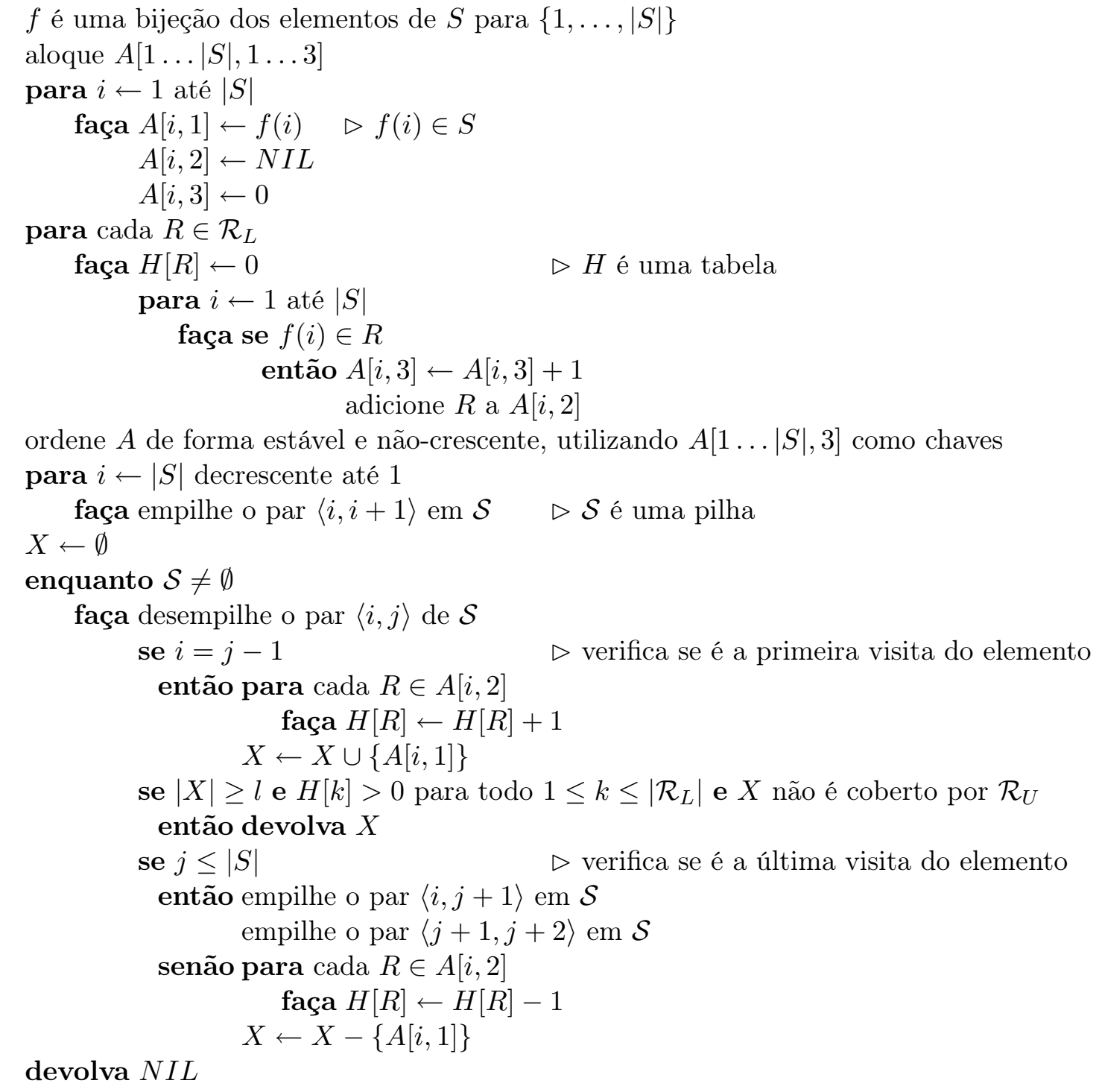

Algoritmo dual. UpPer-Element recebe um conjunto finito e não-vazio $S$, uma coleção de restrições inferiores $\mathcal{R}_{L}$, uma coleção de restrições superiores $\mathcal{R}_{U}$ e um inteiro não-negativo $u$. Esta sub-rotina devolve um elemento $A$ de $\mathcal{X}\left(\mathcal{R}_{L}, \mathcal{R}_{U}\right)$ tal que $|A| \leq u$, ou $N I L$, caso $\mathcal{X}\left(\mathcal{R}_{L}, \mathcal{R}_{U}\right)$ seja vazio.

Finalmente, podemos definir uma nova versão do algoritmo UCS, utilizando as sub-rotinas Min-Simplex e Lower-Element (ou MaX-Simplex e Upper-Element) para obter um início de caminho.

Descrição de algoritmo. UCS-3 recebe um conjunto finito e não-vazio $S$ e uma função custo $c$ decomponível em curvas em $\mathrm{U}$, e devolve uma coleção $\mathcal{M} \subseteq \mathcal{P}(S)$ que contém todos os elementos em $\mathcal{P}(S)$ de custo mínimo. 


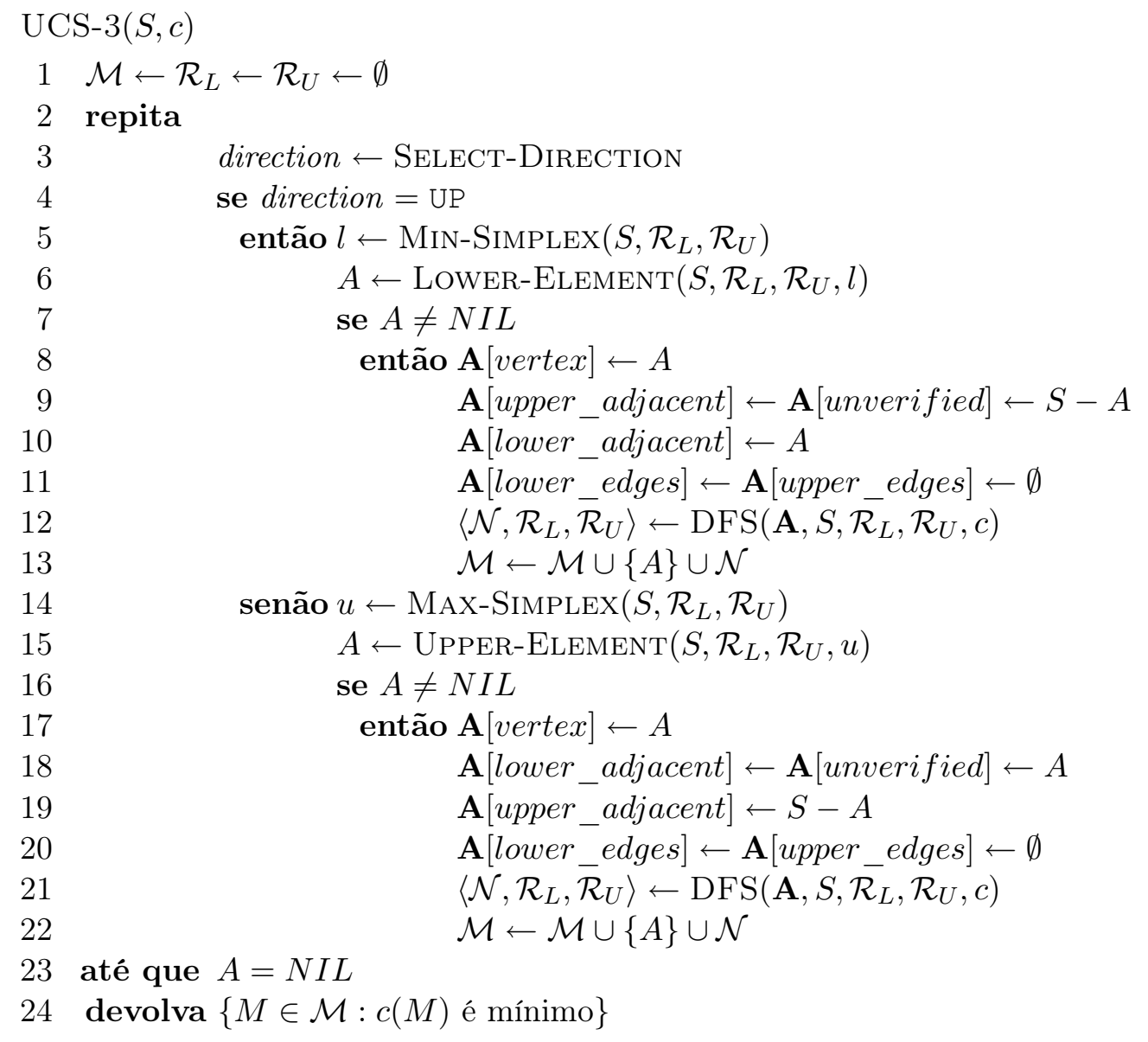

A função SELECT-DiRection devolve UP ou DOWN, de acordo com uma distribuição de probabilidade arbitrária.

\subsubsection{Experimentos}

Nesta seção apresentaremos uma avaliação experimental do algoritmo UCS-3. Esta avaliação foi feita através de um experimento ótimo, conforme descrito em 2.2.2; este experimento utilizou como função custo a equação 3.1, descrita na seção 2.2.2, e como algoritmos, UCS-3 e UCS.

Para realizar estes experimentos, implementamos o algoritmo UCS-3 utilizando o arcabouço featsel. As sub-rotinas Min-Simplex e MAX-Simplex foram implementadas utilizando a API do método Simplex implementado no glpk.

Na tabela 5.3 mostramos os resultados do experimento. Do ponto de vista semântico, UCS e UCS-3 são equivalentes, com ambos computando um número similar de vezes a função custo. Todavia, do ponto de vista de desempenho computacional, para grupos de instâncias de tamanho a partir de 15, UCS mostrou-se mais eficiente que UCS-3. Trata-se de uma melhoria em relação ao UCS-2, pois este último mostrou-se menos eficiente que o UCS para grupos de instâncias a de tamanho a partir de 11 (tabela 5.2).

\subsection{Discussão}

Neste capítulo apresentamos o problema da busca de início de caminho (problema 5.1.1), provando que o mesmo é NP-difícil quando se adiciona a restrição de que o início de caminho seja um elemento mínimo (máximo) do espaço de busca corrente. Apresentamos duas abordagens para o problema: na primeira modelamos o problema como um problema de Programação Linear Inteira, resolvendo-o utilizando um método branch-and-cut; já na segunda, desenvolvemos uma nova estrutura de dados para computar os elementos candidatos a início de caminho, combinada com 


\begin{tabular}{|c|c|c|c|c|c|}
\hline \multicolumn{2}{|c|}{ Instância } & \multicolumn{2}{|c|}{ Tempo (seg) } & \multicolumn{2}{|c|}{ \# Nós computados } \\
\hline$|S|$ & $2^{|S|}$ & UCS-3 & UCS & UCS-3 & UCS \\
\hline 1 & 2 & 0,01 & 0,01 & 2,00 & 2,00 \\
\hline 2 & 4 & 0,01 & 0,01 & 3,79 & 3,80 \\
\hline 3 & 8 & 0,01 & 0,01 & 7,03 & 7,03 \\
\hline 4 & 16 & 0,01 & 0,01 & 12,30 & 12,33 \\
\hline 5 & 32 & 0,01 & 0,02 & 20,95 & 20,96 \\
\hline 6 & 64 & 0,02 & 0,02 & 34,32 & 34,28 \\
\hline 7 & 128 & 0,03 & 0,03 & 53,85 & 54,23 \\
\hline 8 & 256 & 0,05 & 0,06 & 105,12 & 103,73 \\
\hline 9 & 512 & 0,08 & 0,10 & 156,41 & 156,31 \\
\hline 10 & 1024 & 0,15 & 0,17 & 273,13 & 271,88 \\
\hline 11 & 2048 & 0,30 & 0,39 & 455,11 & 452,58 \\
\hline 12 & 4096 & 0,68 & 0,65 & 810,81 & 804,33 \\
\hline 13 & 8192 & 1,57 & 1,34 & 1158,81 & 1177,28 \\
\hline 14 & 16384 & 4,16 & 3,02 & 1999,61 & 2002,83 \\
\hline 15 & 32768 & 16,43 & 8,70 & 4042,23 & 4051,17 \\
\hline 16 & 65536 & 59,63 & 27,71 & 7670,00 & 7649,81 \\
\hline
\end{tabular}

Tabela 5.3: comparação entre UCS e UCS-3 (busca de início de caminho utilizando uma nova estrutura de dados e um novo algoritmo de busca). Ambos os algoritmos são equivalentes do ponto de vista semântico, mas UCS mostrou-se mais eficiente do ponto de vista computacional a partir de instâncias de tamanho 15.

um algoritmo de busca que faz uso de uma enumeração do espaço de busca e, utilizando o método Simplex, delimitando as cardinalidades mínimas e máximas que um elemento do espaço de busca corrente poderia ter.

A segunda abordagem, implementada no algoritmo UCS-3, ainda apresenta uma vantagem sobre a primeira abordagem (UCS-2) e também sobre a heurística do UCS original: graças à enumeração do espaço de busca, a busca de início de caminho do algoritmo UCS-3 é feita de maneira mais sistemática, portanto a mesma é facilmente paralelizável.

Não obstante, os resultados experimentais mostraram que, apesar de UCS-3 ser computacionalmente mais eficiente que UCS-2, UCS-3 ainda perde em consumo de tempo para a heurística utilizada pelo algoritmo UCS original para encontrar um início de caminho. Portanto, o problema da busca de início de caminho segue sendo um problema em aberto. Além de responder se o problema é NP-difícil (conjectura 5.1.5), também é necessário definir novas estratégias para resolvê-lo. A seguir, listaremos três possibilidades de avanço nesta linha de pesquisa.

\subsubsection{Múltiplas buscas em profundidade}

O objetivo desta abordagem é minimizar, durante a execução do algoritmo UCS ou de uma de suas variantes, o número de chamadas de uma busca de início de caminho. Tal minimização é desejável, pois uma busca de início de caminho é um procedimento computacionalmente caro em quaisquer uma de suas abordagens.

Uma análise do comportamento da sub-rotina DFS durante a execução do algoritmo UCS-3 nos dá uma pista de como fazer isso: a figura 5.6 nos mostra uma comparação entre o número total de vezes que a sub-rotina DFS é chamada (em verde) com o número de vezes em que a chamada dessa sub-rotina visita apenas um elemento. Por exemplo, para instâncias de tamanho 16, cerca de $40 \%$ das chamadas de DFS visitam um único elemento, isto é, uma busca de início de caminho é chamada para remover do espaço de busca corrente um único elemento.

Portanto, uma possível abordagem a ser estudada seria a realização de múltiplas buscas em profundidade: ou seja, ao chamar a sub-rotina DFS, a mesma não executaria até o fim da execução, 


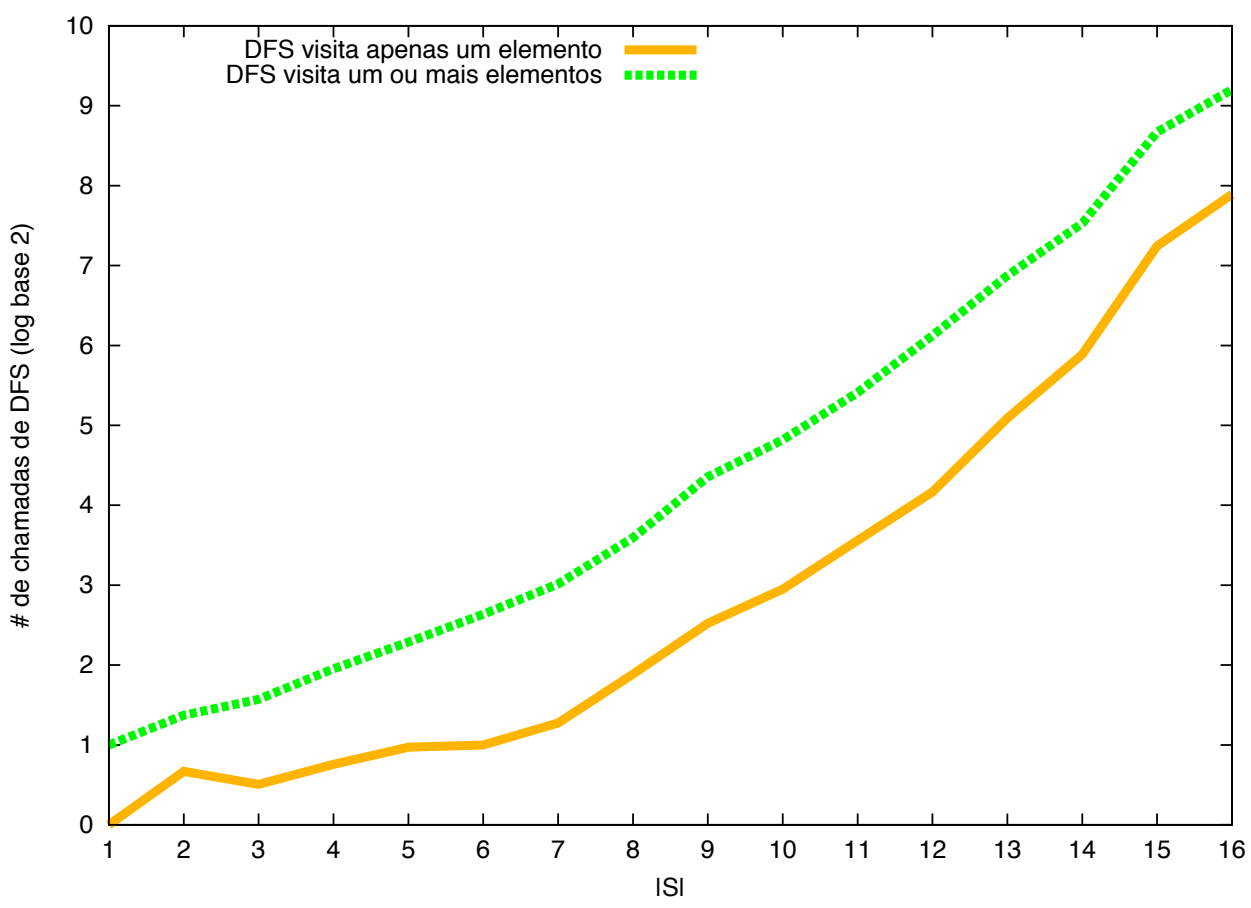

Figura 5.6: comparação entre o número total de vezes que a sub-rotina DFS é chamada (linha tracejada) com o número de vezes em que a chamada dessa sub-rotina visita apenas um elemento (linha sólida), durante a execução do algoritmo UCS-3 no experimento mostrado na tabela 5.3. Observe que para instâncias de tamanho 16 cerca de metade das chamadas dessa sub-rotina visitam um único elemento.

mas sim abortaria a mesma dentro de algum critério (e.g., tamanho do subgrafo), devolvendo o controle ao algoritmo principal juntamente com o subgrafo no estado em que a busca foi interrompida. A ideia é que um ajuste fino entre o número desses subgrafos e número de buscas de início de caminho levem a um melhor desempenho do algoritmo.

\subsubsection{Operações de interseções de conjuntos}

Existe um resultado teórico de operações de conjuntos que indica uma maneira de resolver uma generalização do problema da busca de início de caminho. Em tal generalização o objetivo não é obter um elemento $X$ do espaço de busca corrente, mas sim um intervalo $[A, B]$ do mesmo.

Problema 5.4.1. (problema da busca de intervalo) Sejam $S$ um conjunto finito e não-vazio, $\mathcal{R}_{L}$ e $\mathcal{R}_{U}$ coleções de restrições, respectivamente, inferiores e superiores, cujos tamanhos são polinomiais em $|S|$. Seja $\mathcal{X}\left(\mathcal{R}_{L}, \mathcal{R}_{U}\right)$ um espaço de busca corrente do espaço de busca $\mathcal{P}(S)$. Encontrar um intervalo $[A, B]$ de $\mathcal{X}\left(\mathcal{R}_{L}, \mathcal{R}_{U}\right)$.

O problema de busca de intervalo (problema 5.4.1) pode ser descrito como a busca de um 
intervalo não-vazio obtido a partir da equação:

$$
\begin{aligned}
\mathcal{X}\left(\mathcal{R}_{L}, \mathcal{R}_{U}\right) & =\mathcal{P}(S)-\bigcup_{R \in \mathcal{R}_{L}}[\emptyset, R]-\bigcup_{R \in \mathcal{R}_{U}}[R, S] \\
& =\mathcal{P}(S) \cap\left(\bigcup_{R \in \mathcal{R}_{L}}[\emptyset, R]\right)^{c} \cap\left(\bigcup_{R \in \mathcal{R}_{U}}[R, S]\right)^{c} \\
& =\left(\bigcup_{R \in \mathcal{R}_{L}}[\emptyset, R]\right)^{c} \cap\left(\bigcup_{R \in \mathcal{R}_{U}}[R, S]\right)^{c} \\
& =\left(\bigcap_{R \in \mathcal{R}_{L}}[\emptyset, R]^{c}\right) \cap\left(\bigcap_{R \in \mathcal{R}_{U}}[R, S]^{c}\right) \\
& =\left(\bigcap_{R \in \mathcal{R}_{L}}\left(\bigcup_{r \in R^{c}}[\{r\}, S]\right)\right) \cap\left(\bigcap_{R \in \mathcal{R}_{U}}\left(\bigcup_{r \in R}\left[\emptyset,\{r\}^{c}\right]\right)\right)
\end{aligned}
$$

(proposições 3.2 e 3.3 de Barrera e Salas, 1996 [BS96])

$$
=\left(\bigcap_{R \in \mathcal{R}_{L}}\left(\left[\left\{r_{1}\right\}, S\right] \cup \ldots \cup\left[\left\{r_{\left|R^{c}\right|}\right\}, S\right]\right)\right) \cap\left(\bigcap_{R \in \mathcal{R}_{U}}\left(\left[\emptyset,\left\{r_{1}\right\}^{c}\right] \cup \ldots \cup\left[\emptyset,\left\{r_{|R|}\right\}^{c}\right]\right)\right)
$$

$$
=\left(\left(\left[\left\{r_{11}\right\}, S\right] \cap \ldots \cap\left[\left\{r_{\left|\mathcal{R}_{L}\right| 1}\right\}, S\right]\right) \cup \ldots \cup\left(\left[\left\{r_{1\left|R_{1}^{c}\right|}\right\}, S\right] \cap \ldots \cap\left[\left\{r_{\left|\mathcal{R}_{L}\right|\left|R_{\mid \mathcal{R}_{L}}^{c}\right|}\right\}, S\right]\right)\right) \cap
$$

$\cap\left(\left(\left[\emptyset,\left\{r_{11}\right\}^{c}\right] \cap \ldots \cap\left[\emptyset,\left\{r_{\left|\mathcal{R}_{U}\right| 1}\right\}^{c}\right]\right) \cup \ldots \cup\left(\left[\emptyset,\left\{r_{1\left|R_{1}\right|}\right\}^{c}\right] \cap \ldots \cap\left[\emptyset,\left\{r_{\left|\mathcal{R}_{U} \| R_{\left|\mathcal{R}_{U}\right|}\right|}\right\}^{c}\right]\right)\right)$

(propriedade associativa de intervalos)

$$
\begin{aligned}
= & \left(( [ \{ r _ { 1 1 } \} \cup \ldots \cup \{ r _ { | \mathcal { R } _ { L } | 1 } \} , S ] ) \cup \ldots \cup \left(\left[\{ r _ { 1 | R _ { 1 } ^ { c } | } \} \cup \ldots \cup \left\{r_{\left.\left.\left.\left.\left|\mathcal{R}_{L}\right| \mid R_{\left|\mathcal{R}_{L}\right|}^{c \mid}\right\}, S\right]\right)\right) \cap}\right.\right.\right.\right. \\
& \cap\left(\left(\left[\emptyset,\left\{r_{11}\right\}^{c} \cup \ldots \cup\left\{r_{\left|\mathcal{R}_{U}\right| 1}\right\}^{c}\right]\right) \cup \ldots \cup\left(\left[\emptyset,\left\{r_{1\left|R_{1}\right|}\right\}^{c} \cup \ldots \cup\left\{r_{\left|\mathcal{R}_{U}\right|\left|R_{\left|\mathcal{R}_{U}\right|}\right|}\right\}^{c}\right]\right)\right)
\end{aligned}
$$

(propriedade de interseção de intervalos).

A última igualdade da equação 5.3 nos fornece uma maneira de calcular um intervalo $[A, B] \subseteq$ $\mathcal{X}\left(\mathcal{R}_{L}, \mathcal{R}_{U}\right)$, tal que $A$ seja minimal em $\mathcal{X}\left(\mathcal{R}_{L}, \mathcal{R}_{U}\right)$ e $B$ seja maximal no espaço de busca corrente $\mathcal{X}\left(\mathcal{R}_{L}, \mathcal{R}_{U}\right)$. Para tanto, basta tomar a primeira interseção não-vazia entre um termo $A$ da primeira sequência de uniões e um termo $B$ da segunda sequência de uniões para assim obter um intervalo $[A, B]$. Observe que $A$ e $B$ são inícios de caminho ( $B$ maximal e $A$ minimal). Pela equação 5.3 , a inexistência de um intervalo $[A, B]$ não-vazio implica que o espaço de busca corrente é vazio.

Uma vantagem de se obter um intervalo $[A, B] \subseteq \mathcal{X}\left(\mathcal{R}_{L}, \mathcal{R}_{U}\right)$ ao invés de apenas um início de caminho é que $[A, B]$ delimita uma área que certamente pertence ao espaço de busca; dessa forma, durante a execução da sub-rotina DFS, sempre que a mesma visitar um elemento $X$ que pertença a $[A, B]$, não é necessário verificar as coleções de restrições para saber se $X$ pertence ao espaço de busca corrente; tal economia de consulta às coleções de restrições pode acarretar em um melhor desempenho da sub-rotina.

Todavia, uma limitação da busca de um início de caminho computando-se diretamente as in- 
terseções de intervalos é o número de termos das sequências de uniões, que cresce explosivamente em função dos tamanhos de $\mathcal{R}_{L}$ e de $\mathcal{R}_{U}$ : o número de termos das duas sequências de uniões é $O\left(|S|^{\left|\mathcal{R}_{L}\right|+\left|\mathcal{R}_{U}\right|}\right)$, o que é intratável mesmo para instâncias não muito grandes. Dessa forma, faz-se necessária uma estratégia que minimize o recálculo de interseções já computadas em iterações anteriores. Uma primeira abordagem a ser testada é resolver o problema da busca de intervalo como um problema de Programação Linear Inteira.

\section{Problema 5.4.2.}

$$
\begin{aligned}
\text { maximizar } & \left(|S|-\sum_{i \in S} a_{i}\right)+\sum_{i \in S} b_{i}, \\
\text { sujeito } a & \sum_{i \notin R} a_{i} \geq 1, \forall R \in \mathcal{R}_{L}, \\
& \sum_{i \in R} a_{i} \leq|R|-1, \forall R \in \mathcal{R}_{U}, \\
& \sum_{i \notin R} b_{i} \geq 1, \forall R \in \mathcal{R}_{L}, \\
& \sum_{i \in R} b_{i} \leq|R|-1, \forall R \in \mathcal{R}_{U}, \\
& a_{i} \leq b_{i}, \forall i \in S, \\
& a_{i} \in\{0,1\}, \forall i \in S, \\
& b_{i} \in\{0,1\}, \forall i \in S .
\end{aligned}
$$

Se uma instância do problema 5.4.2 não for viável, então a instância equivalente do problema 5.4.1 não tem solução. Caso contrário, uma solução $\langle\mathbf{a}, \mathbf{b}\rangle$ é equivalente aos vetores característicos das extremidades de um intervalo $[A, B]$ de $\mathcal{X}\left(\mathcal{R}_{L}, \mathcal{R}_{U}\right)$ tal que $|[A, B]|$ seja máximo (i.e., não existe um outro intervalo $[C, D]$ de $\mathcal{X}\left(\mathcal{R}_{L}, \mathcal{R}_{U}\right)$ tal que $\left.|[C, D]|>|[A, B]|\right)$.

\subsubsection{Aninhamento de coleções de restrições e grafo tripartido}

Esta abordagem explora os casos em que as coleções de restrições apresentam uma propriedade adicional.

Definição 5.4.3. Sejam $\mathcal{R}_{L}, \mathcal{R}_{U} \subseteq \mathcal{P}(S)$ coleções de restrições inferiores e superiores, respectivamente. Se valem as seguintes propriedades:

(i) Para cada $L$ em $\mathcal{R}_{L}$, existe um $U$ em $\mathcal{R}_{U}$, tal que $U \subseteq L$;

(ii) Para cada $U$ em $\mathcal{R}_{U}$, existe um $L$ em $\mathcal{R}_{L}$, tal que $U \subseteq L$.

Então diz-se que $\mathcal{R}_{L}$ e $\mathcal{R}_{U}$ são aninhadas.

A sub-rotina DFS quase sempre devolve duas coleções de restrições que são aninhadas. Portanto, para fazermos uso desta propriedade, essa sub-rotina precisaria ser modificada de forma a garantir que as coleções de restrições devolvidas sempre sejam aninhadas.

O aninhamento das coleções de restrições pode ser explorado para acelerar a busca por um elemento mínimo, pois ele é informativo a respeito de alguns elementos que não precisam ser visitados durante uma busca.

Corolário 5.4.4. Sejam $S$ um conjunto e $k$ um inteiro não-negativo. Sejam $\mathcal{R}_{L}$ e $\mathcal{R}_{U}$ coleções de restrições inferiores e superiores, respectivamente, e que são aninhadas. Todos os elementos de $\mathcal{P}(S)$ que contenham os (estejam contidos nos) elementos de $\mathcal{R}_{L}\left(\mathcal{R}_{U}\right)$ não pertencem ao espaço de busca corrente $\mathcal{X}\left(\mathcal{R}_{L}, \mathcal{R}_{U}\right)$. 


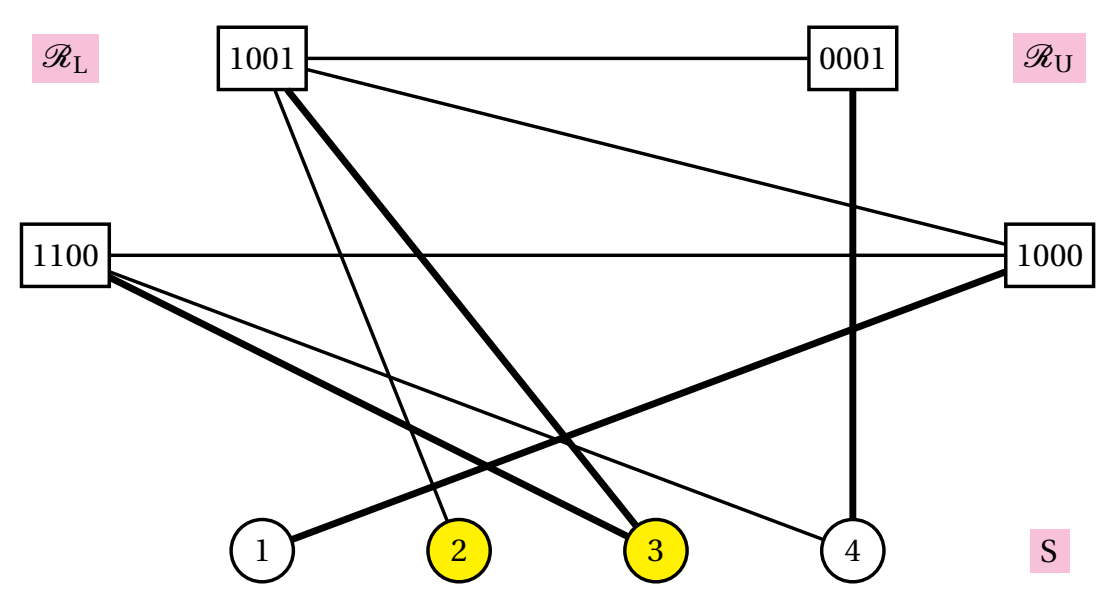

Figura 5.7: um exemplo de grafo tripartido para representar os 4 elementos de um conjunto $S$, duas coleções de restrições (no caso, $\mathcal{R}_{L}=\{1100,1001\}$ e $\mathcal{R}_{U}=\{1000,0001\}$ ) que são aninhadas, as interseções entre os elementos de uma mesma coleção e também entre os elementos de coleções distintas.

Uma das possibilidades de exploração das informações fornecidas pelo alinhamento das coleções de restrições é a utilização de uma nova estrutura de dados para este fim. A figura 5.7 é um exemplo de tal estrutura de dados; no caso, um grafo tripartido.

O aninhamento das coleções de restrições também pode ser explorado adicionando duas restrições ao problema de Programação Linear Inteira que modela o problema da busca de início de caminho.

Problema 5.4.5.

$$
\begin{aligned}
\text { minimizar (maximizar) } & \sum_{i \in S} x_{i}, \\
\text { sujeito a } & \sum_{i \notin R} x_{i} \geq 1, \forall R \in \mathcal{R}_{L}, \\
& \sum_{i \in R} x_{i} \leq|R|-1, \forall R \in \mathcal{R}_{U}, \\
& \sum_{i \in R} x_{i} \leq|R|-1, \forall R \in \mathcal{R}_{L}, \\
& \sum_{i \notin R} x_{i} \geq 1, \forall R \in \mathcal{R}_{U}, \\
& x_{i} \in\{0,1\}, \forall i \in S .
\end{aligned}
$$

\subsection{Comentários}

Seja $\psi$ uma função que leva valores de $\mathcal{P}(S)$ em $\{0,1\}$, chamada de mapeamento de conjunto (set mapping). Um núcleo (kernel) é o subconjunto dos elementos de $\mathcal{P}(S)$ para os quais o valor de $\psi$ é igual a um. Embora um núcleo sirva para representar a função $\psi$, o mesmo é muito grande para ser utilizado diretamente [Hei95]. Este fato foi visto na prática, durante o desenvolvimento do algoritmo U-CuRVE: as primeiras versões do mesmo utilizavam um núcleo como representação do espaço de busca corrente, o que se mostrou inviável mesmo para instâncias pequenas [Bar12].

Um subconjunto $\mathcal{X} \subseteq \mathcal{P}(S)$ é convexo se para todo $A, B \in \mathcal{X}, A \subseteq B$ implicar em $[A, B] \subseteq \mathcal{X}$ (Birkhoff, 1967, página 7 [Bir67]). Subconjuntos convexos definem o núcleo de importantes tipos de mapeamento de conjuntos, como por exemplo, o mapeamento inf-separável invariante por translação (inf-separable t.i. mapping) [BB91].

Um espaço de busca corrente $\mathcal{X}\left(\mathcal{R}_{L}, \mathcal{R}_{U}\right)$, definido através da coleção $\mathcal{R}_{L}$ de restrições inferiores e da coleção $\mathcal{R}_{U}$ de restrições superiores, é um subconjunto convexo. Dessa forma, o mesmo pode 
ser utilizado, no contexto de Morfologia Matemática, como uma forma de representação do núcleo de algumas classes de mapeamento de conjuntos (i.e., daquelas que são isomorfas a subconjuntos convexos). Portanto, o problema da busca de início de caminho (problema 5.1.1) pode ser visto, dentro deste novo contexto, como o problema de se obter um elemento do núcleo de um mapeamento de conjunto representado de forma indireta, através de coleções de restrições. 


\section{Capítulo 6}

\section{Algoritmos branch-and-bound para o problema U-curve}

Apresentaremos neste capítulo dois algoritmos branch-and-bound para abordar o problema Ucurve. A motivação inicial para o desenvolvimento desta família de algoritmos foi proporcionar uma melhor avaliação do algoritmo UCS (apresentado no capítulo 4) do que a possibilitada pela comparação com a busca exaustiva: o algoritmo branch-and-bound descrito por Narendra e Fukunaga, que foi apresentado no capítulo 2, não é um benchmark adequado para ser utilizado na comparação, haja vista que o mesmo trabalha com a hipótese da função custo ser monótona (o que não é o caso de todas as funções custo decomponíveis em curvas em U) e com a fixação de uma cardinalidade arbitrária para o conjunto de custo mínimo a ser devolvido (portanto, este algoritmo resolve um problema diferente do problema U-curve) [NF77]. Dessa forma, desenvolvemos o algoritmo U-CURVE-BRANCH-AND-Bound (UBB), um branch and bound ótimo para abordar o problema U-curve, que implementa os princípios de funcionamento esboçados em Ris e colegas, figura 8 [RBMJ10]. A partir do algoritmo UBB, desenvolvemos o algoritmo Poset-Forest-SEARCH (PFS), que generaliza a dinâmica de UBB e que também se trata de um algoritmo ótimo para abordar o problema U-curve.

Iniciaremos este capítulo apresentando o algoritmo UBB, assim como uma simulação de execução, pseudo-código do algoritmo e de sua sub-rotina, prova de corretude e análise de complexidade de tempo. Em seguida, mostraremos as limitações de UBB que levaram ao desenvolvimento de uma generalização deste algoritmo. Introduziremos essa generalização, o algoritmo PFS, assim como seus princípios de funcionamento, simulação de execução, pseudo-código e análise de complexidade de tempo. Na sequência, serão mostrados alguns experimentos feitos com os dois algoritmos, assim como algumas análises sobre os resultados obtidos. Por fim, concluiremos fazendo alguns comentários sobre possíveis trabalhos futuros com os algoritmos apresentados neste capítulo.

\subsection{O algoritmo U-CURVE-BRANCH-AND-BOUnd (UBB)}

U-CURVE-BRANCH-AND-BOUnd (UBB) é um algoritmo branch-and-bound ótimo desenvolvido para abordar o problema U-curve. A ideia geral deste algoritmo é utilizar uma árvore $T$ como espaço de busca. $T$ é um sub-grafo do diagrama de Hasse do reticulado Booleano $(\mathcal{P}(S), \subseteq)$ e é construído implicitamente através de um esquema de enumeração; tal esquema utiliza aplicações recursivas da seguinte decomposição do espaço de busca.

Lema 6.1.1. Sejam $X$ e $Y$ conjuntos, $X$ não-vazio. Seja $X_{0} \supseteq X_{1} \supseteq \ldots \supseteq X_{|X|}$ uma cadeia tal que $X_{0}=X, X_{|X|}=\emptyset$ e $X_{i} \cup\left\{x_{i}\right\}=X_{i-1}$ para todo $0<i \leq|X|$. Vale que:

$$
\{Y\} \cup \bigcup_{i=1}^{|X|}\left\{W \cup Y \cup\left\{x_{i}\right\}: W \in \mathcal{P}\left(X_{i}\right)\right\}=\{W \cup Y: W \in \mathcal{P}(X)\} .
$$


Demonstração. Esta prova é uma indução em $|X|$. Se $|X|=0$, então vale que:

$$
\begin{aligned}
\{Y\} \cup \bigcup_{i=1}^{0}\left\{W \cup Y \cup\left\{x_{i}\right\}: W \in \mathcal{P}\left(X_{i}\right)\right\} & =\{Y\} \cup \emptyset \\
& =\{W \cup Y: W \in \emptyset\} \\
& =\{W \cup Y: W \in \mathcal{P}(\emptyset)\} \\
& =\{W \cup Y: W \in \mathcal{P}(X)\} .
\end{aligned}
$$

Agora, consideremos quando $|X|>0$. Vale que:

$$
\begin{aligned}
& \{Y\} \cup \bigcup_{i=1}^{|X|}\left\{W \cup Y \cup\left\{x_{i}\right\}: W \in \mathcal{P}\left(X_{i}\right)\right\}= \\
& =\{Y\} \cup\left\{W \cup Y \cup\left\{x_{1}\right\}: W \in \mathcal{P}\left(X_{1}\right)\right\} \cup \bigcup_{i=2}^{|X|}\left\{W \cup Y \cup\left\{x_{i}\right\}: W \in \mathcal{P}\left(X_{i}\right)\right\} \\
& =\{Y\} \cup\left\{W \cup Y \cup\left\{x_{1}\right\}: W \in \mathcal{P}\left(X_{1}\right)\right\} \cup \bigcup_{j=1}^{|Z|}\left\{W \cup Y \cup\left\{z_{j}\right\}: W \in \mathcal{P}\left(Z_{j}\right)\right\} \\
& \quad\left(Z=Z_{0}=X_{1}, \ldots, Z_{|Z|}=X_{|X|}\right) \\
& =\left\{W \cup Y \cup\left\{x_{1}\right\}: W \in \mathcal{P}\left(X_{1}\right)\right\} \cup\{W \cup Y: W \in \mathcal{P}(Z)\} \\
& \quad(\text { pela hipótese de indução) } \\
& =\left\{W \cup Y \cup\left\{x_{1}\right\}: W \in \mathcal{P}\left(X_{1}\right)\right\} \cup\left\{W \cup Y: W \in \mathcal{P}\left(X_{1}\right)\right\} \\
& \quad\left(\text { uma vez que } X_{1}=Z\right) \\
& =\left\{W \cup Y: W \in \mathcal{P}\left(X_{1} \cup\left\{x_{1}\right\}\right)\right\} \\
& \left.\quad \text { (uma vez que } \mathcal{P}\left(X_{1} \cup\left\{x_{1}\right\}\right)=\mathcal{P}\left(X_{1}\right) \cup\left\{W \cup\left\{x_{1}\right\}: W \in \mathcal{P}\left(X_{1}\right)\right\}\right) \\
& =\{W \cup Y: W \in \mathcal{P}(X)\} \\
& \quad\left(\text { uma vez que } X=X_{1} \cup\left\{x_{1}\right\}\right) .
\end{aligned}
$$

Durante uma busca, a árvore $T$ é podada utilizando o fato de que a função custo $c$ é decomponível em curvas em U: o algoritmo ramifica (branches) até que o custo de um elemento visitado comece a subir, atingindo assim seu limite (bound); caso isto ocorra, então a árvore é podada (pruned).

Nas figuras 6.1-6.4 mostramos uma simulação de UBB. Este algoritmo é simulado da seguinte maneira: 


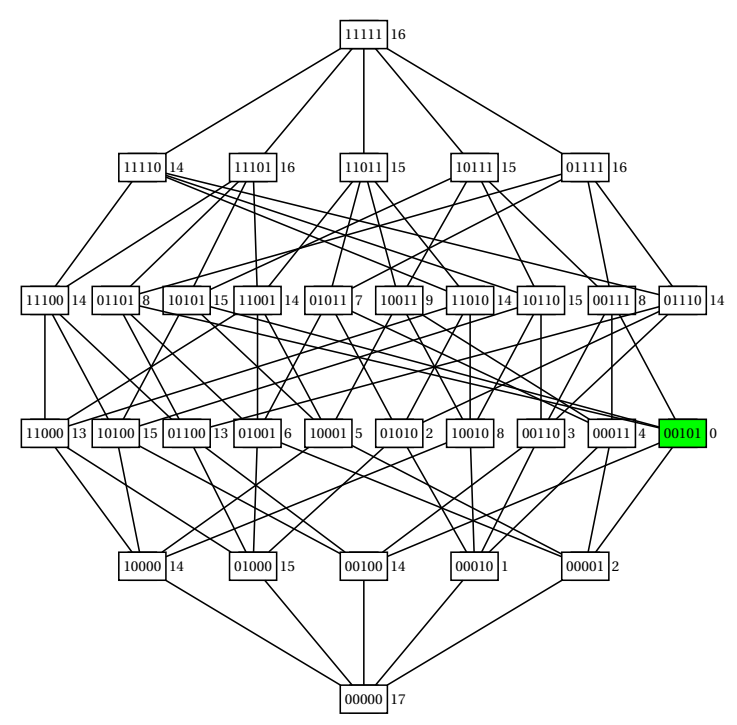

(a)

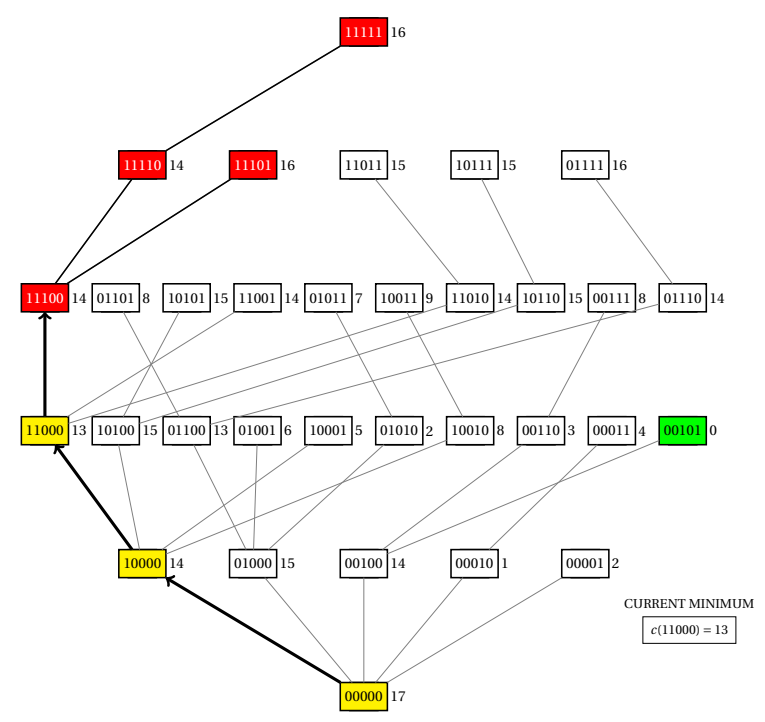

(c)

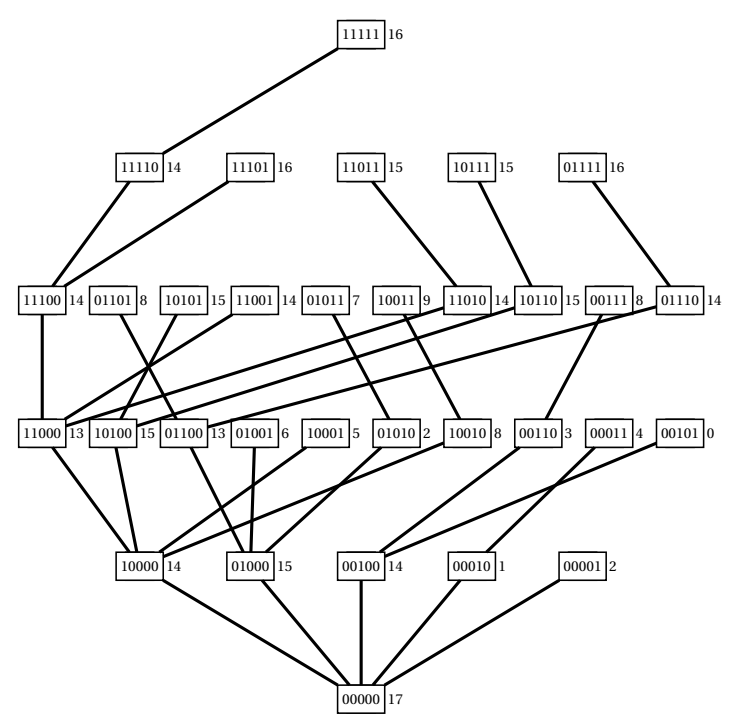

(b)

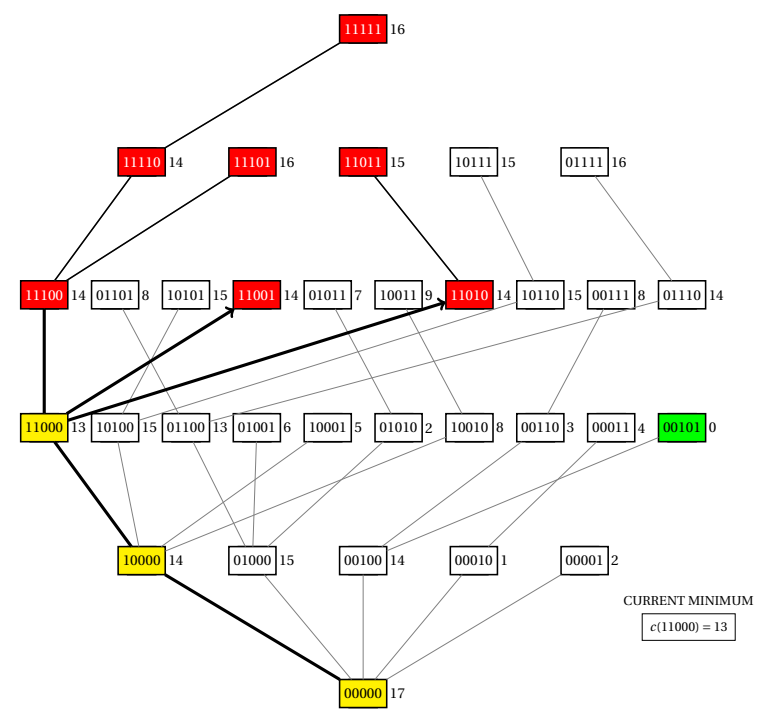

(d)

Figura 6.1: uma simulação de U-CURVE-BRANCH-AND-BOUND, um algoritmo branch-and-bound para abordar o problema U-curve.

- Figura 6.1(a): uma instância com cinco elementos e função custo $c$, esta última definida pelos números ao lado dos nós; o elemento 00101 (em verde) tem custo mínimo;

- Figura 6.1(b): uma enumeração arbitrária para esta instância, que utiliza a decomposição mostrada no lema 6.1.1: dados elementos $X$ e $Y$ do reticulado Booleano tal que $Y$ é adjacente superior a $X, Y$ é visitado a partir de $X$ se e somente se $Y$ é $X$ mais um bit à direita do bit mais à direita de $X$. Por exemplo, se $X=00100$, então 00101 é visitado a partir de $X$ e 01100 não é;

- Figura 6.1(c): o algoritmo ramifica até o elemento 11000; uma vez que $c(11100)>c(11000)$, a sub-árvore que tem 11100 como raiz é podada do espaço de busca;

- Figura 6.1(d): 11010 e 11001 são verificados; uma vez que ambos têm custo maior que o de 11000, suas respectivas sub-árvores são podadas do espaço de busca; 


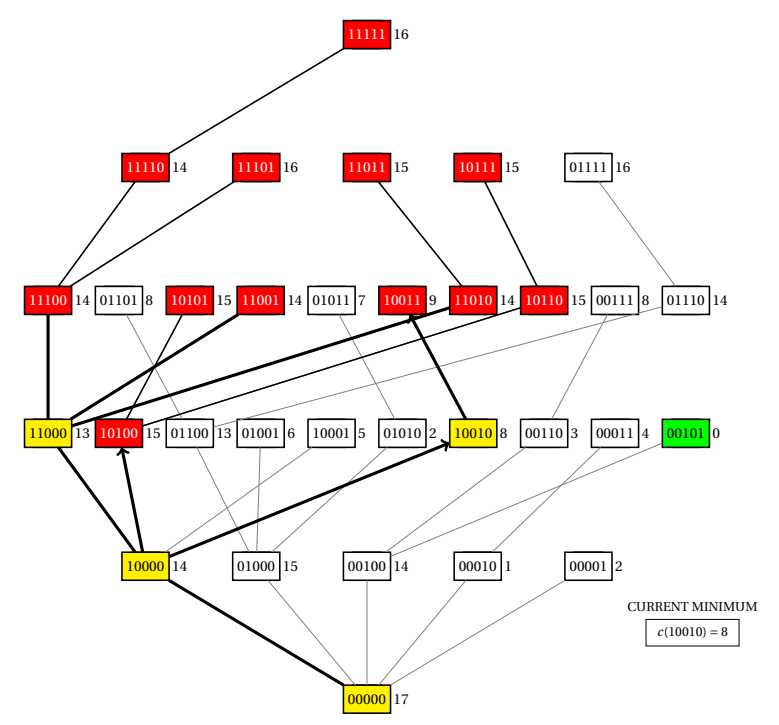

(a)

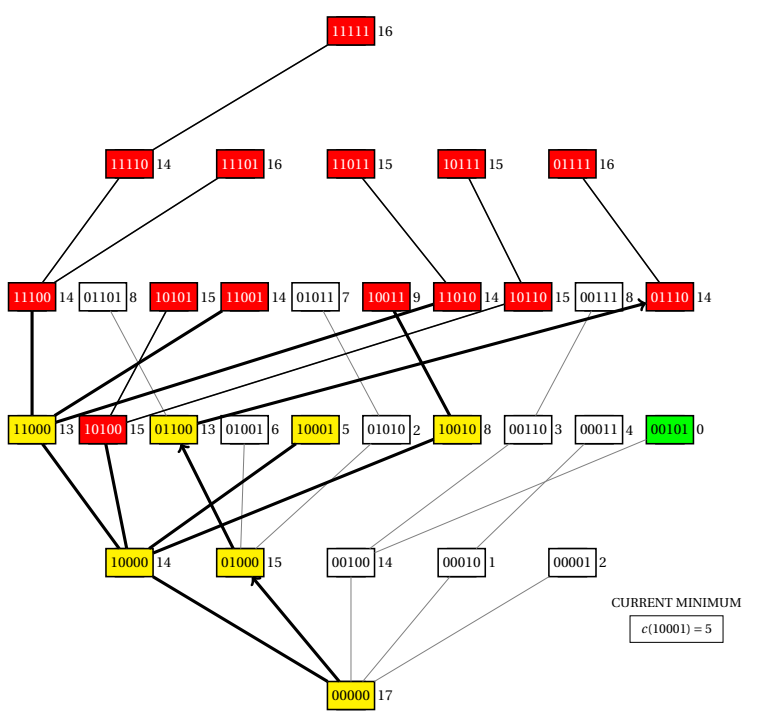

(c)

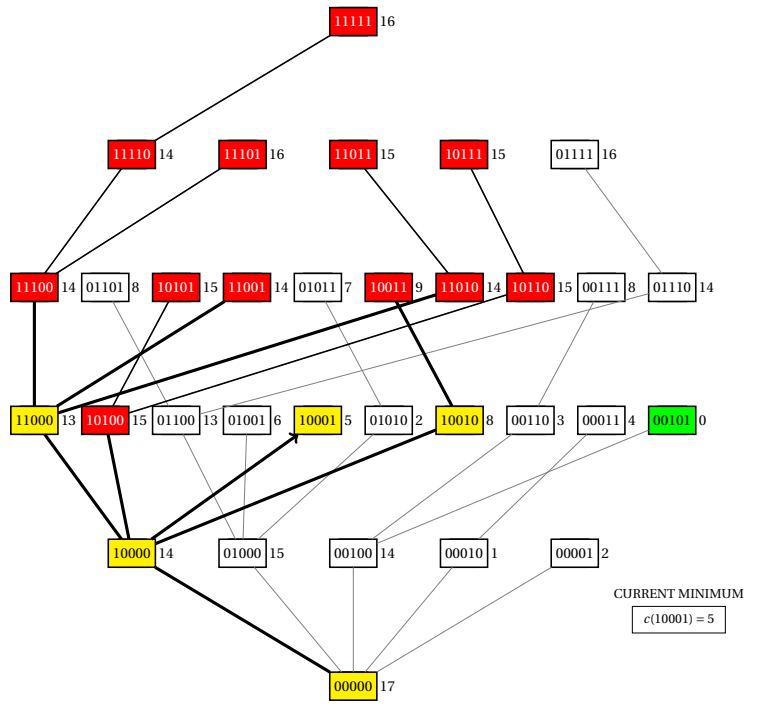

(b)

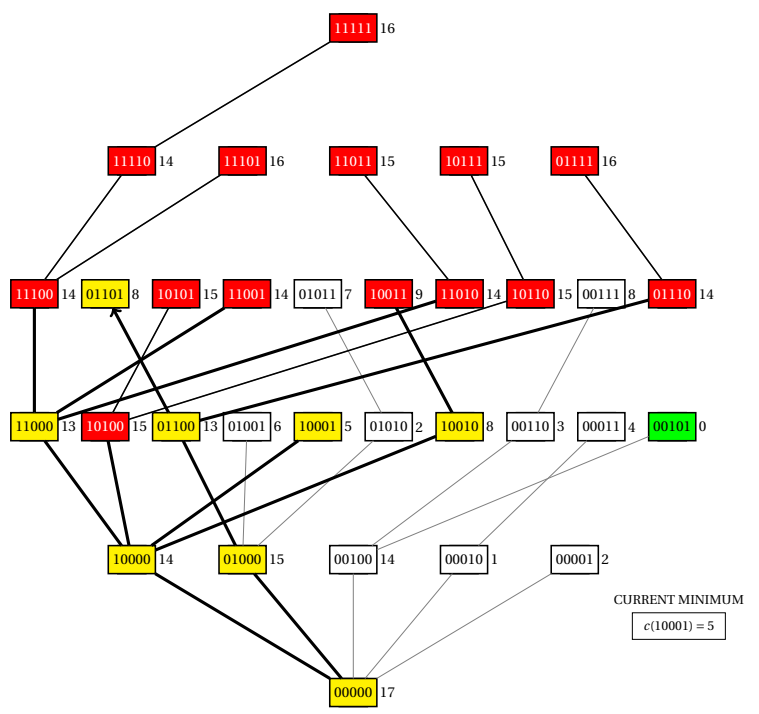

(d)

Figura 6.2: continuação da simulação da figura 6.1.

- Figura 6.2(a): 11000 não tem mais possibilidades de ramificação, portanto o algoritmo recua (backtracks). O algoritmo então ramifica até o elemento 10100. Uma vez que $c(10100)>$ $c(10000)$, a sub-árvore que tem 10100 como raiz é podada do espaço de busca. O algoritmo ramifica até o elemento 10010; uma vez que $c(10011)>c(10010)$, a sub-árvore que tem 10011 como raiz é podada do espaço de busca;

- Figura 6.2(b): 10010 não tem mais possibilidades de ramificação, portanto o algoritmo recua. O algoritmo ramifica até o elemento 10001;

- Figura 6.2(c): 10001 e 10000 não têm mais possibilidades de ramificação, portanto o algoritmo recua duas vezes. O algoritmo ramifica até o elemento 01100; uma vez que $c(01110)>$ $c(01100)$, a sub-árvore que tem 01110 como raiz é podada do espaço de busca;

- Figura 6.2(d): o algoritmo ramifica até o elemento 01101; 


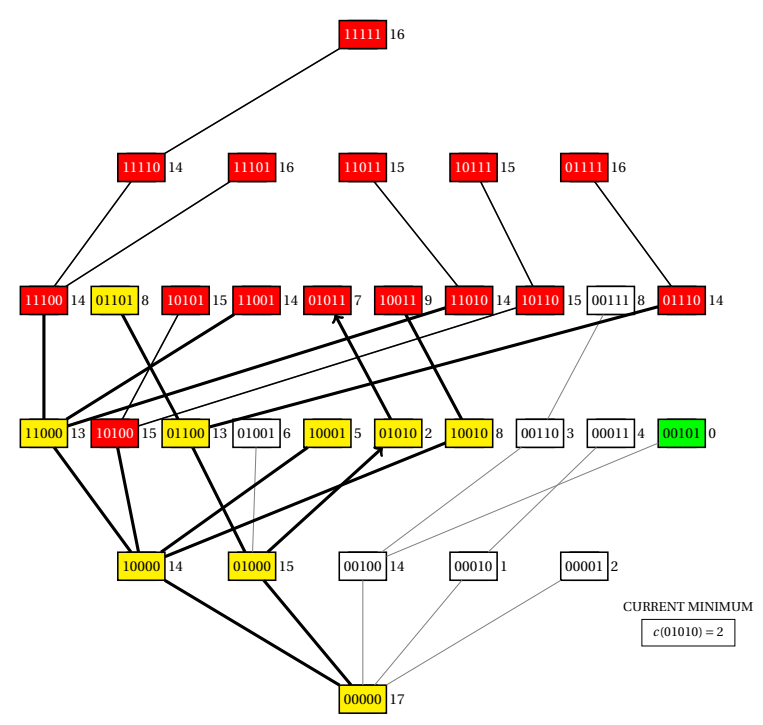

(a)

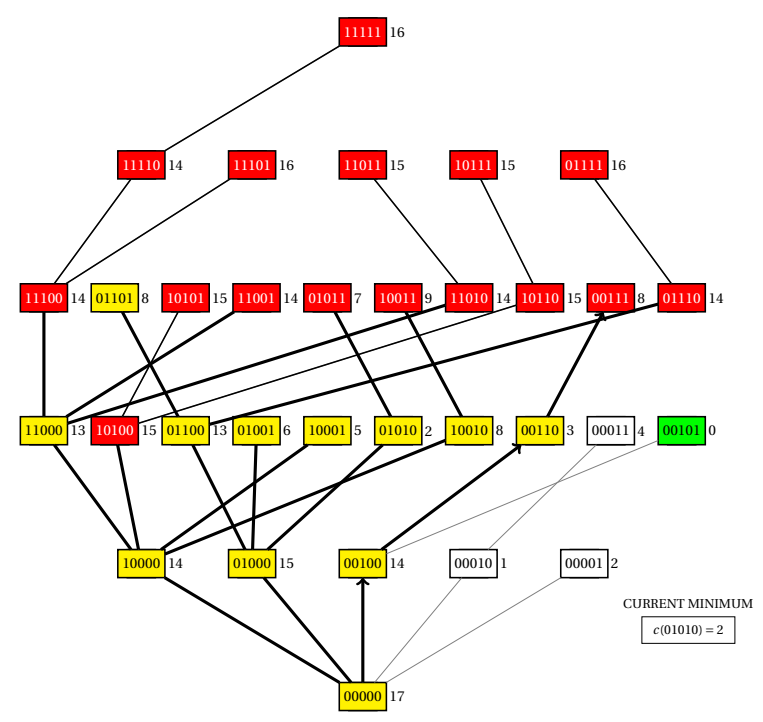

(c)

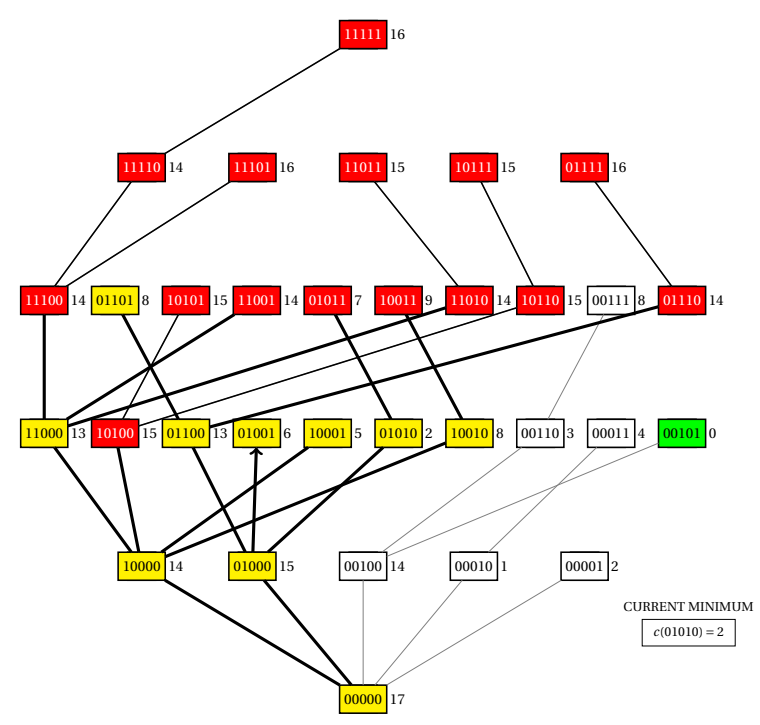

(b)

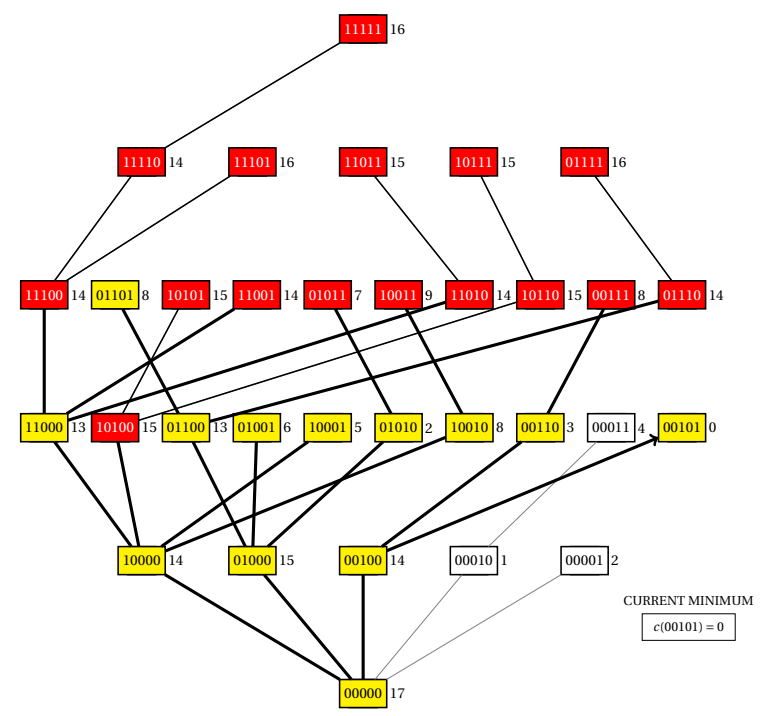

(d)

Figura 6.3: continuação da simulação da figura 6.2.

- Figura 6.3(a): 01101 e 01100 não têm mais possibilidades de ramificação, portanto o algoritmo recua duas vezes. O algoritmo ramifica até o elemento 01010; uma vez que $c(01011)>$ $c(01010)$, a sub-árvore que tem 01011 como raiz é podada do espaço de busca;

- Figura 6.3(b): 01010 não tem mais possibilidades de ramificação, portanto o algoritmo recua. O algoritmo ramifica até o elemento 01001;

- Figura 6.3(c): 01001 e 01000 não têm mais possibilidades de ramificação, portanto o algoritmo recua duas vezes. O algoritmo ramifica até o elemento 00110; uma vez que $c(00111)>$ $c(00110)$, a sub-árvore que tem 00111 como raiz é podada do espaço de busca;

- Figura 6.3(d): 00110 não tem mais possibilidades de ramificação, portanto o algoritmo recua. O algoritmo ramifica até o elemento 00101; 


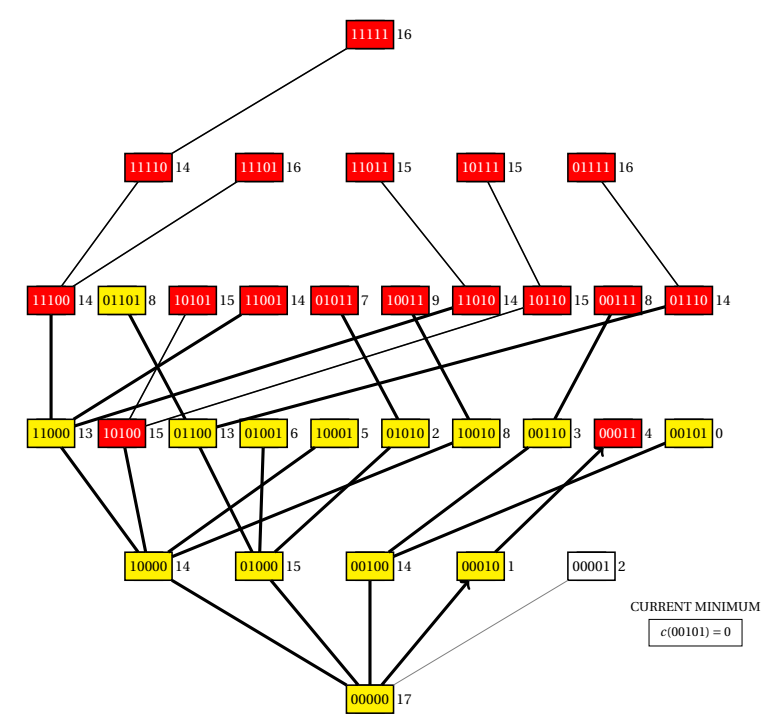

(a)

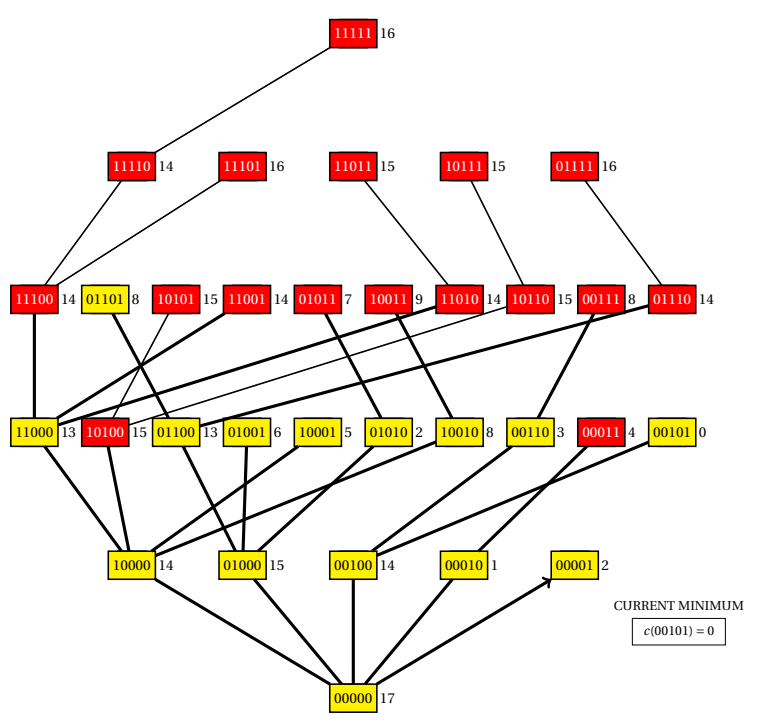

(b)

Figura 6.4: continuação da simulação da figura 6.3.

- Figura 6.4(a): 00101 e 00100 não têm mais possibilidades de ramificação, portanto o algoritmo recua duas vezes. O algoritmo ramifica até o elemento 00010; uma vez que $c(00011)>$ $c(00010)$, a sub-árvore que tem 00011 como raiz é podada do espaço de busca;

- Figura 6.4(b): 00010 não tem mais possibilidades de ramificação, portanto o algoritmo recua. O algoritmo ramifica até o elemento 00001; 00001 não tem mais possibilidades de ramificação, portanto o algoritmo recua. A raiz da árvore, 00000, não tem mais possibilidades de ramificação, portanto a execução do algoritmo é encerrada.

Apresentaremos agora o pseudo-código do algoritmo U-CuRVE-BRANCH-AND-Bound, que está dividido em uma sub-rotina e programa principal; para ambos, apresentaremos descrição, prova de corretude e análise de complexidade de tempo.

Descrição de algoritmo. BRANCH recebe um par de conjuntos $X$ e $Y$, uma função custo $c$ decomponível em curvas em $\mathrm{U}$, o custo $\operatorname{cost}_{Y}$ de $Y$, e devolve uma coleção $\mathcal{M}$ que contém todos os elementos de custo mínimo em $\{W \cup Y: W \in \mathcal{P}(X)\}$.

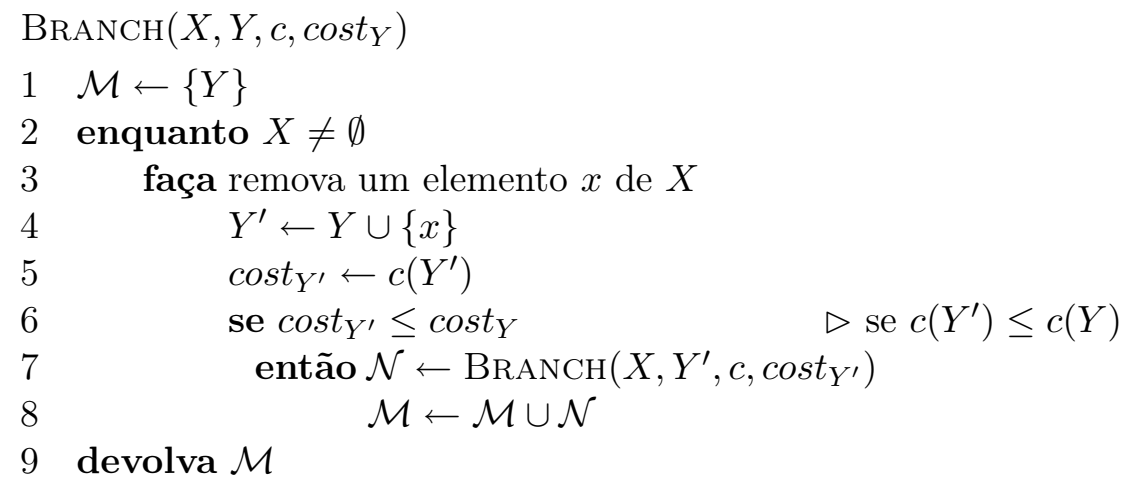

Durante a simulação apresentada a partir da figura 6.1, os passos das figuras 6.1(c)-6.4(b) são equivalentes a uma execução de BrANCH com $X=11111, Y=00000, c$ definida pelos números ao lado dos nós, cost $_{Y}=17$, e a sequência de remoção de elementos de $X$ sempre sendo feita a partir do bit 1 mais à esquerda de $X$. 
Corretude. A seguinte proposição mostra a corretude desta sub-rotina.

Proposição 6.1.2. Se a sub-rotina BRAnch recebe um par de conjuntos $X$ e $Y$, uma função custo c decomponivel em curvas em $U$, e o custo cost $t_{Y}$ de $Y$, então ela devolve uma coleção $\mathcal{M}$ que contém todos os elementos de custo minimo em $\{W \cup Y: W \in \mathcal{P}(X)\}$.

Demonstração. Esta prova é uma indução em $|X|$. Se $|X|=0$, então $X=\emptyset$, logo o laço nas linhas 2-8 não itera, o que implica que é devolvida a coleção $\mathcal{M}=\{Y\}$, que se trata de uma coleção que contém todos os elementos de custo mínimo em:

$$
\begin{aligned}
\{Y\} & =\{W \cup Y: W \in \emptyset\} \\
& =\{W \cup Y: W \in \mathcal{P}(\emptyset)\} \\
& =\{W \cup Y: W \in \mathcal{P}(X)\} .
\end{aligned}
$$

Agora, considere quando $|X|>0$ : o laço nas linhas 2-8 itera $|X|$ vezes. Dada uma iteração $i$, $1 \leq i \leq|X|$, seja $x_{i}$ o elemento a ser removido na linha 3 e seja $X_{i}$ o estado da variável $X$ após a remoção de $x_{i}$. A comparação feita na linha 6 leva a um dos dois casos a seguir:

- $c\left(Y \cup\left\{x_{i}\right\}\right)>c(Y)$ : uma vez que $Y \subseteq Y \cup\left\{x_{i}\right\}$, qualquer elemento em $\left\{W \cup Y \cup\left\{x_{i}\right\}\right.$ : $\left.W \in \mathcal{P}\left(X_{i}\right)\right\}$ contém $Y$. Além disso, qualquer elemento $Z$ em $\left\{W \cup Y \cup\left\{x_{i}\right\}: W \in \mathcal{P}\left(X_{i}\right)\right\}$ tem custo maior que $Y$, pois se $Z$ tivesse um custo menor ou igual a $c(Y)$, então teríamos que $c\left(Y \cup\left\{x_{i}\right\}\right)>\max \{c(Y), c(Z)\}$, o que contradiz a definição de $c$. Dessa forma, a coleção $\left\{W \cup Y \cup\left\{x_{i}\right\}: W \in \mathcal{P}\left(X_{i}\right)\right\}$ é podada do espaço de buscas;

- $c\left(Y \cup\left\{x_{i}\right\}\right) \leq c(Y)$ : neste caso, pela hipótese de indução, a chamada recursiva na linha 7 devolve uma coleção $\mathcal{N}$ que contém todos os elementos de custo mínimo em $\left\{W \cup Y \cup\left\{x_{i}\right\}\right.$ : $\left.W \in \mathcal{P}\left(X_{i}\right)\right\}$.

Portanto, para cada iteração $i$ do laço, uma coleção $\left\{W \cup Y \cup\left\{x_{i}\right\}: W \in \mathcal{P}\left(X_{i}\right)\right\}$ é explorada ou podada. Logo, ao final da última iteração, a coleção de elementos que foram explorados ou podados pelo algoritmo é:

$$
\{Y\} \cup \bigcup_{i=1}^{|X|}\left\{W \cup Y \cup\left\{x_{i}\right\}: W \in \mathcal{P}\left(X_{i}\right)\right\}=\{W \cup Y: W \in \mathcal{P}(X)\} \quad \text { (lema 6.1.1). }
$$

A operação na linha 8 é utilizada para atualizar a coleção $\mathcal{M}$ com todos os elementos de custo mínimo explorados até então. Dessa forma, ao final da última iteração, o algoritmo devolve uma coleção $\mathcal{M}$ que contém todos os elementos de custo mínimo em $\{W \cup Y: W \in \mathcal{P}(X)\}$.

Análise de complexidade de tempo. Seja $b$ o número de vezes que a função custo $c$ é computada. Cada iteração do laço nas linhas 2-8, excluindo a chamada recursiva do algoritmo, consome $O(f(n)+n)$ unidades de tempo. Uma vez que uma chamada recursiva ocorre somente se a comparação na linha 6 é verdadeira, e que $c$ sempre é computada antes dessas comparações, BRANCH consome $O((f(n)+n) b)$ unidades de tempo.

Apresentaremos agora o algoritmo principal.

Descrição de algoritmo. U-CuRVE-BRANCH-AND-Bound recebe um conjunto não-vazio $S$, uma função custo $c$ decomponível em curvas em $\mathrm{U}$, e devolve uma coleção $\mathcal{M} \subseteq \mathcal{P}(S)$ que contém todos os elementos em $\mathcal{P}(S)$ de custo mínimo.

\section{U-CuRVE-Branch-And-Bound $(S, c)$}

$1 \mathcal{M} \leftarrow \operatorname{Branch}(S, \emptyset, c, c(\emptyset))$

2 devolva $\{M \in \mathcal{M}: c(M)$ é mínimo $\}$ 
Durante a simulação mostrada a partir da figura 6.1, os passos das figuras 6.1(c)-6.4(b) são equivalentes a uma execução de UBB com $S=11111$ e $c$ definida pelos números ao lado dos nós.

Corretude. A seguinte proposição mostra a corretude do algoritmo principal.

Proposição 6.1.3. Se UBB recebe uma instância $\langle S, c\rangle$ do problema U-curve, então ele devolve uma coleção $\mathcal{M}$ que contém todos os elementos em $\mathcal{P}(S)$ de custo mínimo.

Demonstração. Se UBB recebe uma instância $\langle S, c\rangle$ do problema U-curve, então na linha 1 desse algoritmo é chamada a sub-rotina BRANCH com parâmetros $X:=S, Y:=\emptyset, Y:=\emptyset$, e cost $t_{Y}:=c(\emptyset)$. Portanto, de acordo com a proposição 6.1.2, BRANCH devolve uma coleção $\mathcal{M}$ que contém todos os elementos de custo mínimo em:

$$
\begin{aligned}
\{W \cup Y: W \in \mathcal{P}(X)\} & =\{W \cup \emptyset: W \in \mathcal{P}(S)\} \\
& =\{W: W \in \mathcal{P}(S)\} \\
& =\mathcal{P}(S) .
\end{aligned}
$$

Uma vez que UBB devolve na linha 2 todos os mínimos dessa coleção $\mathcal{M}$, logo ele devolve uma coleção que contém todos os elementos em $\mathcal{P}(S)$ de custo mínimo.

Análise de complexidade de tempo. A única chamada de BRANCH na linha 1 demanda $O((f(n)+n) b)$ unidades de tempo. Uma vez que uma execução deste algoritmo pode explorar uma fração do espaço de busca que é proporcional ao tamanho total do reticulado Booleano de grau $n$, o limite superior real para o tempo computacional assintótico de UBB é $O\left((f(n)+n) 2^{n}\right)$.

\subsubsection{Limitações do algoritmo UBB}

Como vimos nesta seção, o algoritmo UBB define uma árvore $T$, um sub-grafo do diagrama de Hasse do reticulado Booleano $(\mathcal{P}(S), \subseteq)$, como espaço de busca. $T$ tem como raiz o menor elemento de $(\mathcal{P}(S), \subseteq)$, isto é, o conjunto vazio. O algoritmo percorre $T$ a partir de ramificações iniciadas na raiz da árvore, o que implica que em toda poda são eliminados do espaço de busca os elementos de um intervalo $[Y, X \cup Y]$, no qual $Y$ é o elemento do intervalo que é visitado pelo algoritmo e $[Y, X \cup Y]-\{Y\}$ são os elementos que, pelo esquema de enumeração, poderiam ser visitados a partir de $Y$. Além disso, pela correção da sub-rotina BRANCH, um intervalo $[Y, X \cup Y]$ é eliminado do espaço de busca se e somente se $Y$ é adjacente superior a um elemento de custo menor que o de $Y$. Por exemplo, na figura 6.1(c), o elemento 11100 é adjacente superior ao elemento 11000; como $c(11100)>c(11000)$, o intervalo $[11100,11100 \cup 00011]=[11100,11111]$ é eliminado do espaço de busca.

A estratégia de percorrimento e de poda de UBB apresenta a desvantagem de tornar o algoritmo ineficiente para instâncias cujos elementos visitados que atendam ao critério de poda localizem-se próximos às folhas de $T$. Na figura 6.5 mostramos um exemplo de instância que exige que UBB visite todos os elementos do espaço de busca.

Para tratar de situações tais como a mostrada na figura 6.5, apresentaremos a seguir um novo algoritmo, que generaliza a estratégia de busca de U-CuRVE-BRANCH-AND-BOUnD.

\subsection{O algoritmo Poset-Forest-SeArch (PFS)}

Como vimos na seção anterior, o algoritmo UBB possui limitações decorrentes do fato do mesmo ser unidirecional, ou seja, que o percorrimento inicia-se sempre a partir do menor elemento do reticulado Booleano e que as podas sempre eliminam do espaço de busca um intervalo $[Y, X \cup Y]$, no qual $Y$ é o elemento do intervalo que é visitado pelo algoritmo e $[Y, X \cup Y]-\{Y\}$ são os elementos que, pelo esquema de enumeração, poderiam ser visitados a partir de $Y$. Apresentaremos nesta seção um outro algoritmo branch-and-bound ótimo para o problema U-curve, que generaliza 


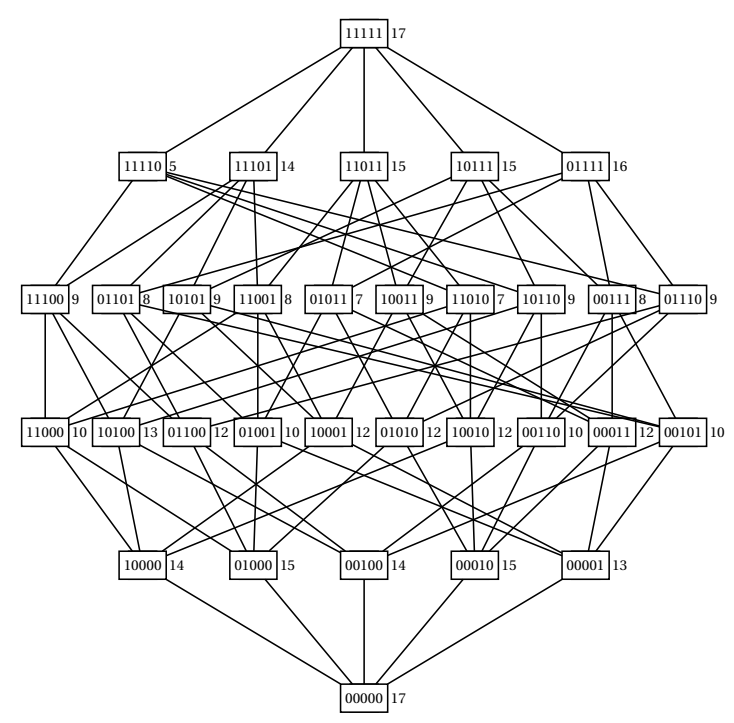

(a)

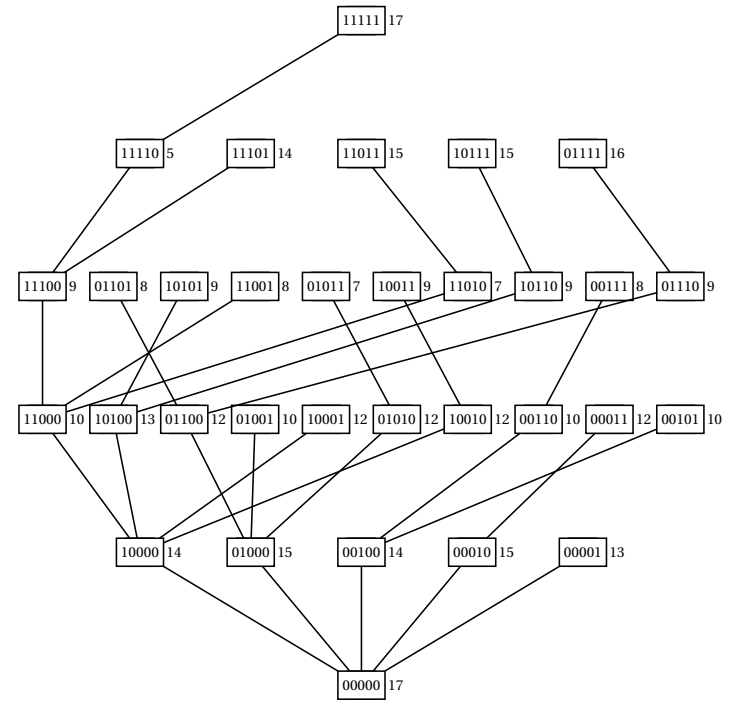

(b)

Figura 6.5: um exemplo de instância que exige que o algoritmo UBB visite todos os elementos do espaço de busca. Figura 6.5(a): o reticulado Booleano definido pelos elementos da instância; Figura 6.5(b): uma enumeração do reticulado Booleano que define uma árvore T; observe que todo elemento de custo minimo nas cadeias definidas por caminhos em $T$ são folhas ou adjacentes inferiores a folhas, o que obriga o algoritmo a visitar todos os vértices de $T$.

a estratégia de busca do algoritmo U-CURVE-BRANCH-AND-Bound; tal generalização consiste em tornar o algoritmo bidirecional, ou seja, que o percorrimento também possa iniciar-se a partir do maior elemento do reticulado Booleano e que as podas também possam eliminar do espaço de busca um intervalo $[Y-X, Y]$, no qual $Y$ é o elemento do intervalo que é visitado pelo algoritmo e $[Y-X, Y]-\{Y\}$ são os elementos que, pelo esquema de enumeração, poderiam ser visitados a partir de $Y$.

\subsubsection{Princípios de funcionamento do novo algoritmo}

Para implementar a ideia de um percorrimento bidirecional, o algoritmo utiliza-se de dois esquemas de enumeração: o primeiro trata-se da decomposição apresentada no lema 6.1.1; já o segundo é a versão dual desse lema.

Lema 6.2.1. Sejam $X$ e $Y$ conjuntos, $X$ não-vazio. Seja $X_{0} \supseteq X_{1} \supseteq \ldots \supseteq X_{|X|}$ uma cadeia tal que $X_{0}=X, X_{|X|}=\emptyset$ e $X_{i} \cup\left\{x_{i}\right\}=X_{i-1}$ para todo $0<i \leq|X|$. Vale que:

$$
\{Y \cup X\} \cup \bigcup_{i=1}^{|X|}\left\{\left(X-\left(W \cup\left\{x_{i}\right\}\right)\right) \cup Y: W \in \mathcal{P}\left(X_{i}\right)\right\}=\{W \cup Y: W \in \mathcal{P}(X)\} .
$$

Demonstração. Esta prova é uma indução em $|X|$. Se $|X|=0$, então vale que:

$$
\begin{aligned}
\{Y \cup X\} \cup \bigcup_{i=1}^{0}\left\{\left(X-\left(W \cup\left\{x_{i}\right\}\right)\right) \cup Y: W \in \mathcal{P}\left(X_{i}\right)\right\} & =\{Y \cup X\} \cup \emptyset \\
& =\{Y \cup \emptyset\} \quad \quad \text { (pois }|X|=0) \\
& =\{W \cup Y: W \in \emptyset\} \quad \\
& =\{W \cup Y: W \in \mathcal{P}(\emptyset)\} \\
& =\{W \cup Y: W \in \mathcal{P}(X)\} .
\end{aligned}
$$


Agora, consideremos quando $|X|>0$. Vale que:

$$
\begin{aligned}
& \{Y \cup X\} \cup \bigcup_{i=1}^{|X|}\left\{\left(X-\left(W \cup\left\{x_{i}\right\}\right)\right) \cup Y: W \in \mathcal{P}\left(X_{i}\right)\right\}= \\
& =\{Y \cup X\} \cup \bigcup_{i=2}^{|X|}\left\{\left(X-\left(W \cup\left\{x_{i}\right\}\right)\right) \cup Y: W \in \mathcal{P}\left(X_{i}\right)\right\} \cup\left\{\left(X-\left(W \cup\left\{x_{1}\right\}\right)\right) \cup Y: W \in \mathcal{P}\left(X_{1}\right)\right\} \\
& =\left\{Y^{\prime} \cup X_{1}\right\} \cup \bigcup_{i=2}^{|X|}\left\{\left(X_{1}-\left(W \cup\left\{x_{i}\right\}\right)\right) \cup Y^{\prime}: W \in \mathcal{P}\left(X_{i}\right)\right\} \cup\left\{\left(X-\left(W \cup\left\{x_{1}\right\}\right)\right) \cup Y: W \in \mathcal{P}\left(X_{1}\right)\right\} \\
& \left(Y^{\prime}=Y \cup\left\{x_{1}\right\}\right) \\
& =\left\{Y^{\prime} \cup Z\right\} \cup \bigcup_{j=1}^{|Z|}\left\{\left(Z-\left(W \cup\left\{z_{j}\right\}\right)\right) \cup Y^{\prime}: W \in \mathcal{P}\left(Z_{j}\right)\right\} \cup\left\{\left(X-\left(W \cup\left\{x_{1}\right\}\right)\right) \cup Y: W \in \mathcal{P}\left(X_{1}\right)\right\} \\
& \left(Z=Z_{0}=X_{1}, \ldots, Z_{|Z|}=X_{|X|}\right) \\
& =\left\{W \cup Y^{\prime}: W \in \mathcal{P}(Z)\right\} \cup\left\{\left(X-\left(W \cup\left\{x_{1}\right\}\right)\right) \cup Y: W \in \mathcal{P}\left(X_{1}\right)\right\} \\
& \text { (pela hipótese de indução) } \\
& =\left\{W \cup Y^{\prime}: W \in \mathcal{P}\left(X_{1}\right)\right\} \cup\left\{\left(X-\left(W \cup\left\{x_{1}\right\}\right)\right) \cup Y: W \in \mathcal{P}\left(X_{1}\right)\right\} \\
& \text { (uma vez que } X_{1}=Z \text { ) } \\
& =\left\{W \cup\left\{x_{1}\right\} \cup Y: W \in \mathcal{P}\left(X_{1}\right)\right\} \cup\left\{\left(X-\left(W \cup\left\{x_{1}\right\}\right)\right) \cup Y: W \in \mathcal{P}\left(X_{1}\right)\right\} \\
& \text { (uma vez que } Y^{\prime}=Y \cup\left\{x_{1}\right\} \text { ) } \\
& =\left\{W \cup\left\{x_{1}\right\} \cup Y: W \in \mathcal{P}\left(X_{1}\right)\right\} \cup\left\{\left(\left(X-\left\{x_{1}\right\}\right)-W\right) \cup Y: W \in \mathcal{P}\left(X_{1}\right)\right\} \\
& =\left\{W \cup\left\{x_{1}\right\} \cup Y: W \in \mathcal{P}\left(X_{1}\right)\right\} \cup\left\{\left(X_{1}-W\right) \cup Y: W \in \mathcal{P}\left(X_{1}\right)\right\} \\
& \text { (uma vez que } X_{1}=X-\left\{x_{1}\right\} \text { ) } \\
& =\left\{W \cup\left\{x_{1}\right\} \cup Y: W \in \mathcal{P}\left(X_{1}\right)\right\} \cup\left\{W \cup Y: W \in \mathcal{P}\left(X_{1}\right)\right\} \\
& =\{W \cup Y: W \in \mathcal{P}(X)\} \text {. }
\end{aligned}
$$

A aplicação recursiva da decomposição apresentada no lema 6.2.1 produz uma árvore $T^{\prime}$. Seja $T$ uma árvore construída através da aplicação recursiva da decomposição apresentada no lema 6.2.1. Se $V_{T^{\prime}}=V_{T}$ e:

$$
E_{T^{\prime}}=\left\{\left\{A^{c}, B^{c}\right\}:\{A, B\} \in E_{T}\right\},
$$

em que $A^{c}=X-A$ e $B^{c}=X-B$, então dizemos que $T^{\prime}$ é complementar a $T$. Observe que essa propriedade é comutativa, ou seja, que se $T$ é complementar a $T^{\prime}$ então $T^{\prime}$ é complementar a $T$; podemos dar ênfase a esta propriedade dizendo que $T$ e $T^{\prime}$ são complementares entre si.

A seguinte condição é suficiente para a produção de duas árvores complementares entre si.

Proposição 6.2.2. Sejam $X$ e $Y$ conjuntos finitos, $X$ não-vazio, e seja $\mathcal{X}$ uma cadeia $X_{0} \supseteq X_{1} \supseteq$ $\ldots \supseteq X_{|X|}$ tal que $X_{0}=X, X_{|X|}=\emptyset$ e $X_{i} \cup\left\{x_{i}\right\}=X_{i-1}$ para todo $0<i \leq|X|$. Sejam $T e$ $T^{\prime}$ árvores construídas através de aplicações recursivas das decomposições dos lemas 6.1.1 e 6.2.1, respectivamente, utilizando nessas decomposições $X, Y$ e $\mathcal{X} . T$ é complementar a $T^{\prime}$.

Demonstração. Esta prova é uma indução em $|X|$. Se $|X|=0$, então tanto $T$ quanto $T^{\prime}$ são árvores vazias, portanto com o mesmo número de vértices (nenhum) e com todas as suas arestas (nenhuma) complementares entre si. Dessa fora, $T$ é complementar a $T^{\prime}$.

Agora, consideremos quando $|X|>0$. Sejam $T$ e $T^{\prime}$ as árvores produzidas através de aplicações recursivas dos lemas 6.2.1 e 6.2.1. Como ambas as árvores são geradoras do grafo de Hasse do 
reticulado Booleano $(\mathcal{P}(X \cup Y), \subseteq)$, vale que $V_{T^{\prime}}=V_{T}$.

Falta mostrarmos que vale a condição:

$$
E_{T^{\prime}}=\left\{\left\{A^{c}, B^{c}\right\}:\{A, B\} \in E_{T}\right\} .
$$

Seja $\emptyset$ a raiz de $T$. Aplicando a decomposição do lema 6.1.1, para cada elemento $Y \cup\left\{x_{i}\right\}, x_{i} \in X, T$ possui o vértice $Y \cup\left\{x_{i}\right\}$ e a aresta $\left\{\emptyset, Y \cup\left\{x_{i}\right\}\right\}$. De forma análoga, seja $X$ a raiz de $T^{\prime}$. Aplicando a decomposição do lema 6.2.1, para cada elemento $X-\left(Y \cup\left\{x_{i}\right\}\right)=\left(Y \cup\left\{x_{i}\right\}\right)^{c}, x_{i} \in X, T$ possui o vértice $\left(Y \cup\left\{x_{i}\right\}\right)^{c}$ e a aresta $\left\{X,\left(Y \cup\left\{x_{i}\right\}\right)^{c}\right\}$. Observe que para cada uma dessas arestas $\left\{\emptyset, Y \cup\left\{x_{i}\right\}\right\}$ de $T$ existe uma aresta $\left\{X,\left(Y \cup\left\{x_{i}\right\}\right)^{c}\right\}=\left\{\emptyset^{c},\left(Y \cup\left\{x_{i}\right\}\right)^{c}\right\}$ de $T^{\prime}$. Além disso, por hipótese de indução, cada um desses vértices $Y \cup\left\{x_{i}\right\}$ de $T$ é raiz de uma sub-árvore que é complementar a uma sub-árvore cuja raiz é o vértice $\left(Y \cup\left\{x_{i}\right\}\right)^{c}$ de $T^{\prime}$. Portanto, $T$ é complementar a $T^{\prime}$.

Na figura 6.6 mostramos um exemplo de enumerações sobre um mesmo reticulado Booleano, utilizando aplicações recursivas das decomposições dos lemas 6.1.1 e 6.2.1 que produzem, respectivamente, as árvores $T$ e $T^{\prime}$. Como mostraremos adiante, o fato de $T$ ser complementar a $T^{\prime}$ será

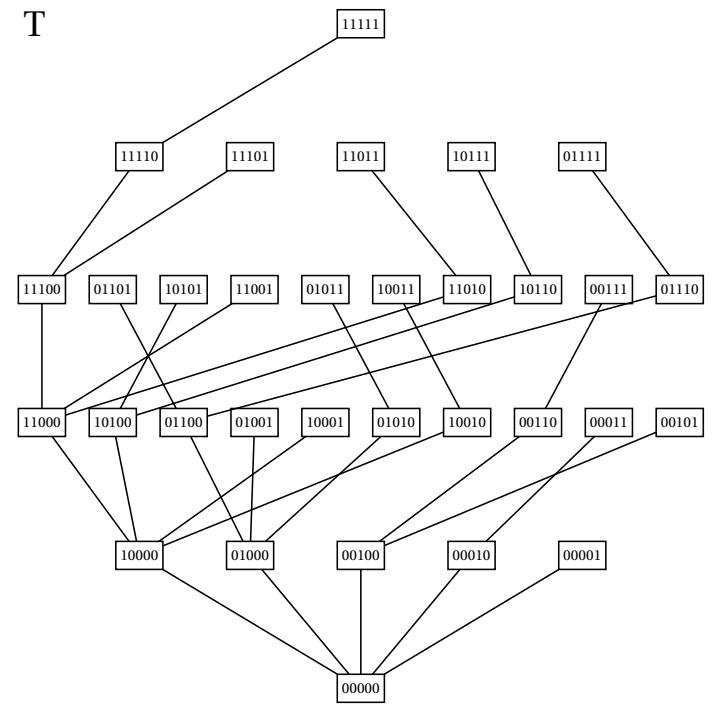

(a)

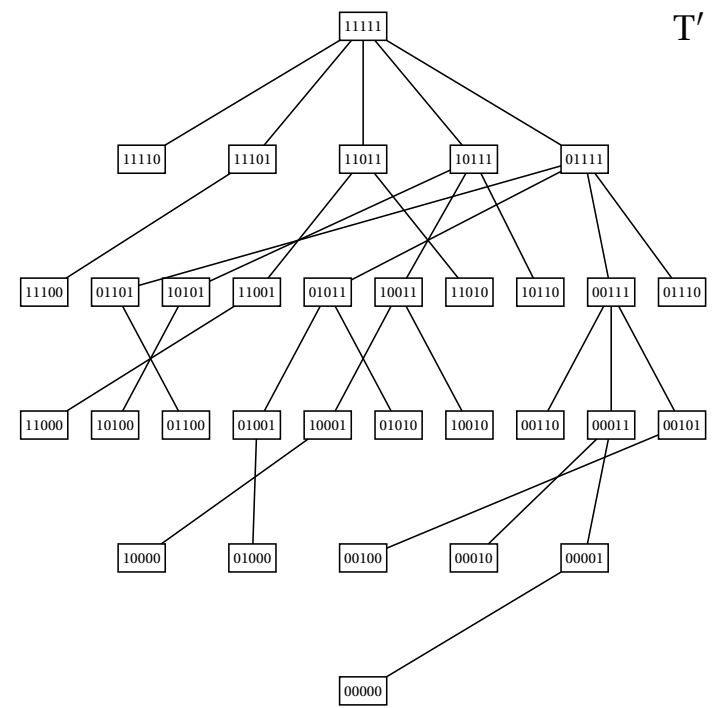

(b)

Figura 6.6: um exemplo de enumerações de um reticulado Booleano, utilizando aplicações recursivas das decomposições dos lemas 6.1.1 (figura 6.6(a)) e 6.2.1 (figura 6.6(b)). Observe que para qualquer aresta $\{X, Y\}$ na árvore $T$ existe uma aresta $\left\{X^{c}, Y^{c}\right\}$ na árvore $T^{\prime}$.

útil para efetuarmos podas no novo algoritmo.

O novo algoritmo utiliza como representação do espaço de busca duas árvores $T$ e $T^{\prime}$, tais que $T$ é complementar a $T^{\prime}$. Durante a execução do algoritmo, o mesmo sempre mantém a equivalência entre os espaços de busca correntes: ou seja, se o algoritmo poda de $T$ os vértices contidos no intervalo $[Y, X \cup Y]$, então ele também precisa podar de $T^{\prime}$ os vértices contidos nesse mesmo intervalo. Na figura 6.7 mostramos o resultado das podas das árvores $T$ e $T^{\prime}$ utilizando o intervalo [11100, $00011 \cup$ $11100]=[11100,11111]$. O resultado da eliminação dos vértices de $T^{\prime}$ contidos nesse intervalo é uma floresta, ou seja, um grafo que é composto por uma ou mais árvores. Portanto, o exemplo da figura nos mostra que, no novo algoritmo, o gerenciamento do espaço de busca corrente é feito por duas florestas $\mathcal{A}$ e $\mathcal{B}$, que são atualizadas a cada vez que ocorre uma poda.

Apresentaremos agora algumas regras que constituem o núcleo da dinâmica do novo algoritmo.

(i) Inicialização das florestas. Antes da primeira iteração, $\mathcal{A}=T$ e $\mathcal{B}=T^{\prime} ; T$ e $T^{\prime}$ possuem, respectivamente, as raízes 00000 e 11111 ; 
PRUNeD $\mathrm{T}$

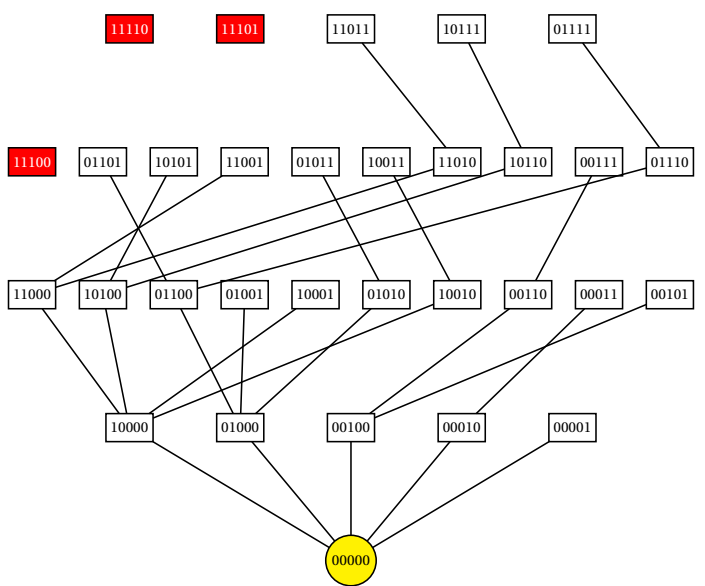

(a)
11111 PRUNed $T^{\prime}$

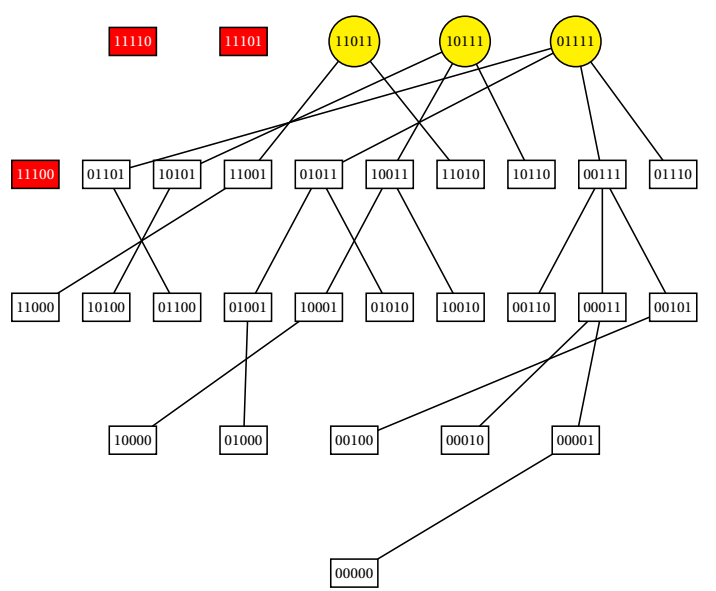

(b)

Figura 6.7: um exemplo de uma poda realizada na árvore $T$, no qual é eliminado do espaço de busca o intervalo [11100,11111] (figura 6.7(a)). A eliminação desse intervalo em $T^{\prime}$ produz uma floresta contendo três árvores (figura 6.7(b)). As raízes das árvores estão destacadas em amarelo.

Esta regra garante que o algoritmo inicialize com o espaço de busca completo e equivalente nas duas direções.

(ii) Percorrimento das florestas. Uma busca sempre se inicia em uma raiz de uma árvore, respeitando o esquema de enumeração;

(iii) Representação das florestas. Para qualquer raiz de árvore $R$, os filhos de $R$ são raízes de sub-árvores completas;

Uma consequência destas duas regras é que, durante a execução do algoritmo, basta guardar na memória as raízes das árvores e uma lista de seus respectivos filhos, pois os demais vértices são obtidos através do esquema de enumeração.

(iv) Gerenciamento das florestas. Durante uma iteração qualquer, um vértice que é verificado é ponta de um intervalo a ser podado nas florestas ou então torna-se uma raiz de uma nova árvore;

Como consequência desta regra, se dois vértices $A$ e $B$ de uma mesma árvore são adjacentes e são visitados durante a busca, então a aresta $\{A, B\}$ é eliminada dessa árvore.

(v) Poda das florestas. Podar um intervalo $[Y, X \cup Y]([Y-X, Y])$ na floresta $\mathcal{A}(\mathcal{B})$ significa eliminar de $\mathcal{A}(\mathcal{B})$ todos os vértices contidos na sub-árvore obtida através de aplicações recursivas da decomposição do lema 6.1.1 (6.2.1), utilizando $X$ e $Y$ na decomposição inicial. Além disso, tais vértices também precisam ser eliminados de $\mathcal{B}(\mathcal{A})$.

Falta agora definir uma maneira sistemática para cumprir a regra (v), isto é, dada a ocorrência de poda na floresta $\mathcal{A}(\mathcal{B})$ de um intervalo $[Y, X \cup Y]([Y-X, Y])$, como realizar uma poda na floresta $\mathcal{B}(\mathcal{A})$ de uma forma mais eficiente do que a verificação exaustiva de todos os elementos deste intervalo. Os resultados a seguir nos mostram uma maneira de se fazer isso, aproveitando-se do fato de $T$ ser complementar a $T^{\prime}$.

Proposição 6.2.3. Sejam $T$ e $T^{\prime}$ duas árvores tais que $T$ é complementar a $T^{\prime}$. Eliminar de $T^{\prime}$ os vértices contidos no intervalo $[Y, X \cup Y]$ é equivalente a eliminar de $T^{\prime}$ todos os vértices do caminho 
$P$ em $T^{\prime}$ com extremos $Y$ e $X \cup Y$ e também todos os vértices de $T^{\prime}$ contidos em intervalos $[Y, B]$ tais que $B$ contém propriamente $Y$ e $B$ é adjacente inferior a um vértice de $P$.

Demonstração. Iniciamos a demonstração observando que a sub-árvore $T_{Y}$ definida em $T$ pelo intervalo $[Y, X \cup Y]$ é complementar a sub-árvore $T_{Y}^{\prime}$ definida pelo mesmo intervalo em $T^{\prime}$; portanto, existe um caminho $P$ em $T^{\prime}$ com extremos $Y$ e $X \cup Y$. Além disso, observe que todo intervalo $[Y, B]$ tal que $B$ contém propriamente $Y$ e $B$ é adjacente inferior a um vértice de $P$ está contido propriamente no intervalo $[Y, X \cup Y]$ (portanto, não são podados vértices fora de $T_{Y}^{\prime}$ ). Finalmente, observe que não existe vértice de $T_{Y}^{\prime}$ que não seja coberto por um desses intervalos e que não esteja em $P$.

O resultado da proposição 6.2.3 também vale para o reticulado Booleano $(\mathcal{P}(S), \supseteq)$.

Proposição 6.2.4. Sejam $T$ e $T^{\prime}$ duas árvores tais que $T$ é complementar a $T^{\prime}$. Eliminar de $T$ os vértices contidos no intervalo $[Y-X, Y]$ é equivalente a eliminar de $T$ todos os vértices do caminho $P$ em $T$ com extremos $Y-X$ e $Y$ e também todos os vértices de $T$ contidos em intervalos $[A, Y]$ tais que $A$ é contido propriamente em $Y$ e A é adjacente superior a um vértice de $P$.

Demonstração. Aplicando o princípio da dualidade, o resultado da proposição 6.2.3 pode ser estendido para o reticulado Booleano $(\mathcal{P}(S), \supseteq)$.

Agora apresentaremos uma simulação do algoritmo PoseT-ForeST-SEARCH (PFS), um branchand-bound ótimo para abordar o problema U-curve, que controla o espaço de busca através de duas florestas $\mathcal{A}$ e $\mathcal{B}$; tais florestas são inicialmente definidas como sendo, respectivamente, as árvores $T$ e $T^{\prime}, T$ complementar a $T^{\prime}$ (regra (i)). A cada nova iteração, as duas florestas são redefinidas através de sucessivas podas (i.e., através de remoção de vértices e de arestas contidos em intervalos do reticulado Booleano). Cada iteração deste algoritmo é dividida em duas fases:

- na primeira fase, uma das florestas é escolhida e o algoritmo percorre uma de suas árvores. O percorrimento é um caminho $P$ na árvore escolhida, sendo a raiz desta árvore um dos extremos de $P$ (regra (ii)). $P$ é aumentado com novos vértices, até que ocorra uma das seguintes situações:

- seja visitada uma folha $Y$; neste caso, a mesma é removida da floresta, o que é equivalente a remover da floresta todos os vértices contidos no intervalo $[Y, Y]$;

- seja visitado um vértice $Y$ cujo custo seja maior que o custo do elemento que o precede neste caminho; neste caso, são removidos da floresta todos os vértices contidos no intervalo $[Y, X \cup Y]$ (se a floresta da iteração for $\mathcal{A}$ ) ou no intervalo $[Y-X, Y]$ (se a floresta da iteração for $\mathcal{B})$.

Após o percorrimento, seguindo a regra (iv), todos os vértices que foram visitados e que não foram eliminados da floresta tornam-se raízes de árvores.

- na segunda fase, seguindo a regra (v), a floresta que não foi escolhida é atualizada, podando da mesma o mesmo intervalo que foi podado na floresta que foi escolhida na iteração.

Nas figuras 6.8-6.14 mostramos uma simulação do algoritmo PFS, feita a partir da instância mostrada na figura 6.1(a). As florestas $\mathcal{A}$ e $\mathcal{B}$ são inicializadas com as raízes 00000 e 11111 , respectivamente. PFS é simulado da seguinte maneira: 


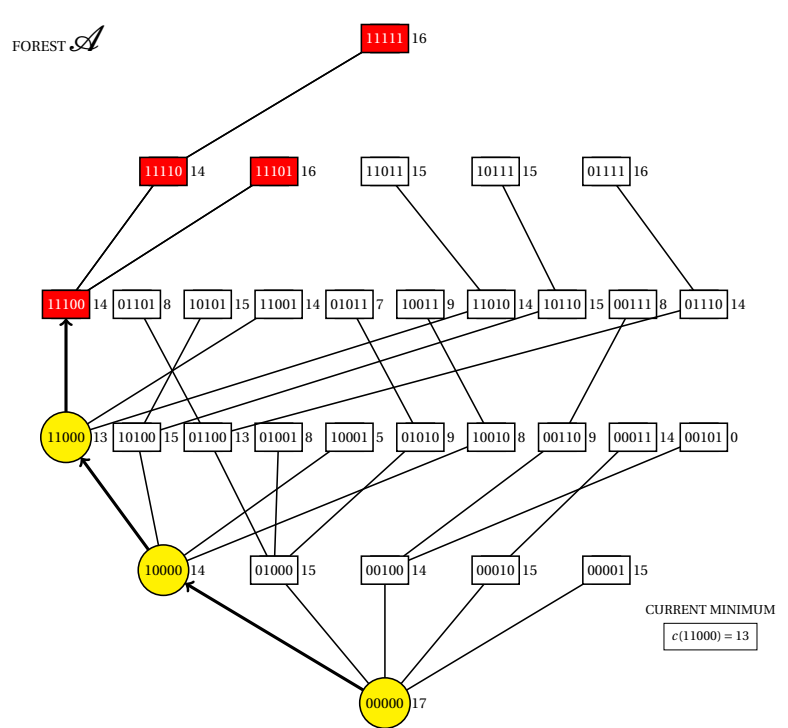

(a)

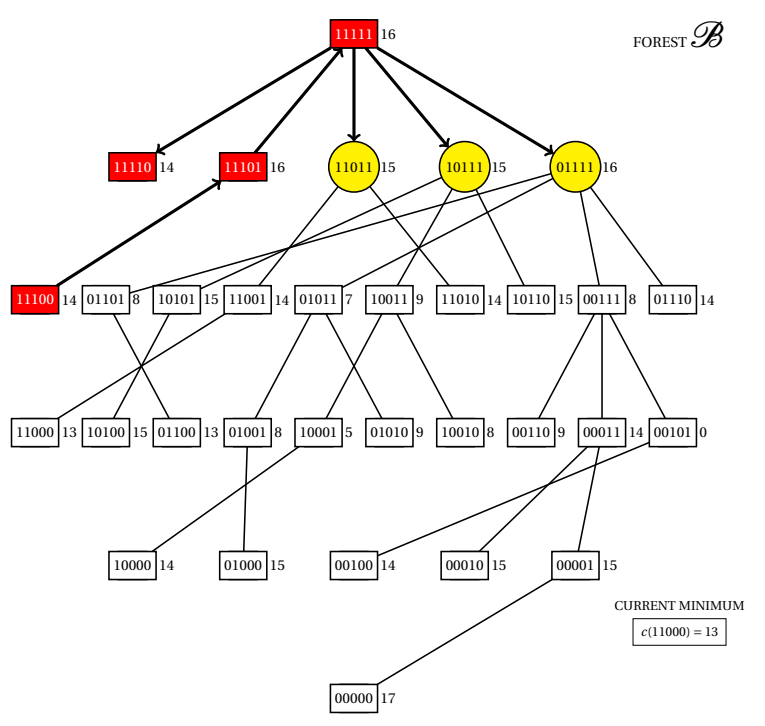

(c)

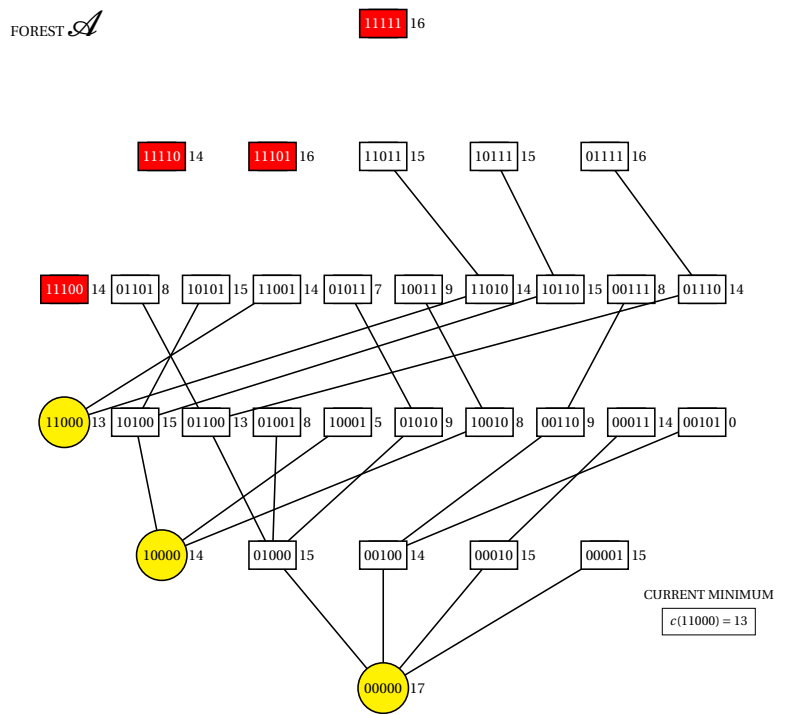

(b)

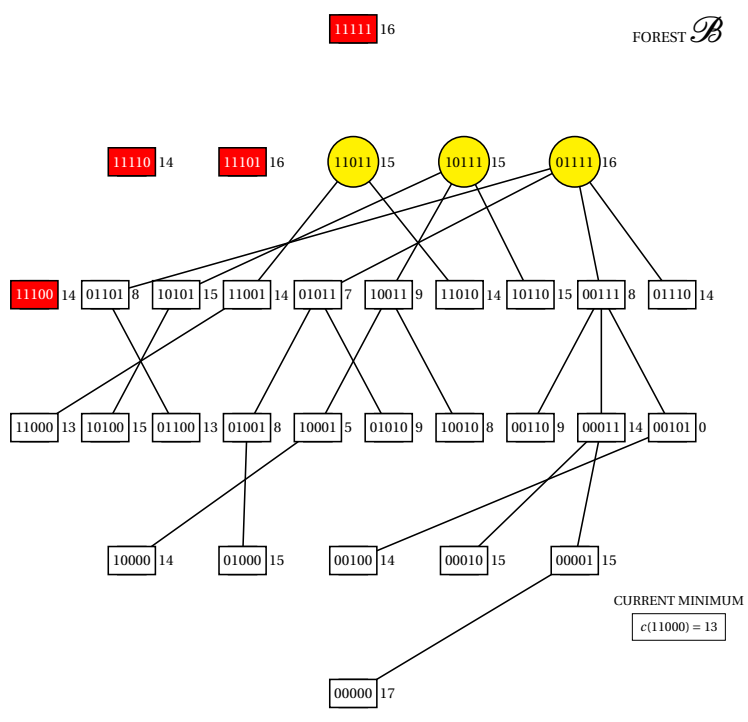

(d)

Figura 6.8: uma simulação de POSET-FOREST-SEARCH (PFS), um algoritmo branch-and-bound para abordar o problema U-curve.

- Figura 6.8(a): iteração sobre a floresta $\mathcal{A}$. O algoritmo ramifica até o vértice 11100; como $c(11100)>c(11000)$, os vértices contidos no intervalo [11100,11111] são eliminados de $\mathcal{A}$;

- Figura 6.8(b): pela regra (iv), os vértices 00000, 10000 e 11000 tornam-se raízes de árvores;

- Figura 6.8(c): atualização da floresta $\mathcal{B}$, para respeitar a regra (v). Como os vértices contidos no intervalo $[11100,11111]$ foram eliminados da floresta $\mathcal{A}$, pela proposição 6.2 .3 são eliminados de $\mathcal{B}$ os vértices que pertencem a um caminho $P$ em $T^{\prime}$ com extremos 11100 e 11111 (11100, 11101 e 11111), assim como os vértices que contêm propriamente 11100 e são adjacentes inferiores a um vértice de $P$ (11110; os elementos 11011, 10111 e 01111 são visitados, porém eles não contêm propriamente 11100). Finalmente, como 11111 é raiz de árvore em $\mathcal{B}$, a sua remoção não viola a regra (iii);

- Figura 6.8(d): pela regra (iv), os vértices 11011, 10111 e 01111 tornam-se raízes de árvores; 
1111116

FOREST $\mathscr{B}$

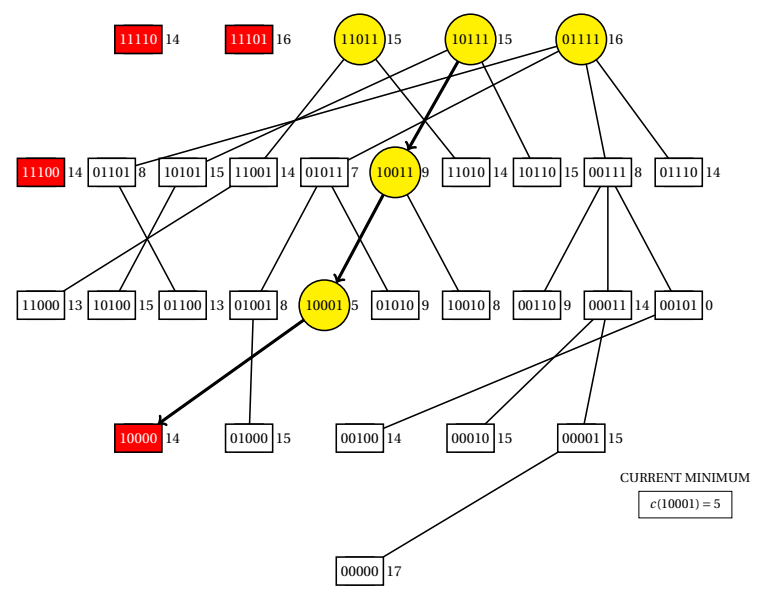

(a)

FOREST $\mathscr{A}$

1111116

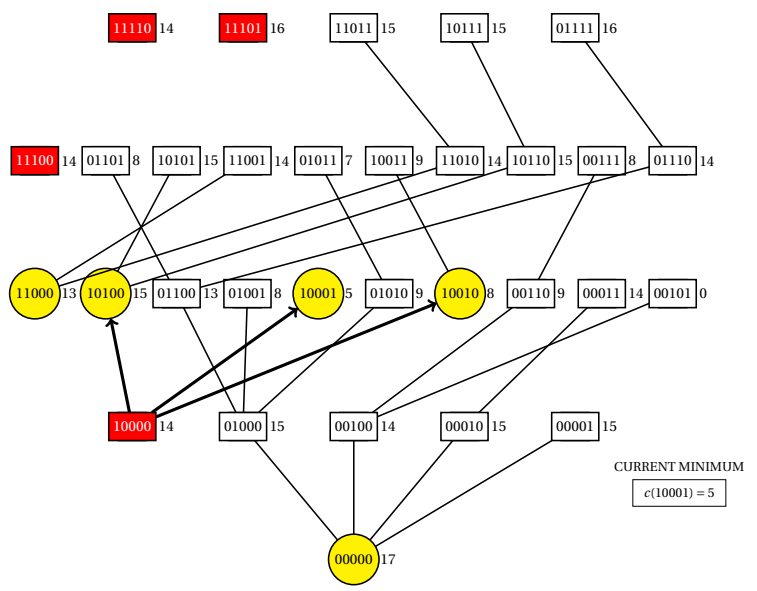

(c)

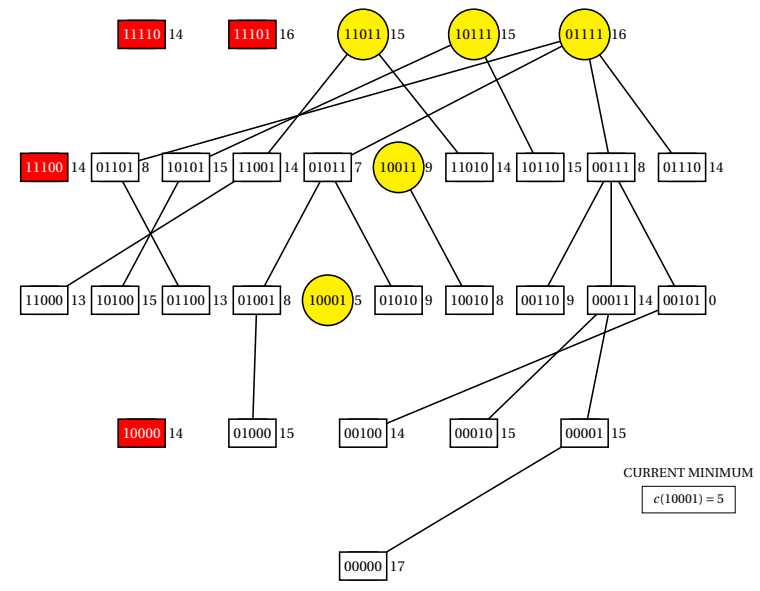

(b)
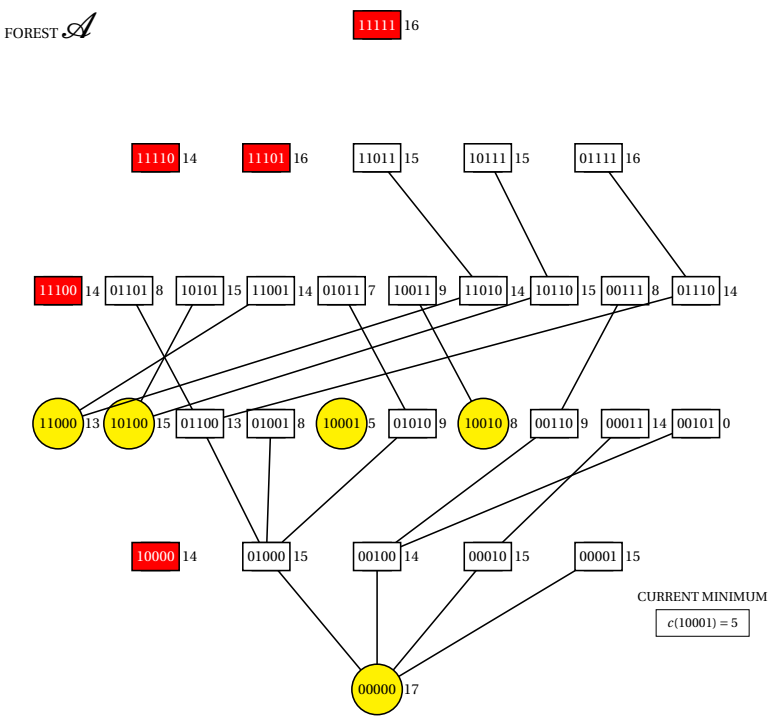

$(\mathrm{d})$

Figura 6.9: continuação da simulação da figura 6.8.

- Figura 6.9(a): iteração sobre a floresta $\mathcal{B}$. O algoritmo escolhe uma das raízes (10111) e ramifica até o vértice 10000; uma vez que $c(10000)>c(10001)$, os vértices contidos no intervalo $[10000,10000]$ são eliminados de $\mathcal{B}$;

- Figura 6.9(b): pela regra (iv), os vértices 10001 e 10011 tornam-se raízes de árvores;

- Figura 6.9(c): atualização da floresta $\mathcal{A}$, para respeitar a regra (v). Como os vértices contidos no intervalo $[10000,10000]$ foram eliminados de $\mathcal{B}$, pela proposição 6.2 .4 são eliminados de $\mathcal{A}$ os vértices que pertencem a um caminho $P$ em $T$ com extremos 10000 e 10000 (10000), e também os que são contidos propriamente em 10000 e que são adjacentes superiores a um vértice de $P$ (os elementos 10100, 10001 e 10010 são visitados, porém eles não são contidos propriamente em 10000). Finalmente, como 10000 é raiz de árvore em $\mathcal{A}$, a sua remoção não viola a regra (iii);

- Figura 6.9(d): pela regra (iv), os vértices 10100, 10001 e 10010 tornam-se raízes de árvores; 
FOREST $\mathscr{A}$

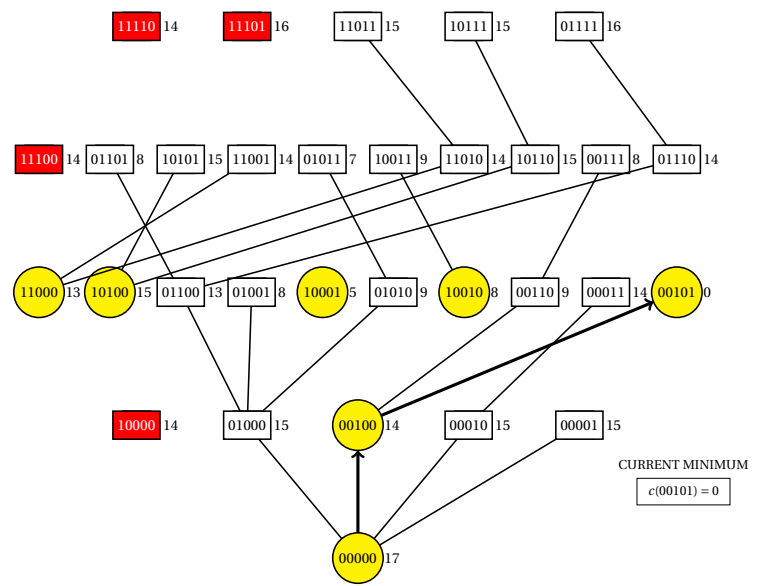

(a)

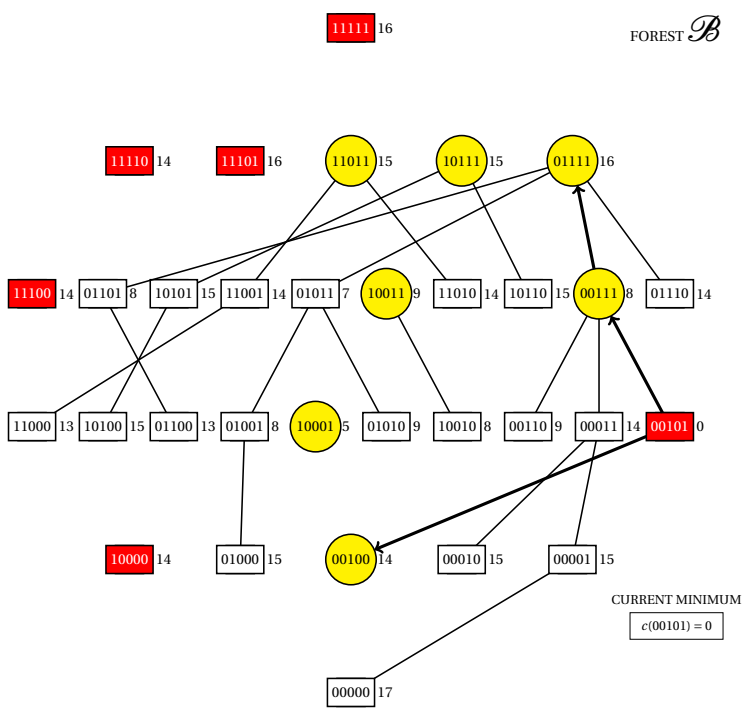

(c)
FOREST $\mathscr{A}$

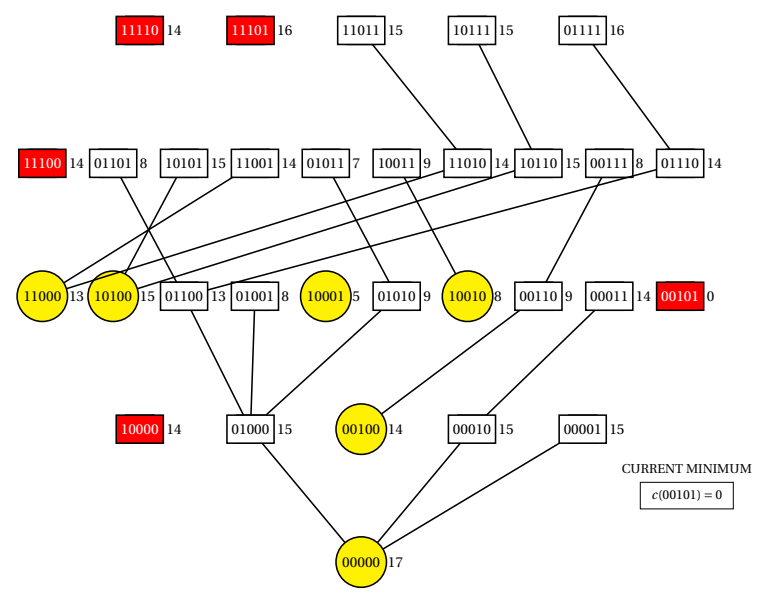

(b)

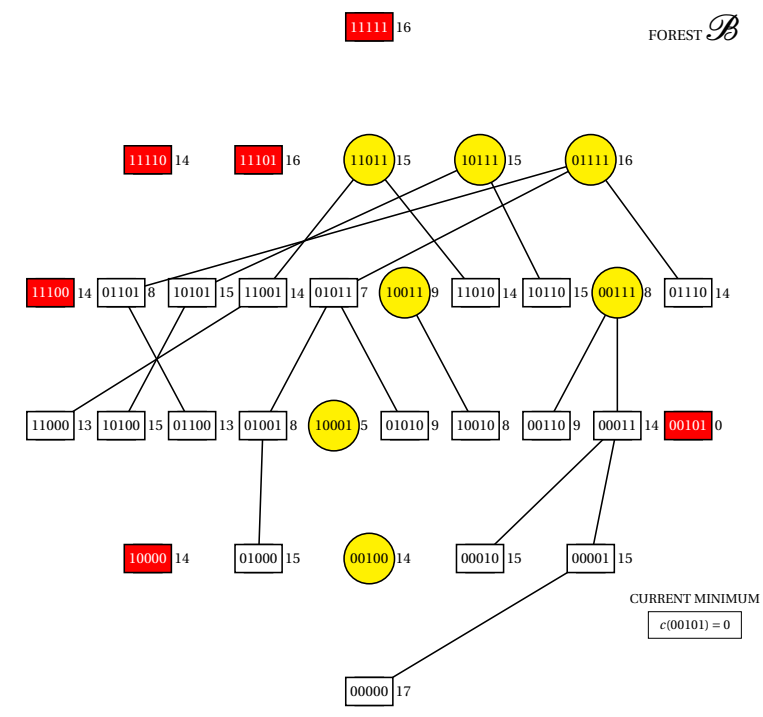

(d)

Figura 6.10: continuação da simulação da figura 6.9.

- Figura 6.10(a): iteração sobre a floresta $\mathcal{A}$. O algoritmo escolhe uma das raízes (00000) e ramifica até o vértice 00101;

- Figura 6.10(b): pela regra (iv), 00101 é raiz de uma árvore trivial, logo ele é removido de $\mathcal{A}$;

- Figura 6.10(c): atualização da floresta $\mathcal{B}$, para respeitar a regra (v). Como os vértices contidos no intervalo [00101,00101] foram eliminados da floresta $\mathcal{A}$, pela proposição 6.2 .3 são eliminados de $\mathcal{B}$ os vértices que pertencem a um caminho $P$ em $T^{\prime}$ com extremos 00101 e 00101 (00101), assim como os vértices que contêm propriamente 00101 e são adjacentes inferiores a um vértice de $P$ (o elemento 00100 é visitado, porém ele não contém propriamente 00101). Finalmente, como 00101 não é raiz de árvore em $\mathcal{B}$, a sua remoção viola a regra (iii); para corrigir isto é percorrido um caminho na árvore corrente até que seja encontrada a raiz da mesma (o caminho percorrido contém os vértices 00101, 00111 e 01111);

- Figura 6.10(d): pela regra (iv), os vértices 00100 e 00111 tornam-se raízes de árvores; 
Forest $\mathscr{A}$

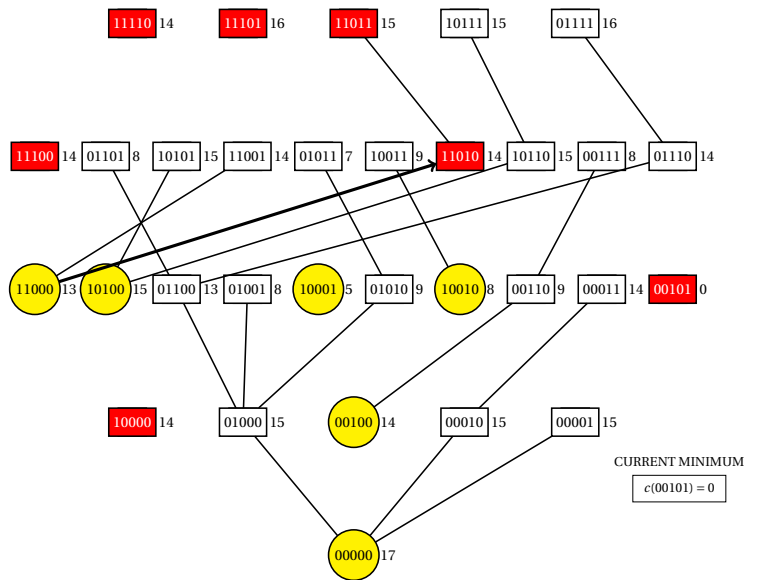

(a)

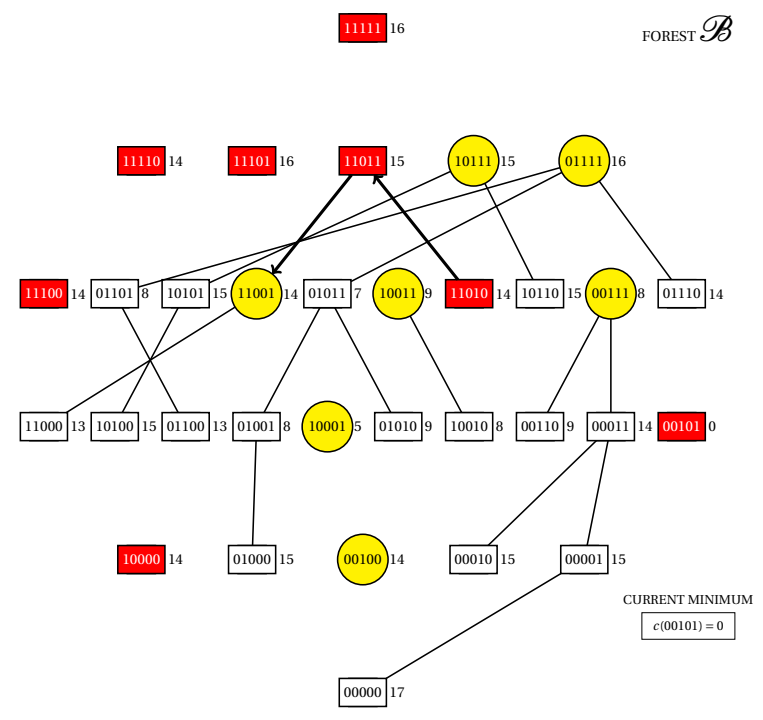

(c)
FoRest $\mathscr{A}$

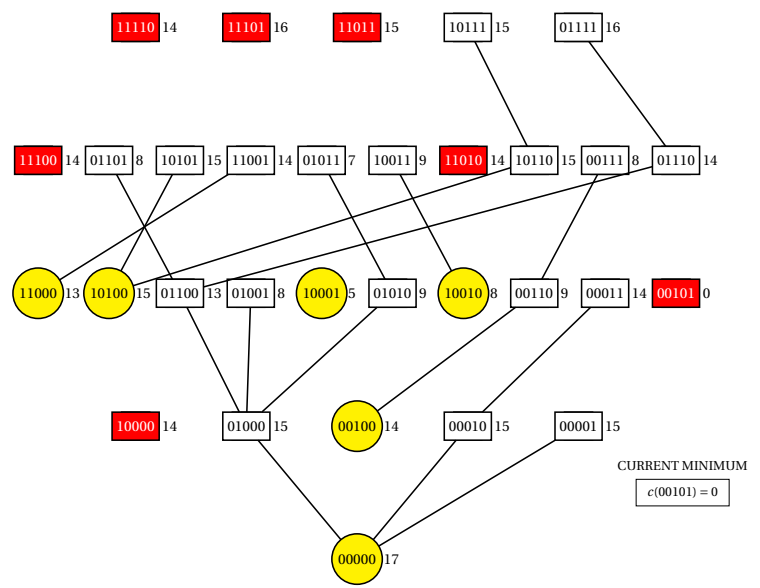

(b)

1111116

FOREST $\mathscr{B}$

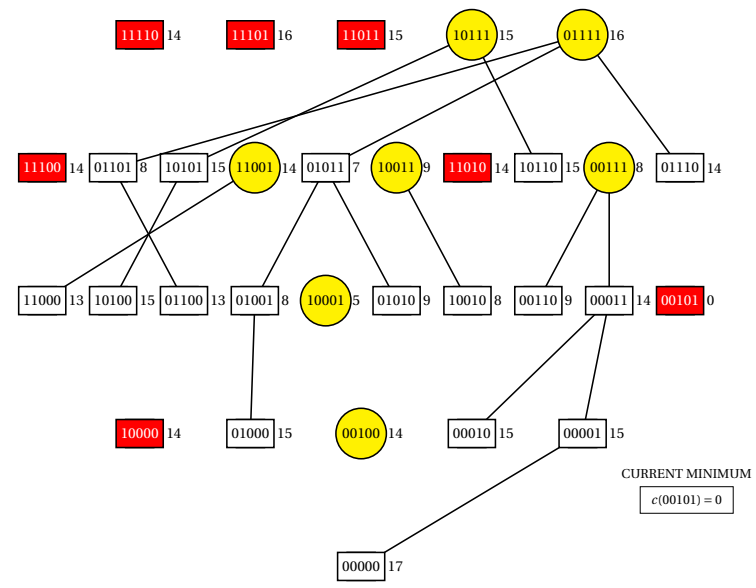

(d)

Figura 6.11: continuação da simulação da figura 6.10.

- Figura 6.11(a): iteração sobre a floresta $\mathcal{A}$. O algoritmo escolhe uma das raízes (11000) e ramifica até o vértice 11010; uma vez que $c(11010)>c(11000)$, os vértices contidos no intervalo $[11010,11011]$ são eliminados de $\mathcal{A}$;

- Figura 6.11(b): a floresta $\mathcal{A}$, após sua atualização;

- Figura 6.11(c): atualização da floresta $\mathcal{B}$, para respeitar a regra (v). Como os vértices contidos no intervalo $[11010,11011]$ foram eliminados da floresta $\mathcal{A}$, pela proposição 6.2 .3 são eliminados de $\mathcal{B}$ os vértices que pertencem a um caminho $P$ em $T^{\prime}$ com extremos 11010 e 11011 (11010, e 11011), assim como os vértices que contêm propriamente 11010 e são adjacentes inferiores a um vértice de $P$ (o elemento 11001 é visitado, porém ele não contém propriamente 11010). Finalmente, como 11011 é raiz de árvore em $\mathcal{B}$, a sua remoção não viola a regra (iii);

- Figura 6.11(d): pela regra (iv), o vértice 11001 torna-se raiz de árvore; 
$111111^{16}$

FOREST $\mathscr{B}$

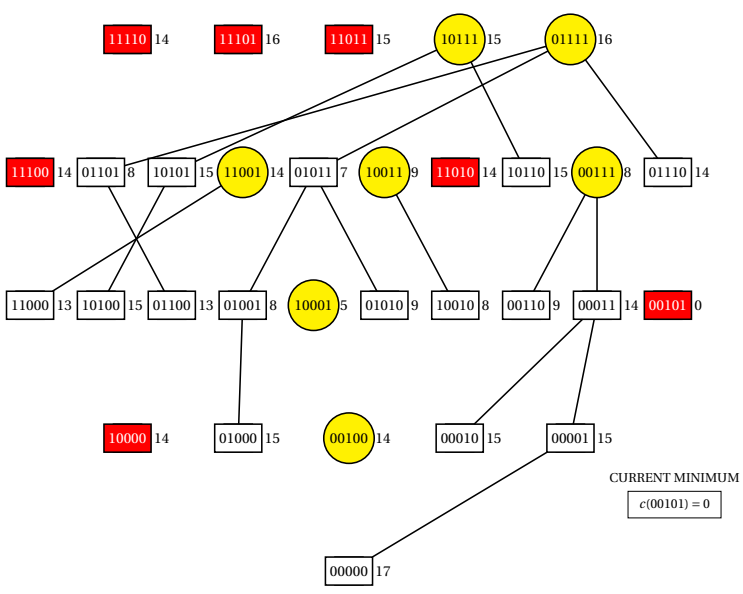

(a)

FOREST $\mathscr{A}$

1111116

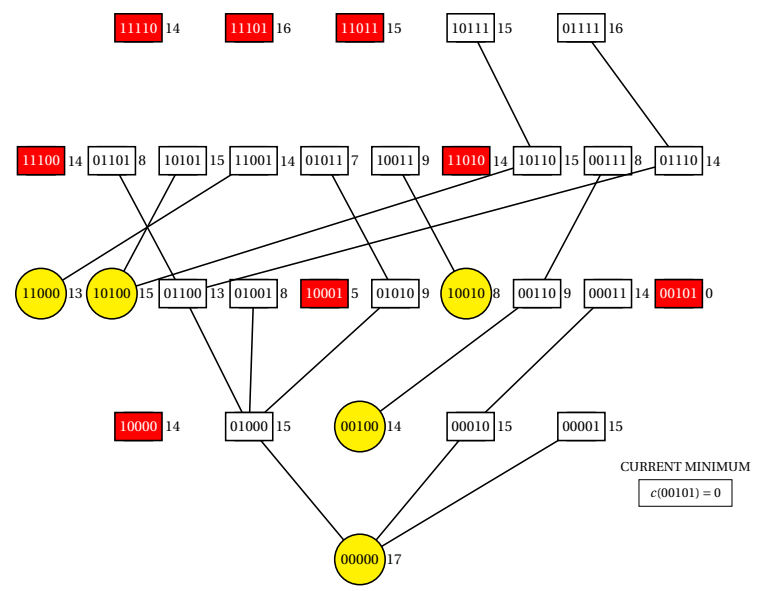

(c)

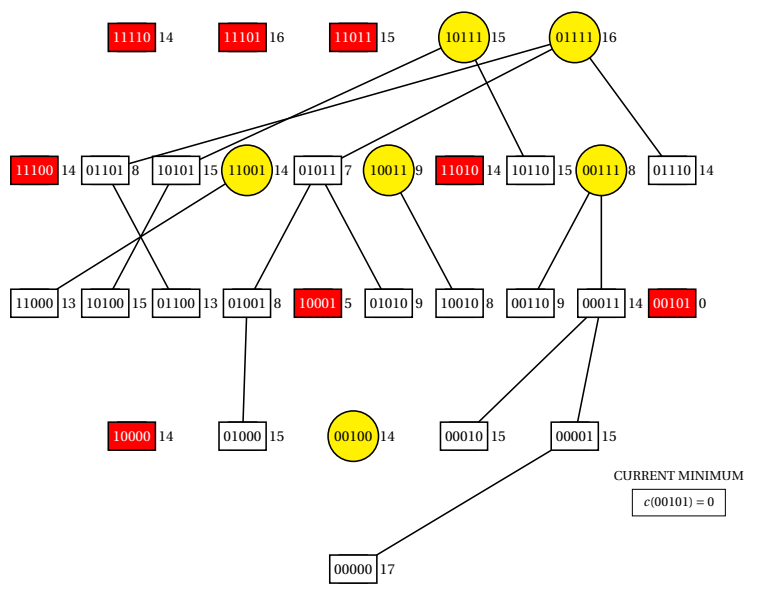

(b)
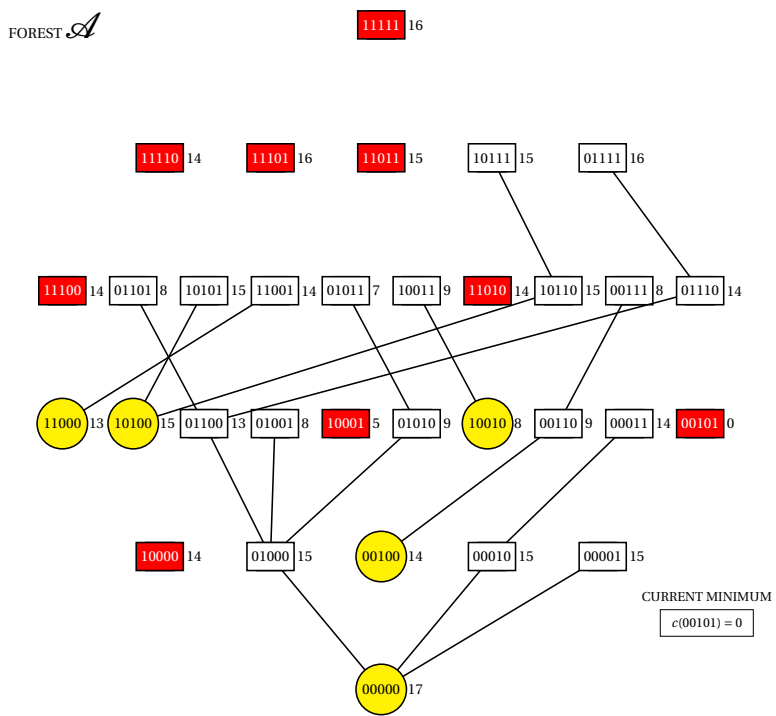

(d)

Figura 6.12: continuação da simulação da figura 6.11.

- Figura 6.12(a): iteração sobre a floresta $\mathcal{B}$. O algoritmo escolhe uma das raízes (10001) e ramifica até o vértice 10001;

- Figura 6.12(b): 10001 torna-se raiz de uma árvore trivial, portanto ele é removido de $\mathcal{B}$;

- Figura 6.12(c): atualização da floresta $\mathcal{A}$, para respeitar a regra (v). Como os vértices contidos no intervalo $[10001,10001]$ foram eliminados da floresta $\mathcal{B}$, pela proposição 6.2 .4 são eliminados de $\mathcal{A}$ os vértices que pertencem a um caminho $P$ em $T$ com extremos 10001 e 10001 (10001), assim como os vértices que são contidos propriamente em 10001 e que são adjacentes superiores a um vértice de $P$ (neste caso, nenhum); Finalmente, como 10001 é raiz de árvore em $\mathcal{A}$, a sua remoção não viola a regra (iii);

- Figura 6.12(d): a floresta $\mathcal{A}$, após sua atualização; 


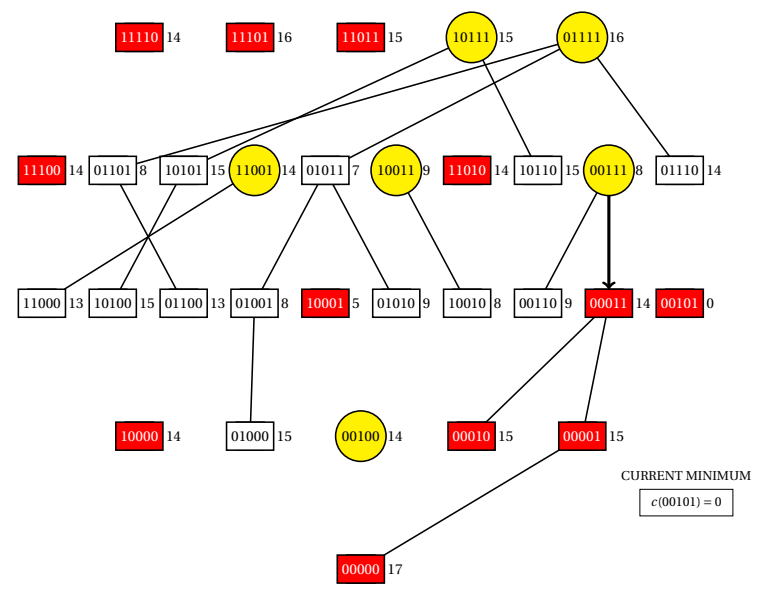

(a)

FOREST $\mathscr{A}$

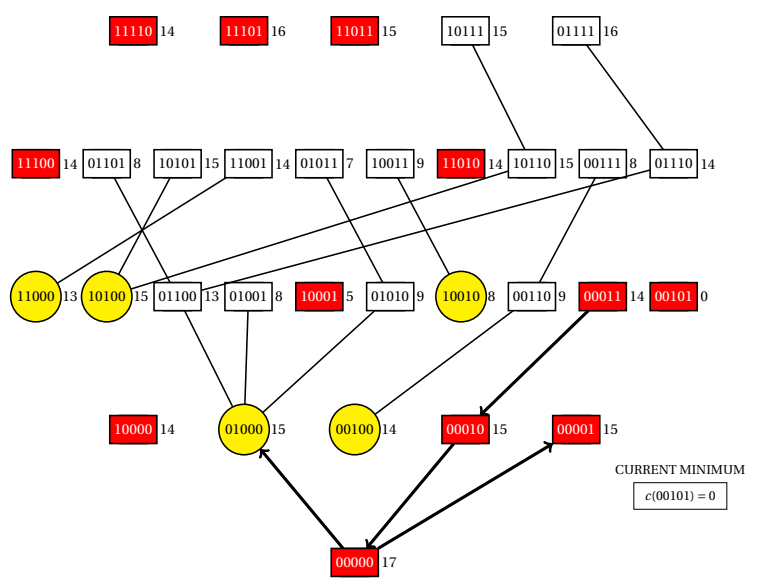

(c)

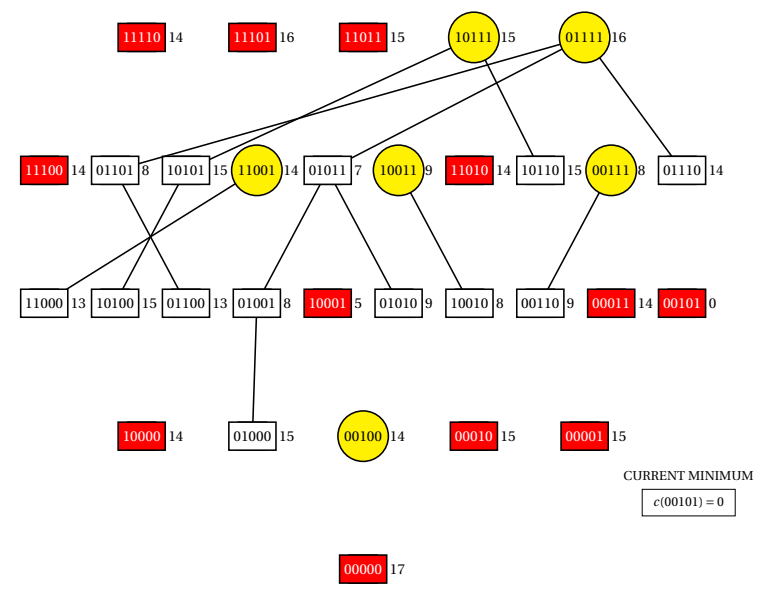

(b)

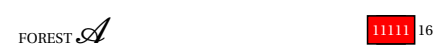

$(\mathrm{d})$

Figura 6.13: continuação da simulação da figura 6.12.

- Figura 6.13(a): iteração sobre a floresta $\mathcal{B}$. O algoritmo escolhe uma das raízes (00111) e ramifica até o vértice 00011; uma vez que $c(00011)>c(00111)$, os vértices contidos no intervalo $[00000,00011]$ são eliminados de $\mathcal{B}$;

- Figura 6.13(b): a floresta $\mathcal{B}$, após sua atualização;

- Figura 6.13(c): atualização da floresta $\mathcal{A}$, para respeitar a regra (v). Como os vértices contidos no intervalo [00000, 00011] foram eliminados da floresta $\mathcal{B}$, pela proposição 6.2 .4 são eliminados de $\mathcal{A}$ os vértices que pertencem a um caminho $P$ em $T$ com extremos 00011 e 00000 (00011, 00010 e 00000), assim como os vértices que são contidos propriamente em 00011 e que são adjacentes superiores a um vértice de $P(00001$; o elemento 01000 é visitado, porém ele não é contido propriamente em 00011); Finalmente, como 00000 é raiz de árvore em $\mathcal{A}$, a sua remoção não viola a regra (iii);

- Figura 6.13(d): pela regra (iv), o vértice 01000 torna-se raiz de árvore; 
FOREST $\mathscr{A}$

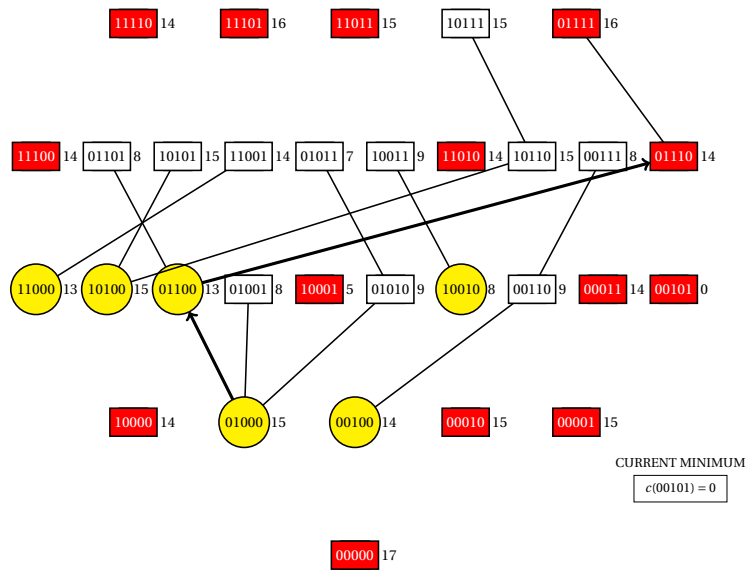

(a)

1111116

FOREST $\mathscr{B}$

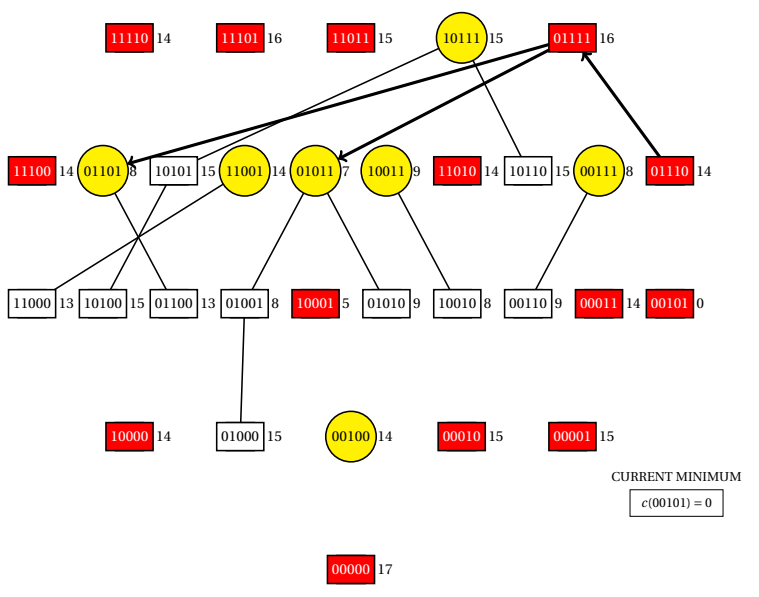

(c)
FOREST $\mathscr{A}$

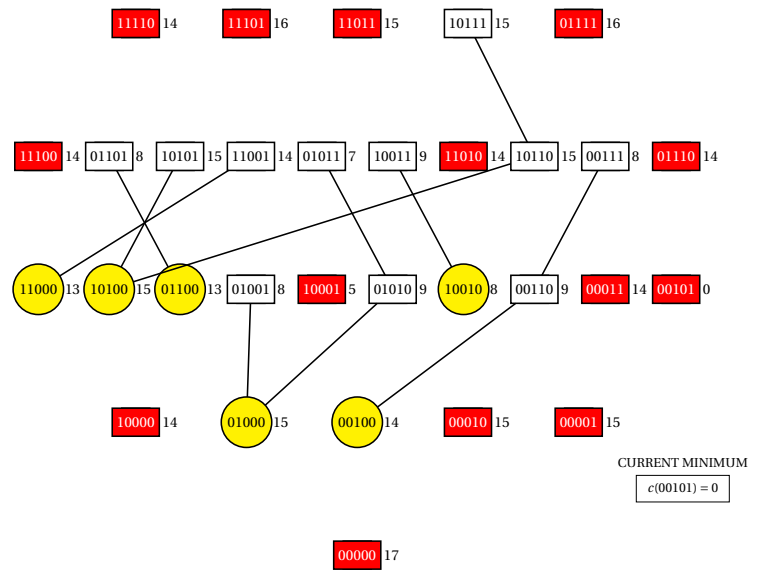

(b)

1111116

FOREST $\mathscr{B}$

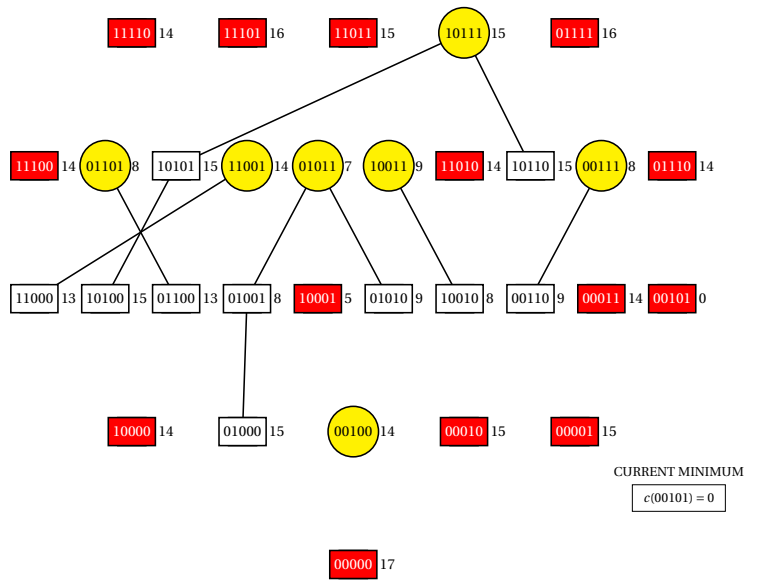

(d)

Figura 6.14: continuação da simulação da figura 6.13.

- Figura 6.14(a): iteração sobre a floresta $\mathcal{A}$. O algoritmo escolhe uma das raízes (01000) e ramifica até o vértice 01110; uma vez que $c(01110)>c(01000)$, os vértices contidos no intervalo [01110, 01111] são eliminados de $\mathcal{A}$;

- Figura 6.14(b): pela regra (iv), o vértice 01100 torna-se raiz de árvore;

- Figura 6.14(c): atualização da floresta $\mathcal{B}$, para respeitar a regra (v). Como os vértices contidos no intervalo $[01110,01111]$ foram eliminados de $\mathcal{A}$, pela proposição 6.2 .3 são eliminados de $\mathcal{B}$ os vértices que pertencem a um caminho $P$ em $T^{\prime}$ com extremos 01110 e 01111 (01110 e 01111), assim como os vértices que contêm propriamente 01110 e que são adjacentes inferiores a um vértice de $P$ (os elementos 01101 e 01011 são visitados, porém eles não contêm propriamente 01110); Finalmente, como 01111 é raiz de árvore em $\mathcal{B}$, a sua remoção não viola a regra (iii);

- Figura 6.14(d): pela regra (iv), os vértices 01101 e 01011 tornam-se raízes de árvores. 
A partir do estado mostrado na figura 6.14(d), o algoritmo itera até que as florestas tornem-se vazias (i.e., que todo o espaço de busca seja esgotado). A seguir, apresentaremos o pseudo-código e uma análise de complexidade de tempo do algoritmo PFS e de suas sub-rotinas.

\subsubsection{Pseudo-código e complexidade de tempo}

Seja $S$ um conjunto finito e não vazio. Uma sequência $S_{s e q}$ de $S$ é uma lista ordenada $\left\langle s_{1}, \ldots, s_{n}\right\rangle$ dos elementos de $S$. Seja $s$ um elemento de $S$. O índice de $s$ em $S_{\text {seq }}$ pode ser obtido através de uma bijeção $f$ de $S$ para $\{1, \ldots, n\}$; por exemplo, se $s$ é o terceiro elemento da sequência cuja bijeção é $f$, então $f(s)=3$ e $f^{-1}(3)=s$. Suporemos que o consumo de tempo de bijeções desse tipo seja de $O(1)$ unidades de tempo. Durante as iterações do algoritmo, $S_{\text {seq }}$ será utilizada para controlar os esquemas de enumeração tanto da floresta $\mathcal{A}$ quanto da floresta $\mathcal{B}$, assim satisfazendo a condição suficiente da proposição 6.2.2 para que $T$ e $T^{\prime}$ sejam complementares entre si.

Descrevemos agora as estruturas de dados que utilizaremos para implementar as florestas $\mathcal{A}$ e $\mathcal{B}$. Um nó $\mathbf{N}$ é uma estrutura contendo quatro campos, dos quais os dois primeiros mapeiam para um elemento de $\mathcal{P}(S)$, o terceiro mapeia para um inteiro e o último mapeia para um número real:

- "vertex" representa um vértice da floresta $\mathcal{A}(\mathcal{B})$;

- "adjacent" representa a topologia de um vértice em uma floresta. Se $\mathbf{N}[$ adjacent $]$ é vazio, então $\mathbf{N}[$ vertex $]$ é uma folha na floresta $\mathcal{A}(\mathcal{B}) ;$

- "leftmost" armazena o índice $i, 1 \leq i \leq n+1$, do primeiro elemento da sequência $\left\langle s_{1}, \ldots, s_{n}\right\rangle$ a partir do qual, pelo esquema de enumeração, a busca pode visitar elementos adjacentes a $\mathbf{N}[$ vertex $]$ em $\mathcal{A}(\mathcal{B})$ através da operação $\mathbf{N}[$ vertex $] \cup\left\{s_{i}\right\}\left(\mathbf{N}[\right.$ vertex $\left.]-\left\{s_{i}\right\}\right)$; se $i=n+1$, então $\mathbf{N}[$ vertex $]$ é uma folha na árvore geradora $T\left(T^{\prime}\right)$ do reticulado Booleano;

- "cost" armazena o custo de $\mathbf{N}[$ vertex], o qual é computado utilizando-se a função custo $c$.

Daqui em diante, suporemos que qualquer coleção de nós $\mathcal{F}$ é implementada utilizando uma árvore binária de busca balanceada (e.g., árvores rubro-negras [GS78]). Portanto, a complexidade de uma busca em uma coleção é $O(n \log |\mathcal{F}|)$, de uma inserção é $O(n \log |\mathcal{F}|)$ e de uma remoção é $O(n \log |\mathcal{F}|)$. Apresentaremos agora o pseudo-código do algoritmo PFS, que está dividido em programa principal e duas sub-rotinas, que executam, respectivamente, a primeira fase (percorrimento e poda de uma floresta) e a segunda fase (poda da outra floresta); para todos eles apresentaremos descrição e análise de complexidade de tempo.

\section{Percorrimento de um caminho}

Apresentaremos agora uma sub-rotina que realiza a primeira fase do algoritmo, para o caso do percorrimento ser feito sobre a floresta $\mathcal{A}$; o caso do percorrimento ser feito sobre a floresta $\mathcal{B}$ é dual a este.

Descrição de algoritmo. LOWER-Forest-BrAnch recebe duas coleções de nós não-vazias $\mathcal{F}_{\mathcal{A}}$ e $\mathcal{F}_{\mathcal{B}}$, uma função custo $c$ decomponível em curvas em U e uma bijeção $f$ dos elementos de $S$ para $\{1, \ldots, n\}$. Esta sub-rotina percorre um caminho $P$ que se inicia na raiz de uma das árvores da floresta $\mathcal{A}$, até que atinja um vértice $\mathbf{N}[$ vertex] que seja uma folha ou cujo custo seja maior que o custo do elemento que o precede neste caminho; neste caso, são removidos de $\mathcal{A}$ todos os vértices contidos no intervalo

$$
\left[\mathbf{N}[\text { vertex }], \mathbf{N}[\text { vertex }] \cup \bigcup_{e \in \mathbf{N}[\text { adjacent }]}\{e\}\right]
$$

Finalmente, esta sub-rotina devolve uma coleção $\mathcal{M}$ dos vértices do caminho $P$, o nó $\mathbf{N}$ e também a coleção atualizada $\mathcal{F}_{\mathcal{A}}$. 
LOWER-Forest-Branch $\left(\mathcal{F}_{\mathcal{A}}, \mathcal{F}_{\mathcal{B}}, c, f\right)$

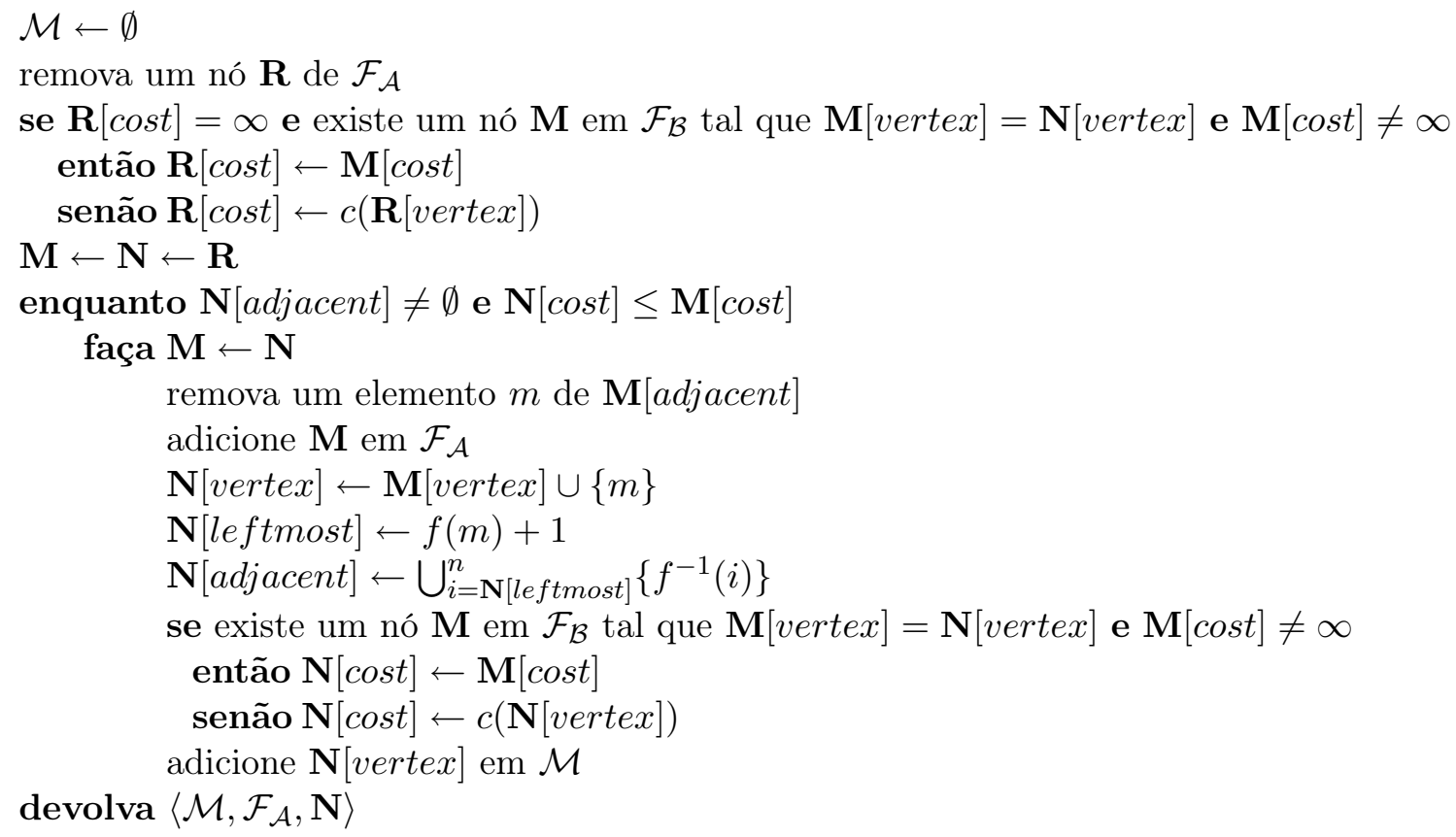

Durante a simulação apresentada a partir da figura 6.8, esta sub-rotina é equivalente às execuções da primeira fase do algoritmo exibidas nas figuras 6.8(a)-6.8(b) (com raiz $\mathbf{R}[$ vertex $]=$ 00000), 6.10(a)-6.10(b) $(\mathbf{R}[$ vertex $]=00000), 6.11(\mathrm{a})-6.11(\mathrm{~b})(\mathbf{R}[$ vertex $]=11000)$ e 6.14(a)$6.14(\mathrm{~b})(\mathbf{R}[$ vertex $]=01000)$.

Análise de complexidade de tempo. A remoção de um nó $\mathbf{R}$ na linha 2 consome $O\left(n \log \left|\mathcal{F}_{\mathcal{A}}\right|\right)$ unidades de tempo.

O laço enquanto nas linhas 7-17 itera no máximo $n+1$ vezes. Em cada iteração deste laço, todas as operações consomem $O(n)$ unidades de tempo, com exceção da inserção na linha 10, que consome $O\left(n \log \left|\mathcal{F}_{\mathcal{A}}\right|\right)$ unidades de tempo, das buscas nas linhas 3 e 14 , que requerem $O\left(n \log \left|\mathcal{F}_{\mathcal{B}}\right|\right)$ unidades de tempo cada, e do cálculo da função custo na linha 16, que exige $O(f(n))$ unidades de tempo. Dessa forma, o consumo de tempo total do laço das linhas 7-17 é de $O\left(n\left(n \log \left|\mathcal{F}_{\mathcal{A}}\right|+n \log \left|\mathcal{F}_{\mathcal{B}}\right|+f(n)\right)\right)$ unidades de tempo.

Portanto, Lower-Forest-Branch requer $O\left((n f(n))+n^{2}\left(\log \left|\mathcal{F}_{\mathcal{A}}\right|+\log \left|\mathcal{F}_{\mathcal{B}}\right|\right)\right)$ unidades de tempo.

Algoritmo dual. UPPER-Forest-BRAnCH recebe duas coleções de nós não-vazias $\mathcal{F}_{\mathcal{A}}$ e $\mathcal{F}_{\mathcal{B}}$, uma função custo $c$ decomponível em curvas em U e uma bijeção $f$ dos elementos de $S$ para $\{1, \ldots, n\}$. Esta sub-rotina percorre um caminho $P$ que se inicia na raiz de uma das árvores da floresta $\mathcal{B}$, até que atinja um vértice $\mathbf{N}[$ vertex $]$ que seja uma folha ou cujo custo seja maior que o custo do elemento que o precede neste caminho; neste caso, são removidos de $\mathcal{B}$ todos os vértices contidos no intervalo

$$
\left[\mathbf{N}[\text { vertex }]-\bigcup_{e \in \mathbf{N}[\text { adjacent }]}\{e\}, \mathbf{N}[\text { vertex }]\right] .
$$

Finalmente, esta sub-rotina devolve uma coleção $\mathcal{M}$ dos vértices do caminho $P$, o nó $\mathbf{N}$ e também a coleção atualizada $\mathcal{F}_{\mathcal{B}}$.

\section{Poda da floresta não-percorrida}

Apresentaremos agora três sub-rotinas. A primeira sub-rotina, dados dois vértices $M$ e $Y$ da floresta $\mathcal{B}$, cria árvores cujas raízes são os filhos de $M$ que não contêm $Y$. A segunda sub-rotina, dado 
um vértice $M$ da floresta $\mathcal{B}$ tal que $M$ não é raiz de árvore, faz uma busca pela raiz da árvore que contém $M$. Já a terceira sub-rotina, que faz uso das duas sub-rotinas anteriores, realiza a segunda fase do algoritmo para o caso da atualização ser feita sobre a floresta $\mathcal{B}$; o caso da atualização ser feita sobre a floresta $\mathcal{A}$ é dual a este.

Descrição de algoritmo. SEARCh-Lower-Children recebe dois conjuntos $M$ e $Y, Y \subseteq M$, um nó $\mathbf{M}$, uma coleção de nós $\mathcal{F}_{\mathcal{B}}$ e uma bijeção $f$ dos elementos de $S$ para $\{1, \ldots, n\}$. Esta sub-rotina supõe que se o vértice $M$ da floresta $\mathcal{B}$ ainda não foi visitado, então $\mathbf{M}=$ NIL; caso contrário, $\mathbf{M}[$ vertex $]=M$. Esta sub-rotina verifica as condições da proposição 6.2.3 para todo vértice $B$ adjacente inferior ao vértice $M$ : ou seja, se $B$ contém $Y$, então $B$ é removido da floresta $\mathcal{B}$. Se $B$ não contém $Y$ e $B$ não foi visitado ou removido da floresta $\mathcal{B}$, então um nó $\mathbf{B}$ tal que $\mathbf{B}[$ vertex $]=B$ é criado e adicionado na coleção de nós $\mathcal{F}_{\mathcal{B}}$. Finalmente, esta sub-rotina devolve a coleção de nós atualizada $\mathcal{F}_{\mathcal{B}}$.

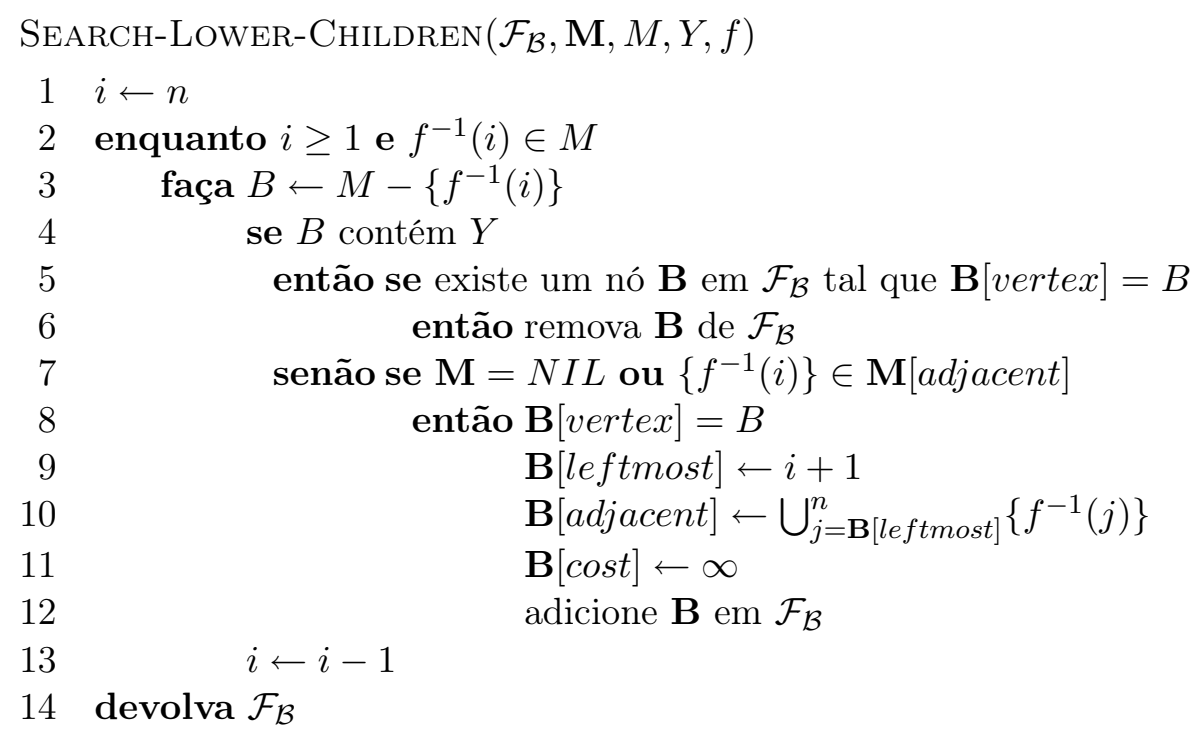

Durante a simulação apresentada a partir da figura 6.8, esta sub-rotina é equivalente às buscas por filhos feitas nas figuras 6.8(c) (três chamadas, com $Y=11100$ e $M=11100, Y=11100$ e $M=11101, Y=11100$ e $M=11111), 6.10(\mathrm{c})(Y=00101$ e $M=00101), 6.11(\mathrm{c})-6.11(\mathrm{~d})(\mathrm{duas}$ chamadas, $Y=11010$ e $M=11010, Y=11010$ e $M=11011)$ e $6.14(\mathrm{c})-6.14(\mathrm{~d})$ (duas chamadas, $Y=01110$ e $M=01110, Y=01110$ e $M=01111)$.

Análise de complexidade de tempo. O laço enquanto nas linhas 2-13 itera no máximo $n+1$ vezes. Em cada iteração deste laço, todas as operações consomem $O(n)$ unidades de tempo, com exceção da busca e da inserção nas linhas 5 e 12 , respectivamente, que requerem $O\left(n \log \left|\mathcal{F}_{\mathcal{B}}\right|\right)$ unidades de tempo cada. Dessa forma, o consumo de tempo total do laço das linhas 2-13 é de $O\left(n\left(n \log \left|\mathcal{F}_{\mathcal{B}}\right|\right)\right)$ unidades de tempo.

Portanto, Search-Lower-Children requer $O\left(n^{2} \log \left|\mathcal{F}_{\mathcal{B}}\right|\right)$ unidades de tempo.

Algoritmo dual. SeArch-Upper-Children recebe dois conjuntos $M$ e $Y, M \subseteq Y$, um nó M, uma coleção de nós $\mathcal{F}_{\mathcal{A}}$ e uma bijeção $f$ dos elementos de $S$ para $\{1, \ldots, n\}$. Esta sub-rotina supõe que se o vértice $M$ da floresta $\mathcal{A}$ ainda não foi visitado, então $\mathbf{M}=N I L$; caso contrário, $\mathbf{M}[$ vertex $]=M$. Esta sub-rotina verifica as condições da proposição 6.2 .4 para todo vértice $A$ adjacente superior ao vértice $M$ : ou seja, se $A$ é contido em $Y$, então $A$ é removido da floresta $\mathcal{A}$. Se $A$ não é contido em $Y$ e $A$ não foi visitado ou removido da floresta $\mathcal{A}$, então um nó $\mathbf{A}$ tal que $\mathbf{A}[$ vertex $]=A$ é criado e adicionado na coleção de nós $\mathcal{F}_{\mathcal{A}}$. Finalmente, esta sub-rotina devolve a coleção de nós atualizada $\mathcal{F}_{\mathcal{A}}$. 
Descrição de algoritmo. SEARCh-UpPeR-Root recebe uma coleção de nós $\mathcal{F}_{\mathcal{B}}$, uma bijeção $f$ dos elementos de $S$ para $\{1, \ldots, n\}$ e um elemento $M$. Esta sub-rotina supõe a existência de uma raiz $R$ em $\mathcal{B}$ tal que $M \subseteq R$; ela percorre um caminho em $\mathcal{B}$, com início em $M$, até encontrar uma raiz de árvore. Durante o percorrimento, esta sub-rotina cria as raízes de árvores necessárias para evitar a violação da regra (iv). Finalmente, esta sub-rotina devolve a coleção de nós atualizada $\mathcal{F}_{\mathcal{B}}$.

$\operatorname{SEARCH-UPPER-\operatorname {RoOT}}\left(\mathcal{F}_{\mathcal{B}}, M, f\right)$

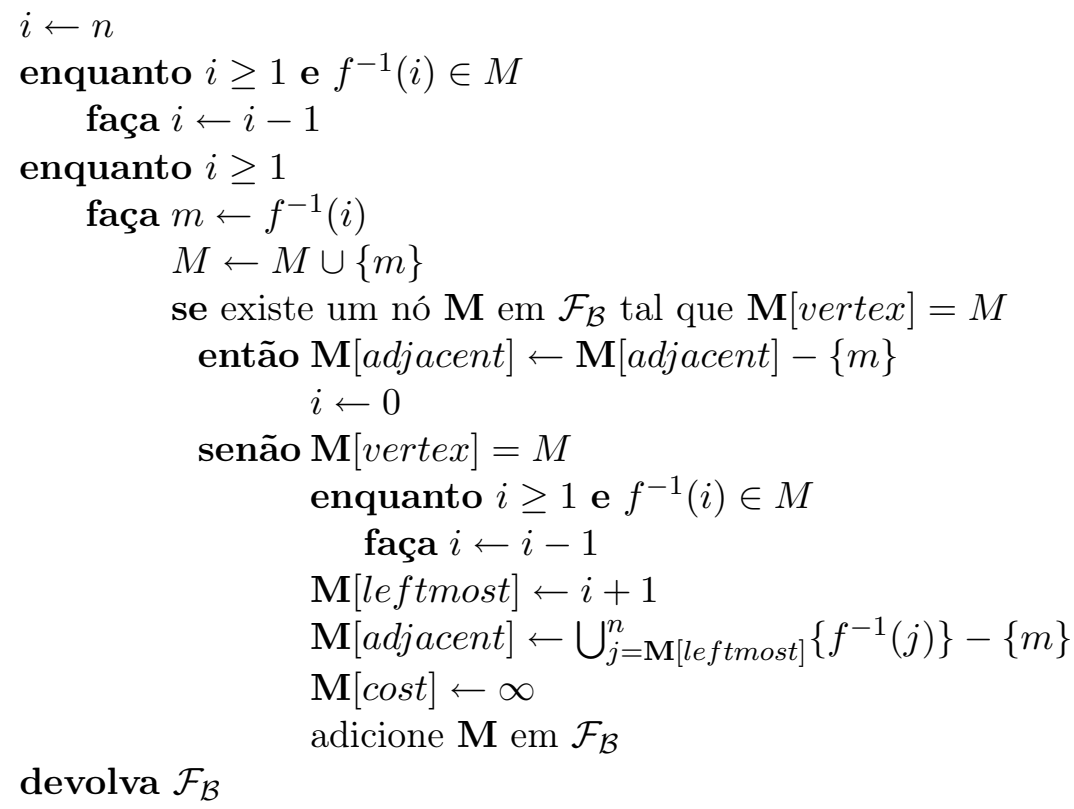

Durante a simulação apresentada a partir da figura 6.8, esta sub-rotina é equivalente à busca por uma raiz feita na figura $6.10(\mathrm{c})(M=00101)$.

Análise de complexidade de tempo. Os laços enquanto nas linhas 2-3 e 4-16 iteram no máximo $n+1$ vezes cada. Em cada iteração deste primeiro laço, todas as operações exigem $O(1)$ unidades de tempo. Portanto o primeiro laço requer $O(n)$ unidades de tempo. Em cada iteração deste segundo laço, todas as operações consomem $O(1)$ unidades de tempo, com exceção da busca e da inserção nas linhas 7 e 16 , respectivamente, que requerem $O\left(n \log \left|\mathcal{F}_{\mathcal{B}}\right|\right)$ unidades de tempo cada. Dessa forma, o consumo de tempo total do laço das linhas 4-16 é de $O\left(n\left(n \log \left|\mathcal{F}_{\mathcal{B}}\right|\right)\right)$ unidades de tempo.

Portanto, SeArch-Upper-Root requer $O\left(n^{2} \log \left|\mathcal{F}_{\mathcal{B}}\right|\right)$ unidades de tempo.

Algoritmo dual. SEArCh-Lower-Root recebe uma coleção de nós $\mathcal{F}_{\mathcal{A}}$, uma bijeção $f$ dos elementos de $S$ para $\{1, \ldots, n\}$ e um elemento $M$. Esta sub-rotina supõe a existência de uma raiz $R$ em $\mathcal{A}$ tal que $R \subseteq M$; ela percorre um caminho em $\mathcal{A}$, com início em $M$, até encontrar uma raiz de árvore. Durante o percorrimento, esta sub-rotina cria as raízes de árvores necessárias para evitar a violação da regra (iv). Finalmente, esta sub-rotina devolve a coleção de nós atualizada $\mathcal{F}_{\mathcal{A}}$.

Descrição de algoritmo. UpPer-Forest-Pruning recebe um nó $\mathbf{N}$, uma coleção de nós $\mathcal{F}_{\mathcal{B}}$ e uma bijeção $f$ dos elementos de $S$ para $\{1, \ldots, n\}$. Esta sub-rotina poda da floresta $\mathcal{A}$ todos os vértices contidos no intervalo

$$
\left[\mathbf{N}[\text { vertex }], \mathbf{N}[\text { vertex }] \cup \bigcup_{e \in \mathbf{N}[\text { adjacent }]}\{e\}\right],
$$

de acordo com a regra (v). Durante a poda, esta sub-rotina cria as raízes de árvores necessárias para evitar a violação da regra (iv). Finalmente, esta sub-rotina devolve a coleção de nós atualizada $\mathcal{F}_{\mathcal{A}}$. 
$\operatorname{Upper-Forest-Pruning}\left(\mathcal{F}_{\mathcal{A}}, \mathcal{F}_{\mathcal{B}}, \mathbf{N}, f\right)$

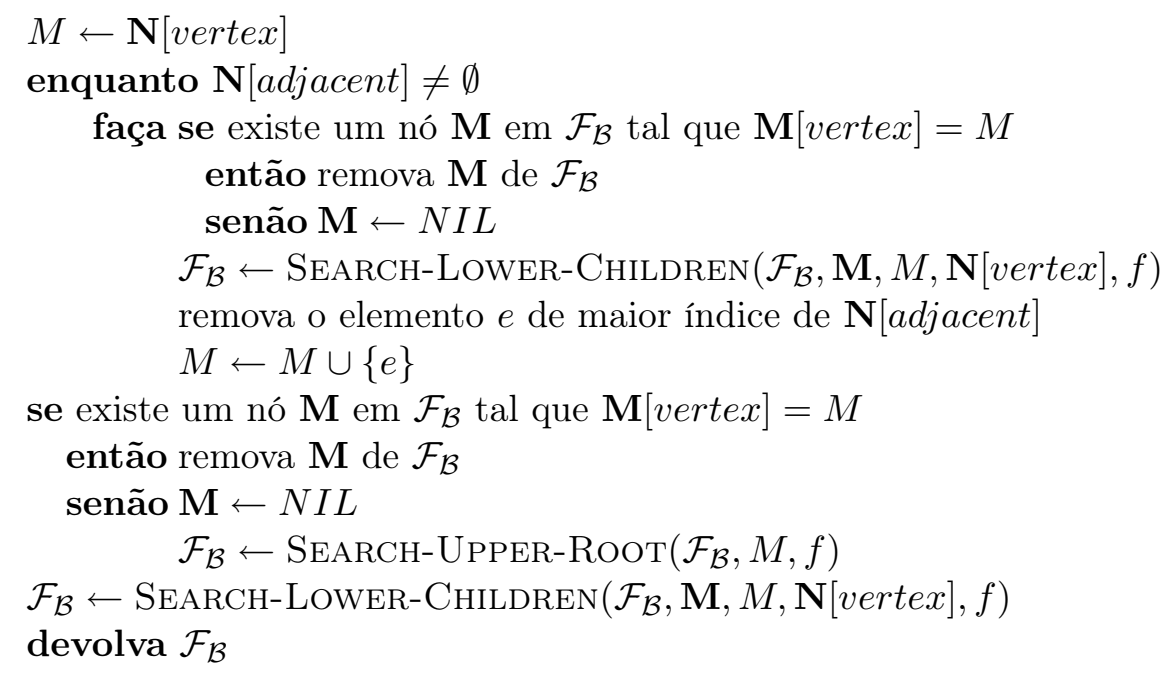

Durante a simulação apresentada a partir da figura 6.8, esta sub-rotina é equivalente às atualizações da floresta $\mathcal{B}$ feitas nas figuras 6.8(c)-6.8(d) (com $\mathbf{N}[$ vertex $]=11100$ ) e $6.10(\mathrm{c})-6.10(\mathrm{~d})$ $(\mathbf{N}[$ vertex $]=00101), 6.11(\mathrm{c})-6.11(\mathrm{~d})(\mathbf{N}[$ vertex $]=11000)$ e $6.14(\mathrm{c})-6.14(\mathrm{~d})(\mathbf{N}[$ vertex $]=01000)$.

Análise de complexidade de tempo. O laço enquanto nas linhas 2-8 itera no máximo $n+1$ vezes. Em cada iteração deste laço, todas as operações consomem $O(n)$ unidades de tempo, com exceção da busca e da remoção nas linhas 3 e 4 , respectivamente, que consomem $O\left(n \log \left|\mathcal{F}_{\mathcal{B}}\right|\right)$ unidades de tempo cada. Como cada chamada de SEARCH-LOWER-ChILDREN na linha 6 querer $O\left(n^{2} \log \left|\mathcal{F}_{\mathcal{B}}\right|\right)$ unidades de tempo, o consumo de tempo total do laço das linhas 2-8 é de $O\left(n^{3} \log \left|\mathcal{F}_{\mathcal{B}}\right|\right)$ unidades de tempo.

A chamada da busca e da remoção nas linhas 9 e 10, respectivamente, consomem $O\left(n \log \left|\mathcal{F}_{\mathcal{B}}\right|\right)$ unidades de tempo cada. Finalmente, Search-Lower-Children e SEarch-Upper-Root são chamadas nas linhas 13 e 12 , respectivamente, e quererem $O\left(n^{2} \log \left|\mathcal{F}_{\mathcal{B}}\right|\right)$ unidades de tempo cada.

Portanto, Upper-Forest-Pruning requer $O\left(n^{3} \log \left|\mathcal{F}_{\mathcal{B}}\right|\right)$ unidades de tempo.

Algoritmo dual. Lower-Forest-Pruning recebe um nó $\mathbf{N}$, uma coleção de nós $\mathcal{F}_{\mathcal{A}}$ e uma bijeção $f$ dos elementos de $S$ para $\{1, \ldots, n\}$. Esta sub-rotina poda da floresta $\mathcal{B}$ todos os vértices contidos no intervalo

$$
\left[\mathbf{N}[\text { vertex }]-\bigcup_{e \in \mathbf{N}[\text { adjacent }]}\{e\}, \mathbf{N}[\text { vertex }]\right]
$$

de acordo com a regra (v). Durante a poda, esta sub-rotina cria as raízes de árvores necessárias para evitar a violação da regra (iv). Finalmente, esta sub-rotina devolve a coleção de nós atualizada $\mathcal{F}_{\mathcal{B}}$.

\section{O algoritmo principal}

Apresentaremos agora o algoritmo principal.

Descrição de algoritmo. Poset-Forest-SEARCh recebe um conjunto finito e não-vazio $S$ e uma função custo $c$ decomponível em curvas em $\mathrm{U}$, e devolve uma coleção $\mathcal{M} \subseteq \mathcal{P}(S)$ que contém todos os elementos em $\mathcal{P}(S)$ de custo mínimo. 
Poset-Forest-Search $(S, c)$

$1 \mathcal{M} \leftarrow \emptyset$

$2 f$ é uma bijeção dos elementos de $S$ para $\{1, \ldots, n\}$

$3 \mathbf{T}[$ vertex $] \leftarrow \emptyset$

$4 \quad \mathbf{T}^{\prime}[$ vertex $] \leftarrow S$

$5 \quad \mathbf{T}[$ cost $] \leftarrow \mathbf{T}^{\prime}[\cos t] \leftarrow \infty$

$6 \mathbf{T}[$ adjacent $] \leftarrow \mathbf{T}^{\prime}[$ adjacent $] \leftarrow S$

$7 \quad \mathbf{T}[$ leftmost $] \leftarrow \mathbf{T}^{\prime}[$ leftmost $] \leftarrow 1$

$8 \mathcal{F}_{\mathcal{A}} \leftarrow\{\mathbf{T}\}$

$9 \quad \mathcal{F}_{\mathcal{B}} \leftarrow\left\{\mathbf{T}^{\prime}\right\}$

10 enquanto $\mathcal{F}_{\mathcal{A}} \neq \emptyset$

faça direction $\leftarrow$ SELECT-DIRECTION

se direction $=\mathrm{UP}$

então $\left\langle\mathcal{N}, \mathcal{F}_{\mathcal{A}}, \mathbf{N}\right\rangle \leftarrow$ Lower-Forest-Branch $\left(\mathcal{F}_{\mathcal{A}}, \mathcal{F}_{\mathcal{B}}, c, f\right)$

$\mathcal{F}_{\mathcal{B}} \leftarrow$ Upper-Forest-Pruning $\left(\mathcal{F}_{\mathcal{B}}, \mathbf{N}, f\right)$

senão $\left\langle\mathcal{N}, \mathcal{F}_{\mathcal{B}}, \mathbf{N}\right\rangle \leftarrow \operatorname{Upper}-$ Forest-Branch $\left(\mathcal{F}_{\mathcal{A}}, \mathcal{F}_{\mathcal{B}}, c, f\right)$

$\mathcal{F}_{\mathcal{A}} \leftarrow$ Lower-Forest-Pruning $\left(\mathcal{F}_{\mathcal{A}}, \mathbf{N}, f\right)$

$\mathcal{M} \leftarrow \mathcal{M} \cup \mathcal{N}$

17

18 devolva $\{M \in \mathcal{M}: c(M)$ é mínimo $\}$

A função Select-Direction devolve ou UP ou DOWN, de acordo com uma distribuição de probabilidade arbitrária.

A simulação mostrada a partir da figura 6.8 é equivalente a uma execução parcial de PFS com parâmetros $S=11111$ e $c$ definida pelos números ao lado dos nós.

Análise de complexidade de tempo. A inicialização das florestas, que é feita nas linhas 1-9, exige $O(n)$ unidades de tempo.

O laço enquanto nas linhas $17-17$ itera $k$ vezes, até que as coleções de nós $\mathcal{F}_{\mathcal{A}}$ e $\mathcal{F}_{\mathcal{B}}$ estejam vazias. Em cada iteração $i$ deste laço, $1 \leq i \leq k$, uma chamada de LowER-ForEST-BrAnch na linha 13 (no caso dual, UPPER-FOREST-BRANCH na linha 15) consome $O\left(n f(n)+n^{2}\left(\log \left|\mathcal{F}_{\mathcal{A}}\right|+\right.\right.$ $\left.\left.\log \left|\mathcal{F}_{\mathcal{B}}\right|\right)\right)$ unidades de tempo, enquanto que uma chamada de UpPer-Forest-Pruning na linha 14 (Lower-Forest-Pruning na linha 16) exige $O\left(n^{3} \log \left|\mathcal{F}_{\mathcal{B}}\right|\right)\left(O\left(n^{3} \log \left|\mathcal{F}_{\mathcal{A}}\right|\right)\right)$ unidades de tempo.

Portanto, Poset-Forest-Search requer $O\left(k n^{2} f(n)+k n^{3}\left(\log \left|\mathcal{F}_{\mathcal{A}}\right|+\log \left|\mathcal{F}_{\mathcal{B}}\right|\right)\right)$ unidades de tempo. Finalmente, seja $p$ o número total de vezes que a função custo $c$ é computada durante a execução do algoritmo. Como $p=O\left(k n^{2}\right)$, o consumo de tempo deste algoritmo é de

$$
\begin{aligned}
O\left(k n^{2} f(n)+k n^{3}\left(\log \left|\mathcal{F}_{\mathcal{A}}\right|+\log \left|\mathcal{F}_{\mathcal{B}}\right|\right)\right) & =O\left(p f(n)+p n\left(\log \left|\mathcal{F}_{\mathcal{A}}\right|+\log \left|\mathcal{F}_{\mathcal{B}}\right|\right)\right) \\
& =O\left(\left(f(n)+n\left(\log \left|\mathcal{F}_{\mathcal{A}}\right|+\log \left|\mathcal{F}_{\mathcal{B}}\right|\right)\right) p\right)
\end{aligned}
$$

unidades de tempo. Uma vez que uma execução deste algoritmo pode explorar uma fração do espaço de busca que é proporcional ao tamanho total do reticulado Booleano de grau $n$, o limite superior real para o tempo computacional assintótico de Poset-Forest-SEARCH é

$$
\begin{array}{rlrl}
O\left(\left(f(n)+n\left(\log \left|\mathcal{F}_{\mathcal{A}}\right|+\log \left|\mathcal{F}_{\mathcal{B}}\right|\right)\right) p\right) & =O\left(\left(f(n)+n\left(\log 2^{n}+\log 2^{n}\right)\right) 2^{n}\right) & \\
& =O\left(\left(f(n)+n\left(\log 2^{n}\right)\right) 2^{n}\right) & \\
& =O\left((f(n)+n(n)) 2^{n}\right) & & \text { (log base 2) } \\
& =O\left(\left(f(n)+n^{2}\right) 2^{n}\right) . &
\end{array}
$$




\subsection{Experimentos}

Nesta seção apresentaremos uma avaliação experimental dos algoritmos UBB e PFS. Esta avaliação foi feita realizando experimentos ótimos e sub-ótimos conforme a descrição fornecida na seção 2.2.2; tais experimentos utilizaram como função custo a equação 3.1, e como algoritmos UCS, SFFS e ES (i.e., a busca exaustiva descrita no apêndice B).

Na tabela 6.1 mostramos os resultados do experimento ótimo. Do ponto de vista semântico, SFFS teve um desempenho pobre neste experimento: primeiro, para o grupo das menores instâncias (7) ele encontrou uma melhor solução em apenas 51 de 100 instâncias; segundo, com o incremento do tamanho das instâncias, era menos provável que SFFS fornecesse uma solução ótima. ES, UBB e PFS são equivalentes, uma vez que todos eles sempre fornecem uma solução ótima. Do ponto de vista de desempenho computacional, para grupos de instâncias de tamanho 7 até 13, UBB e PFS computaram um número similar de vezes a função custo. Para instâncias de tamanho 13 e maiores, PFS computou menos vezes a função custo do que UBB: por exemplo, para 18 o algoritmo PFS computou a função custo em média 84104, 81 vezes, enquanto que o algoritmo UBB computou a mesma função custo em média 138287, 62 vezes, um acréscimo de mais de 64\%. Por outro lado, PFS gasta maior tempo computacional que SFFS e que UBB, embora PFS ganhe de ES para instâncias de tamanho 15 e maiores: este fenômeno ocorre devido ao maior custo computacional de controle de PFS ser compensado pelo menor número de nós computados em relação a ES.

Na tabela 6.2 mostramos os resultados da primeira e da segunda etapa do experimento subótimo, enquanto que na tabela 6.3 resumimos os resultados da terceira etapa desse experimento. PFS tem uma melhor semântica do que todos os demais algoritmos: por exemplo, para 18 o algoritmo PFS forneceu uma melhor solução dentre todos os algoritmos em $83 \%$ das instâncias, enquanto que o algoritmo UBB forneceu uma melhor solução em $50 \%$ das instâncias, ES em 38\%, e SFFS em apenas $12 \%$.

\subsubsection{Análise dos experimentos}

Apresentaremos a análise do uso de memória do algoritmo PFS, o qual é medido a partir dos resultados obtidos nos experimentos ótimos e sub-ótimos apresentados na seção anterior.

Para medirmos o uso de memória para cada experimento, executamos o algoritmo PFS em grupos de cem instâncias de mesmo tamanho (uma execução por instância). Para cada instância, armazenamos o tamanho máximo atingido pelas florestas durante a execução do algoritmo. Finalmente, para cada grupo de instâncias, calculamos a média do tamanho máximo de cada floresta para o grupo de instâncias de mesmo tamanho e fizemos a soma desses dois valores. Nas figuras 6.15(a) e 6.15(b) apresentamos gráficos desse cálculo em função do tamanho da instância, para, respectivamente, os experimentos ótimos e sub-ótimos. Os dados utilizados para desenhar estes gráficos são os mesmos apresentados nas tabelas 6.1 e 6.3. Em ambos os experimentos, os gráficos sugerem que o uso assintótico de memória é limitado superiormente por uma pequena proporção do tamanho do espaço de busca.

\subsection{Comentários}

Apresentamos neste capítulo dois algoritmos do tipo branch-and-bound para abordar o problema U-curve: U-Curve-Branch-And-Bound (UBB) e Poset-Forest-Search (PFS). PFS generaliza o algoritmo UBB, porém a um maior custo computacional em sua execução. Os resultados mostrados e discutidos na seção 6.3 sugerem que o algoritmo PFS apresenta melhor desempenho do que os algoritmos UBB, ES e SFFS.

Todavia, há espaço para melhoramentos nos algoritmos UBB e PFS: a escolha arbitrária de uma enumeração dos elementos do espaço de busca implica no percorrimento do mesmo como uma árvore ou floresta, o que provoca uma perda de parcela considerável da estrutura do reticulado Booleano que constitui o espaço de busca completo. Portanto, uma abordagem seria relaxar a enumeração 


\begin{tabular}{|c|c|c|c|c|c|c|c|c|c|}
\hline \multicolumn{2}{|c|}{ Instância } & \multicolumn{4}{|c|}{ Tempo (seg) } & \multicolumn{4}{|c|}{ \# Nós computados } \\
\hline$\overline{|S|}$ & $2^{|S|}$ & $\overline{\mathrm{ES}}$ & UBB & PFS & SFFS & ES & $\mathrm{UBB}$ & PFS & SFFS \\
\hline 7 & 128 & 0,19 & 0,21 & 0,26 & 0,23 & 128 & 89,17 & 83,84 & 138,88 \\
\hline 8 & 256 & 0,24 & 0,23 & 0,38 & 0,19 & 256 & 186,42 & 177,12 & 205,91 \\
\hline 9 & 512 & 0,35 & 0,31 & 0,35 & 0,20 & 512 & 328,36 & 331,64 & 252,84 \\
\hline 10 & 1024 & 0,42 & 0,26 & 0,39 & 0,18 & 1024 & 641,75 & 742,20 & 340,22 \\
\hline 11 & 2048 & 0,47 & 0,37 & 0,53 & 0,20 & 2048 & 1274,05 & 1204,32 & 500,69 \\
\hline 12 & 4096 & 0,65 & 0,40 & 0,79 & 0,21 & 4096 & 2263,40 & 2271,10 & 585,28 \\
\hline 13 & 8192 & 1,14 & 0,72 & 1,39 & 0,21 & 8192 & 4819,56 & 4162,96 & 653,75 \\
\hline 14 & 16384 & 2,06 & 1,28 & 2,30 & 0,22 & 16384 & 9956,72 & 7398,51 & 871,46 \\
\hline 15 & 32768 & 3,91 & 2,39 & 3,56 & 0,27 & 32768 & 19347,12 & 11055,91 & 1059,22 \\
\hline 16 & 65536 & 7,98 & 4,18 & 6,85 & 0,28 & 65536 & 35279,68 & 20789,05 & 1167,22 \\
\hline 17 & 131072 & 16,51 & 10,26 & 14,77 & 0,41 & 131072 & 85149,72 & 43066,65 & 1768,91 \\
\hline 18 & 262144 & 34,06 & 17,54 & 29,84 & 0,26 & 262144 & 138287,62 & 84104,81 & 1475,09 \\
\hline
\end{tabular}

\begin{tabular}{ccccccc}
\hline \multicolumn{3}{c}{ Instância } & & \multicolumn{4}{c}{ \# Melhor solução } \\
\cline { 5 - 7 }$|S|$ & $2^{|S|}$ & & ES & UBB & PFS & SFFS \\
\hline 7 & 128 & & 100 & 100 & 100 & 51 \\
8 & 256 & & 100 & 100 & 100 & 39 \\
9 & 512 & & 100 & 100 & 100 & 31 \\
10 & 1024 & & 100 & 100 & 100 & 32 \\
11 & 2048 & & 100 & 100 & 100 & 22 \\
12 & 4096 & & 100 & 100 & 100 & 20 \\
13 & 8192 & & 100 & 100 & 100 & 16 \\
14 & 16384 & & 100 & 100 & 100 & 7 \\
15 & 32768 & & 100 & 100 & 100 & 6 \\
16 & 65536 & & 100 & 100 & 100 & 6 \\
17 & 131072 & & 100 & 100 & 100 & 6 \\
18 & 262144 & & 100 & 100 & 100 & 8 \\
\hline
\end{tabular}

Tabela 6.1: comparação ótima entre UBB, PFS, SFFS e Exhaustive-SEARCh (ES), utilizando como função custo a equação 3.1 (i.e., uma redução polinomial de instâncias do problema da soma de subconjuntos, um problema $N P$-difícil).

adotada, permitindo assim que, durante as iterações desses algoritmos, sejam visitados elementos que não são adjacentes pela enumeração, mas que o são pelo reticulado Booleano. 


\begin{tabular}{|c|c|c|c|c|c|c|}
\hline \multicolumn{2}{|c|}{ Instância } & \multicolumn{4}{|c|}{ \# Nós computados } & \multirow[t]{2}{*}{ Limiar } \\
\hline$|S|$ & $2^{|S|}$ & $\mathrm{ES}$ & UBB & PFS & SFFS & \\
\hline 7 & 128 & 45,37 & 43,52 & 29,67 & 72,07 & 73 \\
\hline 8 & 256 & 74,34 & 66,48 & 63,91 & $\overline{89,51}$ & 90 \\
\hline 9 & 512 & 180,57 & 163,06 & 90,25 & $\overline{237,28}$ & 238 \\
\hline 10 & 1024 & 264,20 & 240,32 & 195,92 & $\overline{212,49}$ & 265 \\
\hline 11 & 2048 & $\overline{357,03}$ & 406,06 & 378,51 & 261,99 & 407 \\
\hline 12 & 4096 & 794,93 & $\overline{630,10}$ & 632,37 & 352,66 & 795 \\
\hline 13 & 8192 & $1 \overline{638,05}$ & 1441,30 & 1145,10 & 589,22 & 1639 \\
\hline 14 & 16384 & $\overline{2718,18}$ & 2418,27 & 985,60 & 726,00 & 2719 \\
\hline 15 & 32768 & $\overline{5088,71}$ & 5071,53 & 1758,73 & 884,75 & 5089 \\
\hline 16 & 65536 & $\overline{7991,27}$ & 9689,43 & 1677,30 & 1030,39 & 9690 \\
\hline 17 & 131072 & 15375,34 & 16963,19 & 1748,22 & 1285,74 & 16964 \\
\hline 18 & 262144 & 39408,05 & $\overline{40705,19}$ & 1799,44 & 1516,66 & 40706 \\
\hline
\end{tabular}

Tabela 6.2: pré-processamento que produz os limiares para a busca sub-ótima em instâncias simuladas.

\begin{tabular}{|c|c|c|c|c|c|c|c|c|}
\hline \multicolumn{2}{|c|}{ Instância } & \multicolumn{4}{|c|}{ Tempo (seg) } & \multicolumn{3}{|c|}{ \# Nós con } \\
\hline$\overline{|S|}$ & $2^{|S|}$ & ES & $\mathrm{UB}$ & PFS & SFFS & $\mathrm{ES}$ & & $\overline{\mathrm{BB}}$ \\
\hline 7 & 128 & 0,12 & 0,1 & 0,12 & 0,14 & 73 & & 69 \\
\hline 8 & 256 & 0,14 & 0,1 & 0,16 & 0,11 & 90 & & 40 \\
\hline 9 & 512 & 0,19 & 0,1 & 0,25 & 0,10 & 238 & & 1,31 \\
\hline 10 & 1024 & 0,19 & 0,1 & 0,25 & 0,11 & 265 & & 9,99 \\
\hline 11 & 2048 & 0,20 & 0,20 & 0,33 & 0,13 & 407 & & 0,69 \\
\hline 12 & 4096 & 0,28 & 0,2 & 0,34 & 0,14 & 795 & & 5,85 \\
\hline 13 & 8192 & 0,32 & 0,27 & 0,56 & 0,15 & 1639 & & 74,80 \\
\hline 14 & 16384 & 0,40 & 0,4 & 0,94 & 0,19 & 2719 & & 71,09 \\
\hline 15 & 32768 & 0,83 & 0,68 & 1,57 & 0,22 & 5089 & & 31,27 \\
\hline 16 & 65536 & 1,36 & 1,1 & 2,76 & 0,21 & 9690 & & 3,17 \\
\hline 17 & 131072 & 2,33 & 1,9 & 4,68 & 0,29 & 16964 & & 37,76 \\
\hline \multirow[t]{15}{*}{18} & 262144 & 5,83 & $4,1 \xi$ & 9,54 & 0,33 & 40706 & 30 & 28,88 \\
\hline & & \multicolumn{3}{|c|}{ Instância } & \multicolumn{4}{|c|}{ \# Melhor solução } \\
\hline & & & $S \mid$ & $2^{|S|}$ & $\mathrm{ES}$ & UBB & PFS & SFFS \\
\hline & & & 7 & 128 & 65 & 70 & 70 & 30 \\
\hline & & & 8 & 256 & 53 & 50 & 58 & 24 \\
\hline & & & 9 & 512 & 46 & 52 & 71 & 16 \\
\hline & & & 10 & 024 & 38 & 56 & 68 & 20 \\
\hline & & & 11 & 048 & 34 & 54 & 57 & 16 \\
\hline & & & 12 & 096 & 34 & 57 & 63 & 16 \\
\hline & & & 13 & 192 & 46 & 46 & 56 & 16 \\
\hline & & & 14 & 384 & 34 & 49 & 60 & 9 \\
\hline & & & 15 & 768 & 33 & 50 & 62 & 13 \\
\hline & & & 16 & 536 & 30 & 42 & 81 & 9 \\
\hline & & & 17 & 1072 & 31 & 41 & 80 & 3 \\
\hline & & & 18 & 2144 & 38 & 50 & 83 & 12 \\
\hline
\end{tabular}

Tabela 6.3: busca sub-ótima em instâncias simuladas; os limiares para cada teste foram obtidos através do pré-processamento exibido na tabela 6.2. 


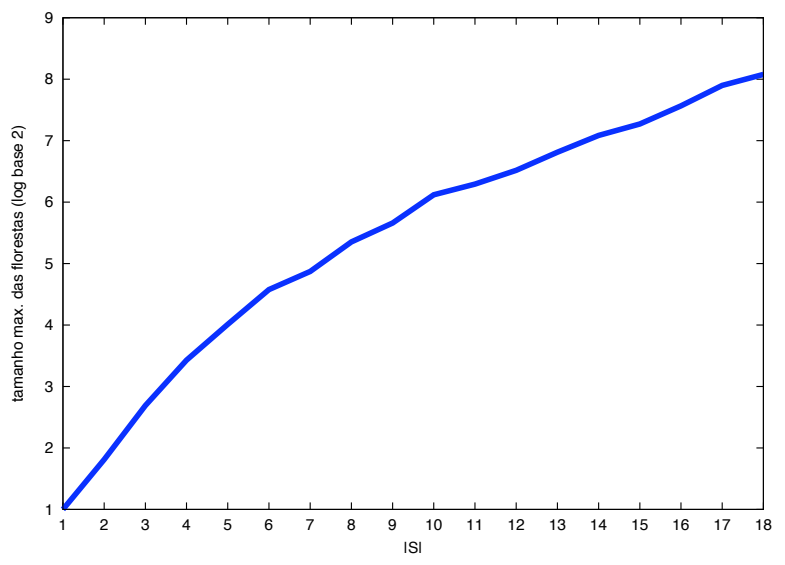

(a)

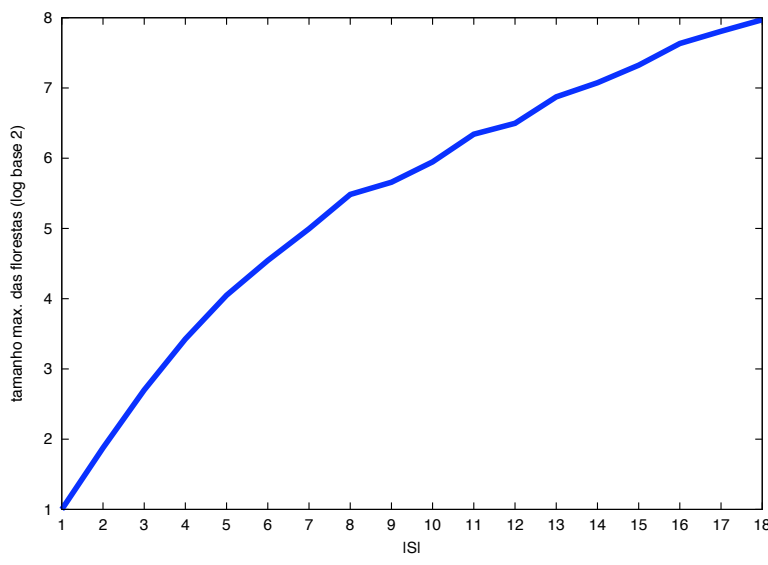

(b)

Figura 6.15: a evolução da soma dos tamanhos máximos das florestas $\mathcal{A}$ e $\mathcal{B}$ em uma busca ótima (figura 6.15(a)) e sub-ótima (figura 6.15(b)), durante execuções do algoritmo PFS em instâncias simuladas. Os gráficos sugerem que o tamanho das florestas tem um limite superior assintótico linear ao tamanho do espaço de busca. 


\section{Capítulo 7}

\section{Experimentos}

Neste capítulo, apresentaremos experimentos realizados com alguns dos algoritmos introduzidos neste trabalho, mais precisamente com U-CURVE-SEARCH (UCS), U-CuRVE-BRANCH-AND-Bound (UBB) e Poset-Forest-Search (PFS). Realizamos experimentos ótimos e sub-ótimos, envolvendo tanto instâncias simuladas a partir da redução polinomial da equação 3.1 quanto dados obtidos de um problema real, o qual surge na etapa de seleção de características em projeto de W-operadores.

Iniciaremos este capítulo apresentando as instâncias empregadas nos experimentos. Em seguida, mostraremos, para cada tipo de instância, os resultados obtidos nos experimentos ótimos e subótimos. Faremos uma análise dos resultados dos experimentos, incluindo uma avaliação do desempenho dos algoritmos. Finalmente, mostraremos uma verificação da consistência entre a análise teórica dos algoritmos e os resultados experimentais obtidos neste capítulo.

\subsection{Instâncias empregadas}

\subsubsection{Simulação de instâncias difíceis}

Neste experimento, as instâncias foram produzidas conforme descrevemos na seção 2.2.2. A função custo empregada pelos algoritmos foi a equação 3.1, isto é, uma redução polinomial de instâncias do problema da soma de subconjuntos, um problema NP-difícil, para instâncias do problema U-curve.

\subsubsection{Projeto de W-operadores}

Um W-operador é uma transformação de imagem que é localmente definida dentro de uma janela $W$ (i.e., um subconjunto do plano dos inteiros) e invariante por translação [BTHJH00]. Uma transformação de imagem é caracterizada por um classificador. Um passo no projeto de um classificador é a seleção de características (i.e., a escolha de um subconjunto de $W$ que otimiza algum critério de qualidade). A minimização da entropia condicional média é um critério de qualidade que mostrou eficácia no projeto de W-operadores [MJCJB06].

Sejam $X$ um subconjunto de uma janela $W$ e $\mathbf{X}$ uma variável aleatória em $\mathcal{P}(X)$. A entropia condicional de uma variável aleatória binária $Y$ dado $\mathbf{X}=\mathbf{x}$ é dada pela equação:

$$
H(Y \mid \mathbf{X}=\mathbf{x})=-\sum_{y \in Y} P(Y=y \mid \mathbf{X}=\mathbf{x}) \log P(Y=y \mid \mathbf{X}=\mathbf{x}),
$$

na qual $P($.) é a função de distribuição de probabilidade. Definiremos $\log 0$ como 0 . A entropia condicional média de $Y$ dado $\mathbf{X}$ é expressa por:

$$
E[H(Y \mid \mathbf{X})]=\sum_{\mathbf{x} \in \mathbf{X}} H(Y \mid \mathbf{X}=\mathbf{x}) P(\mathbf{X}=\mathbf{x}) .
$$


Na prática, os valores das distribuições condicionais de probabilidade são estimadas, portanto um alto erro de estimação pode ser induzido pela falta de amostras suficientes (i.e., pares $\langle y, \mathbf{x}\rangle$ raramente observados podem estar sub-representados). Para contornar o número insuficiente de amostras, é adotada a penalização para pares de valores que têm uma única observação: eles considerados como seguindo uma distribuição uniforme, portanto levando à entropia máxima. Dessa forma, adotando-se a penalização, a estimação da entropia condicional média é dada pela equação:

$$
\hat{E}[H(Y \mid \mathbf{X})]=\frac{N}{t}+\sum_{\mathbf{x} \in \mathbf{X}: \hat{P}(\mathbf{x})>\frac{1}{t}} \hat{H}(Y \mid \mathbf{X}=\mathbf{x}) \hat{P}(\mathbf{X}=\mathbf{x}),
$$

na qual $N$ é o número de valores de $\mathbf{X}$ com uma única ocorrência nas amostras e $t$ é o número total de amostras.

A entropia condicional média com penalização foi utilizada neste experimento como função custo. As amostras de treinamento foram obtidas de quatorze pares de imagens binárias apresentadas em Martins-Jr e colegas [MJCJB06], percorrendo uma janela $4 \times 4$, de tamanho 16, sobre cada imagem observada e obtendo assim o valor correspondente da imagem transformada. Os resultados foram quatorze conjuntos, cada um deles contendo 576 amostras. Para cada conjunto, executamos os algoritmos UCS, UBB, PFS e SFFS; para cada um destes algoritmos, armazenamos o tempo necessário para a execução, o número de nós computados (i.e., o número de vezes que a função custo foi computada) e verificamos se foi obtida uma melhor solução.

\subsection{Experimentos ótimos}

\subsubsection{Simulação de instâncias difíceis}

Na tabela 7.1 mostramos os resultados do experimento ótimo com instâncias difíceis. Do ponto de vista semântico, SFFS teve um desempenho pobre neste experimento: primeiro, somente para grupos de instâncias menores que 7 é que ele encontrou uma melhor solução em mais de $50 \%$ dos casos; segundo, com o incremento do tamanho das instâncias, era cada vez menos provável que SFFS fornecesse uma solução ótima. UCS, UBB e PFS são equivalentes, uma vez que todos eles fornecem uma solução ótima para o problema U-curve.

Do ponto de vista de desempenho computacional, para grupos de instâncias de tamanho até 11, UCS computou menos vezes a função custo do que SFFS. Além disso, UCS sempre computou menos vezes a função custo do que UBB e PFS: por exemplo, para grupo de instâncias de tamanho 18, UCS computou menos de $16 \%$ do que foi computado por UBB e menos de $28 \%$ do que foi computado por PFS (figura 7.1(b)). Em compensação, para grupos de instâncias a partir de 11, UCS sempre exigiu maior tempo computacional do que os demais algoritmos. Na comparação entre PFS e UBB, o primeiro computou menos vezes a função custo do que o segundo: por exemplo, para grupos de instâncias a partir de 11, PFS computou menos de $57 \%$ do que foi computado por UBB. Já na comparação de consumo de tempo entre UBB e PFS, para grupo de instâncias a partir de 8, UBB sempre exigiu menor tempo computacional do que PFS, embora a diferença de tempo seja bem menos significativa do que a verificada entre esses dois algoritmos e o UCS (figura 7.1(a)).

\subsubsection{Projeto de W-operadores}

Na tabela 7.2 resumimos os resultados do experimento ótimo em projeto de W-operadores, no qual cada arquivo foi utilizado uma vez para o procedimento de seleção de características. Em menos de $29 \%$ dos experimentos SFFS forneceu uma solução ótima. Por outro lado, UCS, UBB e PFS sempre forneceram uma solução ótima.

Do ponto de vista de desempenho computacional, UCS sempre computou menos vezes a função custo do que UBB e PFS. Além disso, embora UCS tenha continuado a ser o algoritmo que exigiu maior consumo de tempo, a diferença do mesmo em relação os demais algoritmos diminuiu sensivelmente em relação ao experimento com instâncias difíceis: enquanto que no experimento de 


\begin{tabular}{|c|c|c|c|c|c|c|c|c|c|}
\hline \multicolumn{2}{|c|}{ Instância } & \multicolumn{4}{|c|}{ Tempo (seg) } & \multicolumn{4}{|c|}{ \# Nós computados } \\
\hline$|S|$ & $2^{|S|}$ & $\mathrm{UCS}$ & UBB & PFS & SFFS & $\mathrm{UCS}$ & $\mathrm{UBB}$ & PFS & SFFS \\
\hline 5 & 32 & 0,08 & 0,08 & 0,06 & 0,07 & 21,37 & 25,16 & 25,86 & 44,81 \\
\hline 6 & 64 & 0,11 & 0,11 & 0,10 & 0,12 & 36,11 & 49,58 & 49,22 & 83,37 \\
\hline 7 & 128 & 0,11 & 0,10 & 0,10 & 0,09 & 57,89 & 82,65 & 96,87 & 88,90 \\
\hline 8 & 256 & 0,20 & 0,12 & 0,18 & 0,11 & 99,63 & 172,15 & 169,63 & 163,63 \\
\hline 9 & 512 & 0,22 & 0,21 & 0,24 & 0,19 & 173,77 & 364,53 & 369,81 & 323,80 \\
\hline 10 & 1024 & 0,28 & 0,20 & 0,23 & 0,14 & 266,64 & 712,60 & 647,94 & 443,08 \\
\hline 11 & 2048 & 0,47 & 0,28 & 0,43 & 0,12 & 485,25 & 1313,72 & 1150,59 & 408,18 \\
\hline 12 & 4096 & 0,94 & 0,41 & 0,67 & 0,21 & 788,32 & 2434,58 & 2261,01 & 563,78 \\
\hline 13 & 8192 & 2,29 & 0,71 & 1,48 & 0,25 & 1 121,39 & 5037,72 & 3913,47 & 790,04 \\
\hline 14 & 16384 & 4,27 & 1,35 & 2,26 & 0,33 & 2525,01 & 10213,42 & 7517,61 & 950,97 \\
\hline 15 & 32768 & 10,32 & 2,16 & 3,36 & 0,15 & 4215,71 & 18771,15 & 12623,43 & 1009,16 \\
\hline 16 & 65536 & 27,71 & 4,61 & 7,33 & 0,25 & 7664,27 & 38937,86 & 26403,44 & 1103,47 \\
\hline 17 & 131072 & 74,67 & 9,40 & 12,08 & 0,31 & 11467,97 & 79970,50 & 41279,85 & 1619,06 \\
\hline 18 & 262144 & 315,21 & 16,58 & 23,44 & 0,41 & 21991,57 & 141241,26 & 79790,37 & 1453,98 \\
\hline
\end{tabular}

\begin{tabular}{|c|c|c|c|c|c|}
\hline \multicolumn{2}{|c|}{ Instância } & \multicolumn{4}{|c|}{ \# Melhor solução } \\
\hline$|S|$ & $2^{|S|}$ & UCS & UBB & PFS & SFFS \\
\hline 5 & 32 & 100 & 100 & 100 & 77 \\
\hline 6 & 64 & 100 & 100 & 100 & 53 \\
\hline 7 & 128 & 100 & 100 & 100 & 39 \\
\hline 8 & 256 & 100 & 100 & 100 & 25 \\
\hline 9 & 512 & 100 & 100 & 100 & 31 \\
\hline 10 & 1024 & 100 & 100 & 100 & 26 \\
\hline 11 & 2048 & 100 & 100 & 100 & 14 \\
\hline 12 & 4096 & 100 & 100 & 100 & 13 \\
\hline 13 & 8192 & 100 & 100 & 100 & 8 \\
\hline 14 & 16384 & 100 & 100 & 100 & 9 \\
\hline 15 & 32768 & 100 & 100 & 100 & 13 \\
\hline 16 & 65536 & 100 & 100 & 100 & 7 \\
\hline 17 & 131072 & 100 & 100 & 100 & 7 \\
\hline 18 & 262144 & 100 & 100 & 100 & 5 \\
\hline
\end{tabular}

Tabela 7.1: comparação entre UCS, UBB, PFS e SFFS, utilizando como função custo a equação 3.1 (i.e., uma redução polinomial de instâncias do problema da soma de subconjuntos, um problema NP-difícil).

tamanho 18 da tabela 7.1 UCS exigiu 19 vezes mais tempo que o UBB e 14 vezes mais tempo que PFS, no experimento no projeto de W-operadores, em média, a proporção foi de 2,5 vezes e de 1, 4 vezes, respectivamente (figura 7.2(a)). Tal diferença explica-se por dois fatos:

- em média, UCS computou mais nós nos experimentos de W-operador do que nos experimentos com instâncias difíceis de mesmo tamanho (16);

- a função custo da entropia condicional média penalizada é bem mais cara computacionalmente do que a função custo da redução polinomial do problema da soma de subconjuntos.

Por fim, na comparação entre UBB e PFS, vemos que, em média, UBB computou menos vezes a função custo, ocorrendo exatamente o oposto do que foi verificado no experimento com instâncias difíceis de tamanho 16. Este fato pode ser explicado pela maioria dos mínimos das cadeias das instâncias nos experimentos de W-operador estarem localizados na metade inferior da cintura do reticulado Booleano, o que favorece a busca de baixo para cima do UBB (figura 7.2(b)). 


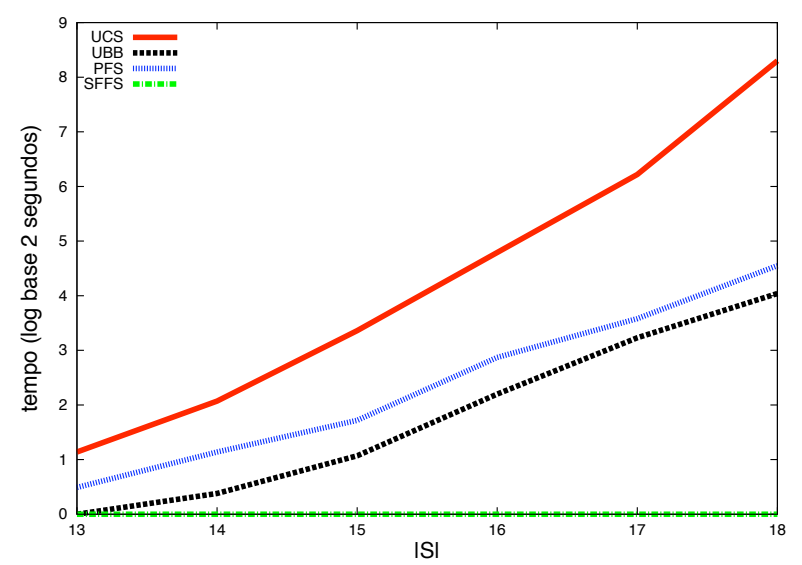

(a)

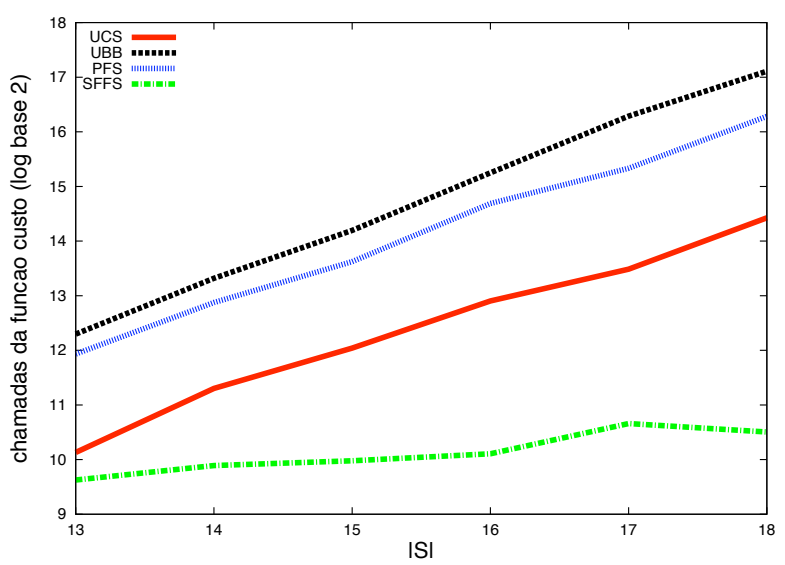

(b)

Figura 7.1: comparação entre UCS, UBB, PFS e SFFS, no experimento ótimo com instâncias dificeis simuladas. Na figura 7.1(a) é mostrada uma comparação de tempo médio que os algoritmos gastaram para resolver diversos tamanhos de instâncias; já na figura \%.1(b) é exibido o número médio de nós computados pelos algoritmos para os mesmos tamanhos de instâncias.

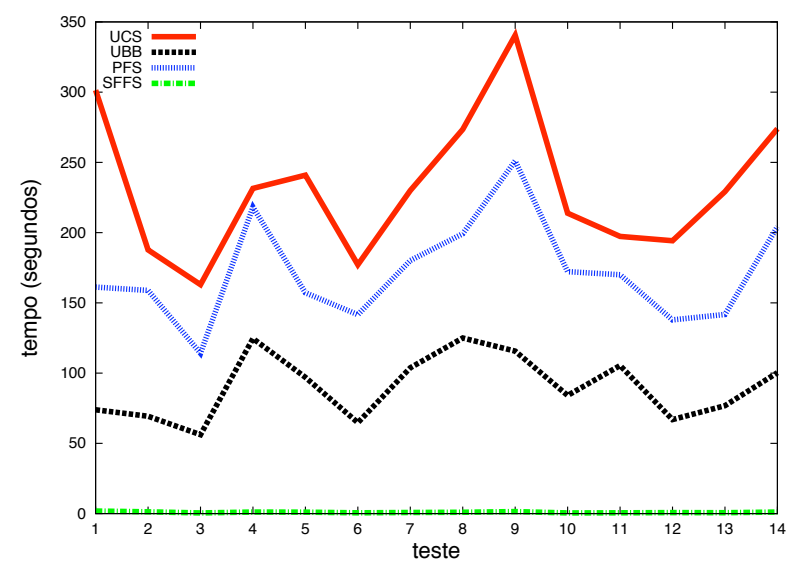

(a)

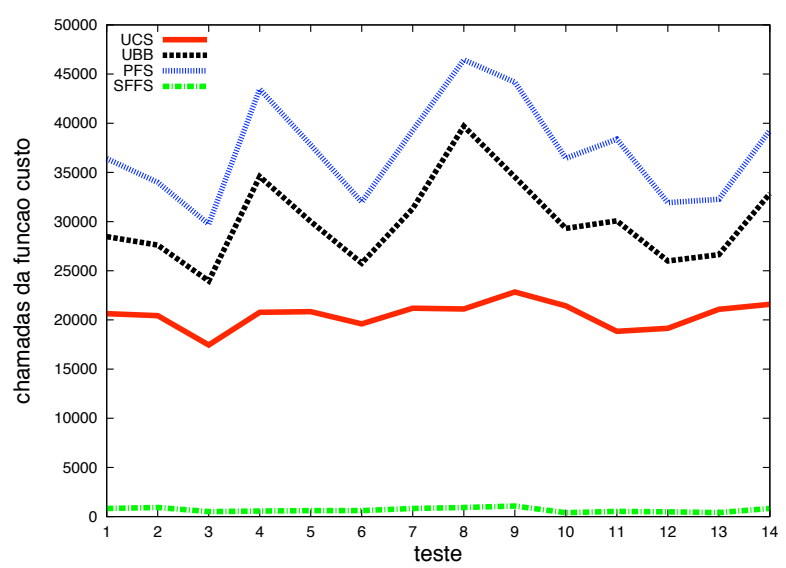

(b)

Figura 7.2: comparação entre UCS, UBB, PFS e SFFS, no experimento ótimo de projeto de $W$-operador. Na figura 7.2(a) é mostrada uma comparação de tempo médio que os algoritmos gastaram para resolver cada teste; já na figura 7.2(b) é exibido o número médio de nós computados pelos algoritmos para os mesmos testes. 


\begin{tabular}{|c|c|c|c|c|c|c|c|c|c|}
\hline \multirow[t]{2}{*}{ \# Teste } & \multicolumn{4}{|c|}{ Tempo (seg) } & \multicolumn{5}{|c|}{ \# Nós computados } \\
\hline & $\mathrm{UCS}$ & $\mathrm{UBB}$ & $\mathrm{PFS}$ & SFFS & \multicolumn{2}{|c|}{$\overline{\mathrm{UCS}}$} & $\mathrm{UBB}$ & PFS & SFFS \\
\hline 1 & 301,39 & 73,91 & 161,20 & 1,84 & \multicolumn{2}{|c|}{20636} & 28468 & 36403 & 827 \\
\hline 2 & 187,77 & 69,30 & 158,86 & 1,29 & \multicolumn{2}{|c|}{20433} & 27610 & 33989 & 931 \\
\hline 3 & 162,96 & 56,17 & 114,17 & 0,51 & \multicolumn{2}{|c|}{17447} & 23960 & 29743 & 507 \\
\hline 4 & 231,48 & 124,61 & 218,11 & 1,11 & \multicolumn{2}{|c|}{20775} & 34574 & 43467 & 564 \\
\hline 5 & 240,93 & 97,03 & 157,23 & 1,01 & \multicolumn{2}{|c|}{20845} & 30009 & 37792 & 615 \\
\hline 6 & 177,14 & 64,73 & 141,62 & 0,59 & \multicolumn{2}{|c|}{19600} & 25773 & 31982 & 615 \\
\hline 7 & 229,98 & 103,77 & 180,16 & 0,81 & \multicolumn{2}{|c|}{21187} & 31319 & 39262 & 827 \\
\hline 8 & 273,53 & 124,97 & 199,18 & 0,96 & \multicolumn{2}{|c|}{21112} & 39691 & 46456 & 929 \\
\hline 9 & 340,51 & 115,66 & 251,05 & 1,40 & \multicolumn{2}{|c|}{22846} & 34532 & 44179 & 1070 \\
\hline 10 & 213,86 & 84,10 & 172,21 & 0,46 & \multicolumn{2}{|c|}{21424} & 29310 & 36438 & 401 \\
\hline 11 & 197,30 & 105,38 & 170,02 & 0,57 & \multicolumn{2}{|c|}{18839} & 30080 & 38379 & 534 \\
\hline 12 & 194,26 & 66,94 & 137,87 & 0,71 & \multicolumn{2}{|c|}{19150} & 25998 & 31932 & 483 \\
\hline 13 & 229,23 & 76,79 & 141,78 & 0,61 & \multicolumn{2}{|c|}{21078} & 26636 & 32264 & 403 \\
\hline 14 & 273,99 & 100,32 & 204,20 & 1,09 & \multicolumn{2}{|c|}{21587} & 32849 & 39272 & 827 \\
\hline \multirow[t]{18}{*}{ Média } & 232,45 & 90,26 & 171,98 & 0,93 & 204 & 7,07 & 30057,79 & 37254,14 & $\overline{680,93}$ \\
\hline & & \multirow{2}{*}{\multicolumn{2}{|c|}{ \# Teste }} & \multicolumn{4}{|c|}{ \# Melhor solução } & & \\
\hline & & & & $\mathrm{UCS}$ & $\mathrm{UBB}$ & $\mathrm{PFs}$ & SFFS & & \\
\hline & & 1 & & 1 & 1 & 1 & 1 & & \\
\hline & & 2 & & 1 & 1 & 1 & 0 & & \\
\hline & & 3 & & 1 & 1 & 1 & 0 & & \\
\hline & & 4 & & 1 & 1 & 1 & 1 & & \\
\hline & & 5 & & 1 & 1 & 1 & 0 & & \\
\hline & & 6 & & 1 & 1 & 1 & 1 & & \\
\hline & & 7 & & 1 & 1 & 1 & 0 & & \\
\hline & & 8 & & 1 & 1 & 1 & 0 & & \\
\hline & & 9 & & 1 & 1 & 1 & 0 & & \\
\hline & & 10 & & 1 & 1 & 1 & 0 & & \\
\hline & & 1. & & 1 & 1 & 1 & 1 & & \\
\hline & & $1:$ & & 1 & 1 & 1 & 0 & & \\
\hline & & $1:$ & & 1 & 1 & 1 & 0 & & \\
\hline & & 1 & & 1 & 1 & 1 & 0 & & \\
\hline & & Mé & & 1 & 1 & 1 & 0,29 & & \\
\hline
\end{tabular}

Tabela 7.2: comparação entre UCS, UBB, PFS e SFFS, no projeto de $W$-operador, utilizando como função custo a entropia condicional média penalizada (equação \%.2).

\subsection{Experimentos sub-ótimos}

\subsubsection{Simulação de instâncias difíceis}

Na tabela 7.3 mostramos os resultados da primeira e da segunda etapa do experimento subótimo, enquanto que na tabela 7.4 resumimos os resultados da terceira etapa. UCS tem uma melhor semântica do que todos os demais algoritmos: em experimentos de tamanho 18, ele encontrou uma melhor resposta em 97\% dos casos. PFS teve a segunda melhor semântica, encontrando uma melhor resposta em $74 \%$ dos casos. UBB encontrou uma melhor resposta em $34 \%$ dos casos, e SFFS em apenas $8 \%$ (figura $7.3(\mathrm{c})$ ).

Do ponto de vista de desempenho computacional, UCS sempre computou menos vezes a função custo do que UBB e PFS: por exemplo, para instâncias de tamanho 18, UCS computou a função custo 2 vezes menos que UBB e 1,5 vezes menos que PFS (figura 7.3(b)). Todavia, UCS consumiu 


\begin{tabular}{|c|c|c|c|c|c|c|}
\hline \multicolumn{2}{|c|}{ \# Teste } & \multicolumn{4}{|c|}{ \# Nós computados } & \multirow[t]{2}{*}{ Limiar } \\
\hline$\overline{|S|}$ & $2^{|S|}$ & UCS & UBB & PFS & SFFS & \\
\hline 5 & 32 & 11,35 & 12,83 & 11,05 & 20,53 & 21 \\
\hline 6 & 64 & 17,52 & 20,61 & 19,99 & $\overline{33,21}$ & 34 \\
\hline 7 & 128 & 27,03 & 48,64 & 33,24 & 70,32 & 71 \\
\hline 8 & 256 & 34,64 & 60,34 & 58,39 & $\overline{101,19}$ & 102 \\
\hline 9 & 512 & 49,13 & 166,38 & 127,78 & $\overline{237,03}$ & 238 \\
\hline 10 & 1024 & 68,83 & 223,78 & 177,47 & $\overline{216,05}$ & 224 \\
\hline 11 & 2048 & 70,88 & $\overline{424,89}$ & 266,03 & 341,89 & 425 \\
\hline 12 & 4096 & 112,87 & $\overline{877,64}$ & 665,14 & 529,85 & 878 \\
\hline 13 & 8192 & 118,01 & $1 \overline{651,59}$ & 1000,35 & 670,45 & 1652 \\
\hline 14 & 16384 & 197,32 & $\overline{2000,01}$ & 804,19 & 513,45 & 2001 \\
\hline 15 & 32768 & 206,33 & $\overline{6142,86}$ & 1118,60 & 1003,60 & 6143 \\
\hline 16 & 65536 & 262,97 & $\overline{6427,58}$ & 1236,76 & 886,75 & 6428 \\
\hline 17 & 131072 & 234,68 & $\overline{15654,89}$ & 1654,95 & 1208,01 & 15655 \\
\hline 18 & 262144 & 505,56 & 44548,57 & 2519,01 & 1760,32 & 44549 \\
\hline
\end{tabular}

Tabela 7.3: pré-processamento que produz os limiares para a busca sub-ótima em instâncias difíceis.

muito mais tempo para executar, 24 vezes mais que o UBB e 12 vezes mais do que o PFS para instâncias de tamanho 18. Na comparação entre UBB e PFS, o primeiro computou em média mais vezes a função custo e consumiu menos tempo do que o segundo (figura 7.3(a)).

\subsubsection{Projeto de W-operadores}

Na tabela 7.5 mostramos os resultados da primeira e da segunda etapa do experimento subótimo, enquanto que na tabela 7.6 resumimos os resultados da terceira etapa. UCS teve o melhor desempenho semântico, encontrando uma melhor solução em $93 \%$ dos experimentos. UBB foi o segundo melhor algoritmo, encontrando uma melhor solução em $64 \%$ dos casos. PFS e SFFS encontraram uma melhor solução em $50 \%$ e $29 \%$ dos casos, respectivamente.

Do ponto de vista de desempenho computacional, UCS sempre computou menos vezes a função custo do que UBB e PFS: em média, UCS computou a função custo $30 \%$ menos que UBB e $45 \%$ menos que PFS (figura 7.4(b)). Todavia, UCS consumiu mais tempo para executar, em média $120 \%$ a mais que o UBB e $30 \%$ a mais do que o PFS. Na comparação entre UBB e PFS, o primeiro computou em média mais vezes a função custo e consumiu menos tempo do que o segundo (figura 7.4(a)). Além disso, embora UCS tenha continuado a ser o algoritmo que exigiu maior consumo de tempo, a diferença do mesmo em relação os demais algoritmos diminuiu sensivelmente em relação ao experimento sub-ótimo com instâncias difíceis, pela mesmas razões já apontadas nos experimentos ótimos de projeto de $\mathrm{W}$-operador (seção 7.2.2).

\subsection{Análise dos resultados}

Nesta seção, discutiremos os resultados teóricos e experimentais apresentados neste trabalho. Inicialmente analisaremos alguns aspectos da performance dos algoritmos. Em seguida, estudaremos a consistência entre o tempo computacional assintótico dos algoritmos e os resultados experimentais obtidos nesta seção.

\subsubsection{Análise de desempenho dos algoritmos}

Para realizarmos a comparação de performance entre os algoritmos UCS, UBB e PFS nos experimentos difíceis, nós executamos estes algoritmos em grupos compostos por cem diferentes 


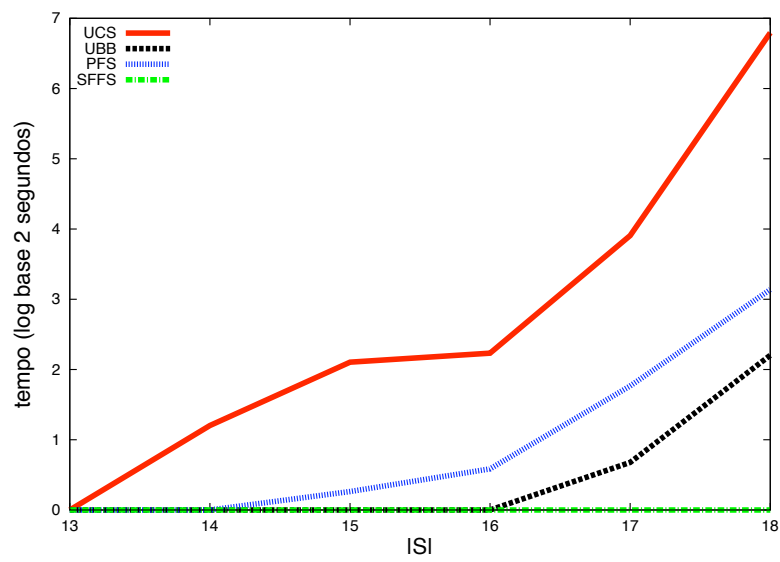

(a)

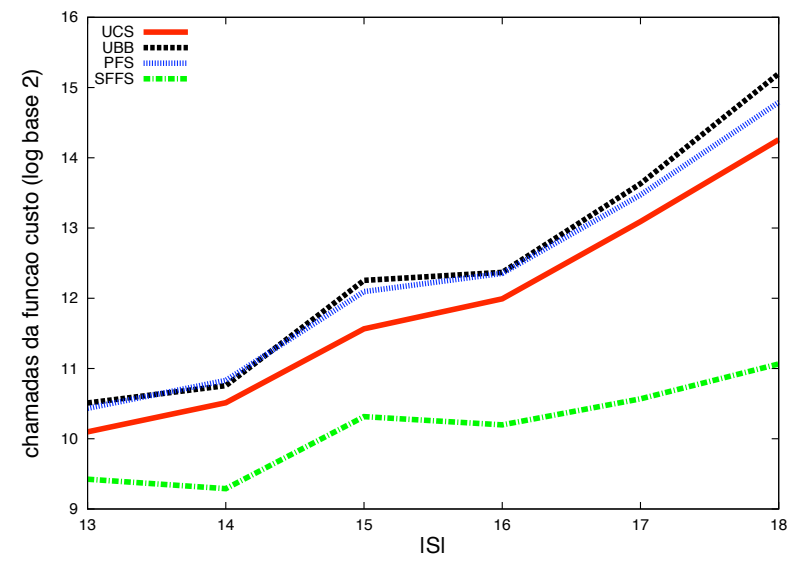

(b)

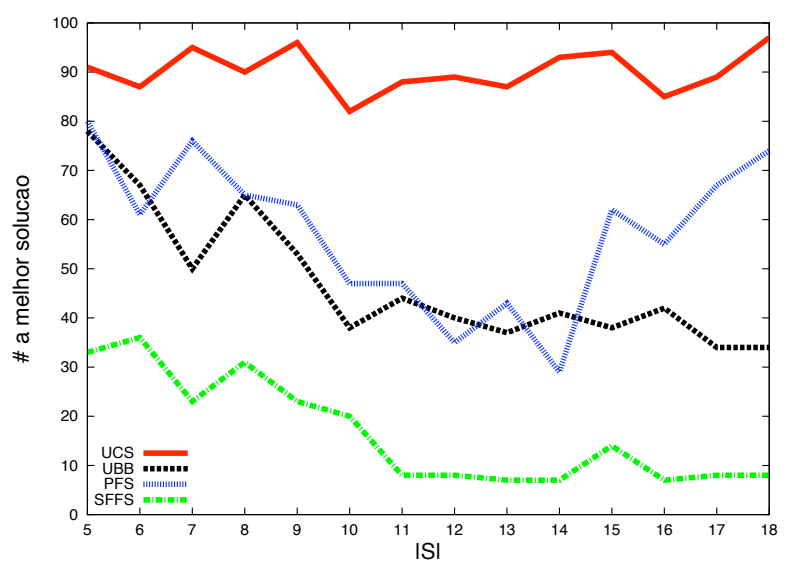

(c)

Figura 7.3: comparação entre UCS, UBB, PFS e SFFS, no experimento sub-ótimo com instâncias difíceis simuladas. Na figura 7.3(a) é mostrada uma comparação de tempo médio que os algoritmos gastaram para resolver diversos tamanhos de instâncias; já na figura 7.3(b) é exibido o número médio de nós computados pelos algoritmos para os mesmos tamanhos de instâncias; por fim, na figura 7.3(c) é fornecido o número de vezes que cada algoritmo encontrou uma melhor solução, também para os mesmos tamanhos de instâncias. 


\begin{tabular}{|c|c|c|c|c|c|c|c|c|c|}
\hline \multicolumn{2}{|c|}{ Instância } & \multicolumn{4}{|c|}{ Tempo (seg) } & \multicolumn{4}{|c|}{ \# Nós computados } \\
\hline$|S|$ & $2^{|S|}$ & UCS & UBB & PFS & SFFS & $\mathrm{UCS}$ & UBB & PFS & $\overline{\text { SFFS }}$ \\
\hline 5 & 32 & 0,01 & 0,01 & 0,01 & 0,01 & 19,01 & 19,42 & 19,57 & 19,37 \\
\hline 6 & 64 & 0,01 & 0,01 & 0,01 & 0,01 & 31,15 & 30,09 & 30,81 & 27,70 \\
\hline 7 & 128 & 0,03 & 0,02 & 0,02 & 0,01 & 53,62 & 63,76 & 58,27 & 51,80 \\
\hline 8 & 256 & 0,06 & 0,02 & 0,02 & 0,01 & 77,21 & 80,09 & 84,48 & 57,30 \\
\hline 9 & 512 & 0,08 & 0,02 & 0,05 & 0,02 & 139,02 & 187,49 & 182,24 & 127,82 \\
\hline 10 & 1024 & 0,11 & 0,03 & 0,05 & 0,03 & 179,07 & 195,72 & 190,00 & 128,72 \\
\hline 11 & 2048 & 0,21 & 0,06 & 0,10 & 0,03 & 303,18 & 369,09 & 364,32 & 228,77 \\
\hline 12 & 4096 & 0,44 & 0,10 & 0,20 & 0,04 & 569,51 & 750,98 & 742,54 & 429,80 \\
\hline 13 & 8192 & 0,91 & 0,19 & 0,42 & 0,07 & 1096,20 & 1458,08 & 1382,60 & 687,77 \\
\hline 14 & 16384 & 1,36 & 0,26 & 0,52 & 0,09 & 1461,51 & 1730,44 & 1822,33 & 626,48 \\
\hline 15 & 32768 & 4,39 & 0,62 & 1,23 & 0,16 & 3030,70 & 4885,28 & 4375,61 & 1274,03 \\
\hline 16 & 65536 & 4,71 & 0,68 & 1,54 & 0,12 & 4073,06 & 5285,58 & 5237,42 & 1134,41 \\
\hline 17 & 131072 & 15,31 & 1,60 & 3,48 & 0,16 & 8731,30 & 12698,82 & 11355,93 & 1518,89 \\
\hline 18 & 262144 & 111,48 & 4,66 & 8,87 & 0,20 & 19587,91 & 37554,74 & 28310,49 & 2143,14 \\
\hline
\end{tabular}

\begin{tabular}{|c|c|c|c|c|c|}
\hline \multicolumn{2}{|c|}{ Instância } & \multicolumn{4}{|c|}{ \# Melhor solução } \\
\hline$\overline{|S|}$ & $2^{|S|}$ & UCS & UBB & PFS & SFFS \\
\hline 5 & 32 & 91 & 78 & 80 & 33 \\
\hline 6 & 64 & 87 & 67 & 61 & 36 \\
\hline 7 & 128 & 95 & 50 & 76 & 23 \\
\hline 8 & 256 & 90 & 65 & 65 & 31 \\
\hline 9 & 512 & 96 & 53 & 63 & 23 \\
\hline 10 & 1024 & 82 & 38 & 47 & 20 \\
\hline 11 & 2048 & 88 & 44 & 47 & 8 \\
\hline 12 & 4096 & 89 & 40 & 35 & 8 \\
\hline 13 & 8192 & 87 & 37 & 43 & 7 \\
\hline 14 & 16384 & 93 & 41 & 29 & 7 \\
\hline 15 & 32768 & 94 & 38 & 62 & 14 \\
\hline 16 & 65536 & 85 & 42 & 55 & 7 \\
\hline 17 & 131072 & 89 & 34 & 67 & 8 \\
\hline 18 & 262144 & 97 & 34 & 74 & 8 \\
\hline
\end{tabular}

Tabela 7.4: busca sub-ótima em instâncias difíceis; os limiares para cada teste foram obtidos através do pré-processamento exibido na tabela 7.3.

instâncias de mesmo tamanho. Para cada instância de cada grupo e para cada algoritmo, armazenamos o tempo necessário para computar todas as chamadas da função custo e o tempo total da execução do algoritmo. Finalmente, calculamos as médias desses dois tempos, para cada grupo de instância e para cada algoritmo. Nas figuras 7.5(a), 7.5(b) e 7.5(c) mostramos histogramas do tempo computacional (em logaritmo na base 2 mili-segundos) requeridos por UCS, UBB e PFS, respectivamente, obtidos a partir dos experimentos com instâncias difíceis mostrados na tabela 7.1. As barras vermelhas representam o tempo computacional gasto pela função custo, enquanto que as barras verdes são o tempo gasto nas ações remanescentes de cada algoritmo.

Por um lado, UCS gasta muito mais tempo computacional em tarefas que não envolvem o cálculo da função custo. Por outro lado, UBB e, em menor grau, PFS, gastam aproximadamente a mesma quantidade de tempo para computar a função custo e realizar outras tarefas. UCS requer mais tempo computacional total que os demais algoritmos, porém passa menos tempo computando a função custo.

Para avaliarmos a performance dos algoritmos nos experimentos de projeto de W-operadores, 


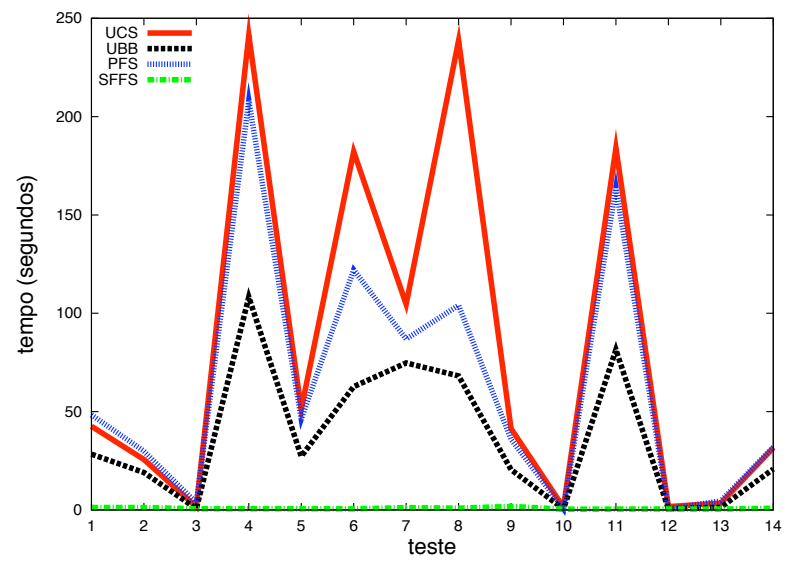

(a)

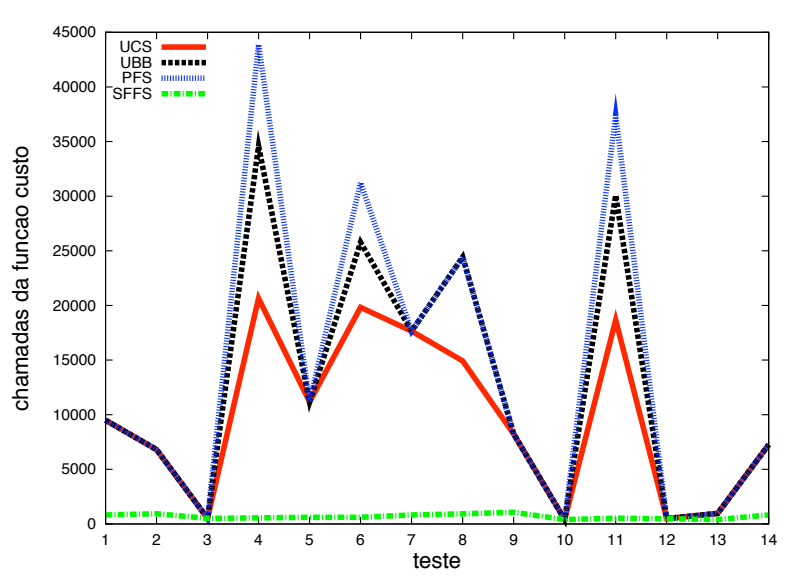

(b)

Figura 7.4: comparação entre UCS, UBB, PFS e SFFS, no experimento sub-ótimo de projeto de $W$ operador. Na figura 7.4(a) é mostrada uma comparação de tempo médio que os algoritmos gastaram para resolver cada teste; já na figura \%.4(b) é exibido o número médio de nós computados pelos algoritmos para os mesmos testes.

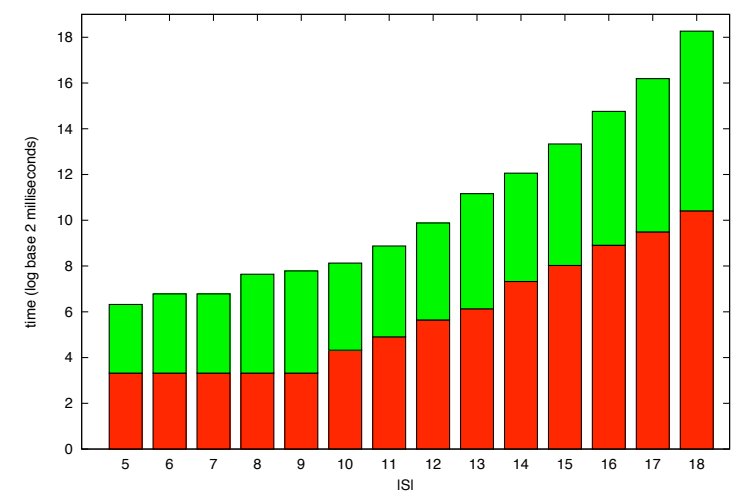

(a)

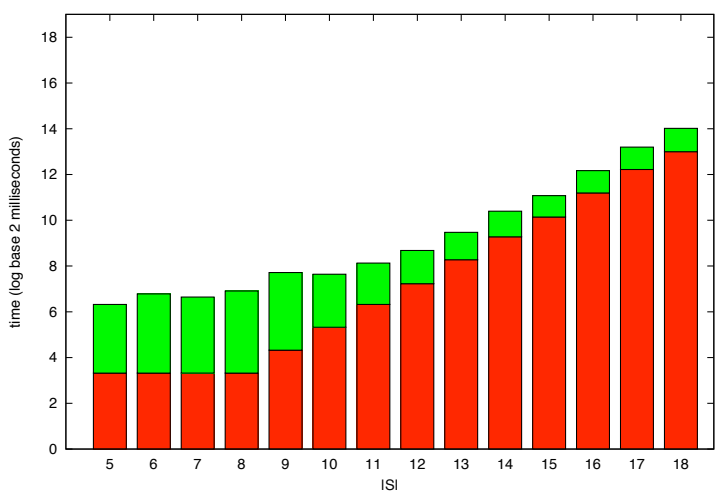

(b)

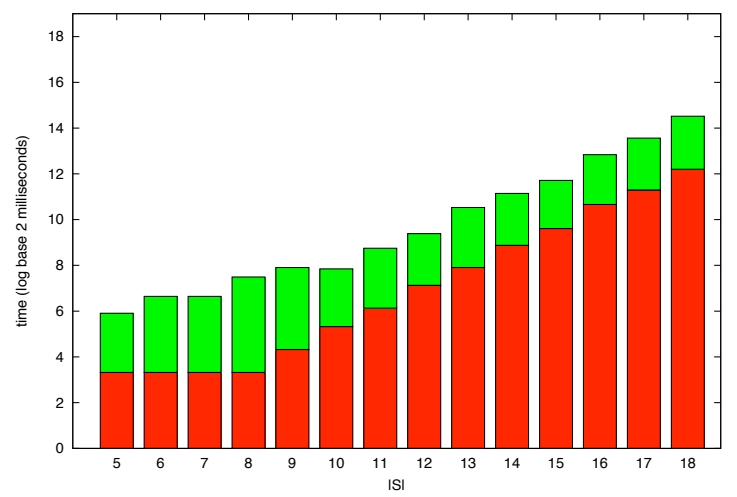

(c)

Figura 7.5: comparação entre os tempos computacionais (em log base 2 mili-segundos) requeridos por UCS (figura 7.5(a)), UBB (figura 7.5(b)) e PFS (figura 7.5(c)), nas instâncias difíceis mostradas na tabela 7.1. As barras vermelhas representam o tempo gasto em cálculo da função custo, enquanto que as barras verdes representam o tempo gasto nas ações remanescentes de cada algoritmo. O algoritmo UCS sempre exigiu mais tempo computacional em tarefas que não envolvem o cálculo da função custo; por outro lado, o fenômeno oposto ocorreu com os demais algoritmos. 


\begin{tabular}{|c|c|c|c|c|c|}
\hline \multirow[t]{2}{*}{ \# Teste } & \multicolumn{4}{|c|}{ \# Nós computados } & \multirow[t]{2}{*}{ Limiar } \\
\hline & $\mathrm{UCS}$ & UBB & PFS & SFFS & \\
\hline 1 & 198 & 7631 & $\underline{9504}$ & 396 & 9504 \\
\hline 2 & 218 & 5841 & $\underline{6804}$ & 931 & 6804 \\
\hline 3 & 61 & 96 & 197 & 507 & 507 \\
\hline 4 & 20815 & 34574 & $\underline{43882}$ & 564 & 43882 \\
\hline 5 & 254 & $\underline{11176}$ & 75 & 615 & 11176 \\
\hline 6 & 19643 & $\overline{25773}$ & $\underline{32072}$ & 615 & 32072 \\
\hline 7 & 64 & 13894 & 17649 & 827 & 17649 \\
\hline 8 & 166 & $\underline{24430}$ & 53 & 929 & 24430 \\
\hline 9 & 833 & 6730 & $\underline{8228}$ & 1070 & 8228 \\
\hline 10 & $\underline{401}$ & 64 & 100 & 295 & 401 \\
\hline 11 & $1 \overline{679}$ & 30080 & $\underline{39332}$ & 534 & 39332 \\
\hline 12 & 152 & 412 & $\underline{485}$ & 298 & 485 \\
\hline 13 & 99 & 899 & $\underline{965}$ & 298 & 965 \\
\hline 14 & 580 & 6318 & $7 \overline{259}$ & 827 & 7259 \\
\hline Média & 4440,21 & 11994,14 & 11900,36 & 621,86 & $\overline{14478,14}$ \\
\hline
\end{tabular}

Tabela 7.5: pré-processamento que produz os limiares para a busca sub-ótima no projeto de $W$-operador.

executamos os algoritmos sobre as 14 instâncias de dados reais e armazenamos, para cada instância e para cada algoritmo, o tempo necessário para computar as chamadas da função custo e o tempo exigido por toda a execução do algoritmo. Nas figuras 7.6(a), 7.6(b) e 7.6(c) mostramos histogramas do tempo necessário de computação (em segundos) exigido por UCS, UBB e PFS, respectivamente, na estimação dos $14 \mathrm{~W}$-operadores mostrados na tabela 7.2. Assim como nos histogramas dos experimentos com instâncias difíceis, as barras vermelhas representam o tempo computacional gasto pela função custo, enquanto que as barras verdes são o tempo gasto nas ações remanescentes de cada algoritmo.

Por um lado, UCS gasta muito mais tempo computacional em tarefas que não envolvem o cálculo da função custo. Por outro lado, UBB e, em menor grau, PFS, gastam aproximadamente a mesma quantidade de tempo para computar a função custo e realizar outras tarefas. UCS requer um pouco mais de tempo computacional total que os demais algoritmos, porém a diferença entre eles neste experimento é bem menor do que a verificada no experimento com instâncias difíceis; isso se deve devido ao maior tempo médio necessário para computar a função custo. Este resultado implica em que a utilização do algoritmo UCS em instâncias de W-operador mais pesadas pode tornar este algoritmo mais competitivo em relação aos demais algoritmos. Para verificar este fato, executamos os algoritmos UCS e UBB sobre as mesmas 14 instâncias de dados reais, porém utilizando 1152 amostras (i.e., o dobro de amostras em relação aos experimentos anteriores), o que acarreta em chamadas da função custo computacionalmente mais caras. Na tabela 7.7 apresentamos esse experimento ótimo: dos 14 testes, UCS foi mais rápido que UBB em 10 deles; além disso, UCS computou menos vezes a função custo em todos os testes.

Uma propriedade que foi verificada empiricamente nas tabelas 7.4 e 7.6 é que a velocidade de convergência de UCS é muito maior do que as verificadas nos algoritmos UBB, PFS e SFFS. Esta propriedade tem potencial para ser aproveitada para o desenvolvimento de melhores algoritmos ótimos de busca, embora não tenha impacto na análise de complexidade do algoritmo UCS.

\subsubsection{Análise da complexidade de tempo computacional}

Como provamos no capítulo 3, o problema U-curve (Problema 3.1.1) é NP-difícil. Dessa forma, uma vez que UCS, PFS e UBB são algoritmos ótimos de busca na resolução do problema U-curve, é esperado que eles tenham uma complexidade de tempo que é exponencial no tamanho no grau 


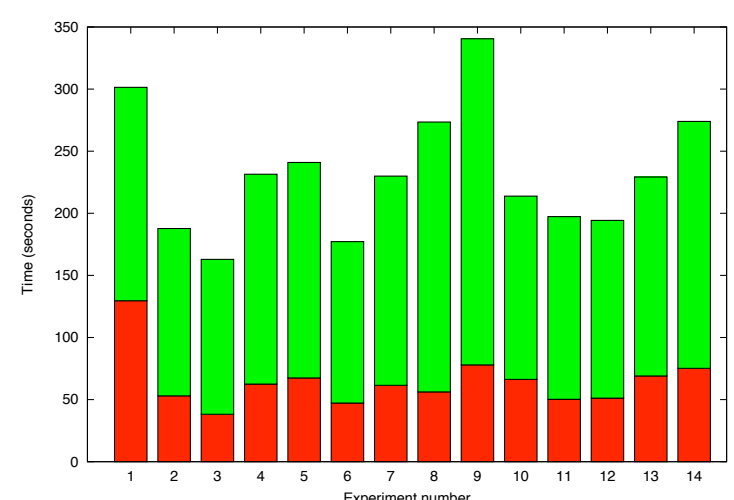

(a)

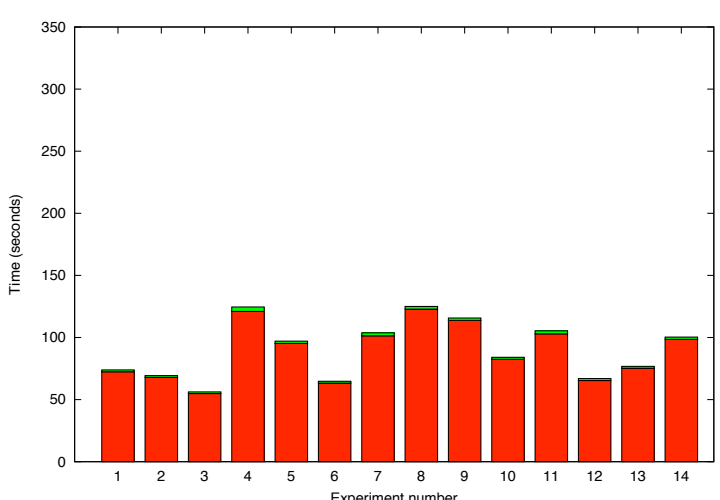

(b)

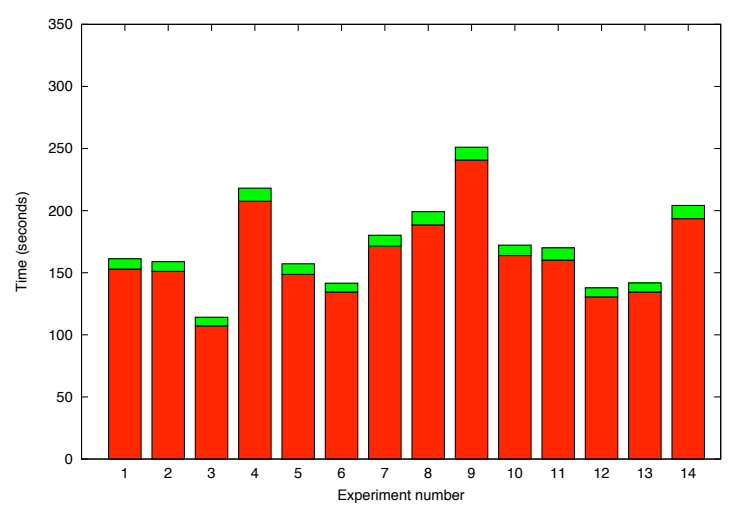

(c)

Figura 7.6: comparação entre os tempos computacionais (em segundos) requeridos por UCS (figura \%.6(a)), UBB (figura 7.6(b)) e PFS (figura 7.6(c)), nos experimentos de projeto de W-operador mostrados na tabela 7.2. As barras vermelhas representam o tempo gasto em cálculo da função custo, enquanto que as barras verdes representam o tempo gasto nas ações remanescentes de cada algoritmo.

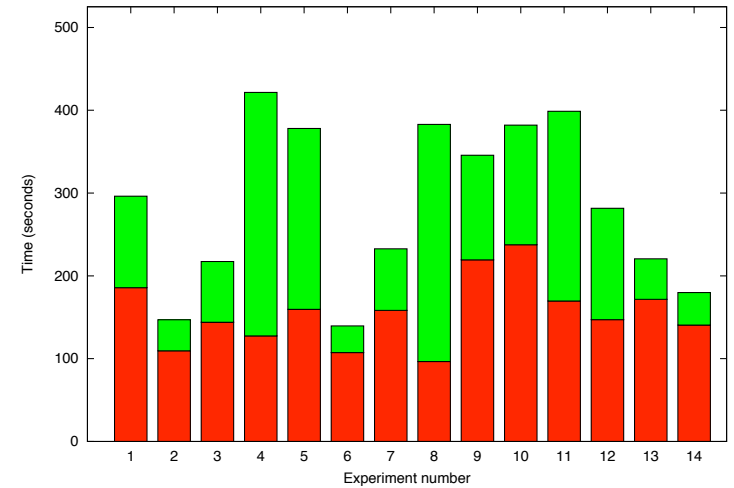

(a)

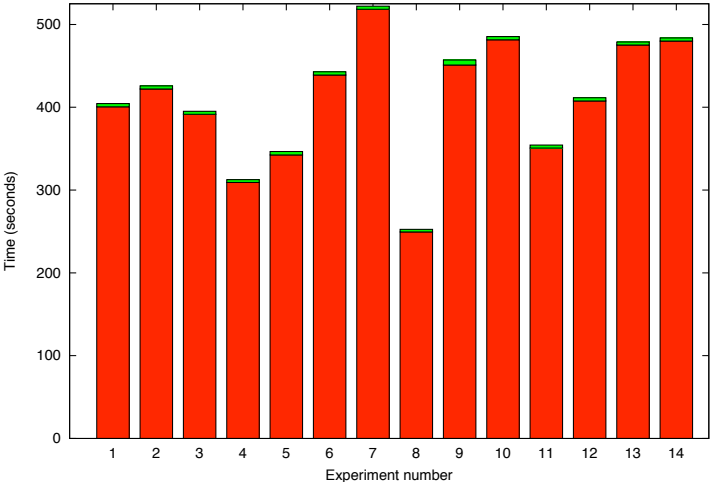

(b)

Figura 7.7: comparação entre os tempos computacionais (em segundos) requeridos por UCS (figura 7.7(a)) $e$ UBB (figura 7.7(b)), no experimento de projeto de $W$-operador mostrados na tabela 7.7. Neste experimento, cada instância utilizou o dobro de amostras em relação aos experimentos utilizados para produzir os histogramas 7.6(a) e \%.6(b); observe que para vários testes UCS realizou a sua execução em menos tempo em relação ao algoritmo $\mathrm{UBB}$. 


\begin{tabular}{|c|c|c|c|c|c|c|c|c|c|}
\hline \multirow{2}{*}{ \# Teste } & \multicolumn{4}{|c|}{ Tempo (seg) } & \multicolumn{5}{|c|}{ \# Nós computados } \\
\hline & $\mathrm{UCS}$ & UBB & PFS & SFFS & \multicolumn{2}{|c|}{ UCS } & $\mathrm{UBB}$ & PFS & SFFS \\
\hline 1 & 42,56 & 28,38 & 48,39 & 1,22 & \multicolumn{2}{|c|}{9504} & 9504 & 9504 & 827 \\
\hline 2 & 25,63 & 19,04 & 29,91 & 1,26 & \multicolumn{2}{|c|}{6804} & 6804 & 6804 & 931 \\
\hline 3 & 1,50 & 0,70 & 3,63 & 0,62 & \multicolumn{2}{|c|}{507} & 507 & 507 & 507 \\
\hline 4 & 241,30 & 108,28 & 208,00 & 0,64 & \multicolumn{2}{|c|}{20649} & 34574 & 43882 & 564 \\
\hline 5 & 51,22 & 27,25 & 46,34 & 0,63 & \multicolumn{2}{|c|}{11176} & 11176 & 11176 & 615 \\
\hline 6 & 182,19 & 62,73 & 122,06 & 0,58 & \multicolumn{2}{|c|}{19826} & 25773 & 31250 & 615 \\
\hline 7 & 104,60 & 74,79 & 86,89 & 1,19 & \multicolumn{2}{|c|}{17649} & 17649 & 17649 & 827 \\
\hline 8 & 238,64 & 68,14 & 104,05 & 0,93 & \multicolumn{2}{|c|}{14922} & 24430 & 24430 & 929 \\
\hline 9 & 41,34 & 20,50 & 35,94 & 1,83 & \multicolumn{2}{|c|}{8228} & 8228 & 8228 & 1070 \\
\hline 10 & 1,36 & 0,49 & 0,50 & 0,38 & \multicolumn{2}{|c|}{401} & 401 & 401 & 401 \\
\hline 11 & 184,08 & 81,50 & 163,88 & 0,60 & \multicolumn{2}{|c|}{18668} & 30080 & 37388 & 534 \\
\hline 12 & 1,67 & 0,61 & 0,62 & 0,53 & \multicolumn{2}{|c|}{485} & 485 & 485 & 483 \\
\hline 13 & 3,51 & 1,38 & 4,60 & 0,47 & \multicolumn{2}{|c|}{965} & 965 & 965 & 403 \\
\hline 14 & 31,61 & 20,82 & 31,97 & 0,90 & \multicolumn{2}{|c|}{7259} & 7259 & 7259 & 827 \\
\hline \multirow[t]{18}{*}{ Média } & 82,23 & 36,76 & 63,34 & 0,84 & & 38,79 & 12702,5 & 14280,57 & 680,93 \\
\hline & & \multirow{2}{*}{\multicolumn{2}{|c|}{ \# Teste }} & \multicolumn{4}{|c|}{ \# Melhor solução } & & \\
\hline & & & & UCS & $\mathrm{UBB}$ & $\mathrm{PF}_{5}$ & $\overline{\text { SFFS }}$ & & \\
\hline & & 1 & & 1 & 1 & 1 & 1 & & \\
\hline & & 2 & & 1 & 1 & 1 & 0 & & \\
\hline & & 3 & & 0 & 1 & 1 & 0 & & \\
\hline & & 4 & & 1 & 1 & 1 & 1 & & \\
\hline & & 5 & & 1 & 0 & 0 & 0 & & \\
\hline & & 6 & & 1 & 1 & 1 & 1 & & \\
\hline & & 7 & & 1 & 1 & 0 & 0 & & \\
\hline & & 8 & & 1 & 1 & 0 & 0 & & \\
\hline & & 9 & & 1 & 0 & 0 & 0 & & \\
\hline & & 10 & & 1 & 0 & 0 & 0 & & \\
\hline & & 11 & & 1 & 1 & 1 & 1 & & \\
\hline & & 12 & & 1 & 1 & 1 & 0 & & \\
\hline & & 13 & & 1 & 0 & 0 & 0 & & \\
\hline & & 14 & & 1 & 0 & 0 & 0 & & \\
\hline & & Médi & & 0,93 & 0,64 & 0,5 & 0,29 & & \\
\hline
\end{tabular}

Tabela 7.6: busca sub-ótima no projeto de $W$-operador; os limiares para cada teste foram obtidos através do pré-processamento exibido na tabela 7.5.

$n$ do reticulado Booleano que define o espaço de busca. Portanto, é esperado que a análise de complexidade de tempo desses algoritmos forneça um limiar superior justo que é exponencial em $n$.

$\mathrm{Na}$ análise de complexidade de tempo dos algoritmos deste trabalho, assumimos que qualquer chamada da função custo utilizada exige $O(f(n))$ unidades de tempo, limiar no qual $f(n)$ é definida de acordo com a função custo do problema explorado, o que implica que as análises de complexidade de tempo dos algoritmos tornam-se independentes de um tipo específico de função custo.

Como mostramos no capítulo 4, o algoritmo UCS consome $O\left(\left(f(n)+n^{2}\right) 2^{n}\right)$ unidades de tempo. A análise de complexidade de tempo do algoritmo UBB foi apresentada na seção 6.1. UBB requer $O\left((f(n)+n) 2^{n}\right)$ unidades de tempo. Finalmente, como mostramos na seção 6.2 , o algoritmo PFS consome $O\left(\left(f(n)+n^{2}\right) 2^{n}\right)$ unidades de tempo.

Entretanto, na prática, o limite superior pode ser menor do que uma função exponencial em $n$. Por isso, dizemos que, UCS requer $O\left(f(n) m+n^{2} u^{2}\right)$, no qual $m$ é o número de vezes que a função 


\begin{tabular}{|c|c|c|c|c|}
\hline \multirow[t]{2}{*}{ Instância } & \multicolumn{2}{|c|}{ Tempo (seg) } & \multicolumn{2}{|c|}{ \# Nós computados } \\
\hline & UCS & UBB & UCS & UBB \\
\hline 1 & 296,2 & 404,4 & $\underline{19023}$ & 63779 \\
\hline 2 & $\overline{146,9}$ & 425,8 & 9114 & 64406 \\
\hline 3 & 217,3 & 395,1 & 12401 & 62718 \\
\hline 4 & 421,5 & 312,6 & $\underline{25622}$ & 60265 \\
\hline 5 & 378,0 & 346,5 & $\underline{24690}$ & 62484 \\
\hline 6 & 139,5 & $\overline{442,7}$ & $\underline{7682}$ & 65197 \\
\hline 7 & 232,7 & 522,0 & $\underline{13464}$ & 64755 \\
\hline 8 & 382,8 & 252,5 & $\underline{24553}$ & 58652 \\
\hline 9 & 345,6 & 457,0 & $\underline{18461}$ & 63461 \\
\hline 10 & $\overline{382,0}$ & 485,3 & $\underline{19152}$ & 63966 \\
\hline 11 & $\overline{398,7}$ & 354,3 & $\underline{22752}$ & 61183 \\
\hline 12 & $\underline{235,8}$ & $\overline{414,4}$ & $\underline{14017}$ & 64395 \\
\hline 13 & $\overline{220,6}$ & 478,8 & $\underline{12118}$ & 64700 \\
\hline 14 & 179,7 & 483,6 & 9291 & 65469 \\
\hline Média & 284,1 & 412,5 & $\underline{16595}$ & 63245 \\
\hline
\end{tabular}

Tabela 7.7: Comparação entre os algoritmos UCS e UBB, durante num experimento ótimo de projeto de $W$-operadores utilizando as mesmas 14 instâncias de dados reais utilizadas para produzir a tabela \%.2, porém empregando o dobro de amostras (1152) em relação aos experimentos anteriores (576).

custo é computada e $u$ é proporcional a $m$ mais o número de verificações se o espaço de busca é não-vazio. UBB consome $O((f(n)+n) b)$, no qual $b$ é o número de vezes que a função custo é computada e é proporcional ao número de elementos visitados. Por fim, PFS exige $O\left(\left(f(n)+n^{2}\right) p\right)$, em que $p$ é o número de vezes que a função custo é computada e também é proporcional ao número de elementos visitados.

Nos experimentos com instâncias difíceis apresentados na seção 7.2.1, $f(n)=O(n)$, o que implica que o tempo computacional assintótico de UCS, UBB e PFS nesses experimentos foram, respectivamente, $O\left(n m+n^{2} u^{2}\right), O((n+n) b)=O(n b)$ e $\left.O\left(n+n^{2}\right) p\right)=O\left(n^{2} p\right)$. Nos experimentos ótimos de tamanho $2^{18}$ mostrados na tabela 7.1, o número de vezes que a função custo foi computada por UCS, UBB e PFS foi, respectivamente, 21991, 141241 e 79790. Ademais, o número médio de iterações do UCS foi de 44735 e o número médio de vezes que a sub-rotina DFS foi chamada foi de 1535. Então, temos que: $n=18, b=141241, p=79790$ e $u=k-j+m=44735-1535+65191=$ 108391. Dessa forma, UCS exigiu um tempo computacional que é proporcional a:

$$
\begin{aligned}
n m+n^{2} u^{2} & =18 \times 21991+18^{2} \times 108391^{2} \\
& >395 \times 4 \times 10^{9} \\
& >1 \times 10^{12} ;
\end{aligned}
$$

por outro lado, UBB exigiu um tempo computacional proporcional a:

$$
\begin{aligned}
n b & =18 \times 141241 \\
& <3 \times 10^{6},
\end{aligned}
$$

e PFS exigiu um tempo computacional proporcional a:

$$
\begin{aligned}
n^{2} p & =18^{2} \times 79790 \\
& <3 \times 10^{7} .
\end{aligned}
$$

Estes números são compatíveis com os resultados observados na tabela 7.1. Para $|S|=18$, o tempo 
médio em segundos exigido por UCS, UBB e PFS foram 315, 21, 16, 58 e 23, 44, respectivamente.

Nos experimentos com dados de projeto de W-operador apresentados na seção 7.2.2, $f(n)=$ $\Omega\left(2^{n}\right)$, uma vez que a estimação da entropia condicional média é feita utilizando um histograma contendo as distribuições de cada um dos $2^{n}$ subconjuntos de $S$. Todavia, na prática, $f(n)$ pode ter uma complexidade de tempo assintótico diferente, haja vista que é preciso levar em consideração a complexidade do algoritmo que implementa o cálculo da entropia condicional média e também o número de amostras, o que pode levar a limites superiores inferiores a $2^{n}$. Como é demonstrado no apêndice $\mathrm{C}$, o limite superior para a função custo neste experimento é $O(n l \log l)$, no qual $l$ é o mínimo entre $2^{n}$ e o número de amostras. Portanto, o tempo assintótico computacional de UCS, UBB e PFS nestes experimentos foram, respectivamente, $O\left((n l \log l) m+n^{2} u^{2}\right)$, $O((n l \log l+n) b)=O((n l \log l) b)$ e $O\left(\left(n l \log l+n^{2}\right) p\right)$. No experimento ótimo apresentado na tabela 7.2, o número médio de vezes que a função custo foi computada por UCS, UBB e PFS foram, respectivamente, 20497, 30057 e 37254 . Ademais, a média do número de iterações de UCS foi de 19623, e o número médio de vezes que a sub-rotina DFS foi chamada foi de 1665. Então nós temos que $n=16, l=\min \left\{2^{16}, 576\right\}=576, b=30057, p=37254$ e $u=19623-1665+20497=38455$. Dessa forma, neste experimento, UCS exigiu um tempo computacional que é proporcional a:

$$
\begin{aligned}
\left(n l \log _{2} l\right) m+n^{2} u^{2} & =\left(16 \times 576 \times \log _{2} 576\right) \times 20497+16^{2} \times 38455^{2} \\
& <(16 \times 576 \times 9) \times 20497+256 \times 1,5 \times 10^{9} \\
& <82944 \times 20497+384 \times 10^{9} \\
& <4 \times 10^{9}+384 \times 10^{9} \\
& <40 \times 10^{10}
\end{aligned}
$$

por outro lado, UBB exigiu um tempo computacional proporcional a:

$$
\begin{aligned}
\left(n l \log _{2} l\right) b & =\left(16 \times 576 \times \log _{2} 576\right) \times 30057 \\
& >(16 \times 576 \times 9) \times 30057 \\
& =82944 \times 30057 \\
& >2,4 \times 10^{9} \\
& >0,2 \times 10^{10} .
\end{aligned}
$$

e PFS exigiu um tempo computacional proporcional a:

$$
\begin{aligned}
\left(n l \log _{2} l+n^{2}\right) p & =\left(16 \times 576 \times \log _{2} 576+16^{2}\right) \times 37254 \\
& >(16 \times 576 \times 9+256) \times 37254 \\
& =(82944+256) \times 37254 \\
& >3 \times 10^{9} \\
& >0,3 \times 10^{10} .
\end{aligned}
$$

Estes números são compatíveis com os resultados observados na tabela 7.2. O tempo médio em segundos exigido por UCS, UBB e PFS foram 232, 45, 90, 26 e 171, 98, respectivamente.

A seguir, verificaremos a relação entre a análise teórica e os resultados obtidos nos experimentos ótimos com instâncias difíceis e de projeto de W-operadores.

- Simulação de instâncias difíceis

- Na figura 7.5(a) mostramos que UCS exige muito mais tempo computacional para tarefas que não envolvem computar a função custo. Este fato é explicado pela função custo ser linear em $n$, o que implica que a complexidade de tempo computacional de UCS é $O\left(n m+n^{2} u^{2}\right)$. O primeiro termo da expressão é equivalente ao tempo que o algoritmo passa computando a função custo, enquanto que o segundo é equivalente ao tempo que 
o mesmo cumpre outras tarefas. Uma vez que o segundo termo é muito maior do que o primeiro, a maior parte do tempo computacional de UCS é gasta em outras tarefas;

- Na figuras 7.5(b) e 7.5(c) mostramos que UBB e PFS gastam aproximadamente o mesmo tempo computacional computando a função custo e realizando outras tarefas, embora UBB gaste menos tempo em outras tarefas do que o PFS. Este fato também é explicado pela função custo ser linear em $n$, o que implica que a complexidade de tempo computacional de UBB e de PFS é $O(n b+n b)$ e $O\left(n p+n^{2} p\right)$, respectivamente. Novamente, o primeiro termo da expressão é equivalente ao tempo que o algoritmo passa computando a função custo, enquanto que o segundo é equivalente ao tempo que o mesmo cumpre outras tarefas. No caso de UBB, ambos são equivalentes, enquanto que no caso de PFS o segundo termo é maior do que o primeiro, algo intermediário entre o controle de UCS e de UBB;

- Na verificação de complexidade de tempo de UCS, UBB e PFS, a diferença na ordem de magnitude de seus tempos computacionais $\left(1 \times 10^{12}, 3 \times 10^{6}\right.$ e $3 \times 10^{7}$, respectivamente) é devido ao fato do segundo termo da expressão de complexidade de tempo de UCS (i.e., o termo que corresponde às tarefas que não envolvem computar a função custo) ter duas variáveis com fator quadrático, enquanto que a expressão de UBB tem duas variáveis lineares e a expressão de PFS tem uma variável linear e outra quadrática. Ademais, a função custo processada é linear em $n$, portanto a mesma é absorvida pelo segundo termo das expressões de complexidade de tempo de PFS e (principalmente) de UCS.

\section{- Projeto de $W$-operadores}

- Na figura 7.6(a) mostramos que UCS exige mais tempo computacional em tarefas que não envolvem o cálculo da função custo. Este fato é explicado pelo fato da função custo processada ser a entropia condicional média penalizada descrita em Apêndice $\mathrm{C}$, o que implica que a complexidade de tempo assintótico de UCS é $O\left((n l \log l) m+n^{2} u^{2}\right)$. Uma vez que o segundo termo é maior que o primeiro, a maior parte do tempo computacional de UCS é gasta na realização de outras tarefas, embora numa proporção menor do que a verificada no experimento com instâncias difíceis;

- Nas figuras 7.6(b) e 7.6(c) mostramos que UBB e PFS gastam mais tempo computando a função custo. Este fato é explicado pelo fato da função custo processada ser a entropia condicional média penalizada descrita no apêndice $\mathrm{C}$, o que implica que a complexidade de tempo assintótico de UBB e de PFS é $O((n l \log l) b+n b)$ e $O\left((n l \log l) p+n^{2} p\right)$, respectivamente. Uma vez que o primeiro termo é maior do que o segundo, a maior parte do tempo computacional de UBB e de PFS, daquele mais do que deste, é gasta para computar a função custo.

- Na verificação de complexidade de tempo de UCS, UBB e PFS, a similaridade na ordem de magnitude de seus consumos de tempo $\left(40 \times 10^{10}, 0.2 \times 10^{10}\right.$ e $0.3 \times 10^{10}$, respectivamente ) é devido ao fato da função custo processada ter uma maior ordem de complexidade, o que implica que na forte contribuição da mesma para o primeiro termo de todas as expressões de complexidade de tempo. Em particular, a ordem do primeiro termo da expressão de complexidade de tempo de UCS se aproxima da ordem de magnitude do segundo termo. Ademais, o segundo termo da expressão de complexidade de tempo de UBB é linear em $n$, e portanto é absorvida pelo primeiro termo; o mesmo, em menor grau, ocorre com o segundo termo da expressão de PFS. 


\section{Capítulo 8}

\section{Relaxamento do problema U-curve}

O relaxamento do problema U-curve consiste na generalização do problema de minimização de funções custo decomponíveis em curvas em U (problema 3.1.1). Um tipo de generalização são as funções custo parcialmente decomponíveis em curvas em U, que serão formalizadas neste capítulo. Apresentaremos também duas funções custo que são parcialmente decomponíveis em curvas em U, sendo que uma delas é de interesse prático no contexto de Reconhecimento de Padrões. Por fim, mostraremos que os algoritmos UCS, UBB e PFS resolvem esta versão generalizada do problema U-curve.

\subsection{Funções parcialmente decomponíveis em curvas em U}

Sejam $S$ um conjunto e $c$ uma função custo. Dizemos que $c$ é parcialmente decomponível em curvas em U se, para cada cadeia $\mathcal{A}$ de $\mathcal{P}(S)$ que contenha um elemento de $\mathcal{P}(S)$ de custo mínimo, a restrição de $c$ em $\mathcal{A}$ descreve uma curva em $\mathrm{U}$, ou seja, para quaisquer $A_{1}, A_{2}, A_{3} \in \mathcal{A}$, $A_{1} \subseteq A_{2} \subseteq A_{3}$ implica que $c\left(A_{2}\right) \leq \max \left\{c\left(A_{1}\right), c\left(A_{3}\right)\right\}$. A partir desta definição, podemos elaborar o seguinte problema de busca.

Problema 8.1.1. (problema U-curve parcial) Dado um conjunto $S$ de objetos e uma função custo c parcialmente decomponivel em curvas em $U$, encontrar um subconjunto $X \subseteq S$, tal que $c(X)$ seja minimo.

Toda função custo decomponível em curvas em U também é uma função custo parcialmente decomponível em curvas em U; porém, a recíproca não é verdadeira: na figura 8.1 apresentamos um reticulado Booleano $(\mathcal{P}(S), \subseteq)$ com uma função custo $c$ parcialmente decomponível em curvas em U; observe que a restrição de $c$ sobre a cadeia $\{000,010,110,111\}$ não descreve uma curva em U.

Agora faremos uso da distância de Hamming, introduzida em 1950 por Hamming [Ham50], para mostrarmos uma definição de função custo parcialmente decomponível em curvas em U.

Definição 8.1.2. Seja $S$ um conjunto finito e não-vazio. Seja $M$ um conjunto de $\mathcal{P}(S)$. A função custo da distância de Hamming é uma função custo c definida como:

$$
c(X)=|M-X|+|X-M|, \text { para todo } X \in \mathcal{P}(S) .
$$

Na figura 8.1 temos um exemplo de reticulado Booleano $(\mathcal{P}(S), \subseteq)$ de grau três, no qual $c$ é uma função custo da distância de Hamming. Como provaremos a seguir, este tipo de função é parcialmente decomponível em curvas em U.

Teorema 8.1.3. Sejam $S$ um conjunto finito e não-vazio e c uma função custo da distância de Hamming. c é parcialmente decomponivel em curvas em $U$.

Demonstração. Inicialmente verificamos que $c(M)=|M-M|+|M-M|=0+0=0$, e que portanto que $M$ é mínimo em $\mathcal{P}(S)$. Observe ainda que $M$ é o único mínimo em $\mathcal{P}(S)$, pois qualquer outro elemento desse conjunto tem distância de Hamming maior ou igual a um. 


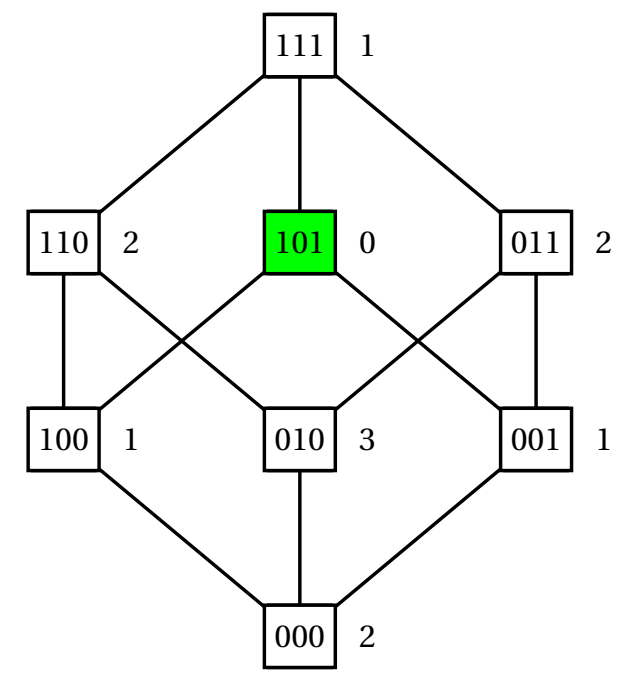

Figura 8.1: um reticulado Booleano de grau 3, com uma função custo da distância de Hamming. Neste caso, $M=101$. Observe que c é uma função custo parcialmente decomponível em curvas em $U$; todavia, $c$ não é uma função custo decomponível em curvas em $U$.

Agora, precisamos demonstrar que toda cadeia maximal que contenha o elemento $M$ é decomponível em curvas em U. Seja $\left\{A_{1}, \ldots, A_{|S|+1}\right\}$ uma cadeia que contenha $M$, tal que $A_{1} \subset \ldots \subset A_{|S|+1}$ e $A_{i}=M$. Observe que para qualquer elemento $A_{j}, 1<j \leq i$, vale que:

$$
\begin{aligned}
c\left(A_{j}\right) & =\left|A_{i}-A_{j}\right|+\left|A_{j}-A_{i}\right| \\
& =\left|A_{i}-A_{j}\right|+0 \\
& <\left|A_{i}-A_{j-1}\right| \\
& =c\left(A_{j-1}\right),
\end{aligned}
$$

e que para qualquer conjunto $A_{k}, i \leq k<|S|+1$, vale que:

$$
\begin{aligned}
c\left(A_{k}\right) & =\left|A_{i}-A_{k}\right|+\left|A_{k}-A_{i}\right| \\
& =0+\left|A_{k}-A_{i}\right| \\
& <\left|A_{k+1}-A_{i}\right| \\
& =c\left(A_{k+1}\right) .
\end{aligned}
$$

Portanto, concluímos que $c$ é parcialmente decomponível em curvas em U.

\subsection{Aplicação no projeto de classificadores}

Seja $S$ um conjunto finito e não-vazio. Um classificador $\psi$ de $S$ é uma função que leva valores de $\mathcal{P}(S)$ em $\{0,1\}$. Denotaremos por $\Psi$ o espaço de todos os classificadores, ou seja:

$$
\Psi=\{\psi: \psi \text { é um classificador de } S\} .
$$

Uma forma de representar um classificador $\psi$ é através do conjunto dos elementos de $\mathcal{P}(S)$ para os quais o valor de $\psi$ é igual a um; isto é, o conjunto:

$$
\mathcal{K}_{\psi}=\{X \in \mathcal{P}(S): \psi(X)=1\}
$$

define um classificador $\psi$ e é denominado núcleo de $\psi$. Segue imediatamente dessa definição que o conjunto de todos os núcleos de classificadores é igual a $\mathcal{P}(\mathcal{P}(S))$, e que existem $2^{\mid{ }^{|S|}}$ classificadores 
distintos dois a dois.

O poset $(\mathcal{P}(\mathcal{P}(S)), \subseteq)$ é um reticulado Booleano de grau $2^{|S|}$ no qual o maior e o menor elemento são, respectivamente, $\emptyset$ e $\mathcal{P}(S)$, e a soma e o produto são, respectivamente, a união e a interseção usuais sobre conjuntos. Agora vamos definir uma função custo chamada de função custo do erro absoluto médio (MAE - Mean Absolute Error [DeG86]). O MAE é de grande interesse prático em áreas como Reconhecimento de Padrões, pois pode ser utilizada como medida de otimalidade de um classificador [Hir00, Has00]. Dessa forma, projetar um classificador significa escolher um núcleo de classificador de $S$ que tenha custo mínimo.

Definição 8.2.1. Seja $S$ um conjunto finito e não-vazio. A função custo do erro absoluto médio é uma função custo $c: \mathcal{P}(\mathcal{P}(S)) \rightarrow \mathbb{R}$ definida como:

$$
c\left(\mathcal{K}_{\psi}\right)=E[|\mathbf{y}-\psi(\mathbf{X})|] \text {, para todo } \mathcal{K}_{\psi} \in \mathcal{P}(\mathcal{P}(S)),
$$

em que $\mathbf{y}$ e $\mathbf{X}$ são variáveis aleatórias, caracterizadas pela distribuição conjunta $P(\mathbf{y}, \mathbf{X})$ e cujos espaços amostrais são, respectivamente, $\{0,1\}$ e $\mathcal{P}(S)$.

Como veremos a seguir, existe uma boa caracterização de um mínimo de um reticulado Booleano associado a uma função custo do erro absoluto médio; ela aproveita-se do fato que uma função MAE é minimizada por sua mediana (DeGroot, capítulo 4 [DeG86]).

Lema 8.2.2. Sejam $S$ um conjunto finito e não-vazio, c uma função custo do erro absoluto médio e $\mathcal{K}_{\psi_{*}} \in \mathcal{P}(\mathcal{P}(S))$ um núcleo de $\psi_{*}$. $\mathcal{K}_{\psi_{*}}$ é um minimo de $\mathcal{P}(\mathcal{P}(S))$ se e somente se $\mathcal{K}_{\psi_{*}}=\{X \in$ $\mathcal{P}(S): P(1 \mid X) \geq 0.5\}$.

Demonstração. Para todo $\mathcal{K}_{\psi} \in \mathcal{P}(\mathcal{P}(S))$ vale que:

$$
\begin{aligned}
c\left(\mathcal{K}_{\psi}\right) & =E[|\mathbf{y}-\psi(\mathbf{X})|] \\
& =\sum_{(X, y)}|y-\psi(X)| P(X, y) \\
& =\sum_{(X, y)}|y-\psi(X)| P(X) P(y \mid X) \\
& =\sum_{X} P(X) \sum_{y}|y-\psi(X)| P(y \mid X) \\
& =\sum_{X} P(X)[(0-\psi(X)) P(0 \mid X)+(1-\psi(X)) P(1 \mid X)] \\
& =\sum_{X} P(X)[\psi(X) P(0 \mid X)+(1-\psi(X)) P(1 \mid X)] .
\end{aligned}
$$

Ou seja, $c$ é minimizada se e somente se, para cada termo do somatório da equação (8.1f), temos que:

$$
\psi(X)= \begin{cases}0, & \text { se } P(0 \mid X) \geq P(1 \mid X) \\ 1, & \text { caso contrário. }\end{cases}
$$

Inicialmente consideremos que $\mathcal{K}_{\psi_{*}}$ seja igual a $\{X \in \mathcal{P}(S): P(1 \mid X) \geq 0.5\}$; como $\psi_{*}(X)=1$ implica que $P(1 \mid X) \geq 0.5 \geq P(0 \mid X)$, cada termo do somatório da equação (8.1f) é minimizado. Portanto, $\mathcal{K}_{\psi_{*}}$ é um mínimo de $\mathcal{P}\left(\mathcal{P}(S)\right.$ ). Inversamente, se $\mathcal{K}_{\psi_{*}}$ é um mínimo de $\mathcal{P}(\mathcal{P}(S)$ ), a função $c$ é minimizada e, em todos os termos do somatório da equação (8.1f), $P(1 \mid X) \geq 0.5$ implica que $\psi_{*}(X)=1$. Logo, podemos definir $\mathcal{K}_{\psi_{*}}$ como $\{X \in \mathcal{P}(S): P(1 \mid X) \geq 0.5\}$, o que encerra a demonstração.

O resultado do lema 8.2.2 fornece uma solução analítica imediata para um mínimo global, dispensando a necessidade de busca pelo reticulado por tal mínimo. Não obstante, mostraremos a seguir que as funções custo do erro absoluto médio são parcialmente decomponíveis em curvas em U. 
Teorema 8.2.3. Sejam $S$ um conjunto finito e não-vazio e c uma função custo do erro absoluto médio. c é parcialmente decomponivel em curvas em $U$.

Demonstração. Seja $\mathcal{K}_{\psi_{*}}$ um mínimo de $\mathcal{P}(\mathcal{P}(S))$. Seja $\left\{\mathcal{K}_{\psi_{1}}, \ldots, \mathcal{K}_{\psi_{\mathcal{P}(S)+1}}\right\} \subseteq \mathcal{P}(\mathcal{P}(S))$ uma cadeia que contenha $\mathcal{K}_{\psi_{*}}$, tal que $\mathcal{K}_{\psi_{1}} \subset \ldots \subset \mathcal{K}_{\psi_{\mathcal{P}(S)+1}}$ e $\mathcal{K}_{\psi_{i}}=\mathcal{K}_{\psi_{*}}$. Observe que para qualquer conjunto $\mathcal{K}_{\psi_{j}}, 1<j \leq i$, vale que:

$$
\begin{aligned}
c\left(\mathcal{K}_{\psi_{j}}\right) & =\sum_{X} P(X) \sum_{y}\left|y-\psi_{j}(X)\right| P(y \mid X) \\
& =\sum_{X \in \mathcal{P}(S)-\{Y\}} P(X) \sum_{y}\left|y-\psi_{j}(X)\right| P(y \mid X)+P(Y) \sum_{y}\left|y-\psi_{j}(Y)\right| P(y \mid Y) \\
& =\sum_{X \in \mathcal{P}(S)-\{Y\}} P(X) \sum_{y}\left|y-\psi_{j}(X)\right| P(y \mid X)+P(Y) P(0 \mid Y) \\
\leq & \sum_{X \in \mathcal{P}(S)-\{Y\}} P(X) \sum_{y}\left|y-\psi_{j}(X)\right| P(y \mid X)+P(Y) P(1 \mid Y) \\
& =\sum_{X \in \mathcal{P}(S)-\{Y\}} P(X) \sum_{y}\left|y-\psi_{j-1}(X)\right| P(y \mid X)+P(Y) P(1 \mid Y) \\
& =\sum_{X \in \mathcal{P}(S)-\{Y\}} P(X) \sum_{y}\left|y-\psi_{j-1}(X)\right| P(y \mid X)+P(Y) \sum_{y}\left|y-\psi_{j-1}(Y)\right| P(y \mid Y) \\
& =\sum_{X} P(X) \sum_{y}\left|y-\psi_{j-1}(X)\right| P(y \mid X) \\
& =c\left(\mathcal{K}_{\psi_{j-1}}\right),
\end{aligned}
$$

em que $Y \in \mathcal{K}_{\psi_{j}}-\mathcal{K}_{\psi_{j-1}}$, ou seja, $Y$ é o único conjunto de $\mathcal{K}_{\psi_{j}}$ que não pertence a $\mathcal{K}_{\psi_{j}-1}$.

Agora precisamos justificar a desigualdade da equação (8.2d): ela ocorre devido ao fato que $P(1 \mid Y) \geq P(0 \mid Y)$, para todo $Y \in \mathcal{K}_{\psi_{j}}-\mathcal{K}_{\psi_{j-1}}$, para todo $1<j \leq i$. Tal fato pode ser demonstrado por contradição: suponhamos, por um instante, que $P(1 \mid Y)<P(0 \mid Y)$ para algum $Y \in \mathcal{K}_{\psi_{j}}-\mathcal{K}_{\psi_{j-1}}$. É claro que $\psi_{j}(Y)=1, \psi_{*}(Y)=1$ e $\psi_{j-1}(Y)=0$. Dessa forma, poderíamos definir um núcleo $\mathcal{K}_{\psi_{* *}}:=\mathcal{K}_{\psi_{*}}-\{Y\}$, de forma que:

$$
\begin{aligned}
c\left(\mathcal{K}_{\psi_{*}}\right)= & \sum_{X} P(X) \sum_{y}\left|y-\psi_{*}(X)\right| P(y \mid X) \\
= & \sum_{X \in \mathcal{P}(S)-\{Y\}} P(X) \sum_{y}\left|y-\psi_{*}(X)\right| P(y \mid X)+P(Y) \sum_{y}\left|y-\psi_{*}(Y)\right| P(y \mid Y) \\
= & \sum_{X \in \mathcal{P}(S)-\{Y\}} P(X) \sum_{y}\left|y-\psi_{*}(X)\right| P(y \mid X)+P(Y) P(0 \mid Y) \\
> & \sum_{X \in \mathcal{P}(S)-\{Y\}} P(X) \sum_{y}\left|y-\psi_{*}(X)\right| P(y \mid X)+P(Y) P(1 \mid Y) \\
= & \sum_{X \in \mathcal{P}(S)-\{Y\}} P(X) \sum_{y}\left|y-\psi_{* *}(X)\right| P(y \mid X)+P(Y) \sum_{y}\left|y-\psi_{* *}(Y)\right| P(y \mid Y) \\
= & \sum_{X} P(X) \sum_{y}\left|y-\psi_{* *}(X)\right| P(y \mid X) \\
= & c\left(\mathcal{K}_{\psi_{* *}}\right),
\end{aligned}
$$

o que contraria a minimalidade de $\mathcal{K}_{\psi_{*}}$.

Raciocínio simétrico ao empregado para todo conjunto $\mathcal{K}_{\psi_{j}}, 1<j \leq i$, vale para qualquer conjunto $\mathcal{K}_{\psi_{k}}, i \leq k<|\mathcal{P}(S)+1|$. Portanto, concluímos que $c$ é parcialmente decomponível em curvas em U. 


\begin{tabular}{rcccccccc}
\hline$X$ & $\psi_{1}(X)$ & $\psi_{2}(X)$ & $\psi_{3}(X)$ & $\psi_{4}(X)$ & $\psi_{5}(X)$ & $\psi_{6}(X)$ & $\psi_{7}(X)$ & $\psi_{8}(X)$ \\
\hline$\emptyset$ & 0 & 1 & 0 & 1 & 0 & 1 & 0 & 1 \\
$\{a\}$ & 0 & 0 & 1 & 1 & 0 & 0 & 1 & 1 \\
$\{b\}$ & 0 & 0 & 0 & 0 & 1 & 1 & 1 & 1 \\
$\{a, b\}$ & 0 & 0 & 0 & 0 & 0 & 0 & 0 & 0 \\
\hline \hline$X$ & $\psi_{9}(X)$ & $\psi_{10}(X)$ & $\psi_{11}(X)$ & $\psi_{12}(X)$ & $\psi_{13}(X)$ & $\psi_{14}(X)$ & $\psi_{15}(X)$ & $\psi_{16}(X)$ \\
\hline$\emptyset$ & 0 & 1 & 0 & 1 & 0 & 1 & 0 & 1 \\
$\{a\}$ & 0 & 0 & 1 & 1 & 0 & 0 & 1 & 1 \\
$\{b\}$ & 0 & 0 & 0 & 0 & 1 & 1 & 1 & 1 \\
$\{a, b\}$ & 1 & 1 & 1 & 1 & 1 & 1 & 1 & 1 \\
\hline
\end{tabular}

Tabela 8.1: todos os possíveis classificadores de $S$; como $|S|=2$, temos que $|\Psi|$ (o tamanho do espaço de classificadores) é igual a $2^{2^{|S|}}=2^{2^{2}}=16$.

\begin{tabular}{rcccccc}
\hline$X$ & $P(0 \mid X)$ & $P(1 \mid X)$ & $P(X)$ & $\psi_{1}(X)$ & $\psi_{7}(X)$ & $\psi_{13}(X)$ \\
\hline$\emptyset$ & 0.5 & 0.5 & 0.1 & 0 & 0 & 0 \\
$\{a\}$ & 0.4 & 0.6 & 0.25 & 0 & 1 & 0 \\
$\{b\}$ & 0.3 & 0.7 & 0.4 & 0 & 1 & 1 \\
$\{a, b\}$ & 0.8 & 0.2 & 0.25 & 0 & 0 & 1 \\
\hline
\end{tabular}

Tabela 8.2: todos os valores da distribuição conjunta $P(\mathbf{y}, \mathbf{X})$, acompanhados dos valores de três classificadores de $S=\{a, b\}: \psi_{1}, \psi_{7}$ e $\psi_{13}$. O núcleo do classificador $\psi_{7}$ é mínimo em $\mathcal{P}(\mathcal{P}(S))$.

\subsubsection{Um exemplo de aplicação}

Encerraremos esta seção com um toy model, um exemplo de projeto de um classificador de um pequeno conjunto com dois elementos. Sejam $S=\{a, b\}$ um conjunto e $c$ uma função custo do erro absoluto médio. A tabela 8.2 contém uma valoração da distribuição conjunta das variáveis aleatórias $\mathbf{y}$ e $\mathbf{X}$; com essa valoração, podemos determinar todos os valores de $c(X)$, em que $X$ é um núcleo de um classificador de $S$; existem $2^{2^{|S|}}=2^{2^{2}}=16$ classificadores de $S$ distintos dois a dois (tabela 8.1). A coleção dos núcleos de todos os classificadores de $S$, somada à relação usual de inclusão, constituem um reticulado Booleano de grau $2^{|S|}$ (figura 8.2).

Observe que dois núcleos, $\mathcal{K}_{\psi_{7}}$ e $\mathcal{K}_{\psi_{8}}$ são mínimos em $\mathcal{P}(\mathcal{P}(S))$. Ademais, como $c$ é uma função custo do erro absoluto médio, $c$ é parcialmente decomponível em curvas em U e qualquer cadeia que contenha $\mathcal{K}_{\psi_{7}}$ e/ou $\mathcal{K}_{\psi_{8}}$ descreve uma curva em U (figura 8.3).

\subsection{Corretude dos algoritmos UCS, UBB e PFS}

Agora demonstraremos que podemos utilizar o algoritmos introduzidos neste trabalho para realizar buscas de conjuntos mínimos em reticulados Booleanos cujas funções custo sejam parcialmente decomponíveis em curvas em U. Ou seja, mostraremos que os algoritmos UCS, UBB e PFS devolvem corretamente os mínimos globais.

Lema 8.3.1. Sejam $S$ um conjunto finito e não-vazio e c uma função custo parcialmente decomponivel em curvas em U. O algoritmo UCS devolve todos os minimos de $\mathcal{P}(S)$.

Demonstração. Inicialmente consideremos o caso em que a iteração do algoritmo UCS percorre uma cadeia que descreve uma curva em U: o algoritmo se comporta de forma idêntica ao caso em que a função custo é decomponível em curvas em U; portanto, as condições necessárias e suficientes para realizar as podas (proposições 4.2 .1 e 4.2.2) são respeitadas. 


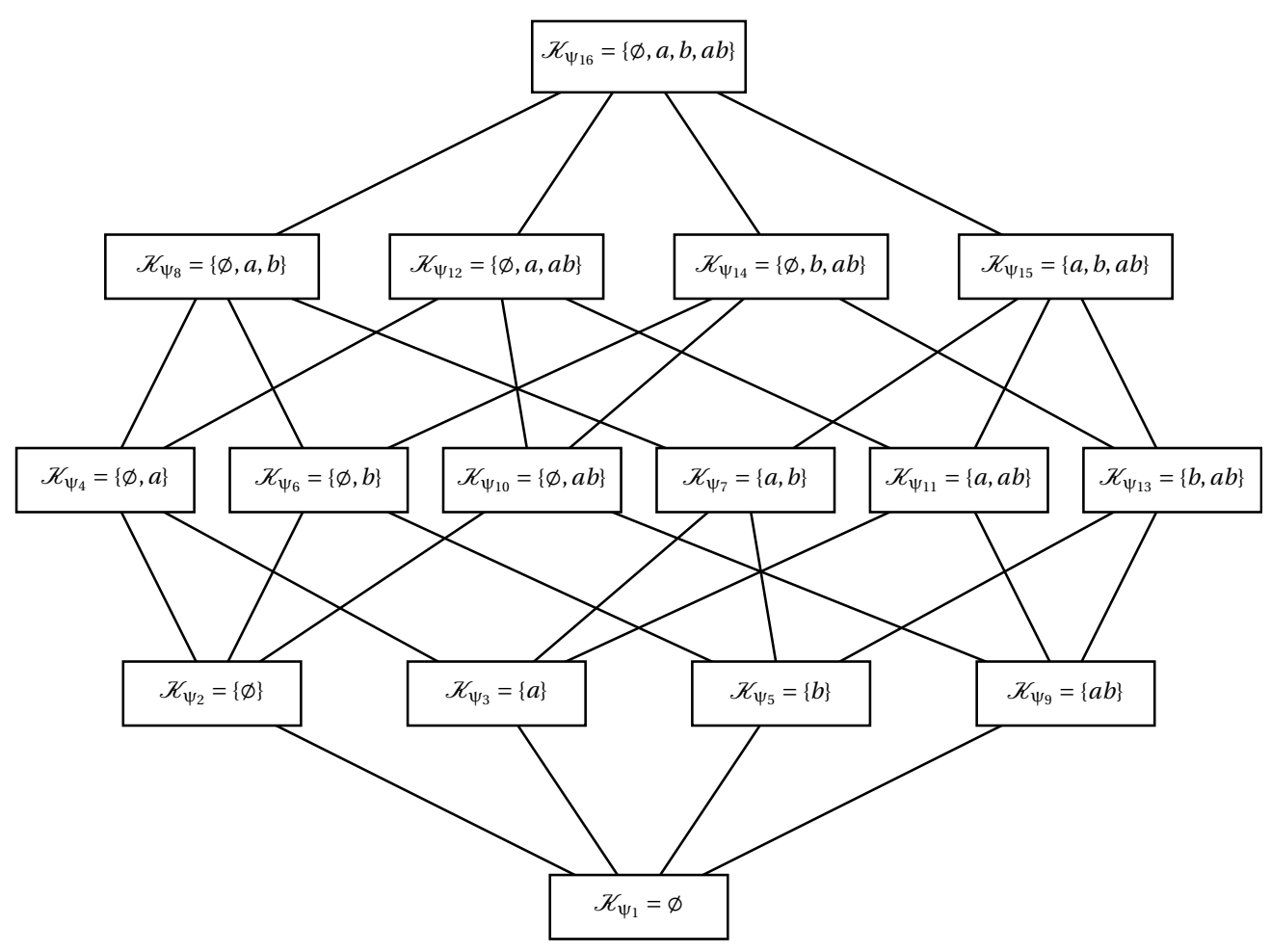

Figura 8.2: o reticulado Booleano dos núcleos de todos os classificadores de $S=\{a, b\}$.

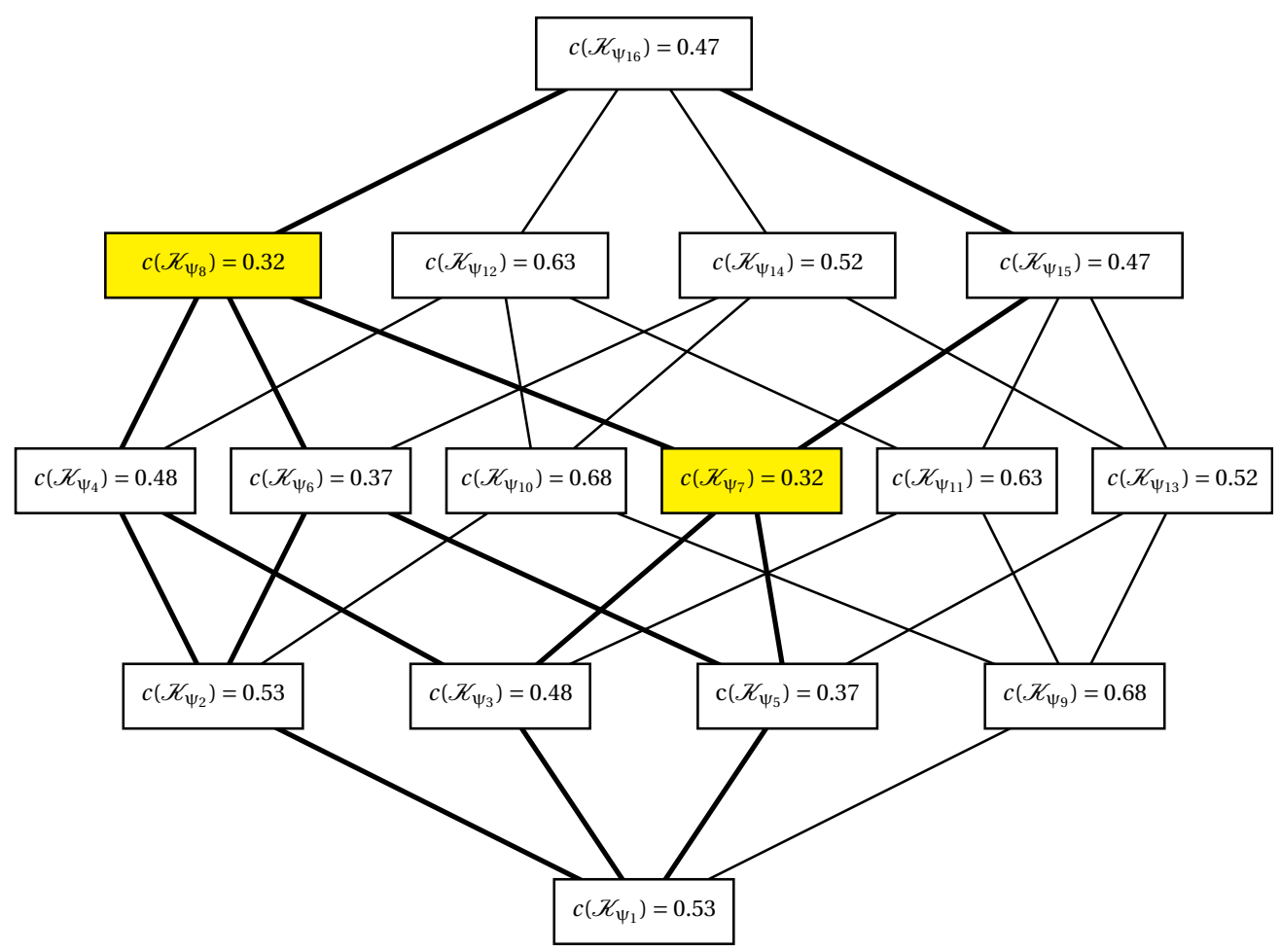

Figura 8.3: o reticulado Booleano dos núcleos de todos os classificadores de $S=\{a, b\}$, com os valores de uma função custo do erro absoluto médio que utiliza a distribuição conjunta especificada na tabela 8.2. $\mathcal{K}_{\psi_{7}}$ e $\mathcal{K}_{\psi_{8}}$ são os mínimos de $\mathcal{P}(\mathcal{P}(S))$; todas as cadeias maximais que contêm esses mínimos descrevem uma curva em $U$ e estão assinaladas com as arestas em negrito. 
Dessa forma, precisamos nos preocupar com as cadeias que não descrevem uma curva em U: ou seja, temos que garantir que a atualização das coleções de restrições não provoquem a perda de um mínimo global. Sejam $A \subset B \subset C$ subconjuntos de $S$, tais que $A$ é adjacente a $B, B$ é adjacente a $C$ e $c(B)>\max \{A, C\}$. Observe que podemos incluir $B$ nas coleções de restrições sem perigo de perder nenhum mínimo global, uma vez que, pela definição das coleções de restrições, se incluir $B$ nas mesmas implicasse em retirar um conjunto mínimo global do espaço de busca, seguiria que existe uma cadeia que contém $B$ e esse mínimo. Porém, isso não pode acontecer, pois todas as cadeias que contêm mínimos globais descrevem curvas em U (definição 8.1.2) o que contradiz a definição da cadeia que contém $B$.

Portanto, concluímos que o algoritmo UCS devolve todos dos mínimos de $\mathcal{P}(S)$.

Corolário 8.3.2. O algoritmo UCS resolve o problema de minimização de funções custo parcialmente decomponiveis em curvas em $U$.

Demonstração. Segue imediatamente do lema 8.3.1.

Agora provaremos que o algoritmo UBB resolve o problema de minimização de funções custo parcialmente decomponíveis em curvas em U.

Lema 8.3.3. Sejam $S$ um conjunto finito e não-vazio e c uma função custo parcialmente decomponivel em curvas em $U$. O algoritmo UBB devolve todos os minimos de $\mathcal{P}(S)$.

Demonstração. Inicialmente consideremos os casos em que o algoritmo UBB percorre uma cadeia do reticulado Booleano que descreva uma curva em U: o comportamento do algoritmo será idêntico ao verificado quando o mesmo é aplicado em uma instância do problema U-curve; portanto, não há risco de perder um mínimo global em uma poda.

Agora, consideremos o caso quando o algoritmo ramifica por uma cadeia que não seja decomponível em curvas em U: podar cadeias desse tipo não pode implicar em perda de mínimo global, uma vez que elementos desse tipo só estão contidos em cadeias que descrevem curvas em U.

Corolário 8.3.4. O algoritmo UBB resolve o problema de minimização de funções custo parcialmente decomponiveis em curvas em $U$.

Demonstração. Segue imediatamente do lema 8.3.3.

Finalmente, mostraremos que o algoritmo PFS também resolve o problema.

Lema 8.3.5. Sejam $S$ um conjunto finito e não-vazio e c uma função custo parcialmente decomponivel em curvas em $U$. O algoritmo PFS devolve todos os minimos de $\mathcal{P}(S)$.

Demonstração. O mesmo argumento utilizado para provar a correção de UBB na resolução deste problema serve para justificar a correção de PFS nas iterações que envolvam a floresta $\mathcal{A}$. Ademais, pelo princípio da dualidade, o mesmo resultado também vale para a floresta $\mathcal{B}$.

Corolário 8.3.6. O algoritmo PFS resolve o problema de minimização de funções custo parcialmente decomponiveis em curvas em $U$.

Demonstração. Segue imediatamente do lema 8.3.5.

\subsection{Comentários}

Esta é uma linha de pesquisa que tem potencial para modelar muitos problemas práticos em Reconhecimento de Padrões. Uma possibilidade é a modelagem de espaços de busca restritos (i.e., posets definidos a partir de um reticulado Booleano) que sejam caracterizados por uma função custo parcialmente decomponível em curvas em U. Tais espaços de busca aparecem naturalmente no projeto de classificadores: por exemplo, quando se deseja projetar um W-operador que pertença à família de operadores crescentes [Hir00], ou então à família de operadores multi-resolução 
[HJBBD02, VBHJ05]. Todavia, para que os algoritmos UCS, UBB e PFS possam ser utilizados em posets que são subespaços de um reticulado Booleano, os mesmos precisarão de algumas adaptações.

Outra possibilidade interessante seria estudar as famílias de instâncias com oscilações para as quais os algoritmos UCS, UBB e PFS sempre encontram uma solução ótima. Tais oscilações foram mencionadas por Ris e colegas [Ris08, RBMJ10], porém as suas propriedades não foram de fato definidas e provadas. 


\section{Capítulo 9}

\section{Conclusões}

Iniciaremos este capítulo de conclusões com uma revisão sobre os tópicos apresentados nesta tese, acompanhada de uma discussão sobre os resultados apresentados. Em seguida, apontaremos as principais contribuições deste trabalho. Listaremos também os trabalhos científicos que foram elaborados baseados nesta tese e que foram enviados para publicação. Finalmente, apresentaremos algumas direções que esta linha de pesquisa poderia tomar em trabalhos futuros.

\subsection{Revisão e discussão dos resultados apresentados}

Nesta tese, demonstramos que o problema de otimização U-curve é NP-difícil, Nossa demonstração foi feita através de uma redução polinomial do problema da soma de subconjuntos, um bem-conhecido problema NP-completo. Esta redução polinomial é linear no tamanho de uma instância da soma de subconjuntos, o que proporciona uma maneira eficiente de produzir instâncias "difíceis" do problema U-curve.

Visando resolver o problema U-curve, em 2010 Ris e colegas introduziram o algoritmo U-CURVE. U-CuRve é baseado na estrutura de reticulado Booleano do espaço de busca. Os princípios de funcionamento deste algoritmo são: (i) se o espaço de busca corrente não é vazio, então um elemento $M$ é empilhado em uma pilha; caso contrário, o algoritmo para; (ii) o algoritmo seleciona o topo da pilha $M$; se existe um elemento $N$ adjacente a $M$ tal que $c(N) \leq c(M)$, então $N$ é inserido na pilha; caso contrário, $M$ é desempilhado e utilizado para podar o espaço de busca corrente; (iii) se a pilha não é vazia, então o algoritmo retorna para o passo (ii); caso contrário, ele retorna ao passo (i). Apontamos que este algoritmo tem um erro na poda que leva a resultados sub-ótimos; tal erro foi apontado na figura 4.1, através de uma simulação do algoritmo.

Este trabalho introduziu um novo algoritmo, o U-CURVE-SEARCH (UCS), o qual é realmente ótimo para solucionar o problema U-curve. UCS mantém a estrutura geral de U-CURVE porém promovendo uma alteração no passo (ii): o novo algoritmo empilha elementos adjacentes a $M$ no espaço de busca corrente até que: ele encontre um elemento $N$ tal que $c(N) \leq c(M)$ (i.e., ele alcança o critério de avanço na busca em profundidade - depth-first search criterion) ou então ele explore todos os elementos adjacentes. Ademais, UCS utiliza $M$ para podar o espaço de busca corrente apenas se são atendidas condições necessárias e suficientes para realizar a poda sem o risco da perda de mínimos globais. UCS também conta com importantes melhorias:

1. o critério de avanço na busca em profundidade evita manter muitos elementos na memória, dessa forma fornecendo um melhor controle do uso de memória por parte do algoritmo;

2. uma vez que UCS atinge um elemento mais profundo, o algoritmo realiza menos podas, porém as mesmas são mais efetivas.

A ideia geral dos princípios de funcionamento do algoritmo UCS foram apresentadas através de uma simulação exibida nas figuras 4.3-4.9 . 
Realizamos alguns testes de desempenho envolvendo UCS e uma busca exaustiva descrita no apêndice B. Do ponto de vista semântico, UCS foi muito mais eficiente que a busca exaustiva, precisando, em muitos casos, calcular menos de $10 \%$ dos elementos do reticulado Booleano para encontrar uma solução ótima. Por outro lado, UCS exigiu maior tempo computacional em suas execuções. Para tentarmos descobrir como melhorar o desempenho de UCS, investigamos alguns aspectos da dinâmica deste algoritmo. Ao analisarmos a relação entre o número de iterações de uma execução UCS e o número de chamadas da sub-rotina DFS, descobrimos que o algoritmo procura por um elemento do espaço de busca corrente utilizando uma heurística muito ineficiente; este fato foi evidenciado nas figuras 4.15(a) e 4.15(b).

A constatação de que UCS não consegue encontrar de forma eficiente um elemento do espaço de busca serviu de motivação para a formalização do problema da busca de início de caminho (problema 5.1.1). Também formulamos uma variação do problema da busca de início de caminho mínimo (no caso dual, máximo), uma variação do problema que exige a minimalidade (maximalidade) do elemento a ser encontrado. Conjecturamos a complexidade do problema da busca de início de caminho e provamos que o problema da busca de caminho mínimo (máximo) é NP-difícil. Propusemos duas soluções alternativas para resolver o problema da busca de início de caminho. A primeira delas envolve a descrição do problema como um problema de Programação Linear Inteira (problema 5.2.1) e resolve-o diretamente, utilizando um algoritmo do tipo branch-and-cut. Já a segunda faz uso da versão relaxada da variação do problema que exige minimalidade (maximalidade) para obter, empregando o algoritmo Simplex, estimativas de limites inferiores e superiores da região do reticulado Booleano onde deve ser buscado um início de caminho; somente então, numa segunda etapa, é realizada a busca propriamente dita, utilizando uma nova estrutura de dados e um esquema de enumeração. Realizamos experimentos que mostraram que a primeira abordagem é computacionalmente inviável, enquanto que a segunda mostrou resultados próximos dos obtidos pelo algoritmo UCS original; tais fatos foram mostrados, respectivamente, nas tabelas 5.2 e 5.3.

Apresentamos prova de corretude, análise de complexidade de tempo e experimentos para U-CURVE-BRANCH-AND-Bound (UBB), um algoritmo ótimo do tipo branch-and-bound para abordar o problema U-curve, cujos princípios de funcionamento foram introduzidos em 2010 por Ris e colegas. UBB utiliza uma árvore $T$ como espaço de busca. $T$ é um subgrafo do diagrama de Hasse do reticulado Booleano e é construída implicitamente através de um esquema de enumeração. Durante a busca $T$ é podada utilizando o fato de que a função custo é decomponível em curvas em U: este algoritmo ramifica até que o custo de um elemento visitado comece a subir (o limiar da busca); caso isto ocorra, a árvore é podada. Constatamos, com um exemplo mostrado na figura 6.5, que UBB possui limitações em seu desempenho, sendo ineficiente quando aplicado em instâncias cujos mínimos das cadeias do reticulado Booleano encontram-se próximos das folhas de $T$. Com a motivação de corrigir esse fato, introduzimos também PoseT-Forest-SEARCh (PFS), uma generalização do algoritmo UBB. PFS trata o espaço de busca como duas florestas, sendo que ambas representam o mesmo espaço de busca corrente: a diferença entre elas é que cada uma é utilizada para percorrer as cadeias do reticulado Booleano em um sentido, o que torna o algoritmo bidirecional. Os princípios de funcionamento do algoritmo PFS foram apresentados através de uma simulação exibida nas figuras 6.8-6.14.

Realizamos um diagnóstico da qualidade dos algoritmos UCS, UBB e PFS através de experimentos com dados reais e simulados. Por um lado, UCS gasta muito tempo computacional procurando por um novo elemento do espaço de busca, o que contribui fortemente para o tempo computacional total consumido por este algoritmo. Este fato foi mostrado nas figuras 4.15(a) e 4.15(b). Por outro lado, as podas de UCS são bastante eficientes; este fato foi sugerido na tabela 7.4, a qual mostra que UCS converge de forma relativamente rápida, e também pelas figuras 7.5(a) e 7.6(a), que mostraram que UCS calcula poucas vezes a função custo ao mesmo tempo em que cobre uma grande fatia do espaço de busca. Ademais, UCS mantém o uso de memória sob controle: o maior tamanho que o grafo atinge durante a execução mantém uma pequena proporção constante no tamanho da instância; este fato foi mostrado nas figuras 4.14(a) e 4.14(b). Uma com- 
paração entre UCS, UBB, PFS e SFFS também foi feita através de experimentos com dados reais e simulados. UCS teve uma melhor performance na exploração do espaço de busca do que UBB e PFS, geralmente precisando de menos cálculo da função custo para encontrar uma solução ótima. Além disso, UCS exigiu um tempo similar aos de UBB e de PFS para encontrar soluções ótimas nos experimentos com dados reais; estes fatos foram mostrados nas tabelas 7.1 e 7.2 e discutidas na seção 7.4.1. Nos experimentos sub-ótimos, UCS teve uma melhor performance que os demais algoritmos, encontrando mais frequentemente uma melhor solução; PFS teve o segundo melhor desempenho. Além disso, a velocidade de convergência de UCS foi muito maior do que as verificadas em UBB, PFS e SFFS; estes fatos foram observados empiricamente nas tabelas 7.4 e 7.6. UBB e PFS exigiram menos tempo computacional do que UCS nos experimentos com dados simulados, nos quais o cálculo da função custo era barato; este fato foi mostrado na figura 7.5. Todavia, as figuras 4.15(a) e 4.15(b) sugeriram que a diferença entre os algoritmos é ao menos parcialmente explicada pelo fato de UCS ser ineficiente na busca de um início de caminho.

As apresentações dos algoritmos UCS, UBB e PFS incluíram as suas respectivas análises de complexidade de tempo e, no caso do UBB, também prova de corretude. Estas análises foram utilizadas para avaliarmos a consistência entre o limite superior assintótico previsto e os resultados dos experimentos. Por um lado, a avaliação dos limites superiores assintóticos para os experimentos com dados simulados, nos quais a função custo é linear em $n$, foi consistente com os resultados experimentais mostrados nas figuras 7.5(a), 7.5(b) e 7.5(c). Por outro lado, na avaliação dos limites assintóticos superiores com os experimentos de dados reais, nos quais a complexidade da função custo derivda da implementação da entropia condicional média penalizada descrita no apêndice C, apesar de ter sido verificada a correta ordem de magnitude, foi verificado que o modelo não foi acurado o bastante para verificar que o desempenho de UCS foi próximo dos de UBB e de PFS.

Finalmente, apresentamos um relaxamento (generalização) do problema U-curve, com a definição do problema U-curve parcial (problema 8.1.1), no qual nem todas as cadeias do reticulado Booleano são decomponíveis em curvas em U. Verificamos que o problema U-curve parcial modela o espaço de classificadores cuja medida de otimalidade é o erro absoluto médio - MAE; tal constatação foi ilustrada na figura 8.3. Foi demonstrado que os principais algoritmos introduzidos neste trabalho - UCS, UBB e PFS - servem para resolver esta versão relaxada do problema U-curve.

\subsection{Contribuições deste trabalho}

Listaremos agora as principais contribuições científicas e tecnológicas desta tese.

\subsubsection{Contribuições científicas}

1. Uma nova formalização do problema U-curve (problema 3.1.1), com separação semântica do problema em si da primeira solução proposta para abordá-lo (algoritmo U-CuRVE). Estes resultados foram apresentados no capítulo 2 .

2. A prova que o problema U-curve é NP-difícil; a demonstração deste teorema (teorema 3.2.3) foi feita no capítulo 3.

3. A demonstração de que o algoritmo U-CuRve possui um erro que torna-o um algoritmo de busca sub-ótimo na resolução do problema U-curve. Este resultado foi apresentado no capítulo 4.

4. Ainda no capítulo 4, a introdução de U-CURVE-SEARCH (UCS) uma versão corrigida e melhorada do algoritmo U-CURVE. UCS leva em consideração condições necessárias e suficientes para realizar podas no espaço de busca corrente sem risco de perder mínimos globais. Foi fornecida análise de complexidade de tempo do algoritmo UCS. 
5. A constatação que a busca de um início de caminho (i.e., um elemento do espaço de busca corrente) no algoritmo UCS é feita de maneira ineficiente. Formalizamos o problema da busca de início de caminho (problema 5.1.1) e também da variação do mesmo que exige a minimalidade (maximalidade) do elemento a ser encontrado. Conjecturamos a complexidade do problema da busca de início de caminho e provamos que a variação deste problema é NP-difícil. Estes resultados foram relatados no capítulo 5 .

6. Ainda no capítulo 5, a proposta de duas soluções alternativas para resolver o problema da busca de início de caminho. A primeira envolve a descrição do problema como um problema de Programação Linear Inteira e resolvendo-o diretamente utilizando um algoritmo do tipo branch-and-cut. Já a segunda faz uso da versão relaxada da variação do problema que exige minimalidade (maximalidade) para obter, via algoritmo Simplex, estimativas da região na qual deve ser buscado um início de caminho, para então, numa segunda etapa, realizar a busca propriamente dita, utilizando uma nova estrutura de dados e um esquema de enumeração.

7. Prova de corretude e análise de complexidade de tempo de U-Curve-Branch-And-Bound (UBB), um algoritmo de busca ótimo, do tipo branch-and-bound, para resolver o problema U-curve. Esta contribuição foi feita no capítulo 6.

8. Ainda no capítulo 6, a introdução do algoritmo Poset-Forest-SEARCH (PFS), um algoritmo de busca ótimo para resolver o problema U-curve. PFS generaliza o algoritmo UBB, no sentido que a busca é feita de forma bidirecional (i.e., as cadeias do reticulado Booleano são percorridas em ambas as direções). Foi fornecida análise de complexidade de tempo do algoritmo PFS.

9. A realização de experimentos ótimos e sub-ótimos, empregando instâncias reais e simuladas, e que avaliam o desempenho dos três algoritmos estudados neste trabalho. Foi fornecida uma análise de desempenho dos algoritmos, assim como uma verificação da consistência entre os resultados teóricos das análises de complexidade de tempo e os resultados obtidos nos experimentos. Estes resultados foram mostrados no capítulo 7.

10. Um relaxamento (generalização) do problema U-curve, com a definição do problema U-curve parcial (problema 8.1.1), no qual nem todas as cadeias do reticulado Booleano são decomponíveis em curvas em U. Foi demonstrado que o problema serve para modelar o problema de projeto de classificadores, assim como que os algoritmos UCS, UBB e PFS servem para resolver esta versão relaxada do problema U-curve. Estes avanços foram relatados no capítulo 8.

\subsubsection{Contribuições tecnológicas}

- O arcabouço featsel, desenvolvido para possibilitar os testes com os algoritmos introduzidos neste trabalho. Este arcabouço, desenvolvido em linguagem Orientada a Objetos, oferece classes e métodos para resolução de problemas de busca que lidam com o espaço de busca como um poset (e.g. um reticulado Booleano), assim como oferece flexibilidade na programação de novos algoritmos e de funções custo. Este arcabouço é descrito no apêndice A.

\subsection{Lista de publicações}

A partir dos resultados desta tese, o seguinte trabalho foi apresentado em congresso:

- Marcelo S. Reis, Carlos E. Ferreira e Junior Barrera

Estimation of Probabilistic Gene Networks using the UCS feature selection algorithm.

Proceedings of the 6th International Conference of the Brazilian Association for Bioinformatics and Computational Biology, 2010. p. SBN15.

Além disso, os seguintes artigos científicos foram preparados e enviados para publicação: 
1. Marcelo S. Reis, Carlos E. Ferreira e Junior Barrera

The U-curve optimization problem: theoretical advances, algorithm improvements and time complexity analysis [RFB12].

2. Marcelo S. Reis, Junior Barrera e Carlos E. Ferreira

An optimal branch-and-bound algorithm for the U-curve optimization problem: correctness, time complexity and experimental evaluation [RBF12].

3. Marcelo S. Reis e Junior Barrera

Solving problems in Mathematical Morphology through reductions to the U-curve problem [RB12].

\subsection{Possibilidades de trabalhos futuros}

Concluiremos esta tese com uma lista de algumas possibilidades de trabalhos futuros nesta linha de pesquisa:

- novos métodos e algoritmos para abordar o problema da busca de início de caminho. Uma possibilidade nesta área é a continuidade no desenvolvimento da solução que faz uso de uma enumeração do espaço de busca e que utiliza o método Simplex para delimitar as cardinalidades mínimas e máximas possíveis para um elemento qualquer do espaço de busca corrente;

- na área de melhorias do algoritmo UCS, o desenvolvimento de uma nova sub-rotina DFS, na qual são processados vários destes procedimentos ao mesmo tempo, o que poderia levar a uma exploração mais homogênea do espaço de busca e também aumentar a eficiência das podas. Ademais, esta abordagem pode ajudar a aliviar a necessidade da busca de início de caminho nas iterações do algoritmo;

- no relaxamento do problema U-curve, seria interessante a investigação das famílias de instâncias com oscilações para as quais os algoritmos UCS, UBB e PFS sempre encontram uma solução ótima. Outra possibilidade nesta área é a modelagem de espaços de busca restritos (i.e., posets definidos a partir de um reticulado Booleano) que possam ser entendidos como instâncias do problema U-curve parcial; estes espaços de busca são verificados no projeto de W-operadores crescentes [Hir00], assim como no de operadores multi-resolução [HJBBD02].

- desenvolvimento de uma versões paralelizadas dos algoritmos UCS, UBB e PFS. No caso específico do algoritmo UCS, existe a possibilidade de paralelização em duas etapas: na busca de um início de caminho (que não envolve o cálculo da função custo) e na busca em profundidade (a função custo é computada);

- utilizar os algoritmos para a resolução de problemas de interesse prático, através da redução das instâncias desses problemas para instâncias do problema U-curve. Uma possibilidade nesse sentido seria desenvolver uma redução para instâncias do U-curve a partir de instâncias do problema da equação de Minkowski na decomposição de elementos estruturantes [HB02]. Com isso, esse problema poderia ser resolvido empregando-se um dos algoritmos para o problema U-curve. 
CONCLUSÕES 


\section{Apêndice A}

\section{O arcabouço featsel}

Para possibilitar experimentos com os algoritmos apresentados neste trabalho, implementamos o arcabouço (framework) featsel [Rei12]. featsel foi desenvolvido seguindo o paradigma de Orientação a Objetos e implementado utilizando linguagem $\mathrm{C}++. \mathrm{O}$ arcabouço foi projetado levando-se em consideração as seguintes características:

- disponibilidade de classes e de métodos para manipulação do espaço de busca como um poset, com operações especiais para lidar com o caso especial do espaço de busca ser um reticulado Booleano;

- flexibilidade na programação de novos algoritmos de seleção de características e de funções custo, bastando criar uma nova classe derivada das classes abstratas Solver e CostFunction, respectivamente;

- possibilidade de implementação de algoritmos paralelizados, dentro do paradigma de compartilhamento de memória, utilizando para isso a API OpenMP [Boa10].

- possibilidade de implementação de algoritmos que fazem uso de técnicas de Programação Linear, utilizando para isso a biblioteca glpk [Mak11].

- manipulação de arquivos de entrada e de saída utilizando a linguagem de marcação XML. Os arquivos XML de entrada utilizam o Flex como analisador léxico [Pax10] e o Bison como analisador sintático [Pro10].

Todas as bibliotecas utilizadas são códigos abertos e livres e estão cobertas pela licença $G N U$ General Public License (GNU-GPL). Ademais, feat sel também está sob a licença GNU-GPL. Na figura A.1 exibimos o modelo de objetos do arcabouço feat sel, utilizando notação UML (Unified Modeling Language).

\section{A.1 Funções custo implementadas}

Para este trabalho, foram implementados, utilizando o arcabouço featsel, as seguintes funções custo:

- a redução polinomial de instâncias do problema da soma de subconjuntos para instâncias do problema U-curve (equação 3.1);

- a entropia condicional média penalizada (equação 7.2), empregando o algoritmo descrito no apêndice C. 


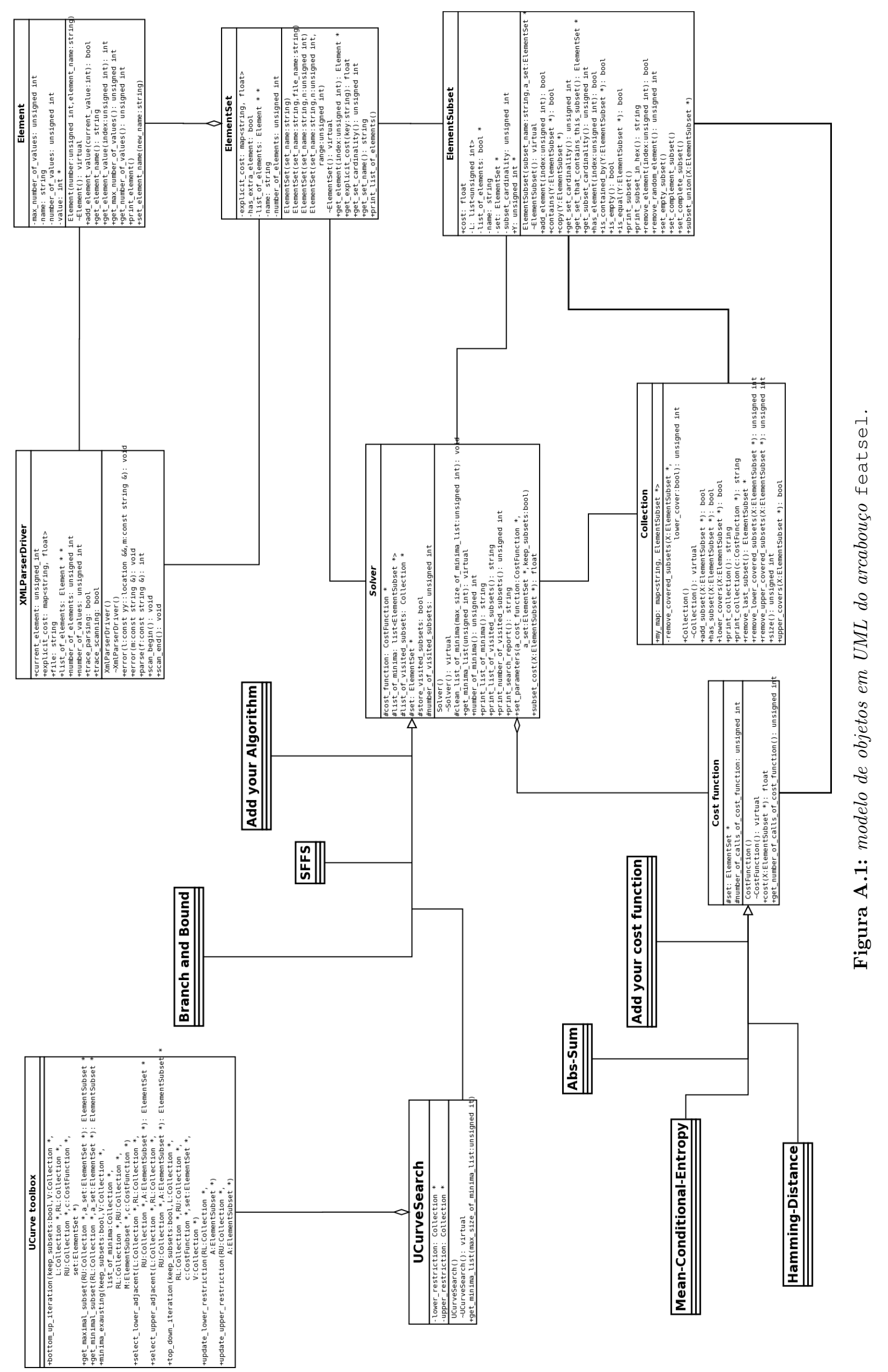




\section{A.2 Algoritmos implementados}

Também utilizando este arcabouço, foram implementados os seguintes algoritmos:

- UCS, apresentado no capítulo 4;

- UCS-2, apresentado no capítulo 5;

- UCS-3, apresentado no capítulo 5;

- UBB, apresentado no capítulo 6;

- PFS, apresentado no capítulo 6;

- ES, apresentado no apêndice B;

- SFFS, descrito em Pudil e colegas [PNK94].

Todos os resultados dos experimentos apresentados neste trabalho foram obtidos empregando estas implementações no arcabouço featsel. 
APÊNDICE A 


\section{Apêndice B}

\section{O algoritmo EXHAUSTIVE-SEARCH (ES)}

Para servir de base inicial de comparação dos algoritmos introduzidos neste trabalho, desenvolvemos Exhaustive-SEARCH (ES), um algoritmo de busca exaustiva.

ES foi implementado utilizando um contador binário para a enumeração dos $2^{n}$ elementos de um espaço de busca $\mathcal{P}(S)$. Este contador binário é baseado no algoritmo InCREMENT, descrito em Cormen et al. [CLRS01], página 408.

Descrição de algoritmo. ExhAustive-SEARch recebe um conjunto finito e não-vazio $S$ e uma função custo $c$ decomponível em curvas em $\mathrm{U}$, e devolve uma coleção $\mathcal{M} \subseteq \mathcal{P}(S)$ que contém todos os elementos em $\mathcal{P}(S)$ de custo mínimo.

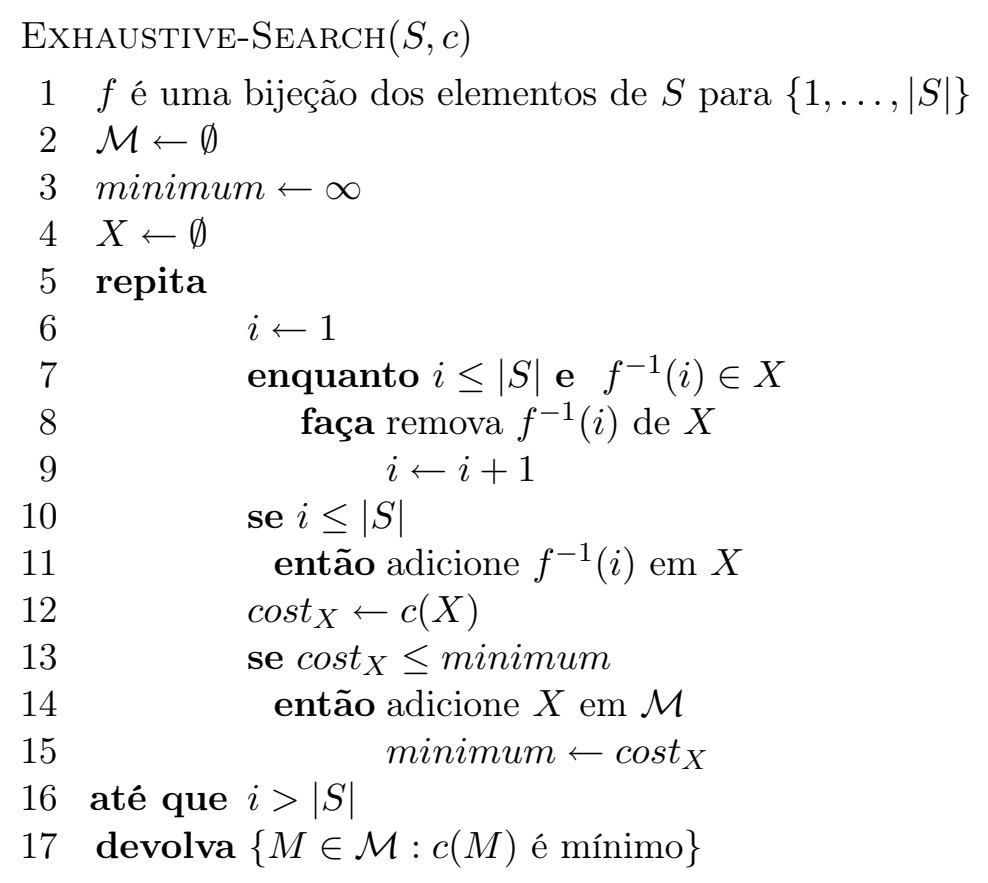

Análise de complexidade de tempo. ES gasta um tempo amortizado de $O(1)$ para visitar cada um dos $2^{n}$ elementos de $\mathcal{P}(S)$, uma vez que as operações do laço nas linhas 5-16 são equivalentes às operações executadas pelo algoritmo INCREMENT ([CLRS01], páginas 408-409), com exceção do código nas linhas 12-16. Cada vez que a função custo $c$ é computada (linha 12), o algoritmo consome $O(f(n))$ unidades de tempo; já a adição de $X$ na coleção $\mathcal{M}$ (linha 14$)$ consome $O(n)$ unidades de tempo. Portanto, o consumo de tempo total de ES é de

$$
O\left((1+n+f(n)) 2^{n}\right)=O\left((n+f(n)) 2^{n}\right)
$$

unidades de tempo. 
APÊNDICE B 


\section{Apêndice C}

\section{Implementação de função custo que utiliza entropia condicional média}

Esta seção apresenta uma análise de complexidade de tempo de um algoritmo que implementa a função custo da entropia condicional média com penalização (equação 7.2), descrita no capítulo 7.1.2. Este algoritmo foi empregado para realizar os experimentos com dados reais (i.e., projeto de W-operadores) que também foram apresentados no capítulo 7.

Sejam $S$ um conjunto não-vazio e $n$ a cardinalidade de $S$. Seja $\mathcal{D}$ uma árvore binária de busca, na qual cada elemento $\mathbf{A}$ tem três campos:

- "chave" é um elemento de $\mathcal{P}(X), X \subseteq S$. "chave" é utilizado para realizar uma busca em $\mathcal{D}$;

- "0" e "1" são dois inteiros, que representam o número de ocorrências de um dado elemento de $\mathcal{P}(X)$ com os rótulos 0 e 1 , respectivamente.

Suporemos que a árvore binária de busca $\mathcal{D}$ é implementada utilizando uma árvore binária balanceada de busca (e.g., árvores rubro-negras [GS78]). Dessa forma, a complexidade de tempo de uma busca é $O(n \log |\mathcal{D}|)$ unidades de tempo, de uma inserção é $O(n \log |\mathcal{D}|)$ unidades de tempo e de uma remoção é $O(n \log |\mathcal{D}|)$ unidades de tempo. Além disso, uma travessia (traversal) de $\mathcal{D}$ é feita em $O(|\mathcal{D}|)$ unidades de tempo.

Seja $t$ o número de amostras avaliadas. Definiremos agora uma tabela $T$ contendo as distribuições de pares $\langle y, \mathbf{x}\rangle$, no qual $y$ é um elemento de $\{0,1\}$ (o rótulo) e $\mathbf{x}$ é um elemento de $\mathcal{P}(X) \subseteq \mathcal{P}(S)$. $T$ tem no máximo $|\mathcal{P}(S)|=2^{n}$ linhas (i.e., ela tem no máximo todos os subconjuntos possíveis de $S$ ). Se $t<2^{n}$, então esta tabela tem $t$ elementos. Portanto, o número de linhas de $T$ é $l=\min \left\{t, 2^{n}\right\}$. Para cada linha $i$ de $T, 1 \leq i \leq l, T[i, k e y]$ é um elemento de $\mathcal{P}(S)$. Ademais, $T[i, j], j \in\{0,1\}$, é o número de vezes que $T[i, k e y]$ tem o rótulo $j$.

Inicialmente apresentaremos uma sub-rotina que calcula as distribuições dos elementos de $\mathcal{P}(X)$ que foram observados em $T$.

Descrição de algoritmo. CAlculate-Distributions recebe um elemento $X$ de $\mathcal{P}(S)$ e uma tabela $T$ contendo as distribuições dos elementos de $\mathcal{P}(S)$. Esta sub-rotina devolve uma árvore binária de busca $\mathcal{D}$, na qual cada elemento $\mathbf{A}$ contém a frequência que $\mathbf{A}[k e y]$ foi observado em $T$. Além disso, ela armazena em $\mathbf{A}[j], j \in\{0,1\}$, a frequência que $\mathbf{A}[k e y]$ tem o rótulo $j$. 


\section{Calculate-Distributions $(X, T)$}

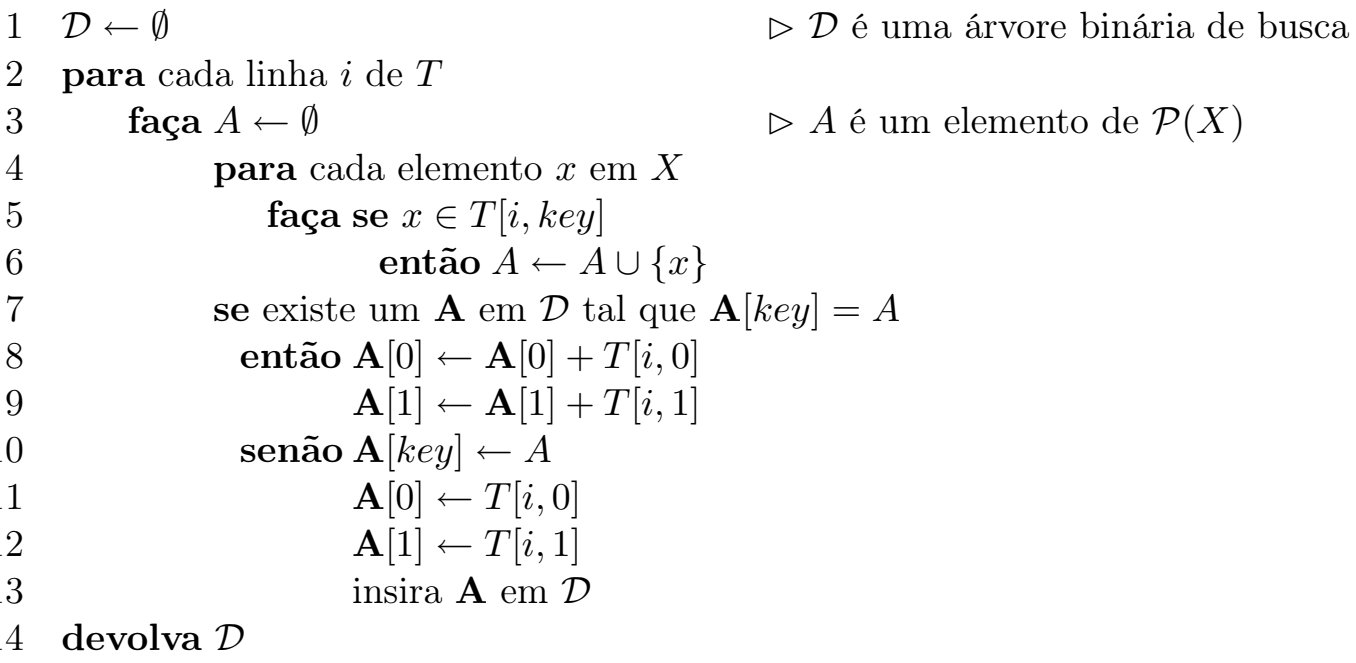

Análise de complexidade de tempo. O laço interno para nas linhas 4-6 itera no máximo $n$ vezes, exigindo a cada iteração $O(1)$. As operações de busca na linha 7 e de inserção na linha 13 requerem, cada, $O(n \log |\mathcal{D}|)=O(n \log l)$. Uma vez que o laço externo para nas linhas 2-13 itera $l$ vezes, Calculate-Distributions requer $O(n l \log l)$ unidades de tempo.

Apresentaremos agora o algoritmo principal.

Descrição de algoritmo. Mean-Conditional-Entropy recebe um elemento $X$ de $\mathcal{P}(S)$, uma tabela $T$ contendo as distribuições dos elementos de $\mathcal{P}(S)$,e o número de amostras $t$. Esta sub-rotina devolve a entropia condicional média penalizada de $X$.

\section{Calculate-Mean-Conditional-Entropy $(X, T, t)$}

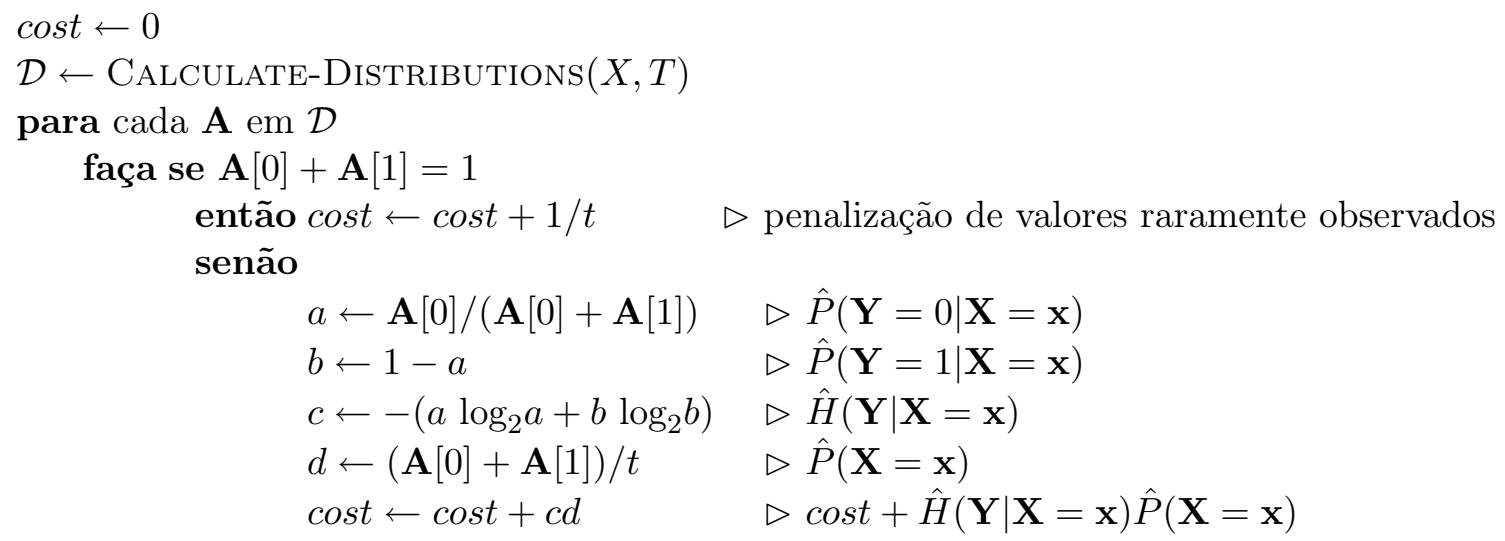

devolva cost

Análise de complexidade de tempo. O laço para nas linhas 3-11 é uma travessia de $\mathcal{D}$, a qual é feita em $O(|\mathcal{D}|)=O(l)$. Uma vez que a única chamada de Calculate-Distributions requer $O(n l \log l)$ unidades de tempo, o algoritmo Mean-Conditional-Entropy consome:

$$
O(l+n l \log l)=O(n l \log l)
$$

unidades de tempo. 


\section{Referências Bibliográficas}

[Bar12] J. Barrera. Núcleo como representação do espaço de busca corrente é computacionalmente intratável, 2012. Conversa pessoal. 65

[BB91] G.J.F. Banon e J. Barrera. Minimal representations for translation-invariant set mappings by Mathematical Morphology. SIAM Journal on Applied Mathematics, 51(6):1782-1798, 1991. 65

$\left[\right.$ BCJMJ ${ }^{+}$04] J. Barrera, R.M. Cesar-Jr, D.C. Martins-Jr, E.F. Merino, R.Z.N. Vêncio, F.G. Leonardi, M.M. Yamamoto, C.A.B. Pereira, e H.A. Del Portillo. A new annotation tool for malaria based on inference of probabilistic genetic networks. Em Proceedings of the 5th International Conference for the Critical Assessment of Microarray Data Analysis (CAMDA'04), páginas 36-40, 2004. 8

$\left[\right.$ BCJMJ $^{+}$07] J. Barrera, R.M. Cesar-Jr, D.C. Martins-Jr, R.Z.N. Vêncio, E.F. Merino, M.M. Yamamoto, F.G. Leonardi, C.A.B. Pereira, e H.A. Portillo. Constructing probabilistic genetic networks of Plasmodium falciparum from dynamical expression signals of the intraerythrocytic development cycle. Methods of Microarray Data Analysis V, páginas 11-26, 2007. 1, 6, 8

[Bir67] G. Birkhoff. Lattice Theory. American Mathematical Society, Providence, RI, 1967. 65

[Boa10] The OpenMP Architecture Review Board. The OpenMP API specification for parallel programming. http://openmp.org/wp, 2010. Último acesso em 20/7/2010. 127

[BS96] J. Barrera e G.P. Salas. Set operations on closed intervals and their applications to the automatic programming of morphological machines. Journal of Electronic Imaging, 5(3):335-352, 1996. 63

[BTHJH00] J. Barrera, R. Terada, R. Hirata-Jr., e N.S.T. Hirata. Automatic programming of morphological machines by PAC learning. Fundamenta Informaticae, páginas 229258, 2000. 97

[CLRS01] T.H. Cormen, C.E. Leiserson, R.L. Rivest, e C. Stein. Introduction to Algorithms. MIT Press, Cambridge, MA, USA, 2001. 16, 40, 131

[Dan98] G. Dantzig. Linear Programming and Extensions. Princeton University Press, 1998. 50,58

[DeG86] M.H. DeGroot. Probability and Statistics. Addison Wesley, Reading, MA, USA, 1986. 115

[DHS01] R.O. Duda, P.E. Hart, e D.G. Stork. Pattern Classification. John Wiley \& Sons, 2001. 1,6

[FW96] C.E. Ferreira e Y. Wakabayashi. Combinatória poliédrica e planos-de-corte faciais. X Escola de Computação, 1996. 50 
[GE03] I. Guyon e A. Elisseeff. An introduction to variable and feature selection. The Journal of Machine Learning Research, 3:1157-1182, 2003. 5

[GS78] L.J. Guibas e R. Sedgewick. A dichromatic framework for balanced trees. IEEE Annual Symposium on Foundations of Computer Science, 0:8-21, 1978. 87, 133

[Ham50] R.W. Hamming. Error detecting and error correcting codes. Bell System Technical Journal, 29(2):147-160, 1950. 113

[Has00] R.F. Hashimoto. Mudança de Estrutura de Representação de Operadores em Morfologia Matemática. Tese de Doutorado, Instituto de Matemática e Estatística, Universidade de São Paulo, Brasil, julho 2000. 115

[HB02] R.F. Hashimoto e J. Barrera. A note on Park and Chin's algorithm [structuring element decomposition]. IEEE Transactions on Pattern Analysis and Machine Intelligence, 24(1):139-144, 2002. 125

[Hei95] H.J.A.M. Heijmans. Mathematical Morphology: a modern approach in image processing based on algebra and geometry. SIAM review, 37(1):1-36, 1995. 65

[HHW88] F. Harary, J.P. Hayes, e H.J. Wu. A survey of the theory of hypercube graphs. Computers $\& 3$ Mathematics with Applications, 15(4):277-289, 1988. 11

[Hir00] N.S.T. Hirata. Projeto Automático de Operadores: Explorando Conhecimentos a Priori. Tese de Doutorado, Instituto de Matemática e Estatística, Universidade de São Paulo, Brasil, outubro 2000. 115, 119, 125

[HJBBD02] R. Hirata-Jr, M. Brun, J. Barrera, e E.R. Dougherty. Multiresolution design of aperture operators. Journal of Mathematical Imaging and Vision, 16(3):199-222, 2002. 120,125

$\left[\mathrm{JKP}^{+}\right.$94] G.H. John, R. Kohavi, K. Pfleger, et al. Irrelevant features and the subset selection problem. Em Proceedings of the eleventh international conference on machine learning, volume 129, páginas 121-129. San Francisco, 1994. 1, 5

[JM00] A.K. Jain e R.P.W.J. Mao. Statistical pattern recognition: A review. IEEE Transactions on pattern analysis and machine intelligence, 22(1):4-37, 2000. 1

[Joh02] D.S. Johnson. A theoretician's guide to the experimental analysis of algorithms. Data structures, near neighbor searches, and methodology: fifth and sixth dimacs implementation challenges, 59:215-250, 2002. 13

[JZ97] A. Jain e D. Zongker. Feature selection: Evaluation, application, and small sample performance. IEEE transactions on pattern analysis and machine intelligence, 19(2):153-158, 1997. xvii, 7, 8

[Kar72] R.M. Karp. Reducibility among combinatorial problems. Complexity of Computer Computations, páginas 85-103, 1972. 49

[Kit78] J. Kittler. Feature set search algorithms. Pattern recognition and signal processing, páginas 41-60, 1978. 7

[Lin91] J. Lin. Divergence measures based on the Shannon entropy. IEEE Transactions on Information Theory, 37(1):145-151, 1991. 6

[Mak11] A.O. Makhorin. GNU linear programming toolkit. http://www.gnu.org/software/ glpk, 2011. Último acesso em 20/7/2012. 52, 127 
[MG63] T. Marill e D. Green. On the effectiveness of receptors in recognition systems. IEEE transactions on Information Theory, 9(1):11-17, 1963. 7

[MJCJB04] D.C. Martins Jr, R.M. Cesar-Jr, e J. Barrera. W-operator window design by maximization of training data information. Em Computer Graphics and Image Processing, 2004. Proceedings. 17th Brazilian Symposium on, páginas 162-169. IEEE, 2004. 8

[MJCJB06] D.C. Martins-Jr, R.M. Cesar-Jr, e J. Barrera. W-operator window design by minimization of mean conditional entropy. Pattern Analysis $\&$ Applications, 9(2):139-153, 2006. 1, 6, 97, 98

[ML73] M. Michael e W.C. Lin. Experimental study of information measure and inter-intra class distance ratios on feature selection and orderings. IEEE Transactions on Systems, Man and Cybernetics, páginas 172-181, 1973. 7

[NC09] S. Nakariyakul e D.P. Casasent. An improvement on floating search algorithms for feature subset selection. Pattern Recognition, 42(9):1932-1940, 2009. 6, 7

[NF77] P.M. Narendra e K. Fukunaga. A branch and bound algorithm for feature subset selection. IEEE Transactions on Computers, 100(26):917-922, 1977. 6, 8, 67

[Pax10] V. Paxson. Fast lexical analyzer generator. http://flex.sourceforge.net, 2010. Último acesso em 20/7/2010. 127

[PNK94] P. Pudil, J. Novovicová, e J. Kittler. Floating search methods in feature selection. Pattern recognition letters, 15(11):1119-1125, 1994. 1, 6, 7, 8, 129

[Pro10] The GNU Project. Bison - gnu parser generator. http://www.gnu.org/software/bison, 2010. Último acesso em 20/7/2010. 127

[RB12] M.S. Reis e J. Barrera. Solving Mathematical Morphology problems through reductions to the U-curve problem. Enviado para publicação, 2012. 125

[RBF12] M.S. Reis, J. Barrera, e C.E. Ferreira. An optimal branch-and-bound algorithm for the U-curve optimization problem: correctness, time complexity and experimental evaluation. Enviado para publicação, 2012. 125

[RBMJ10] M. Ris, J. Barrera, e D.C. Martins-Jr. U-curve: A branch-and-bound optimization algorithm for U-shaped cost functions on Boolean lattices applied to the feature selection problem. Pattern Recogn., 43(3):557-568, 2010. 1, 6, 8, 9, 19, 67, 120

[Rei12] M.S. Reis. featsel - a flexible feature selection framework. http://www.ime.usp.br/ msreis/featsel, 2012. Último acesso em 20/7/2012. 12, 127

[RFB12] M.S. Reis, C.E. Ferreira, e J. Barrera. The U-curve optimization problem: theoretical advances, algorithm improvements and time complexity. Enviado para publicação, 2012. 125

[Rip08] B.D. Ripley. Pattern Recognition and Neural Networks. Cambridge university press, 2008. 6

[Ris08] M. Ris. Representação de sistemas biológicos a partir de sistemas dinâmicos: controle da transcrição a partir do estrógeno. Tese de Doutorado, Instituto de Matemática e Estatística, Universidade de São Paulo, Brasil, 2008. 1, 120

[Ros98] K. Rose. Deterministic annealing for clustering, compression, classification, regression, and related optimization problems. Proceedings of the IEEE, 86(11):2210-2239, 1998. 6 
[Sch00] A. Schrijver. A combinatorial algorithm minimizing submodular functions in strongly polynomial time. Journal of Combinatorial Theory, Series B, 80(2):346-355, 2000. 5

[SNP06] P. Somol, J. Novovičová, e P. Pudil. Flexible-hybrid sequential floating search in statistical feature selection. Structural, Syntactic, and Statistical Pattern Recognition, páginas $632-639,2006.7$

[SPK04] P. Somol, P. Pudil, e J. Kittler. Fast branch \& bound algorithms for optimal feature selection. IEEE Transactions on Pattern Analysis and Machine Intelligence, páginas 900-912, 2004. 1

[SPN $\left.{ }^{+} 99\right]$ P. Somol, P. Pudil, J. Novovicová, et al. Adaptive floating search methods in feature selection. Pattern recognition letters, 20(11-13):1157-1163, 1999. 6, 7

[SS89] W. Siedlecki e J. Sklansky. A note on genetic algorithms for large-scale feature selection. Pattern Recognition Letters, 10(5):335-347, 1989. 7

[SS93] W. Siedlecki e J. Sklansky. On automatic feature selection. Handbook of Pattern Recognition and Computer Vision, páginas 63-87, 1993. xvii, 1, 7, 10

[Ste76] S.D. Stearns. On selecting features for pattern classifiers. Em Proceedings of the 3rd International Joint Conference on Pattern Recognition, páginas 71-75, 1976. 7

[TK06] S. Theodoridis e K. Koutroumbas. Pattern Recognition. Elsevier, Academic Press, Amsterdam, New York, 2006. 1, 6

[VBHJ05] D.A. Vaquero, J. Barrera, e R. Hirata-Jr. A maximum-likelihood approach for multiresolution W-operator design. Em Computer Graphics and Image Processing, páginas 71-78. IEEE, 2005. 120

[Whi71] A.W. Whitney. A direct method of nonparametric measurement selection. IEEE Transactions on Computers, 20(9):1100-1103, 1971. 7 


\section{Índice Remissivo}

árvore, 11, 67

binária de busca, 133

balanceada, 87, 133

complementar, 76

rubro negra, 87, 133

algoritmo

\section{ASFFS, 7}

BEAM-SEARCH, 7

Exhaustive-Search, 12, 42, 93, 131

ISFFS, 7

INCREMENT, 131

Mean-Conditional-Entropy, 134

Minimum-Exhausting

erro, 19

Plus-L-Minus-R, 7

Poset-Forest-Search, 74, 97, 117, 121 princípios de funcionamento, 75 pseudo-código, 87

SBFS, 7

SBS, 7

SFFS, 1, 7, 13, 42, 97, 122

SFS, 7

U-Curve-Branch-AND-Bound, 67, 97, 117, 121

limitações, 74

U-Curve-Search, 40, 42, 50, 97, 117, 121

princípios de funcionamento, 20

pseudo-código, 29

U-Curve, 1, 9

erro, 19

UCS-2, 51

UCS-3, 59

HSFFS, 7

branch-and-bound, 8, 67

branch-and-cut, 51

branch-and-cut, 52

de busca, 6

de busca bidirecional, 10

de busca exaustiva, 8

descrição de, 11

genético, 7

paralelizado, 127 recozimento simulado, 7 seleção sequencial, 7

arcabouço, 12

diagrama do, 127

featsel, 12, 124, 127

aresta, 11, 22

busca

de início de caminho, 56

em profundidade, 39

cadeia, 15

maximal, 15

cladograma

algoritmos, 8

com U-CuRve, 9

classificador, 114

exemplo de aplicação, 117

núcleo, 115

cobertura de vértices

definição, 11

coleção de restrições, 9

aninhamento, 64

atualização, 29

estrutura de dados para, 53

inferiores, 9,29

superiores, 9, 29

conjunto

convexo, 65

mapeamento, 65

parcialmente ordenado, 15

potência, 5

curva em U, 1

exemplo, 1

decomposição, 67

de elementos estruturantes, 125

dual, 75

diagrama

de Hasse, 1, 19, 30, 55, 67, 74, 77

featsel, 127

UML, 127

distância

de Bhattacharyya, 6 
de divergência, 6

de Hamming, 19, 113

elemento

maximal, 30

minimal, 30

enumeração

método de, 55

erro absoluto médio, 115

esgotamento do mínimo, 9, 19

espaço de busca, 10

corrente, 30

estimação

de distribuições, 98

estrutura, 30, 87

campos, 30, 87

nó, 30, 87

experimento, 42

ótimo, 42, 51, 60, 93, 98

ambiente utilizado, 12

estrutura dos, 12

instâncias empregadas, 12, 97

sub-ótimo, 42, 93, 101

tipos de, 13

featsel, 12, 124, 127

floresta, 77

fluxograma

de leitura, 4

função custo, 5

da distância de Hamming, 113

complexidade, 12

decomponível em curvas em U, 15

distância de Bhattacharyya, 6

distância de divergência, 6

entropia condicional média, 6

penalizada, 98

Kullback-Leibler, 6

limitante de Chernoff, 6

MAE, 115

parcialmente decomponível em curvas em U, 113

submodular, 5

grafo, 11, 22, 30, 49

caminho, 11

extremo, 11

circuito, 11

cobertura de vértices, 11,49

conexo, 11, 31

desconexo, 11, 31

sub-grafo, 11, 67

tripartido, 65 trivial, 32

Hamming

distância, 19, 113

hipercubo, 11

imagem

amostras de, 98

binária, 98

intervalo, 11

interseções de, 62

mínimo

esgotado, 19

MAE, 115

Morfologia Matemática, 66

núcleo, 115

operador

crescente, 120, 125

multi-resolução, 120, 125

núcleo, 65

W-operador, 120, 125

ordenação

estável, 54

oscilações, 120, 125

poset, 15

problema

cobertura de vértices, 49

da equação de Minkowski, 125

da mochila, 17

início de caminho, 47

máximo, 48

mínimo, 47

seleção de características, 1, 5

soma de subconjuntos, 16, 97

U-curve, 15

complexidade, 15

U-curve parcial, 113

Programação Linear, 50

glpk, 127

Inteira, 50

pseudo-código

descrição de, 12

Rede Gênica Probabilística, 1

restrição, 11

inferior, 11

superior, 11

reticulado Booleano, 15

cintura, 48

extremidade, 48 
seleção de características

algoritmo de, 6

função custo de, 6

problema de, 1, 5

solução

viável, 50, 58

sub-rotina

BRANCH, 72

Calculate-Distributions, 133

DFS, 39, 61

Graph-Pruning, 37

LOWER-ELEMENT, 58

LOWER-ForEST-BRANCH, 87

LOWER-Forest-Pruning, 91

LOWER-PRUNing, 36

MAX-BRANCH-AND-CUT, 51

MAX-SimpleX, 58

MaXimal-Element, 30, 46, 50

Min-BranCH-AND-CUT, 51

Min-Simplex, 58

Minimal-Element, 30, 46, 50

Minimum-Exhausting, 19

erro, 19

Remove-Graph, 38

Search-Lower-Children, 89

SEArch-Lower-Root, 90

SeArCh-Upper-Children, 89

SeArch-Upper-Root, 90

Select-UnVisited-Adjacent, 35

UPDATE-LOWER-RESTRICTION, 29

UPDATE-UPPER-RESTRICTION, 29

UPPER-ELEMENT, 59

UPPER-FOREST-BRANCH, 88

UPPER-Forest-Pruning, 90

UPPER-PRUNING, 37

topologia

de grafo, 32

de percorrimento, 9

U-curve

parcial, 113

problema, 67

de decisão, 15

UML, 127

vértice, 11, 22

vetor característico, 10, 50, 58, 64

W-operador, 1, 12, 97

XML, 127 
ÍNDICE REMISSIVO 